\title{
Estimating Gulf of Maine Zooplankton Distributions Using Multiple Frequency Acoustic, Video and Environmental Data
}

by

Joseph David Warren

B.S., Harvey Mudd College, 1994

Submitted to the

Department of Ocean Engineering, MIT

and the

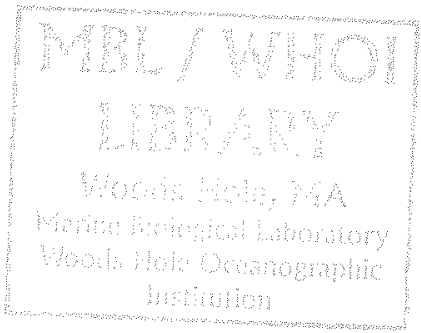

Department of Applied Ocean Physics and Engineering, WHOI

in partial fulfillment of the requirements for the degree of

DOCTOR OF PHILOSOPHY

at the

MASSACHUSETTS INSTITUTE OF TECHNOLOGY

and the

WOODS HOLE OCEANOGRAPHIC INSTITUTION

February 2001

(C)2000 Joseph D. Warren. All rights reserved.

The author hereby grants to MIT and WHOI permission to reproduce and distribute publicly paper and electronic copies of this thesis document in whole or in part. 


\title{
Estimating Gulf of Maine Zooplankton Distributions Using Multiple Frequency Acoustic, Video and Environmental Data
}

\author{
by \\ Joseph David Warren \\ Submitted to the \\ Department of Ocean Engineering, MIT \\ and the \\ Department of Applied Ocean Physics and Engineering, WHOI \\ on September 29, 2000, in partial fulfillment of the \\ requirements for the degree of \\ DOCTOR OF PHILOSOPHY
}

\begin{abstract}
This thesis develops methods useful for estimating zooplankton distributions in the field by combining acoustic scattering models and an integrated set of field data. The accuracy of existing scattering models for fluid-like and elastic-shelled animals is determined by analysis of scattering data from individual animals in a laboratory tank. Results indicate that simple two-ray scattering models are accurate and allow predictions of size or orientation of an animal to be made for certain animal orientations. A scattering model for gas-bearing zooplankton is compared with in situ multiple frequency acoustic measurements from siphonophores. Estimates of the numerical density of these animals are made using echo integration data from a scientific echo-sounder. Multiple frequency acoustic scattering data from a survey of an internal wave are analyzed to determine the contributions from biological and physical sources. Net tow data provide information about biological scatterers while temperature and salinity profiles are used with a theoretical scattering model to predict contributions from physical sources. Results indicate that scattering from physical sources is comparable to that from biological sources in certain regions and that scattering spectra may be used to distinguish these sources. Improved estimates of biomass from acoustic scattering data were made by accounting for the scattering contributions from physical sources. This is the first work to quantify the scattering contributions from biological and physical sources of scattering in a field study.
\end{abstract}

Thesis Supervisor: Dr. Timothy K. Stanton

Title: Senior Scientist, WHOI

Thesis Supervisor: Dr. Peter H. Wiebe

Title: Senior Scientist, WHOI 
It is better to fail in originality than to succed in imitation. He who has never failed somewhere, that man cannot be great. If it be said that continual success is proof that a man wisely knows his powers, it is only to be added that, in that case, he knows them to be small.

- Herman Melville

Don't believe the hype.

- Public Enemy

A career? Ive thought about this quite a bit sir and I would have to say considering what's waiting out there for me, I don't want to sell anything, buy anything or process anything as a career. I don't want to sell anything bought or processed or buy anything sold or processed or repair anything sold, bought or processed as a career. I don't want to do that.

- Lloyd Dobler

He never was a silly little boy

Who whispered in the class or threw spit balls,

Or pulled the hair of silly little girls,

Or disobeyed in any way the laws

That made the school a place of decent order

Where books were read and sums were proven true

And paper maps that showed the land and water

Were held up as the real wide world to you.

Always, he kept his eyes upon his books:

And now he has grown to be a man

He is surprised that everywhere he looks

Life rolls in waves he cannot understand,

And all the human world is vast and strange -.

And quite beyond his Ph.D.'s small range.

- Langston Hughes 


\section{Acknowledgments}

This thesis is dedicated to my grandparents: Olive and Mel Bontrager, and Bette, Bruce and Nettie Warren. All of whom thought, I was much smarter than I really am. My parents and sister can finally stop asking me when I'm going to finish, l'm done. Thanks for your support and love.

My advisors, Tim and Peter, gave me a wonderful opportunity to work (and learn) alongside two excellent scientists, and their commitment and passion toward science are inspiring. I can't thank them enough. Jim Lynch was my first advisor and introduced me to oceanography, in addition to serving on my committee. While he may now regret it, he's responsible for my being in this field. Professor Nicholas Makris taught me more in one hour of my Part II exam then I learned in several courses. I appreciate the effort and time he spent serving on my committee. Mark Benfeld was insightful, useful, and the only fellow zymurgist on my committee. Through multiple cruises, workshops, and beers; his advice has been most appreciated. Discussions with Harvey Seim and Ray Schmitt were very helpful (and necessary) in the writing of Chapter Four. Clarence Clay, Marek Moszyński, and Malinda Sutor provided advice regarding Chapter Three. Andone Lavery graciously read and commented on several chapters of this thesis. George Frisk is a fellow jazz fan and I'm thankful to him for chairing my defense.

There are numerous people who have made this experience worthwhile and also a lot of fun. I'm privileged to have them as friends. Steve Jayne and Lihini Aluwihare were the first people I met here in Woods Hole. Lihini was a bundle of energy and fun and, most importantly, helped remind us how important disco is in our daily lives. As for Steve, I can't think of any better way to describe his friendship than the fact that he helped me wash my hair when I was in my halo brace, I owe him big time for that. Sean McKenna, Kyle Becker, Ben Evans and Chris Linder were all gluttons for the Course 13 treatment. Hours and hours of problem sets, test studying, qualifying exam complaining, and even more time spent shooting the breeze and drinking beer made my years at MIT somewhat bearable. Ryan Frazier came out here from HMC with me, and even lived with me for a year before he couldn't stand it anymore. The fact that he still talks to me after knowing me for more than 10 years is an amazing feat.

The original residents of 24 Millfeld (Bill, Jon, Kira, Peter, Danny, Kitty, Patches, Zorro): we roasted pigs, baked clams, paddled kayaks, sailed boats, snowshoed New Hampshire, made dinner, and had the most amount of fun humanly possible. The residents of D-Entry at MacGregor Hall never ran away when I wandered around in my dog slippers and cage. Not many people will take cookies baked by Frankenstein, but they did.

My fellow JP classmates are an exceptional group of people, not to mention they're all wicked smart. From the initial pot-lucks to the defense parties, it's been a fantastic group of people to have as my peers. Ann P. was always up for a "date". Liz K. survived 10 hours in a car with me and didn't have to be institutionalized. Sheri W. never tackled me too hard while playing rugby. Brenda J. attended Lollapalooza 
with me. Nicole P. survived living with me during our thesis writing. Mike A. reminded me you'te never too old to have fun. Tom M., Lou StL., and Mike B. were the original members of Vomelette. Suzie W.'s enthusiasm for school was a complete contrast to mine. Fellow NRQ members, Albert and Mak, made the last 6 months of grad school very survivable. Doc Reddy made the best brunches l've ever eaten, taught me many acronyms, was a co-founder of PNF and rescued me after my kayak was attacked by a rogue buoy. He's a great friend.

Rich Pawlowicz and Art Newhall taught me most of what I know about MATLAB and computers and were always willing to help me out and give me advice. Mari Butler and Nancy Copley taught me everything I know about plankton counting, sorting; and identifying. Dezhang Chu was always helpful (and more importantly knowledgeable) about any acoustics, electronics or computer question I had. Heidi Sosik, Speck, Ru, and Anne were ideal shipmates. Linda Martin Traykovski gave me excellent advice on how to handle the advising of Peter and Tim. Chuck Greene allowed me to spend summers in Santa Cruz and New Hampshire and was a great watch-mate on the "BIOMAPPER" cruises. Kouichi Sawada invited me to Japan and was a perfect host.

Everybody in the Education Office has been very helpful and supportive, and Julia W. never kicked me out of her office when I needed to vent. Id like to thank the Ocean Engineering department at, MTT for proving that a major spinal cord trauma isn't the worst thing that can happen to you in grad school. The nurses and Doctors at Massachusetts General Hospital are superb. If you're ever going to get hurt, go there.

Many other members of the WHOI population deserve special mention here for making my grad school career interesting and entertaining: Jim P., Steve F., Ben R., Andy G., Stace B., Karen F., Jason G., Tad S., Dan M., Chris R., Oscar P., Charlie S., Brian S., Dana S. (and Will). Muchas Gracias. The AOPE softball team, the beach volleyball gang, and the MITRFC offered some fine reasons to avoid work.

Finally, the American taxpayers generously supported my graduate school career via: Office of Naval Research grants N00014-95-1-0287 and N00014-96-1-0878; NOAA's National Undersea Research Program grants NAGL-98-01A and NAGL-98-01B; an ONR Graduate Traineeship Award (N00014-00-1-0052); a Paul M. Fye Teaching Fellowship; and the WHOI Education Office. 


\section{Contents}

1 Introduction 11

1.1 Motivation ....................... 11

1.2 Thesis Description . . . . . . . . . . . . . . . . 13

1.3 Oceanography of the Gulf of Maine and Georges Bank . . . . . . . . 14

1.3 .1 Biology . . . . . . . . . . . . . . . . 16

1.4 Bioacoustics ......................... . . . 19

1.4.1 Historical Perspective . . . . . . . . . . . . . . . . . . . . . . . 19

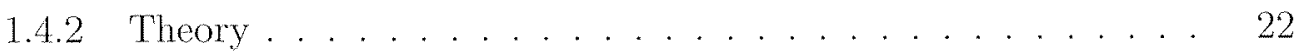

1.4 .3 Scattering Models . . . . . . . . . . . . . . 25

1.5 BIOMAPER-II . . . . . . . . . . . . . . . 26

2 Effect of Animal Orientation on Acoustic Estimates of Zooplankton $\begin{array}{ll}\text { Properties } & 37\end{array}$

2.1 Introduction . . . . . . . . . . . . . . . . . . . . . . . . . 38

2.2 Theory . . . . . . . . . . . . . . . . . . . . . . 39

2.2 .1 Fluid-like Animals . . . . . . . . . . . . . . 40

2.2 .2 Elastic-shelled Animals . . . . . . . . . . . . . . . . . . 42

2.2 .3 Pulse Compression Processing . . . . . . . . . . . . . 44

2.3 Experimental Methods . . . . . . . . . . . . . . . 46

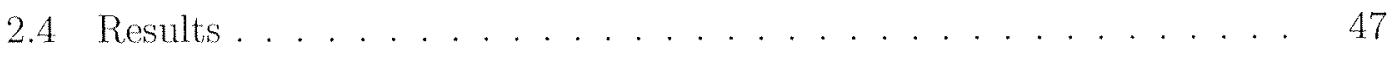

2.4 .1 Fluid-like Animal . . . . . . . . . . . . . . 47

2.4 .2 Elastic-shelled Animal . . . . . . . . . . . . 55 
3 In Situ Measurements of Acoustic Target Strengths of Siphonophores, $\begin{array}{ll}\text { a Gas-bearing Zooplankter } & 63\end{array}$

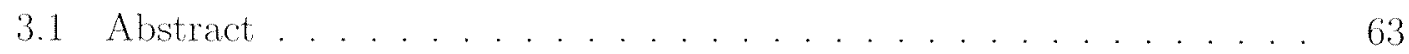

3.2 Introduction . . . . . . . . . . . . . . . . 64

3.3 Methods . . . . . . . . . . . . . . . . . . . . . 68

3.3 .1 ROV System . . . . . . . . . . . . . . . . . 68

3.3.2 ROV Data Collection . . . . . . . . . . . . . 70

3.3.3 ROV Calibration and Data Processing . . . . . . . . . 71

3.3.4 Towed Echosounder Surveys . . . . . . . . . . . . . 72

3.3 .5 Net Tows . . . . . . . . . . . . . . . . . . 72

3.3.6 Acoustic Scattering Model . . . . . . . . . . . . . . 73

3.4 Results. . . . . . . . . . . . . . . . . . . . . . 74

3.5 Discussion . . . . . . . . . . . . . . . . . . . . . . . . 79

4 Biological and Physical Sources of Scattering in an Internal Wave 85

4.1 Introduction . . . . . . . . . . . . . . . . . 85

4.2 Methods . . . . . . . . . . . . . . . . . . . . . . . . . . 89

4.2.1 Acoustics . . . . . . . . . . . . . . . . . 99 90

4.2 .2 Zooplankton . . . . . . . . . . . . . . . . 94

4.2 .3 Microstructure . . . . . . . . . . . . . . . . . . 98

4.3 Results. . . . . . . . . . . . . . . . . . . . . 104

4.3.1 Acoustic, Environmental and Net Tow Data for 16 October 1997105

4.3.2 Sources of Acoustic Scattering . . . . . . . . . . . . 136

4.3.3 Inversion of Multiple Frequency Acoustic Data for Biological and Physical Parameters . . . . . . . . . . . . . . . . . 142

4.4 Discussion . . . . . . . . . . . . . . . . . . . . . . . . . . . 146

4.4.1 Distinguishing Between Different Sources of Scattering: Zooplankton and Microstructure . . . . . . . . . . . 146 
4.4.2 Contributions of Microstructure to Measured Scattering . . . . 147

4.4 .3 Inversion of Acoustic Data for Estimates of $\epsilon$. . . . . . . . . 148

4.4.4 Implications for Single and Multiple Frequency Acoustic Surveys 150

4.4 .5 The Inverse Problem . . . . . . . . . . . . . . . . . . . . 151

5 Conclusions 155

5.1 Zooplankton Scattering Models . . . . . . . . . . . . 156

5.1 .1 Effect of Animal Behavior . . . . . . . . . . . . . 156

5.1 .2 In Situ Verification of Models . . . . . . . . . . . . . . . 157

5.2 Accuracy of Forward Problem Calculations . . . . . . . . . . . . 158

5.3 Contributions of the Thesis . . . . . . . . . . . . 160

A Analysis of Biological and Physical Sources of Scattering from a $\begin{array}{ll}\text { Nearby Region } & 163\end{array}$

A.1 Acoustic, Environmental, and Net Tow Data for a Nearby Region . . 163

A.1.1 Acoustic Scattering Data . . . . . . . . . . . . 163

A.1.2 MOCNESS \#7 . . . . . . . . . . . . . . . . . . 169

A.1.3 Water Column Hydrography . . . . . . . . . . . . . . . 173

A.1.4 Forward Problem Calculations . . . . . . . . . . . . . . 173

A.1.5 Prediction of $\epsilon$ Using Thorpe lengths . . . . . . . . . 178

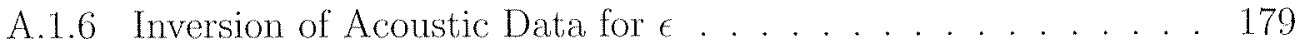

A.1.7 Modified Forward Problem calculations . . . . . . . . . . . . 184

A.1.8 Sources of Biomass and Acoustic Scattering . . . . . . . 187

B Qualitative Analysis of VPR and Acoustic Scattering Data from the $\begin{array}{ll}\text { Internal Wave Survey } & 197\end{array}$

B.1 Transect One - Above the Internal Wave . . . . . . . . . . . . . . . 197

B.2 Transect Two - Below the Internal Wave . . . . . . . . . . . . . . . 200

B.3 Transect Three - In the Midst of the Internal Wave . . . . . . . . . 200 
C Zooplankton Scattering Models 205

C.1 Variables ......................... . . . 205

C.2 Fluid-like Animals . . . . . . . . . . . . . . . . 206

C.3 Copepods ........................... 206

C.4 Elastic-shelled Animals . . . . . . . . . . . . . . . . . . 208

C.5 Gelatinous Animals . . . . . . . . . . . . . . . . . . . . . . . . . 208

C.6 Siphonophore Pneumatophore . . . . . . . . . . . . . . . . . . . 208 


\title{
Chapter 1
}

\section{Introduction}

\author{
I do not know what I may appear to the world; but \\ to myself I seem to have been only like a boy playing \\ on the sea-shore, and diverting myself in now and \\ then finding a smoother pebble or a prettier shell \\ than ordinary, whilst the great ocean of truth lay all \\ undiscovered before me.
}

Isace Newton

\subsection{Motivation}

Over the last two decades, the once thriving fishing industry in New England has become decimated (Chamberlain, 1985; Dumanoski, 1988; Terry, 1994). Largely due to over-fishing, the stocks of cod and haddock located on Georges Bank collapsed. This caused a drastic impact not only on the marine ecosystem, but also on the economy of the New England area (Editorial, 1995; Zitner, 1999). The United States government spent a large sum of money on "emergency management" of the fishing industry and also began to fund more studies of the fishing stocks and fishing practices on Georges Bank.

Beginning in the late 1990's, some of the fishing stocks began to show signs of recovery, while other species and regions are still struggling (Allen, 1998; Laidler, 1999; Howe, 1999; Allen, 1999). Fisheries management agencies are in the difficult position of trying to balance the needs of the fishermen for economic subsistence and the 
long-term health of the Georges Bank fishery. Fortuitously, this region has also been the subject of many studies (including modeling, long time-series measurements, and novel instrumentation methods) by scientists throughout the world. In fact, Georges Bank is the subject of the U.S. GLOBEC (GLOBal ocean ECosystems dynamics) Northwest Atlantic program, a multi-year, inter-disciplinary study aimed at understanding the population dynamics of the cod and haddock. This economical and ecological crisis has provided a unique opportunity for fisheries management agencies to obtain a wealth of data and knowledge about the ecosystem they are trying to regulate.

Because of the importance of assessing the fish stocks of the Georges Bank region, there has been a large effort (including this thesis) directed towards the study of the zooplankton of this region. This area of study includes not only Georges Bank, but also the Gulf of Maine, which is a source for many of the zooplankton that end up on Georges Bank (Figure 1-2). The zooplankton are the primary food source for the early life history stages of the commercially important fish stocks, such as cod, haddock, and other ground fish. It is vital that the distribution, taxonomic composition, and abundance of zooplankton populations are understood in order to properly determine their effect on the fish population. Without knowledge about the zooplankton, it is difficult to determine whether low fish stocks were the result of a dearth of food sources or human impact through over-fishing.

There are other mammals besides humans that are concerned with the distribution of zooplankton in the Gulf of Maine and Georges Bank. They use acoustic techniques to locate and assess these populations (as did the research in this thesis) and depend on them for survival. Right, humpback, fin and minke whales (Eubalaena glacialis, Megaptera novaeangliae, Balaenoptera physalus, and Balaenoptera acutorostrata respectively) are commonly found in the waters around Cape Cod and the Gulf of Maine. Unfortunately there are approximately only 300 Northern Right Whales left in the world and their population is currently declining (Caswell et al., 1999). These endangered species generally feed on copepods (predominantly Calanus sp.) which 
are one of the main species of interest in this thesis. Proper assessment of copepod populations will assist marine mammal scientists in determining the role of food availability in their population ecology.

\subsection{Thesis Description}

This chapter provides an introduction to the following topics: the general oceanography of the Gulf of Maine and Georges Bank; the use of acoustics to study zooplankton distributions; and a description of the instrument used in this work, BIOMAPER-II.

Chapter Two analyzes and validates simple two-ray scattering models for describing the scattering from two distinct types of zooplankton. These scattering models and the use of pulse compression signal processing techniques allow information to be obtained from a single insonification of an animal. This information can be used to estimate the size of an individual animal quite accurately. The effect of animal orientation on the acoustic target strength is also discussed.

Chapter Three is a first-of-a-kind study of the in situ scattering properties of siphonophores. These pelagic, gelatinous zooplankton were studied using a multiple frequency acoustic array mounted on a Remotely Operated Vehicle. The vehicle was used to track individual animals. Scattering strengths for individual animals were found. These results combined with data collected from a scientific echosounder were used to estimate the numerical density of siphonophores in the study region.

Chapter Four analyzes a set of BIOMAPER data from the Gulf of Maine, where an internal wave was studied. By examining the differences in scattering strength at the different frequencies, areas of the internal wave packet can be determined to be caused by zooplankton or other scattering processes. A theoretical model for scattering from temperature and salinity microstructure is included in a "Forward Problem" analysis of these regions. The Forward Problem takes net tow information and using the abundance and taxonomic information, calculates how much scattering (using theoretical scattering models) the animals and other scattering processes in the water column would cause. This study is the first to quantitatively measure the 
contributions of both biological and physical sources of acoustic scattering in a field study.

Chapter Five summarizes the contributions of this thesis and discusses topics for future work in this field. There are also three appendices included: analysis of BIOMAPER-II data from 14 October 1997 where an internal wave is clearly seen during MOCNESS tow \# 7, presentation of the data from the Video Plankton Recorder during the three transects through the internal wave on 16 Oct 1997, and a summary of the acoustic scattering models used for the different zooplankton taxa.

This thesis discusses various zooplankton scattering models and how they are implemented in analyzing acoustic scattering data for biological information. Acoustic techniques provide a useful tool for biologists to study zooplankton populations, however understanding scattering models and their limitations is vital for analysis of field data. The goal of using acoustic techniques is to accurately estimate information about zooplankton populations. In order for this to occur, all of the scattering processes in the ocean (including non-biological ones) must be understood.

\subsection{Oceanography of the Gulf of Maine and Georges Bank}

\section{Historical Perspective}

The first study of the plankton, fishes and physical oceanography of the Gulf of Maine and Georges Bank was undertaken by Henry Bryant Bigelow (Figure 1-1). Although commercial fishing of this region had been underway since the mid-eighteenth century, Bigelow was the first to scientifically survey the waters. His plankton work consisted of many cruises from 1912 to 1920 whose results are summarized in Bigelow (1926). Other studies conducted by him have described the fishes (Bigelow and Schroeder, 1953) and physical oceanography of the region (Bigelow, 1927).

Bigelow stated that a "mare incognitum" lay before him in his work, however his conclusions and data have been remarkably accurate (given his technological limitations). The one drawback of his work was that his results are for the most part 


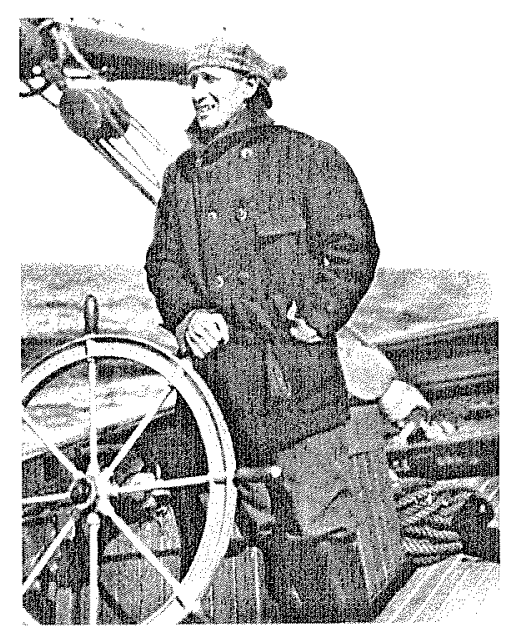

Figure 1-1: Henry Bryant Bigelow sailing the USFC schooner Grampus in 1912. (Courtesy of the Northeast Fisheries Science Center Photo Archives)

qualitative. From over one thousand zooplankton tows in the Gulf of Maine, he stated:

... calanoid copepods are predominant members at all seasons, except where deposed ... by temporary swarming of some other or usually larger animal.

For the most part, this statement still holds true today. The six most abundant (number of animals / volume) species on Georges Bank are all copepods (Davis, 1987). With advances in technology, scientists have begun to quantify many of Bigelow's observations. A summary of this work (Davis, 1987) has concluded that:

The available evidence indicates that zooplankton species composition, distribution and abundance are substantially the same as they were when Bigelow took his samples.

\section{Physical Processes}

In order to understand the zooplankton behavior, abundance and distribution of this region, one must also understand the physical forces that control the plankton populations in this area. A review of the Georges Bank ecosystem (Backus, 1987) 
summarizes the current state of knowledge of the Bank and synthesizes a substantial amount of information about the waters of the Gulf of Maine.

The Gulf of Maine is a semi-enclosed sea formed by an indentation in the continental shelf located between Cape Cod, United States and Nova Scotia, Canada (Figure 1-2). Land masses form barriers on the northern, western and eastern edges of the Gulf and there are numerous river inputs along this boundary. The southern side of the Gulf of Maine is flanked by a large $(300 \mathrm{~km}$ by $150 \mathrm{~km}$ ), shallow (40 m water depth at the crest) topographical feature named Georges Bank.

An interesting circulation feature of this region is that two opposing gyres are formed in the Gulf of Maine basin and around Georges Bank. Driven by density differences and tidal currents, a cyclonic gyre forms within the Gulf of Maine while an anti-cyclonic gyre circulates around Georges Bank. These two circulations are vitally important to the zooplankton life cycle, because they transport larval zooplankton to, and entrain them in regions with high abundances of food. Strong tidal currents (up to $1 \mathrm{~m} / \mathrm{s}$ ) are quite frequent in this region due to the Gulf of Maine having only two narrow passages to the Northwest Atlantic ocean (at the Great South Channel and the Northeast Channel).

In addition to the tidal forcing, seasonal storms have a large impact on the structure of the water column (most prominently on the top of Georges Bank). The gyre circulations intensify during the spring and summer seasons, while during winter (when large Nor'easter storms are frequent), the flow is typically offshore due to wind forcing. Additionally these storms ensure that the upper water column (approximately $50 \mathrm{~m}$ deep) around the Bank is vertically well-mixed.

\subsubsection{Biology}

Although there are msubstantiated reports that in the mid-1800's a section of Georges

Bank was exposed at low water and used as a field for a baseball game played by a ship's crew, it is better known as one of the largest fishing grounds in New England. In order to support a successful fishery, there needs to be an abundant supply of food 


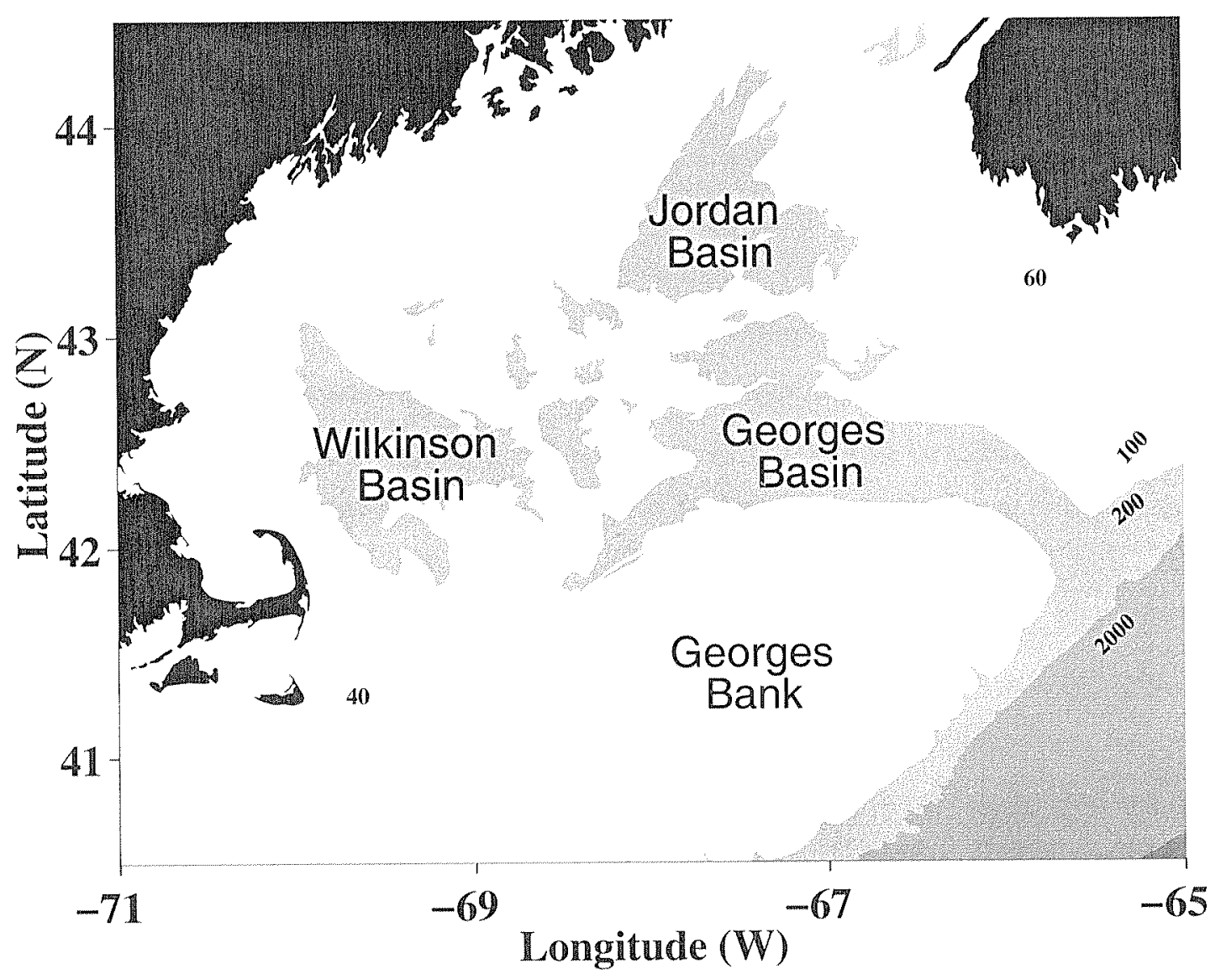

Figure 1-2: Bathymetry contours (in meters) of Georges Bank and the Gulf of Maine. The three deep basins in the Gulf of Maine were the focus of five BIOMAPER-II cruises from 1997-1999. 
for the fish in their early life stages. Zooplankton, predominantly copepods, fill this need for the Gulf of Maine and Georges Bank. While the life histories of the dominant copepods vary, the pattern displayed by Calanus finmarchicus illustrates the linkages between the Gulf of Maine and Georges Bank. A simplified C. finmarchicus life cycle is that from December thru February female copepods leave the deep basins of the Gulf of Maine, where they have spent the warm months in diapause. The Gulf of Maine circulation carries them to the western edge of Georges Bank where its circulation pattern then transports them clockwise around the Bank. Due to the winter storms, the Bank's waters are well-mixed, nutrient-rich, and feature a spring diatom bloom which the copepods arrive in time to feed on. Copepod biomass peaks in the summer months, with a sharp decrease in early fall due to food limitation and predation by gelatinous zooplankton, however temporal and spatial variability play an important role in determining where and when zooplankton species will be found.

Other species commonly found in the Gulf of Maine and also important to this thesis include: euphausiids, decapod shrimp, pelagic pteropods, and gelatinous zooplankton. Euphausiids and decapod shrimp can be quite abundant in the deep basins of the Gulf of Maine. Pelagic thecosomate pteropods such as Limacina retroversa are less commonly found in these waters, however they are very strong acoustic scatterers and can occur in quite dense patches. Gelatinous zooplankton, such as salps and siphonophores, are among the least understood animals in the Gulf of Maine waters. In the case of the latter, due to their composition, net tows are often unable to capture pristine (or even whole) specimens, therefore their distribution and abundance are poorly understood. However, siphonophores may be a major predator of copepods and other smaller zooplankton, and thus are important to study to understand their role in the zooplankton ecosystem. Additionally, siphonophores in the Gulf of Maine have on occasion clogged fishing trawl nets, thereby resulting in economic losses for fishermen (Rogers et al., 1978). 


\subsection{Bioacoustics}

\subsubsection{Historical Perspective}

Due to the rapid absorption of light by sea water, sound has become the preferred tool of oceanographers to study many oceanographic processes. Although sound is also absorbed by sea water, under certain conditions certain frequencies of sound can travel around the world and still be detected (Baggeroer et al., 1994). The development of acoustic technology increased rapidly after the sinking of the Titanic and resulted in numerous boats being outfitted with echo-sounding (or iceberg-detecting) devices. The military needs of World War II provided ocean acoustics with a strong influx of both funding and skilled scientists. Just after the war, many papers were published commenting on the existence of the Deep Scattering Layer (DSL) (see references in Smith (1954)). Early studies (Dietz (1948) and Johnson (1948)) were astonishingly accurate in their predictions of the cause of this scattering phenomenon. Biological sources were thought to be the source of the DSL due to the migration of the layer during the day. Dietz (1948) speculated on the possibility of small bubbles in these animals being the reason for the strong scattering. [These ideas were not universally accepted at the time. In the margin of the Marine Biological Laboratory and Woods Hole Oceanographic Institution Library copy of this journal article, a reader has written the comment "Idiot!".]

Further studies (Marshall (1951) and later Hersey and Backus (1954)) reported that pelagic fishes were the likely cause of the DSL. In the next decade, numerous studies determined that the gas-bladder of these pelagic fish was the dominant scattering mechanism and that the movement of the layer was caused by the diel migration of the fish.

In the mid-1960s, Barham $(1963,1966)$ explored the DSL with a submersible and observed that other animals besides fish were present. Gas-bearing siphonophores were abundant and contributed to the acoustic scattering from this layer. Up until this point, the majority of research had focused upon fishes and other gas-bearing animals 
(and frequencies < $30 \mathrm{kHz}$ ). Several scientists (Bary (1966) and Barham (1966)) then published results showing poor correlation of acoustic scatter measurements at $12 \mathrm{kHz}$ and zooplankton biomass. It was hypothesized that smaller animals present in the water column, such as crustacean zooplankton, were not detectable at these acoustic frequencies.

It had been found that non-gas-bearing zooplankton could scatter a detectable amount of sound (Smith, 1954). Due to the small size of these zooplankton, higher acoustic frequencies $(>30 \mathrm{kHz})$ were better suited to study these animals. Of the non-gas-bearing biological scatterers, euphausiids were the first scatterers to be identified (Bary and Pieper (1971) and Beamish (1971)), although pteropods were also found to be important scatterers (Hansen and Dunbar, 1971). A few years later, even smaller zooplankton (such as copepods) were suggested as acoustic scatterers that could be detected at even higher frequencies (Castile, 1975).

In a simplified sense, the scattering from objects much smaller than the acoustic wavelength is often negligible. Therefore to see smaller objects (like copepods), higher acoustic frequencies are needed. The drawback to higher frequencies is that they are attenuated in the ocean more rapidly than lower frequencies (Urick, 1983). Much of the early work in echo-detection of biological organisms used lower frequencies in the $10 \mathrm{~s}$ of $\mathrm{kHz}$ (although to most acousticians $10 \mathrm{kHz}$ is a high frequency). Once scientists began to realize that these smaller animals could be detected acoustically, higher and higher frequencies began to be used.

Due to the earlier evidence (and commercial importance) that fish were the dominant scatterers in the ocean, much of the modeling work in acoustic scattering theory focused on bubbles in a fluid. Anderson (1950) found a full modal solution to this problem, and his model is still frequently used today. Scientists realized that one model was not enough to accurately describe the scattering from different types of zooplankton, so several different models have been developed based upon the body type of the zooplankter (fluid-like, elastic-shelled, or gas-bearing).

Over the past two decades, development of zooplankton scattering models has 
become increasingly sophisticated. Driven by a need for more accurate scattering predictions, complex mathematical models of geometric shapes that resemble zooplankton have been created (Stanton et al., 1994a, 1998a; Ye, 1997; Stanton and Chu , 2000b). Scattering predictions from these models have been compared to scattering measurements from actual zooplankton and have shown good agreement (Stanton et al., 1998a). However there are several difficulties in applying these models to field studies of zooplankton scattering. It has been shown that animal orientation relative to the acoustic beam can have a dramatic effect on the scattered energy (Sameoto (1980) and McGehee et al. (1998)). Secondly, few data are available regarding in situ measurements of individual zooplankton target strengths. Laboratory measurements have been made and compared with model predictions, but it is not known how the scattering may differ in the ocean. Currently, many factors (animal behaviors, pressure effects, changes in the physical properties of an animal) affecting zooplankton scattering are not fully understood.

With the increasing sophistication and accuracy of scattering models, combined with significant improvements in acoustic transducer technology, bioacousticians began to focus on a new problem. Since zooplankton scattering spectra are a function of the animal's size and the scattering physics involved, could multiple frequency acoustic measurements be inverted to find the unknown scatterers taxonomic and size distribution? Holliday (1977) provided a mathematical formulation of the problem and this method has been used to predict the size distribution of scatterers (Holliday et al. (1989); Pieper et al. (1990); Napp et al. (1993)). These methods involve the assumption that the zooplankton can be modeled as fluid spheres which limits the accuracy of this method to actual zooplankton (which for the most part are not, sphere-shaped).

Distinguishing between different taxa has not been as thoroughly explored, although some fisheries scientists have used differences in the scattering at two or more frequencies to discriminate certain zooplankton and fish populations (Miyashita and Aoki, 1999; Brierley et al., 1998; David et al., 1999). Additionally, Martin-Traykovski 
(1998) classified three types of zooplankton quite accurately based upon single, broadband insonifications of solitary animals in a laboratory tank. However, before an attempt can be made to apply these inverse methods to field-collected acoustic data; the "forward problem" of estimating the amount of scattering using net tow abundance and scattering model data needs to be better resolved. Without a well-posed model describing the relevant scatterers in the water column, inverse theory will provide erroneous results. Therefore this thesis focuses on improving "forward problem" calculations, with the hope that future work will use these results with inverse theory to provide real-time taxonomic and abundance estimates of zooplankton assemblages.

\subsubsection{Theory}

The use of acoustic surveys to study biological organisms depends upon an understanding of how these animals scatter sound. In essence, a pressure wave is transmitted $\left(P_{\text {trans }}\right)$ into the water column where it strikes an object (the pressure incident upon the target is $\left.P_{m c}\right)$ and the energy is scattered and detected at a receiver $\left(P_{s c a t}\right)$. While the target will radiate energy in all directions, most surveys (and all the experiments in this thesis) measure the amount of sound received at the transmitter, which is called the backscattering case (Figure 1-3).

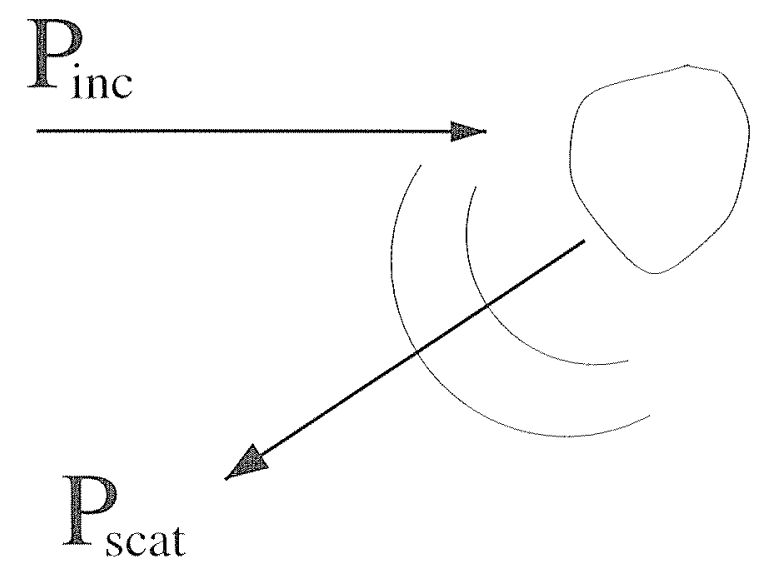

Figure 1-3: Representation of the scattering process from an object. Backscatter occurs when the transmitter and receiver are co-located. 
A mathematical description of the process can be written as

$$
P_{\text {scat }}=P_{i n c} \frac{e^{i k_{1} r}}{r} f
$$

where $k_{1}$ is the acoustic wavenumber in the surrounding medium, $r$ is the distance from the scatterer to the receiver, and $f$ is the scattering function. The scattering function for the backscatter case is $f_{b s}$ and is a function of acoustic wavelength, object size, orientation, shape, and material properties. Therefore efforts to model the scattering from a zooplankton will attempt to define $f$, the efficiency with which the target scatters acoustic energy.

Scattering measurements are usually made in the acoustic far field of both the transmitting transducer and the scattering object. The reason for this is that in the acoustic far field the pressure varies linearly with distance. In the near field of an acoustic radiator, the pressure field can vary widely which makes for a much more complicated analysis. Thus measurements are made in the far field which in practice is generally defined as:

$$
R \gg e \quad \text { and } \quad R \gg \frac{\lambda}{4} \quad \text { and } \quad R \gg \frac{e^{2}}{\lambda}
$$

where $R$ is the distance of the far field from the acoustic radiator, $e$ is the size of the acoustic radiator or target, and $\lambda$ is the acoustic wavelength. The first equation ensures that rays drawn from the object are effectively parallel because the $\frac{1}{R^{2}}$ terms in the pressure equation have a different angular dependence than the $\frac{1}{R}$ terms. The second equation ensures that the pressure wave from an object is very close to a plane wave, and that the phase differences between parts of the wave curvature are smaller than a quarter of a wavelength (thus minimizing destructive interference). The third condition relates the size of the far field to the scatterer size and acoustic wavelength, which ensures that the length of the object (e) is within the first Fresnel zone. In the research presented in this thesis, all the data are collected from the acoustic far field region. A typical zooplankton is roughly $.01 \mathrm{~m}$ in diameter, the acoustic wavelengths 
used range from .002 to $.005 \mathrm{~m}$, and the separation between the scatterer and the receiver is on the order of $.5 \mathrm{~m}$, thus $R \gg e \equiv .5 \gg .01$ and $R \gg \frac{\lambda}{4} \equiv .5 \gg .001$. A more complete discussion on the far field may be found in Junger and Feit (1993).

In addition to the scattering function, more commonly used terms to describe the scattering efficiency of a target are $\sigma_{b s}$, the differential backscattering cross section with units of $\mathrm{m}^{2}$, and $T S$, the target strength measured in $d B$ relative to $1 \mathrm{~m}^{2}$, which are defined as functions of $f$.

$$
T S=10 \log \left|f_{b s}\right|^{2}=10 \log \sigma_{b s}
$$

The above description refers to the scattering from a single object, however if there are multiple scatterers that are insonified then volumetric scattering terms are used such as $S_{v}$, the volume scattering strength, and $s_{v}$, the volume scattering coefficient. For a particular volume, $s_{v}$ is simply the sum of the differential backscattering cross sections of all the scatterers within the volume

$$
s_{v}=\frac{\sum_{i=1}^{n} \sigma_{b s_{i}}}{V}
$$

where $n$ is the number of scatterers in the insonified volume $(V)$ and the units of $s_{v}$ are $\frac{m^{2}}{m^{3}}$. The two volume scattering terms are related by

$$
S_{v}=10 \log \left(s_{v}\right) \quad\left[d B \text { rel } 1 \frac{m^{2}}{m^{3}}\right]
$$

When making zooplankton scattering measurements, the loss of sound energy due to spherical spreading and absorption by the sea water must be determined. These losses are commonly called transmission loss and are often modeled as a logarithmic function of $\mathrm{R}$, the range from the target. In the work in this thesis, a $20 \log (R)$ transmission loss term is used to account for the spherical spreading. Given the shorter ranges used in Chapter Two $(R<1 \mathrm{~m})$, absorption of acoustic energy by sea water is neglected (see Figure 5.5 of Urick (1983)). In the survey data in Chapters 
Three and Four, temperature and salinity measurements are used to calculate $\alpha$, the absorption coefficient and loss of acoustic energy by sea water absorption is calculated. Since the HTI systems used in these chapters output echo integrated volume scattering coefficients for either 0.5 or 1 meter depth bins, transmission and absorption losses are calculated and accounted for in the processing of the echo data for each depth layer.

Finally, multiple scattering effects such as extinction and absorption by the animals are neglected. All zooplankton are weakly scattering objects, thus multiple scattering effects are likely to be small. The complexity added to the scattering analysis by considering multiple scattering theory is beyond the scope of this thesis.

\subsubsection{Scattering Models}

It would be impossible to develop a scattering model for each scatterer in the ocean since there are thousands of species of zooplankton with an enormous variety of shapes and sizes. Therefore bioacousticians categorize zooplankton according to their scattering characteristics. The predominant type of zooplankton are crustaceans and related animals which are fluid-like: meaning that the density and sound speed of the inner parts of the animal are similar to that of the surrounding sea water. Although these animals have a thin shell, it is assumed that this shell does not support a shear wave.

In addition to fluid-like animals, two other acoustically important scattering types are the elastic-shelled and gas-bearing zooplankton. Due to large differences in the density and sound speed of these animals and the sea water (due to the hard aragonite shell of pelagic snails and the carbon monoxide gas inclusion of siphonophores), these animals will scatter a large amount of sound. These three categories of animals are not the only scatterers in the ocean, however they are generally the most acoustically important. Other animals such as non-gas-bearing gelatinous zooplankton are modeled using variations of these three broad categories.

The scattering from a fluid-like animal is described by either simple ray-based 
models (Chapter Two) or by a Distorted Wave Born Approximation numerically calculated by a program written by Dezhang Chu (Chapter Four). The DWBA approach solves for the scattering function, $f$ by evaluating

$$
f=\frac{k_{1}^{2}}{4 \pi} \int_{V}\left(\gamma_{\kappa}-\gamma_{\rho}\right) \exp ^{i 2\left(\vec{k}_{i_{2}}\right) \cdot \vec{r}_{v}} d V
$$

where $V$ is the volume of the entire body described by the position vector $\vec{r}_{v}$, the incident wave number vector is evaluated inside the body $\left(\left(\vec{k}_{i}\right)_{2}\right), \gamma_{\kappa}=\frac{1-g h^{2}}{g h^{2}}, \gamma_{\rho}=$ $\frac{g-1}{g}, h=\frac{c_{2}}{c_{1}}$, and $g=\frac{\rho_{2}}{\rho_{1}}$.

Since most fluid-like zooplankton are cylindrical in shape, various simplifications are made in the implementation of the DWBA approach (see Stanton et al. (1998c)). The fluid-like zooplankton are also grouped for modeling purposes according to body shape, specifically the length-to-width ratio of the animals.

For gas-bearing zooplankton (Chapters Three and Four) there is a complete modal solution to the scattering described by Anderson (1950). This model describes the scattering from the gas inclusion of the animal only, but no other simplifying assumptions are made. Gelatinous zooplankton without gas inclusions, such as medusa and salps, are modeled as weakly scattering fluid-like objects.

The elastic-shelled animals do not have a simple equation that describes their scattering, however like the fluid-like animals, approximations can be made to describe the scattering. In this case, the shell is assumed to be an elastic sphere of the same diameter as the animal. A modal solution is used for low values of $k a$, in the Rayleigh scattering region, and a simple ray solution that describes the specular reflection from the shell is used for large values of $k a$, the geometric scattering region. The geometric solution is adjusted in amplitude so that it equals the modal solution at $k a=1$. In addition to pteropods; eggs and bivalve larvae (if found) are modeled as elastic-shelled objects. The specifics of all of these models are given in Appendix C. 


\subsection{BIOMAPER-II}

With funding from the Office of Naval Research, a new instrument was created by Peter Wiebe and Tim Stanton, assisted by several WHOI engineers and shop personnel. The Blological, Optical, Multi-frequency Acoustical, Physical and Environmental Recorder (BIOMAPER-II) is a unique vehicle that gives us rare insight into the biological and physical structure of the water column. The original BIOMAPER, has been lost and recovered twice on the ocean bottom and is currently stored in the WHOI warehouse. A complete description of BIOMAPER-II and its handling system can be found in Wiebe et al. (submitted).

The instrument consists of three main sensing systems: Acoustics, Video Plankton Recorder (VPR), and Environmental Sensing System (ESS). There is also additional room for other instruments from other scientists. BIOMAPER-II has typically been deployed with several optical and spectral water property sensors used by Heidi Sosik and her group at WHOI.

\section{Physical Description of Instrument}

BIOMAPER-II is $2 \mathrm{~m} \times 0.6 \mathrm{~m} \times 3.8 \mathrm{~m}(\mathrm{H} \times \mathrm{W} \times \mathrm{L})$ and weighs about one ton in air (Figure 1-4). It is composed of a welded aluminum frame designed by Rich Arthur, built by the WHOI shop, and modified by Terry Hammer and Andy Girard. It has mounting brackets inside for numerous instrument pressure housings. The sides are removable plastic panel sections that allow access to the different instruments.

It is tethered to the ship via a $0.68 \mathrm{in}$. armored steel cable which contains three conducting wires for power transmission and three fiber optic lines for data transmission (Figure 1-5). Tom Austin designed the majority of the electronic systems on BIOMAPER-II which allow for communication and control of the instrument.

The control van is a modified ISO shipping container which is placed aboard the ship that is deploying BIOMAPER-II. It contains a power control system, computers for monitoring BIOMAPER-II status, monitors and a VCR to view and record VPR data. In addition, there are computers inside the van for display and recording of 


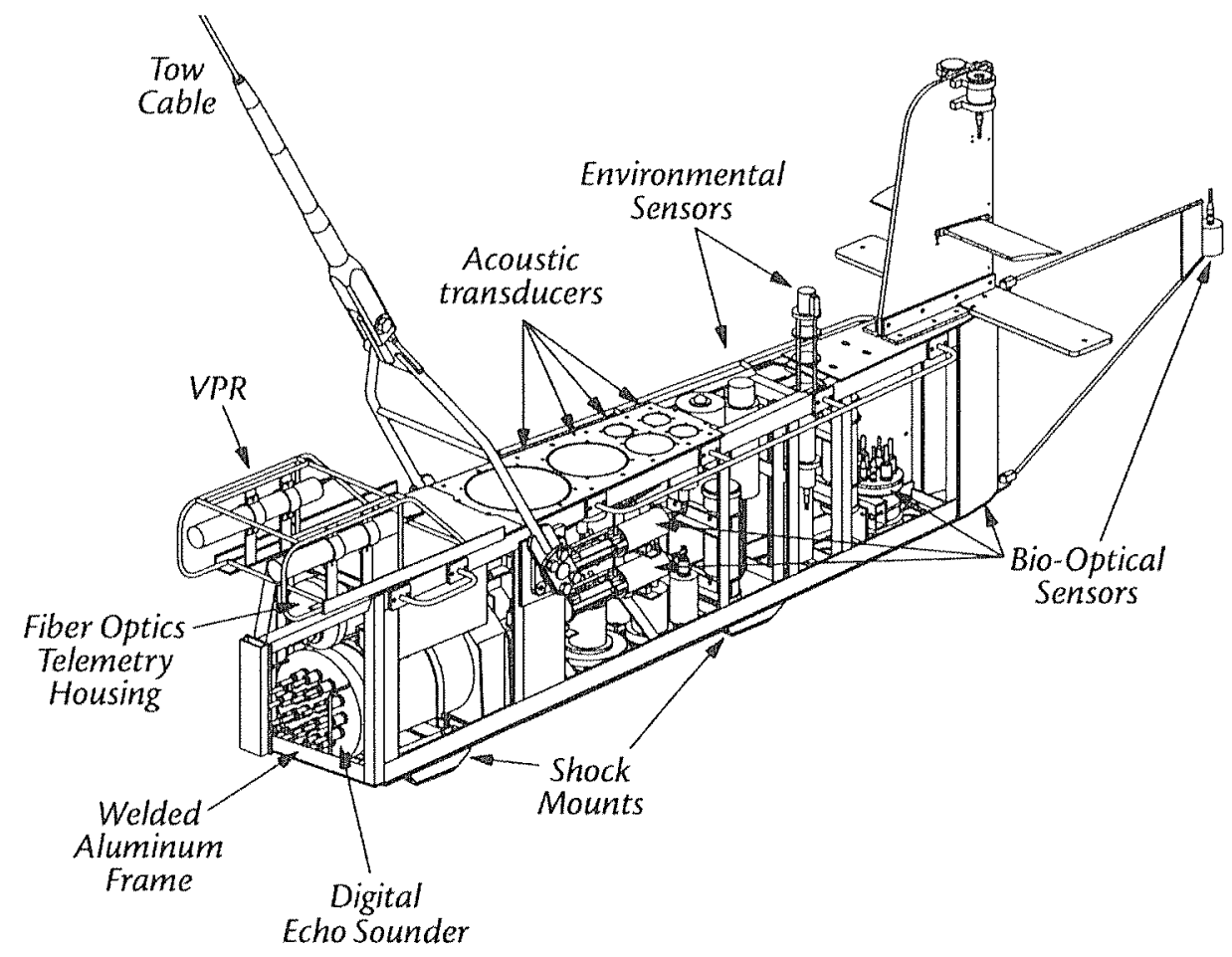

Figure 1-4: A schematic of the BIOMAPER-II instrument showing the different instrumentation systems. (Drawing courtesy of Peter Wiebe)

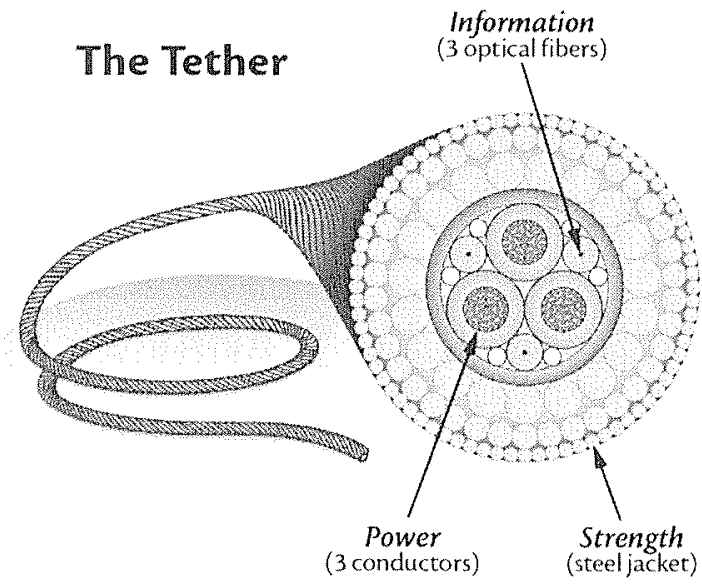

Figure 1-5: A drawing of the BIOMAPER-II 0.68 inch towing cable. Three electrical conductors and three fiber optic cables are surrounded by a steel guard and strength member. (Graphic courtesy of Tim Stanton) 
the acoustic, ESS, VPR, and other sensor systems. There is also room (barely) for scientists and data processing computers.

\section{Multiple Frequency Acoustics}

There are ten acoustic transducers in BIOMAPER-II operating at five different frequencies $(43,120,200,420$, and $1000 \mathrm{kHz})$. Five of the transducers are arranged looking upward, while an identical set of transducers looks downward. This allows BIOMAPER-II to view the entire water column (within the range limits of the transducers) no matter where the instrument is in the water column. The processed acoustic data are recorded on the hard drive of a $\mathrm{PC}$, but the raw acoustic data are recorded on DAT tapes. These tapes can be re-run through the processing system at a later time if further or different processing is warranted.

The acoustic system is manufactured by Hydroacoustic Technology, Inc. (HTI) in Seattle, WA. A unique feature of this instrument is that the multiplexing of the transducers and echo processing occurs in a computer that is mounted in BIOMAPER-II in a titanium pressure housing. This housing contains space for twelve transducer connections of which only ten are currently used; also inside the housing are distributed Digital Signal Processors which perform matched filtering, echo-integration, target detection and tracking. The acoustic system normally collects echo integration data, however since four of the transducer pairs are split-beam (all but the $1 \mathrm{MHz}$ ), they have the ability to do target tracking. This feature allows individual echo target strengths to be found, as well as locating the scatterer three-dimensionally in the water column.

\section{Video Plankton Recorder}

The Video Plankton Recorder (VPR) was conceived and developed by Scott Gallager

and Cabell Davis at WHOI (Davis et al., 1992). This instrument consists of a video camera and a strobe light aimed towards each other (Figure 1-6). The strobe light flashes and illuminates plankton that are in the field of view of the video system. 


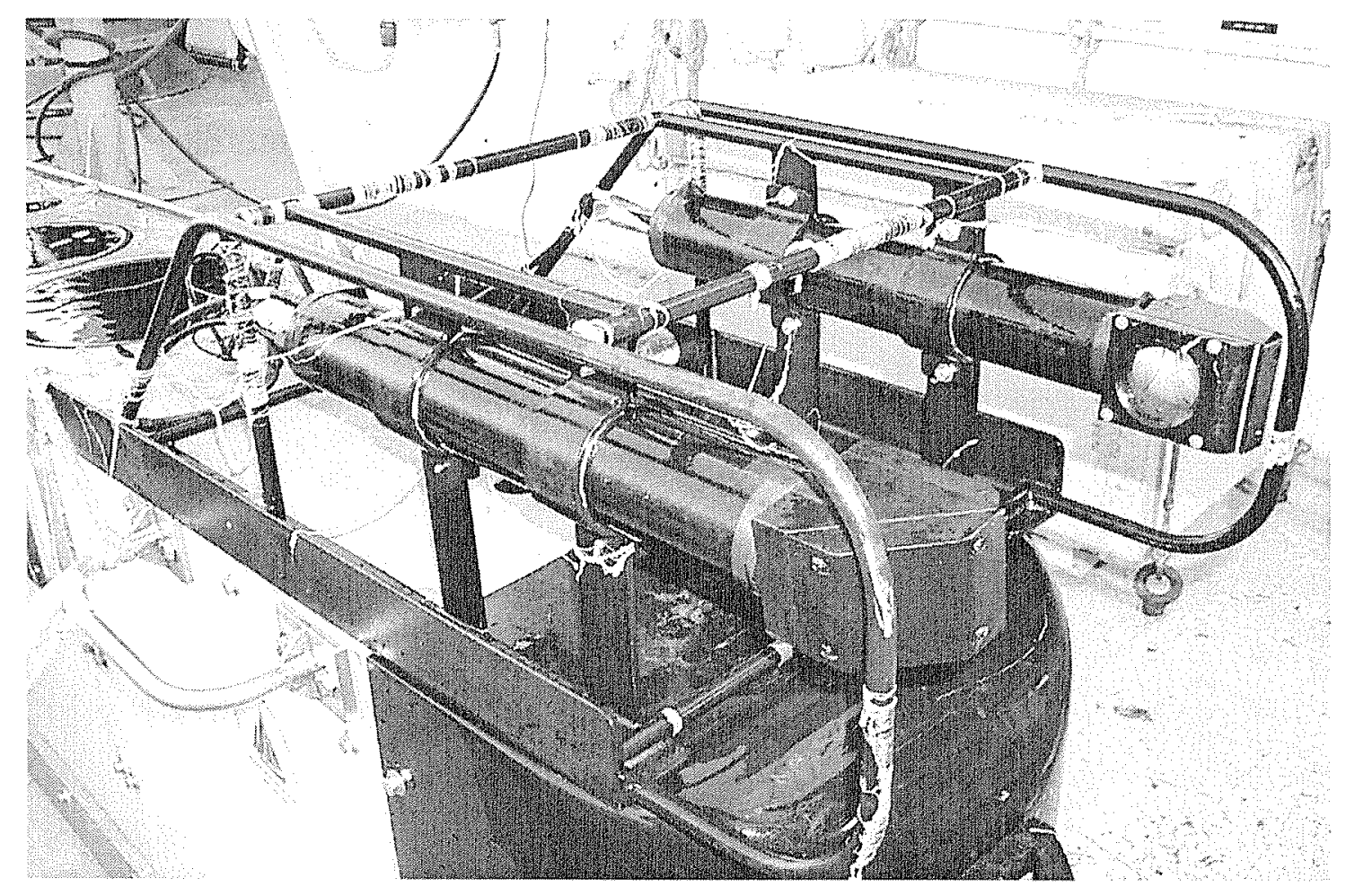

Figure 1-6: The VPR strobe and camera system mounted on the front of BIOMAPER-II. The material on the frame are parts of siphonophores that were caught by the instrument as it was towed through the water. 
The video data are sent up a fiber optic data cable to a VCR and monitors located in the BIOMAPER-II control van. The system is versatile and can be set up to image different volumes of water (Benfield et al., 1996). This instrument is mounted at the front of BIOMAPER-II slightly forward of the main tow body. This allows the instrument to view water that is hopefully undisturbed by the bow wave of the vehicle.

Another component of the VPR system is the Region-Of-Interest (ROI) extraction performed by a hardware and software system run on a Windows NT workstation. This program goes through each frame of the video data that is received and identifies regions of the image that are in focus, mainly by detecting brightness, contrast and edges in the image. The ROI extraction process has a number of variables that determine what the system considers an "in-focus" image. It has been found through experience that to correctly detect different animals with the VPR system that these parameters need to be changed for each animal type. Thus each video tape is run through this process for each type of animal that is of interest to the researcher.

There is currently work being done on an automatic recognition and sorting algorithm that can be used to identify the animal present in each ROI. However, this algorithm is still in the testing stages and therefore the ROIs are currently sorted by hand. This process can be time consuming and tedious, but it does provide zooplankton distribution and composition data for the water column.

The VPR is an ideal complement to the acoustic system. The VPR provides ground truthing by showing exactly what is in the water column, however it only samples a very small fraction of the water column. The acoustic system on the other hand measures acoustic backscattered energy which is related to zooplankton biomass. But the acoustic system covers several thousand cubic meters of water above and below the instrument and thus can investigate a much larger volume of water than the video system. By combining these two systems, there is an ideal data set in which to discover information about zooplankton in the ocean. 


\section{Environmental Sensing System}

The Environmental Sensing System (ESS) is a suite of instruments that have been used previously on MOCNESS net systems (Wiebe et al., 1985) and the Greene Bomber (Wiebe et al., 1996), another acoustic tow body system. It consists of a SeaBird conductivity, temperature and depth sensor; fluorometer; transmissometer; and a down-welling light sensor. These instruments sample at $0.25 \mathrm{~Hz}$ and the data are recorded by a dedicated computer in the BIOMAPER-II control van. They provide information about the physical properties of the water column (as well as information about the phytoplankton via the fluorometer and transmissometer). The ESS data allows insight into the physical structure of the water column. Temperature and salinity microstructure and larger features are evident in these data and may be used to calculate contributions to the acoustic scattering.

\section{Deployment and Use of BIOMAPER-II}

BIOMAPER-II is deployed from the side of a research vessel (Figure 1-7). Tag lines attached to the fore and aft sections of BIOMAPER-II are used in an attempt to control the lateral movement of the vehicle during launch and recovery. Once in the water, the ship's J-frame is fixed in position, and the BIOMAPER-II winch controls cable pay out. A duplicate winch control panel is mounted in the BIOMAPER-II control van, allowing the scientific party to control the ascent and descent of the instrument. The acoustic record provides a very good indicator of how far away the ocean bottom and sea surface are during deployment.

BIOMAPER-II is nomally deployed in a "tow-yo" pattern where the instrument is raised and lowered in the water column while it is being towed. It is typically raised and lowered in the water column at rates of $5-10 \mathrm{~m} / \mathrm{min}$. It can be towed at a ship speed of approximately 6 knots when in tow-yo mode, but while at the surface (approximately $2 \mathrm{~m}$ depth) it can be towed at speeds up to 10 knots. BIOMAPER-II is usually lowered to within $15 \mathrm{~m}$ of the ocean floor and is brought up as near to the surface as possible in order to get a reference reading for the light sensors. A member 


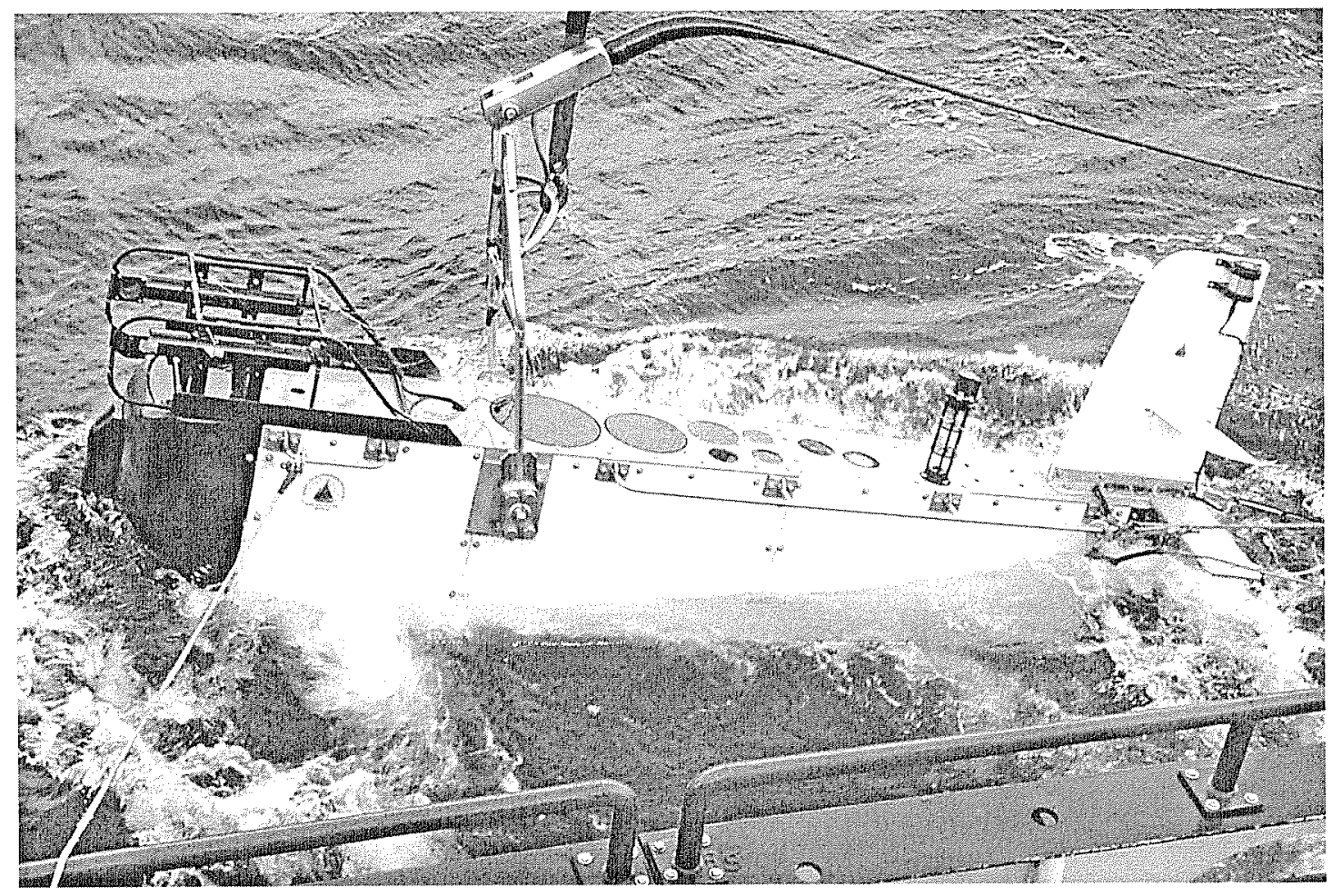

Figure 1-7: BIOMAPER-II being recovered from the deck of the RV Oceanus in December of 1998. The large black circles in the grey steel plate on top of the instrument are the up-looking acoustical transducers. (Photo by Mark Benfield) 
of the scientific party is sent to the deck of the ship to visually verify the position of the vehicle at the top of the tow-yo. In order to prevent damage to the vehicle from surface waves, the vehicle is kept at a $2 \mathrm{~m}$ depth when at the surface.

In 1999, funding was obtained for a motion-compensating handling system for BIOMAPER-II which has made deployment and recovery of the instrument simpler and safer, while at the same time allowing the instrument to be used in sea states that previously would have required recovery of the instrument (Figure 1-8). The handling

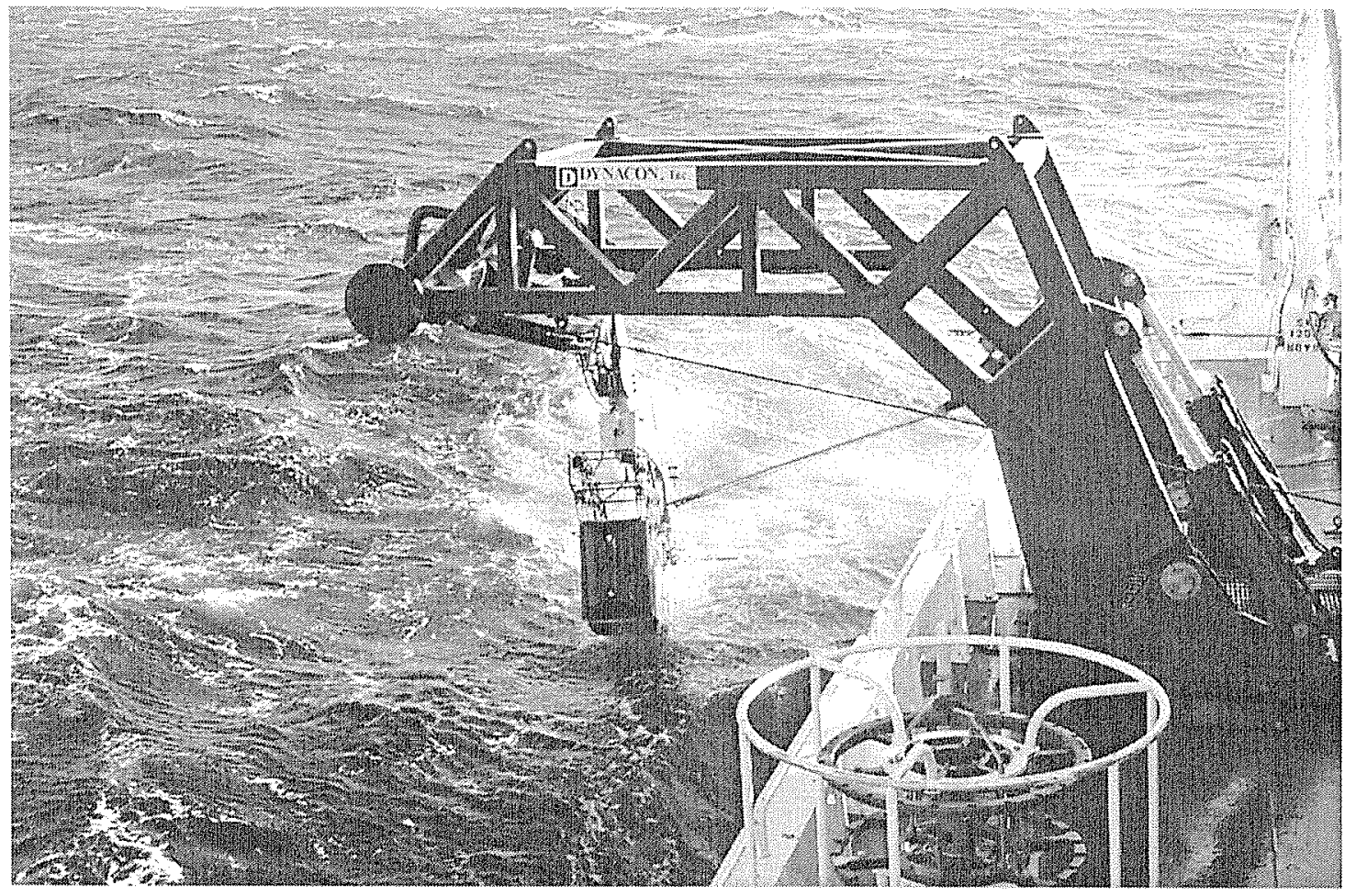

Figure 1-8: The handling system manufactured by Dynacon, Inc. recovering BIOMAPER-II during the October 1999 cruise on the RV Endeavor. In sea states like this, BIOMAPER-II could not have been deployed or recovered with the old handling system. (Photo by Peter Wiebe)

system is mounted to the deck of the ship (once the deck has been reinforced!) and can be controlled either on deck or by a remote control unit located inside the van. The key to this handling system, built by Dynacon, Inc., is the slack-tensioner which consists of seven sheaves (four sheaves are mounted at the bottom, three are mounted to a vertically moving assembly driven by hydraulic cylinders) which either take up 
or let out cable to eliminate large spikes in the tension of the towing cable. Up to 7 $m$ of cable can be taken up or let out by the slack-tensioner system, compensating for motion of the ship relative to the sea surface.

Recovery of the instrument involves slowing the ship to roughly one knot. Tag lines connected to air tuggers on the deck of the ship are hooked into the guard rails on the side of BIOMAPER-II. These lines are kept taut, while the instrument is brought above the side of the ship. The handling system then moves the vehicle inboard and lowers it onto the deck of the ship. The instrument is then secured to the deck of the ship using web straps.

\section{Data Integration}

One of the difficulties in processing the wealth of data provided by BIOMAPER-II is that it is outputed in three different formats. There is the acoustic record which contains a time stamp, latitude, longitude, and echo integration data for various depth bins. The ESS record contains time, latitude, longitude, pressure, temperature, salinity, and numerous other sensor output. And finally the VPR data which consists of numerous images with a time stamp. The combination of these different data sets is normally based upon the time stamp. Therefore it is important that the clocks of these three systems be synchronized. The ESS and acoustics computers are synchronized automatically, and the VPR system is regularly checked to ensure that it is in agreement with the other systems. The use of GPS (Global Positioning Satellite) time ensures that there is minimal deviation among the systems.

Another difficulty in using these data is that BIOMAPER-II currently produces approximately a gigabyte of data every hour. Once processed, this amount is brought down by a factor of ten, however this is still a great amount of data to manipulate and archive. Even though computer technology has advanced at quite a remarkable rate, creating plots of twelve hours of acoustic data still requires minutes of computer time rather than seconds. Because of this, careful selection of regions of interest to explore is very important. While analysis can be performed on a basin-scale, it is difficult to 
view this much data with any sort of quantitative measure. The qualitative images of the different basins are necessary though in illuminating the many differences in the acoustic structure (and the zooplankton) of each of the basins.

Additionally, direct sampling of the zooplankton is done with a MOCNESS (Multiple Open and Closing Net and Environmental Sensing System) (Wiebe et al., 1985). These samples combined with the previously described instruments provide three complementary types of data: acoustic, video and net. While each of these methods has its drawbacks, the combination of these data sets offer the best possible opportunity for sampling of the Gulf of Maine ecosystem. 


\title{
Chapter 2
}

\section{Effect of Animal Orientation on Acoustic Estimates of Zooplankton Properties}

Art is delayed echo.

- George Santayana

\begin{abstract}
It is well known that the behavior of zooplankton and, in particular, their orientation dramatically affect the level of backscattered acoustic energy. In order to quantify these effects, laboratory data from two different types of animals were collected. The data involved broadband $(350-650 \mathrm{kHz})$ acoustic signals insonifying animals whose orientation was varied over the range $0-360^{\circ}$ in $1^{\circ}$ increments. The animals were from two major anatomical groups: fluid-like (decapod shrimp; Palaemonetes vulgaris) and elastic-shelled (periwinkles; Littorina littorea). The data were analyzed both in the time domain (with pulse compression processing) and the frequency domain. The analysis gives estimates of the changes in average target strength for different ranges of animal orientation that can be expected in the natural environment. A
\end{abstract}


$3 \mathrm{~dB}$ difference was found in target strength measurements of the shrimp averaged over distributions centered around broadside and end-on incidence. In addition, size estimates from pulse compression processing of the broadband echoes were made for both fluid-like and elastic-shelled animals. These results show the necessity of animal orientation information for the proper interpretation of acoustic backscattered energy.

\subsection{Introduction}

Measurements of acoustic scattering in the ocean are used by biologists to determine the abundance of zooplankton in the water column (Holliday et al., 1989; Wiebe et al., 1996). However, there is not a simple relationship between the amount of sound scattered and the number and type of animals. Originally, the fluid sphere scattering model by Anderson (1950) was used to model zooplankton. This spherical model enabled scientists to estimate animal biomass in the ocean and also to obtain information about the size distribution of the animals (Holliday et al., 1989; Greenlaw, 1979; Holliday and Pieper, 1995). Research has shown that the simple model of a sphere is inadequate for modeling some types of zooplankton and that the orientation of the animal can have a profound effect on the scattering (Greenlaw, 1977; McGehee et al., 1998). In an attempt to solve this problem, scientists have developed more realistic models of the scattering physics for several types of zooplankton (see reviews in Holliday and Pieper (1995), Foote and Stanton (2000)).

Although significant progress has been made on the development of scattering models, much still needs to be done. For example, a crucial element in understanding the scattering is its dependence upon the angle of orientation of the animal. The average level of scattering for some orientations can be predicted using available theory (Stanton et al., 2000a). For other orientations, the models must be advanced in order to make reliable predictions. Given the fact that the models may be valid only for a certain range of orientations, a scattering analysis may involve supplementary laboratory data for animal orientations which the models do not accurately describe.

This paper describes a study where laboratory data involving two types of zoo- 
plankton were collected at high angular resolution and over an octave bandwidth. The data are processed in order to predict the effects of animal orientation on the scattering levels and on acoustic estimates of animal size.

\subsection{Theory}

Acoustic backscatter from a zooplankter is determined by the differences in density and sound speed between the animal and the surrounding fluid, the animal's shape and the animal's size relative to the acoustic wavelength. By using simple geometric shapes to represent the animal, mathematical scattering models for several different types of plankton have been developed (Stanton et al., 1998b). This paper discusses two types of zooplankton that have very different physical attributes and are thus modeled separately: those with fluid-like bodies and elastic-shelled animals.

Both of these animals have been successfully modeled with ray-based methods previously (Stanton et al., 1998b, 2000a). Ray-based methods are an approximation that is valid only in the geometrical scattering region $(k a \geq 1)$, where $k$ is the acoustic wavenumber and $a$ is a characteristic size of the animal, generally its radius. In this study ka ranges from $3-8$. Acoustic rays are used to represent scattering from specific features or mechanisms of the scatterer. The advantage of this approach is that accurate representations of the scattering from an animal can be made by using the contributions from the most dominant mechanisms, while the disadvantage is that these results are only applicable in the geometric scattering region.

Common to both types of animals is the fact that an echo due to a single insonification of an individual animal will have multiple returns that can overlap in the time domain. The different returns are due to the various features of each animal that scatter sound, or multiple returns from a single feature. The overlap is due to the small size of the animals and the relatively long pulse length of the acoustic wave that strikes the animal. With sufficiently high bandwidth in the insonifying signal and matched filter signal processing, the individual echoes can be resolved. Without analyzing each of the echoes that scatter from a single animal, one would be 
measuring the constructive or destructive interference pattern from the overlapping echoes.

\subsubsection{Fluid-like Animals}

Zooplankton that are fluid-like in their body composition occur throughout the oceans. Common types of fluid-like animals include krill, amphipods, and decapod shrimp. These animals all have a morphology roughly similar to the species (Palaemonetes vulgaris) studied herein.

Fluid-like animals generally have a very thin outer shell or exoskeleton (which is assumed not to support a detectable shear wave) enclosing the body of the animal which has physical characteristics that are similar, but not necessarily the same, as those of the surrounding medium. There is strong evidence that the scattering from the above-mentioned animals occurs primarily from reflections from the outer boundary of the animal. For example, previous studies involving krill and shrimp (Chu and Stanton (1998), Stanton et al. (1998a)) have found that near broadside incidence there are generally two main echoes from the animal: one at the front interface of the body, and a second echo from a wave that propagates through the animal's body, reflects off the interface at the far side of the animal, propagates back through the animal's body and finally is detected by the receiver (Fig. 2-1a).

For the simple case of broadside incidence, the time delay between the arrivals of the two echoes provides useful information. The time delay will be directly proportional to the distance the wave travels inside the animal. Thus the animal's diameter can be estimated if the speed of sound in the interior of the animal is assumed to be constant. The ratio of sound speed of a fluid-like animal relative to that of the surrounding medium $\left(h=\frac{c_{\text {animal }}}{c_{\text {seawater }}}=1.0279\right)$ found by Foote $(1990)$ for a euphausiid is used. The animal's orientation relative to the acoustic wave will also affect the time delay. If the animal is at broadside incidence to the acoustic wave then the time delay can be converted directly to an estimate of the diameter of the animal. At oblique angles, the conversion of time delay information to diameter estimates re- 

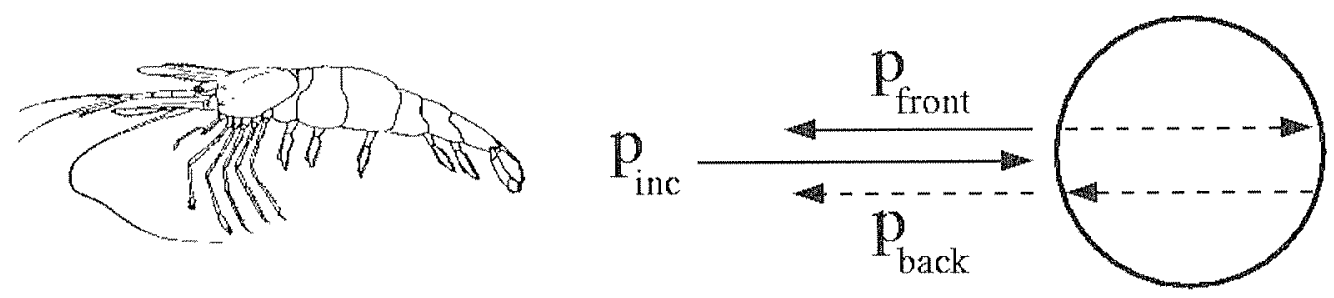

(a)
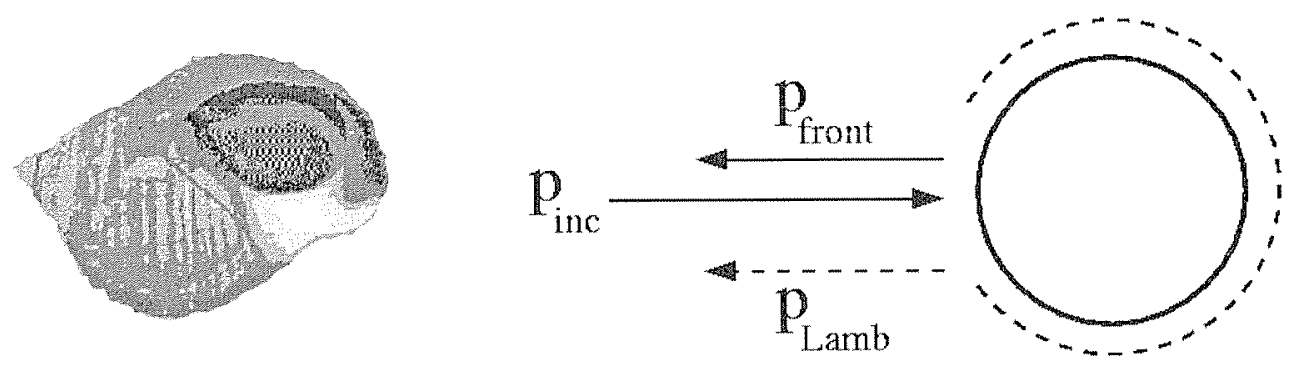

(b)

Figure 2-1: Schematic drawing of certain scattering mechanisms for the (a) shrimp and (b) snail. $\mathrm{P}_{\text {front }}$, PLamb and Pback are the pressure fields from the front interface reflection, the Lamb wave and reflection off the back interface of the animal, respectively. 
quires knowledge of the orientation and the use of simple trigonometry. This method is not applicable at all angles due to limitations of the scattering model near end-on incidence and is further complicated by the presence sometimes of more than two echoes (Stanton et al., 1998b). These echoes are likely to occur from reflections from parts of the animal other than the front and back interface (such as the rostrum or telson.

\subsubsection{Elastic-shelled Animals}

Periwinkles (Littorina littorea) are bottom dwelling snails that are commonly found in inter-tidal zones. They are similar in morphology to certain planktonic pteropods (e.g. Limacina retroversa) which can be important acoustic scatterers in the water column (Stanton et al., 1994a; Benfield et al., 1996; Wiebe et al., 1997). The pteropods are very difficult to study individually in the laboratory due to their small size (diameters are generally less than $1 \mathrm{~mm}$ ). Since there is strong evidence that periwinkles and planktonic snails have similar scattering characteristics (Stanton et al., 2000a), periwinkles were used in this study.

Scattering from elastic-shelled animals is characterized by a very strong echo specularly reflected by their hard shell. A previous study (Stanton et al., 2000a) has found that strong secondary echoes are also present. The secondary echoes have been determined to be from two different mechanisms: 1) a Lamb wave that travels along the animal's shell, partially circumnavigating the animal, and then returning in the backscatter direction (Fig. 2-1b), and 2) echoes from within the opercular opening. When the opening faces the transducer, the acoustic wave can travel inside the opening, scatter off the back wall and return to the transducer. Scattering from the animal itself is very weak relative to that from the shell since the animal has a density and, presumably, a sound speed close to that of sea water. Therefore, the effect of the animal will be ignored and only scattering from the shell will be considered.

The scattered energy from the periwinkle has been shown to vary greatly with the orientation of the shell (Stanton et al., 2000a), due to the complexity of the shape of 
the animal's shell. Although somewhat cone-shaped, the shell is actually a tube that is coiled upon itself. Therefore the surface of the shell has both varying thickness and surface roughness. In fact, depending on the orientation of the animal, the scattering characteristics can even change the number of echoes that are reflected. When the back of the opercular opening of the animal is facing the acoustic wave, there is no Lamb wave detected. This may be the result of energy not being able to couple to the Lamb wave due to the solid angle of the opercular opening being larger the coupling angle required to excite the Lamb wave (Kargl and Marston, 1989).

It is possible to gather information from the arrival times of the different waves that scatter from elastic-shelled animals. If the secondary arrival is from a Lamb wave, then the time delay between the two echoes corresponds to the circumference of the animal (and thus its diameter). It should be noted that the second arriving ray in our two ray model is referred to as the Lamb wave ray. This is somewhat misleading since a Lamb wave can not propagate in the water column, only along an elastic surface. Thus the second arriving ray travels through the water column at a velocity $c$, strikes the elastic shell and the energy is coupled into a Lamb wave which propagates at $c_{\text {Lamb. }}$. The Lamb wave after circumnavigating the shell, then couples, or launches, back to the water column where the wave is then detected by the receiver.

However there are complicating factors to this simple approach: the speed of the Lamb wave, the distance the Lamb wave travels along the shell, and the arrival of other Lamb waves that have circumnavigated the shell more than once. Lamb wave velocities for these frequencies and shell dimensions ( $k a \sim 4-8)$ are generally subsonic, however there have not been any direct measurements of their speed in calcium carbonate shells. Previous work (Stanton et al., 2000a) has estimated their average velocity as approximately one third of the speed of sound in sea water. While there is a dispersive relationship in Lamb wave velocities (Zhang et al., 1992), this average value has been used previously with good results and will be used in this analysis.

These Lamb waves do not travel a complete circumnavigation around the shell. 
There is a specific angle $\left(\theta_{\text {Lamb }}\right)$ relative to the axis of the shell where the wave starts or "lands" on the shell, as well as leaves or "launches" from the shell and returns to the water column. Therefore the arc traveled by the Lamb wave on a spherical shell is $2 \cdot\left(180^{\circ}-\theta_{\text {Lamb }}\right)$. For sub-sonic waves, $\theta_{\text {Lamb }}=90^{\circ}$ (Kargl and Marston, 1989). Lamb waves that have circumnavigated the shell multiple times will be delayed in time by approximately $\frac{\pi r}{c_{\text {Lamb }}}$ where $\mathrm{r}$ is the radius of the shell $(.003 \mathrm{~m})$ and $c_{\text {Lamb }}=500 \frac{\mathrm{m}}{\mathrm{s}}$. Therefore the expected time delay between multiple-circumnavigating Lamb waves is $\sim 19 \mu$ s, which is less than our pulse length of $200 \mu \mathrm{s}$. Returns from multiple circumnavigations of the Lamb wave should be detected, however our analysis will only use the first two waves that are detected (the specular reflection, and the first Lamb wave if it is excited).

\subsubsection{Pulse Compression Processing}

Pulse compression (PC) processing is a signal processing technique that has recently been used to analyze backscattered echoes from biological targets (Chu and Stanton (1998), Stanton et al. (1998a)). It is identical to a matched filter which has been used in radar and sonar analysis for quite some time (Turin, 1960). This method can improve the signal-to-noise ratio and involves the cross-correlation of a received signal with a filter which is typically a replica of the transmitted signal. For our application, the filter is the received signal of the calibration of the system.

The pulse compression processing is described fully in Chu and Stanton (1998), however the basic approach is as follows. A transmitted signal, $s_{0}(t)$, is scattered by some object and is detected by a receiver. The received signal, $r(t)$, is a time-delayed, amplitude-modulated version of the transmitted signal convolved with the scattering function of the object, plus noise

$$
r(t)=\frac{1}{r^{2}} f_{b s}(t) * s_{0}\left(t-t_{0}\right)+n(t)
$$

where $r$ is the distance from receiver to the target (assuming that the transmitter and receiver are co-located), $f_{b s}$ is the scattering impulse response of the target, $t_{0}$ is 
the time delay, and $n(t)$ is a noise function.

The convolution process is defined by the convolution integral where

$$
y(t)=x(t) * h(t)=\int_{-\infty}^{\infty} x(\tau) h(t-\tau) d \tau
$$

In the case of a matched filter: $x(t)$ is the transmitted signal, $h(t)$ is the system response of the scatterer, and $y(t)$ is the matched filter. The method used in this analysis differs because the system response of the scatterer is not known, so instead the received calibration signal, which is the transmitted signal convolved with electronic and transducer effects, is used as the matched filter.

The properties of the transmitted signal have an important effect on the matched filter output. Normally, if a constant frequency signal (such as a pure tone) is transmitted then the range resolution of the target is $\frac{c}{2 T_{0}}$, where $T_{0}$ is the duration of the transmitted signal. However, if matched filtering processing and broadband signals are used, then the received signals are compressed in time and the range resolution is $\frac{c}{2 B W}$, where $B W$ is the bandwidth of the transmitted signal and is equal to $\mu T_{0}$ where $\mu$ is the chirp rate of a linear frequency modulated signal. With higher signal bandwidth, the peak of the impulse response of the matched filter becomes narrower, and thus closely spaced returns are able to be better resolved in time. Unfortunately power and transducer limitations generally prevent very high bandwidth signals from being transmitted. However, a "chirp" (or linearly frequency modulated) signal can provide a larger bandwidths while keeping signal duration times relatively low (low signal duration times are often the result of power limitations). A chirp signal has a frequency that increases linearly with time (a human whistle that rises in pitch is a type of "chirp" signal) and these signals are often used by echo-locating animals such as dolphins or bats because of the advantages mentioned previously.

The frequencies used in this study are higher than those typically used in acoustic field surveys of zooplankton. These frequencies do not travel as far through the water column (due to absorption of energy by sea water), however they are in the geometric scattering range for most zooplankton $(k a>1)$. Differences in scattering 
spectra in the geometric scattering regime can be used to distinguish between different

types of zooplankton (Martin-Traykovski, 1998) and it is for this reason that the high frequencies were used. Some field surveys (such as those discussed in Chapter Four) have begun to use these higher frequencies as a means to distinguish between different types of scatterers and therefore it is important to be able to model accurately the scattering from zooplankton at these higher frequencies.

\subsection{Experimental Methods}

The experimental procedures used in this study have been described previously (Stanton et al. (1998a), Stanton et al. (2000a), Stanton (1990), Chu et al. (1992)); so only a brief summary will be presented. An array of sixteen pairs of transducers configured in a bistatic arrangement and aimed in the horizontal direction was placed in a large fiberglass tank filled with sea water. For the data presented herein, a "chirp" signal, centered at $500 \mathrm{kHz}$, was generated with a bandwidth of approximately $300 \mathrm{kHz}$. The pulse length was $200 \mu$ sec. After the acoustic wave scattered off the planktor, the echo in the backscatter direction was detected by a receiving transducer, identical and adjacent to the transmitting one. After amplification, the received signal was digitized and displayed on a digital oscilloscope, and then transferred to a computer for analysis. For each experiment, an individual animal was suspended with a thin monofilament line (59 $\mu \mathrm{m}$ diameter). A single line was used for the periwinkle shell, while a two-line configuration was used with the live shrimp. The two-line "harness" restricted the shrimp's movement and permitted better control of its orientation. The top of each tether was attached to the axis of a stepper motor which was used to rotate the animal.

The decapod shrimp was tied such that the animal was within $20^{\circ}$ of lying flat on its side in the horizontal plane. The shrimp rotated in this plane so that scattering information in the dorsal/ventral aspect was obtained (Fig. 2-2). The periwinkle was tied such that it could be rotated for apex/opercular opening scattering. The planes of rotation were selected as to provide animal orientations that may be found 
in the field. The decapod shrimp remained alive during the experiment, and the periwinkle experiment involved scattering from the shell only (the animal was removed beforehand). Great care was taken to prevent accumulation of bubbles onto the tether and bodies of the animals.

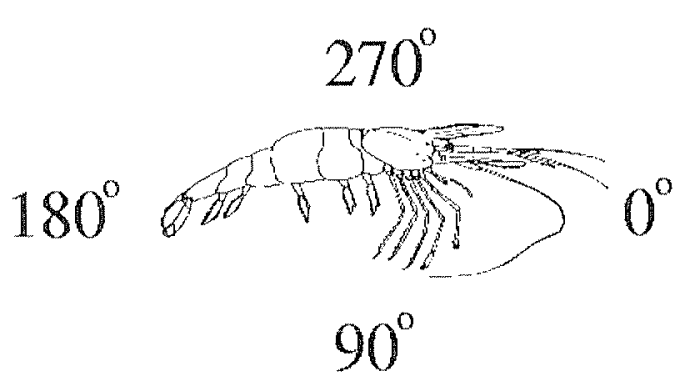

(a)

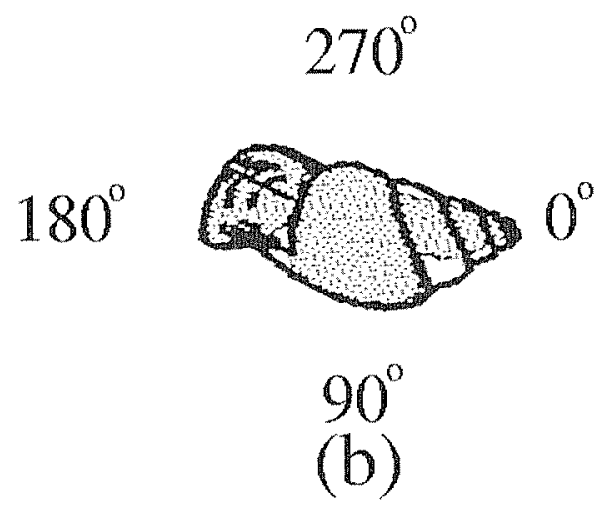

Figure 2-2: Orientation of incident acoustic wave relative to the (a) shrimp and (b) snail. $0^{\circ}$ incidence is approximately "head-on" and "apex-on" incidence for the two animals respectively.

Post-processing of the data involved the use of a digital bandpass filter (to remove noise) and then PC processing. The compressed echoes were then processed by an automatic peak detection algorithm to determine the location and magnitude of the reflections from the different facets of the animal. The largest and generally earliest occurring echo is referred to as the primary peak, and the remaining peaks are ordered by their arrival time (secondary, tertiary).

\subsection{Results}

\subsubsection{Fluid-like Animal}

A total of ten decapod shrimp were used in the experiment with echoes collected at $1^{\circ}$ increments over two complete rotations $\left(720^{\circ}\right)$ of the shrimp's body. Results were similar for all animals, so only results from one animal $(\# 6)$ are presented herein (Table 2.1). The raw echo is difficult to interpret since it is composed of overlapping echoes from at least two reflections from the animal (Fig. 2-3a), however the PC 
output clearly distinguishes several distinct echo arrivals from the animal (Fig. 23b). The magnitude of the primary and secondary arrivals (as well as the raw echo level) vary regularly with angle of orientation (Fig. 2-4). The peaks of the echo levels correspond to when the shrimp is at broadside incidence $\left(\theta=90^{\circ}, 270^{\circ}, 450^{\circ}, 630^{\circ}\right)$ to the insonifying wave.
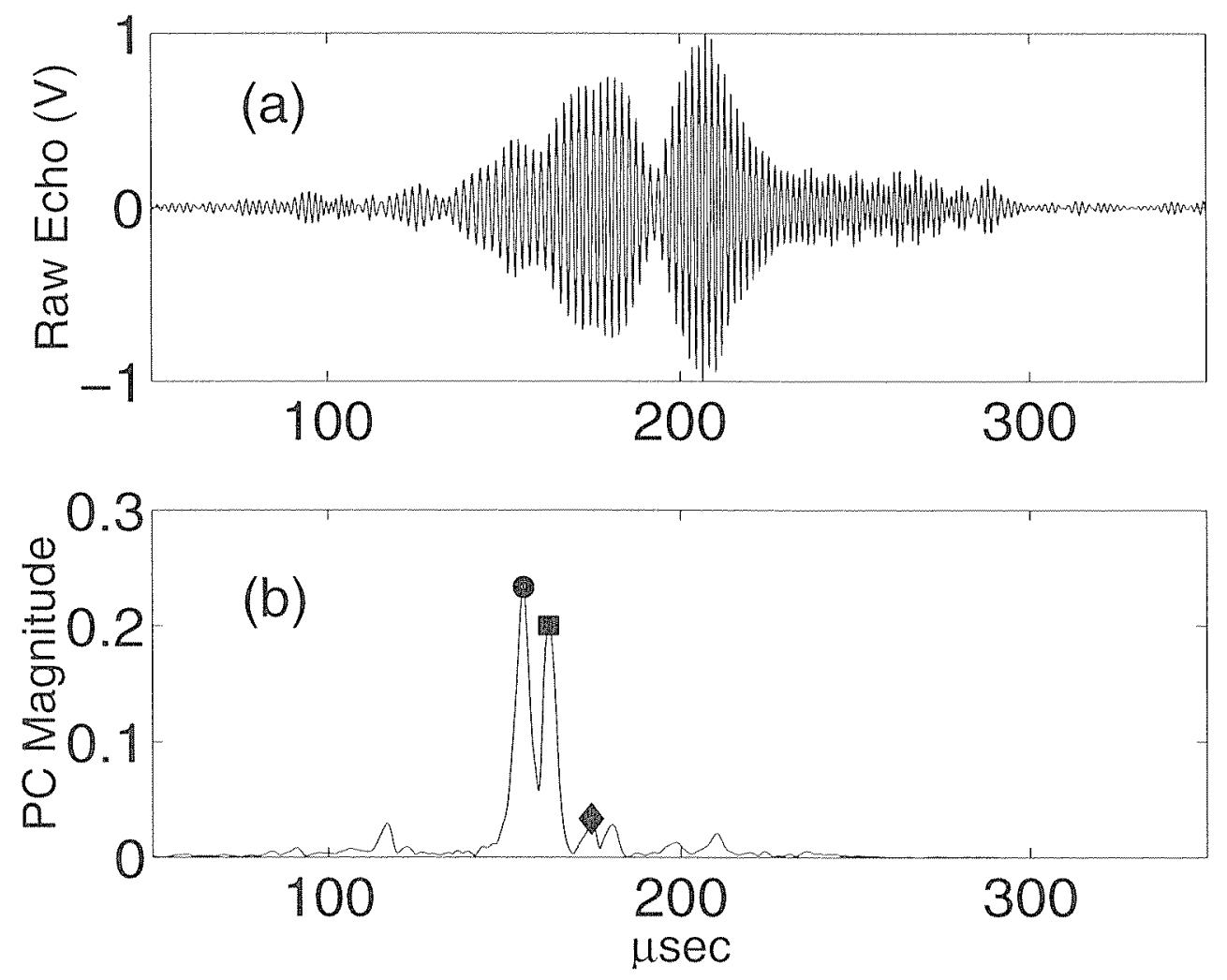

Figure 2-3: Example echo from decapod shrimp: (a) raw echo voltage (b) envelope of pulse compression output showing the primary $(O)$, secondary $(\square)$ and tertiary $(\diamond)$ returns.

Information about the animal can be extracted from the timing of the PC echo arrivals. Estimates of the animal's dimension were made from the time delay between the primary and secondary echo arrivals for all angles of orientation (Figure 2-5). These estimates assume a uniform sound speed for the inside of the animal's body. The mode of this distribution is $4.2 \mathrm{~mm}$ which agrees very well with the measured animal diameter of $4.15 \mathrm{~mm}$ at the widest section. However there are estimates of 


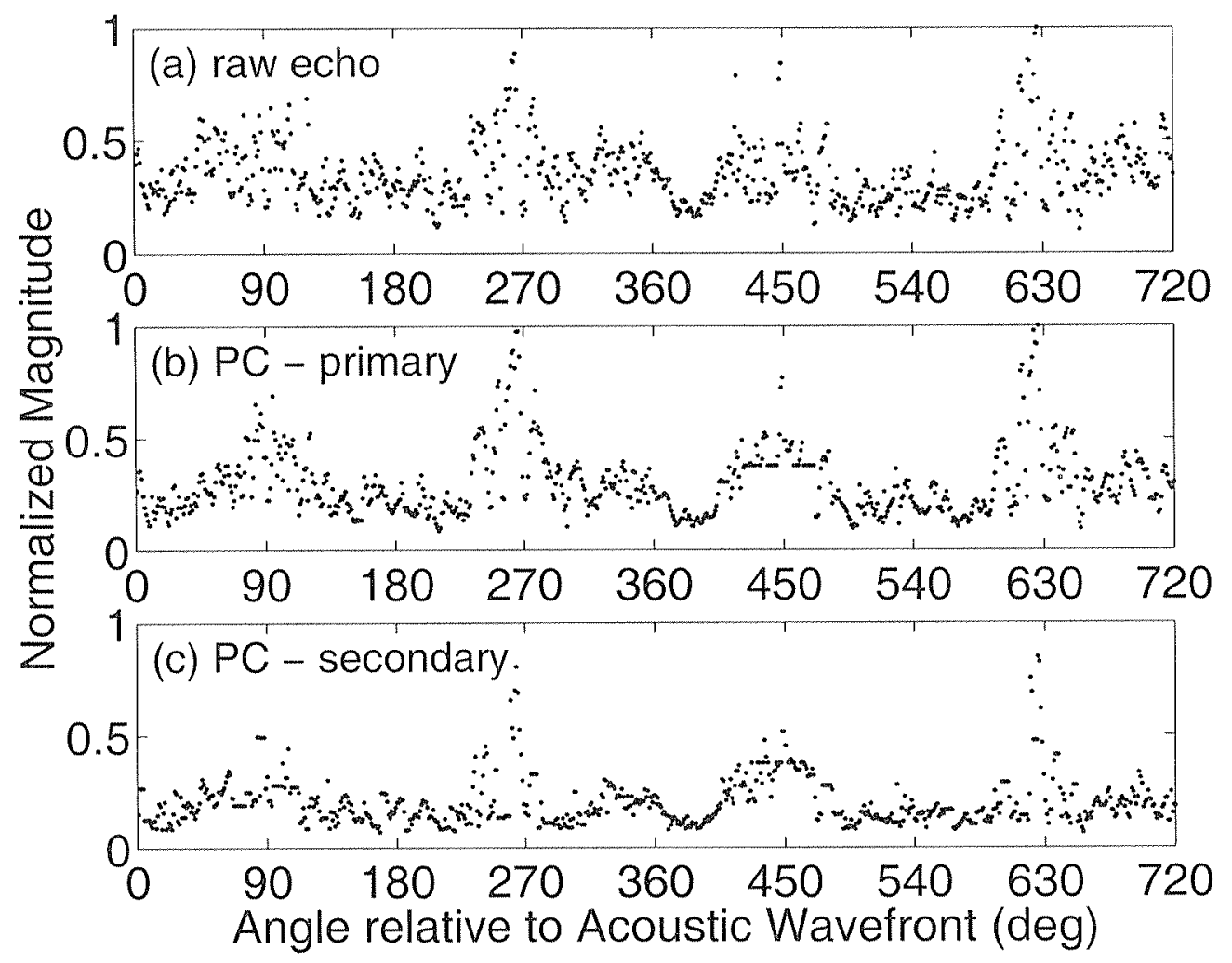

Figure 2-4: Normalized magnitude of (a) raw echo voltage, (b) primary and (c) secondary peaks of PC output for shrimp \#6. Odd multiples of $90^{\circ}$ correspond to broadside incidence, while even multiples of $90^{\circ}$ correspond to end-on incidence. Magnitudes of the secondary peaks are normalized to the largest overall value of the primary return. 
Table 2.1: Dimensions of the animals used in the experiment. Length of the shrimp measured between anterior of the eye and end of the telson. Dianeter of the shrimp is the average of the measurement for each of the first two thoracic segments. Length of the periwinkle is the maximum tip-to-tip distance. Diameter of the periwinkle was measured between the plane containing the face of the opercular opening and the outer point of the shell on the opposite side of the shell.

\begin{tabular}{|l|l|l|l|}
\hline Common Name & Species & Length & Diameter \\
\hline Grass Shrimp (\#06) & Palaemonetes vulgaris & $19.37 \mathrm{~mm}$ & $4.15 \mathrm{~mm}$ \\
\hline Periwinkle (\#01) & Littorina littorea & $6.2 \mathrm{~mm}$ & $3.9 \mathrm{~mm}$ \\
\hline
\end{tabular}

the animal's size that are smaller than the animal's dimensions. It is possible that they are associated with reflections from the narrower or tapered part of the animal's cephalothorax and telson. There are several outlying estimates of the animal's dimension that are larger than the physical length of the animal $(19.37 \mathrm{~mm})$. These may be reflections from other parts of the animal such as the legs, rostrum, or telson. However, the main "body" of the histogram ends at approximately $15 \mathrm{~mm}$ which is a reasonable estimate of the length of the animal if the telson (which is quite thin) is excluded. These results suggest that a single animal insonified by multiple pings can be sized accurately using information from multiple features of the compressed echo from the animal.

In order to study the effects of different orientations, theoretical data sets were created. An "orientation distribution" was created by selecting a range of angles and then calculating what the echo statistics would be from a group of animals with a uniform distribution of angles within the specified range. Since it is very difficult to determine the orientation for every single animal within a zooplankton swarm, scattering data from a single animal (of known size) but with differing orientations is used to simulate what a echo-sounder would detect from scattering from a group of zooplankton in the field. The majority of sonars used in zooplankton surveys are downward looking.

The echoes from these sonars would insonify shrimp from the dorsal/ventral plane rather than the left/right side plane that a side-looking sonar would encounter. Unfortunately, there is no definitive work on the orientation of decapod shrimp or euphausiids in the field. However, Sameoto (1980) studied the orientation of euphausiids 


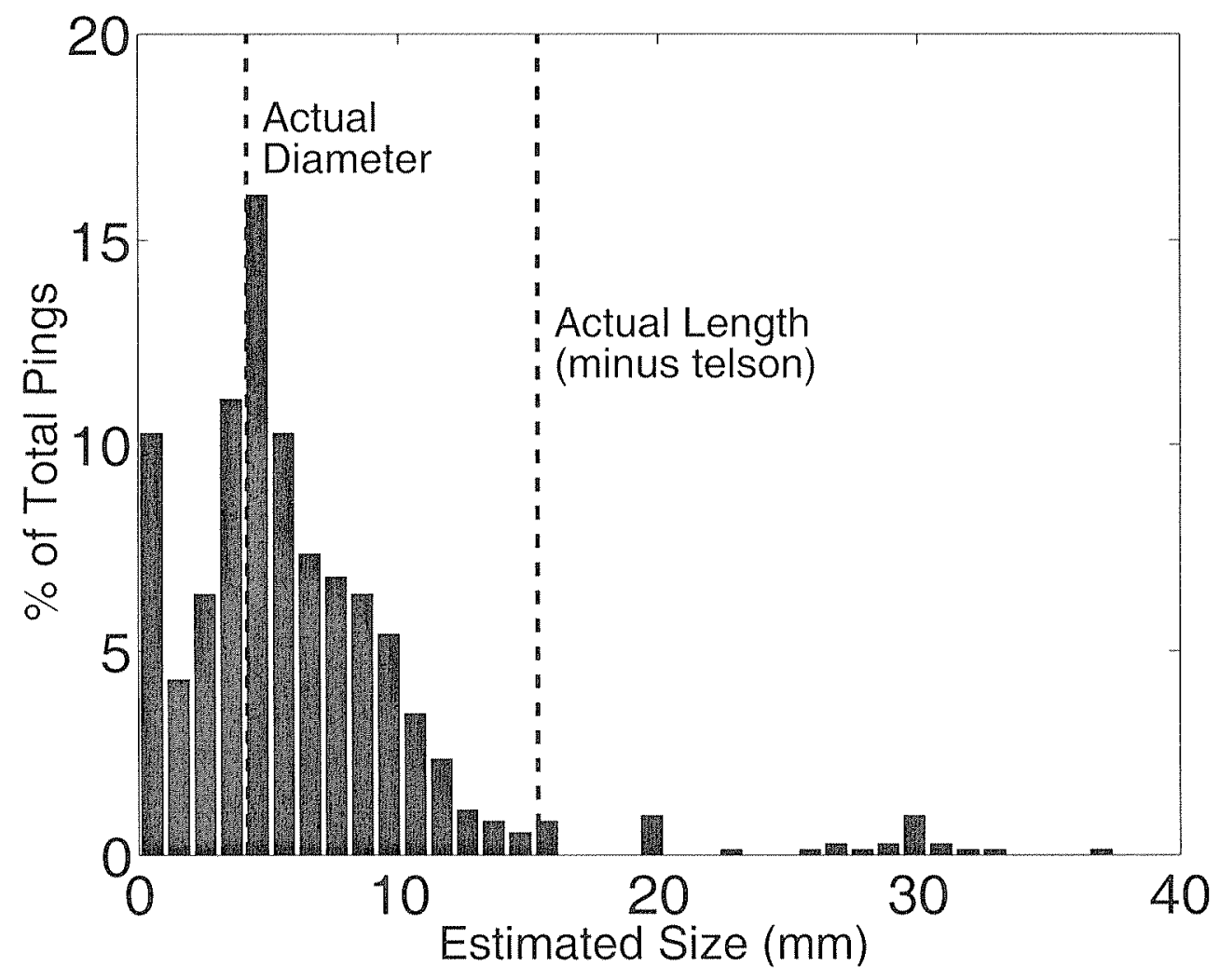

Figure 2-5: Histogram of uncorrected estimated diameter of shrimp \#6 for pings from all angles of orientation $\left(0-720^{\circ}\right)$. Mode of distribution is $4.2 \mathrm{~mm}$. Measured diameter was $4.15 \mathrm{~mm}$. 
in the water column from photographs taken from a net system. There was generally a broad range of orientations for the animals, however at certain times (1500h and $2400 \mathrm{~h}$ ), his results show a preferred orientation of the animal being slightly head-up in the water column. Also, several other studies (Kils, 1981; Miyashita et al., 1996) show that krill exhibit this head-up position for certain behaviors. We use this orientation distribution as a basis for our modeling of what a downward looking sonar would detect. Since it is likely that animal orientation distributions change throughout the day depending upon the behavior of the animal (vertical migration and feeding for example), other ranges of orientations are studied. Multiple scattering effects are ignored in this case since zooplankton are weak scatterers. Size estimates (Table 2.2) and target strength values (Table 2.3) were calculated for the various theoretical data sets.

The data for different orientation distributions show that there are changes in the mean value and variance of the target strength of the animal (Table 2.3). Although the variances in the Target Strength are substantial (due to the wide frequency range used and the frequency dependence of the scattering), there are changes in the mean Target Strength between the various orientation distributions. The differences are largest $(\sim 3 \mathrm{~dB})$ between the distributions centered around end-on and broadside incidence. While these differences are smaller than those observed at lower frequencies (McGehee et al., 1998), they are large enough to cause substantial changes in biomass estimates (the goal of most zooplankton surveys). These data indicate the need for more information on in situ animal orientation.

Additionally, the theoretical data sets were used to determine how animal behavior may change estimates of the animal's size from time-delay measurements from the PC processing (Table 2.2). An "uncorrected" diameter estimate $(d$ ') was found by converting the time delay between the primary and secondary peaks in the pulse compressed echo $(\tau)$ to a length without using any orientation information

$$
d^{\prime}=\frac{\tau \cdot c_{\text {animal }}}{2}
$$


Table 2.2: Acoustic estimates of animal diameter for different theoretical animal orientation distributions. All sizes are in millimeters. The mean and the mode for each orientation distribution is given. Uncorrected diameters are calculated from time delays between primary and secondary arrivals. For the shrimp, a corrected diameter was determined by taking into account the known orientation in the estimation of the diameter. Measured dimensions of the animals are given in Table 2.1. There is no diameter estimate for the periwinkle in the back-of-shell case due to the absence of a detectable Lamb wave. There is no corrected diameter for the shrimp at end-on incidence since the trigonometric model is not applicable (NA) at those angles.

\begin{tabular}{|c|c|c|c|c|c|c|}
\hline Animal & $\begin{array}{l}\text { Orientation Distribution } \\
\theta\end{array}$ & $\begin{array}{l}\text { Estim } \\
\text { (no co } \\
\text { Mean }\end{array}$ & $\begin{array}{l}\text { d Diam. } \\
\text { Motion) } \\
\text { Mode }\end{array}$ & $\begin{array}{l}\text { Estim } \\
\text { (with } \\
\text { Mean }\end{array}$ & $\begin{array}{l}\text { d Diam. } \\
\text { rection) } \\
\text { Mode }\end{array}$ & $\begin{array}{l}\text { Meas. } \\
\text { Diam. }\end{array}$ \\
\hline Shrimp & $\begin{array}{l}\text { Down-Looking Sonar } \\
290^{\circ} \pm 20^{\circ}\end{array}$ & 4.9 & 3.7 & 4.3 & 3.0 & 4.15 \\
\hline Shrimp & $\begin{array}{l}\text { Uniform } \\
1-360^{\circ}\end{array}$ & 6.0 & 4.2 & 4.2 & 3.0 & 4.15 \\
\hline Shrimp & $\begin{array}{l}\text { Broadside Incidence } \\
90^{\circ} \pm 20^{\circ}, 270^{\circ} \pm 20^{\circ}\end{array}$ & 4.2 & 4.3 & 4.1 & 4.3 & 4.15 \\
\hline Shrimp & $\begin{array}{l}\text { End-On Incidence } \\
0^{\circ} \pm 20^{\circ}, 180^{\circ} \pm 20^{\circ}\end{array}$ & 6.2 & 5.1 & NA & NA & 4.15 \\
\hline Periwinkle & $\begin{array}{l}\text { Down-Looking Sonar } \\
250^{\circ} \pm 40^{\circ}\end{array}$ & 6.3 & 7.3 & $\mathrm{NA}$ & $\mathrm{NA}$ & 6.2 \\
\hline Periwinkle & $\begin{array}{l}\text { Uniform } \\
1-360^{\circ}\end{array}$ & 6.8 & 5.0 & NA & $\mathrm{NA}$ & 6.2 \\
\hline Periwinkle & $\begin{array}{l}\text { Apex Incident } \\
20^{\circ} \pm 10^{\circ}\end{array}$ & 5.6 & 5.3 & $\mathrm{NA}$ & NA & 6.2 \\
\hline Periwinkle & $\begin{array}{l}\text { Opercular Incident } \\
270^{\circ} \pm 10^{\circ}\end{array}$ & 6.1 & 7.1 & NA & NA & 6.2 \\
\hline Periwinkle & $\begin{array}{l}\text { Back of Shell Inc. } \\
180^{\circ} \pm 10^{\circ}\end{array}$ & NA & NA & $\mathrm{NA}$ & $\mathrm{NA}$ & 6.2 \\
\hline
\end{tabular}

A "corrected" animal diameter $(d)$ was calculated taking into account orientation using a simple trigonometric relationship

$$
d=d^{\prime} \cdot \sin (\theta)
$$

where $\theta$ is the angle of the animal relative to the acoustic wavefront as shown in Fig. 2-2. The mean and mode of the corrected and uncorrected diameter estimates for the various orientations were found. For the uncorrected estimates of diameter, the mode of the distribution had a better agreement with the measured diameter of the shrimp, however when geometric information was included to correct the estimate, the mean 
Table 2.3: Target Strength statistics for different orientation data sets of shrimp and periwinkle scattering

\begin{tabular}{|l|l|l|}
\hline Animal & Orientation Distribution $(\theta)$ & $\begin{array}{l}\text { TS } \pm \sigma_{T S}^{2} \\
350-650 \mathrm{kHz}\end{array}$ \\
\hline Shrimp & Down-Looking $\left(290^{\circ} \pm 20^{\circ}\right)$ & $-91.1 \pm 2.8$ \\
Shrimp & Uniform $\left(1-360^{\circ}\right)$ & $-90.5 \pm 6.4$ \\
Shrimp & Broadside $\left(90^{\circ} \pm 20^{\circ}, 270^{\circ} \pm 20^{\circ}\right)$ & $-88.5 \pm 9.8$ \\
Shrimp & End-On $\left(0^{\circ} \pm 20^{\circ}, 180^{\circ} \pm 20^{\circ}\right)$ & $-91.7 \pm 4.7$ \\
\hline Periwinkle & Down-Looking $\left(250^{\circ} \pm 40^{\circ}\right)$ & $-57.8 \pm 1.3$ \\
Periwinkle & Uniform $\left(1-360^{\circ}\right)$ & $-57.7 \pm 8.6$ \\
Periwinkle & Apex $\left(20^{\circ} \pm 10^{\circ}\right)$ & $-63.8 \pm 2.6$ \\
Periwinkle & Opercular $\left(270^{\circ} \pm 10^{\circ}\right)$ & $-57.0 \pm 0.8$ \\
Periwinkle & Back of Shell $\left(180^{\circ} \pm 10^{\circ}\right)$ & $-53.8 \pm 4.3$ \\
\hline
\end{tabular}

value was closer to the measured diameter. In the case of broadside incidence the acoustic estimates of diameter and the measured value agreed almost exactly, for the other animal behaviors the acoustic estimates tended to over-estimate the measured value by approximately 5\%. There was no calculation of estimated diameter for the end-on incidence case since the theoretical model used is not applicable at those angles.

To examine the accuracy of the theoretical two-ray model and the orientation corrections to the diameter estimates; a comparison of uncorrected diameters, corrected diameters and theoretically predicted uncorrected diameters was done (Fig. 2-6). For a range of angles from head-on $\left(0^{\circ}\right)$ to end-on $\left(180^{\circ}\right)$ incidence with $1^{\circ}$ increments, mean time delays were calculated and then converted to uncorrected diameter estimates. These values were then corrected using the orientation of the animal and Equation 2.4. Using the measured value of the animal's diameter, a theoretical prediction for the uncorrected diameter is made with the formula

$$
d_{\text {theoretical }}^{\prime}=\frac{d_{\text {measured }}}{\sin \theta}
$$

Obviously this equation is not valid for orientations near end or head-on incidence since the diameter prediction goes to infinity. A comparison of these two estimates and prediction shows that the valid range of orientations for the uncorrected and 
corrected estimates are approximately $\pm 50^{\circ}$ and $\pm 35^{\circ}$, respectively, from broadside incidence. The uncorrected estimates follow the theoretical trend for uncorrected data within about $\pm 60^{\circ}$ from broadside incidence.

\subsubsection{Elastic-shelled Animal}

Six periwinkle shells (Littorina littorea) were used in the experiment. Results were similar for all cases and the data shown are from animal \#1 (Table 2.1). Similar to the decapod shrimp analysis, individual scatterings are difficult to identify in the raw echo, but are clearly shown in the PC processed echo (Fig. 2-7). The interference between the reflection from the front interface and the other echoes can change the overall amplitude of the reflected signal. The major structure in the polar plots of echo versus orientation for the raw echo voltage and the primary peak magnitude are broadly similar (Fig. 2-8). There are differences in the smaller scale structure between the two plots which indicate the interference effect of the secondary echo. These secondary echoes are readily apparent in the PC processed signal and can provide information about the size of the animal.

Stanton et al. (2000a) stated that for certain orientations, the time delay between the primary and secondary peak corresponds to a circumnavigating Lamb wave that travels subsonically around the shell and then couples, or launches, from the shell into the water. At these values of ka, the Lamb wave speed that gave the best fit to their data was $c_{\text {Lamb }}=500 \frac{\mathrm{m}}{\mathrm{s}}$. Lamb wave speeds are frequency dependent, however this average value can provide useful information. The term "Lamb wave speed" refers only to the velocity that the Lamb wave propagates along the shell, when the "Lamb wave"-ray is in the water column it travels at the velocity $c$. Using this speed, the dimension of the periwinkle studied can be extracted from the acoustic data. The diameter $(d)$ of the shell can be found from the formula

$$
d=\frac{2 \cdot \tau \cdot c_{\operatorname{Lamb}}}{\pi}
$$

where $\tau=$ time delay between primary and secondary pulse compressed echo 


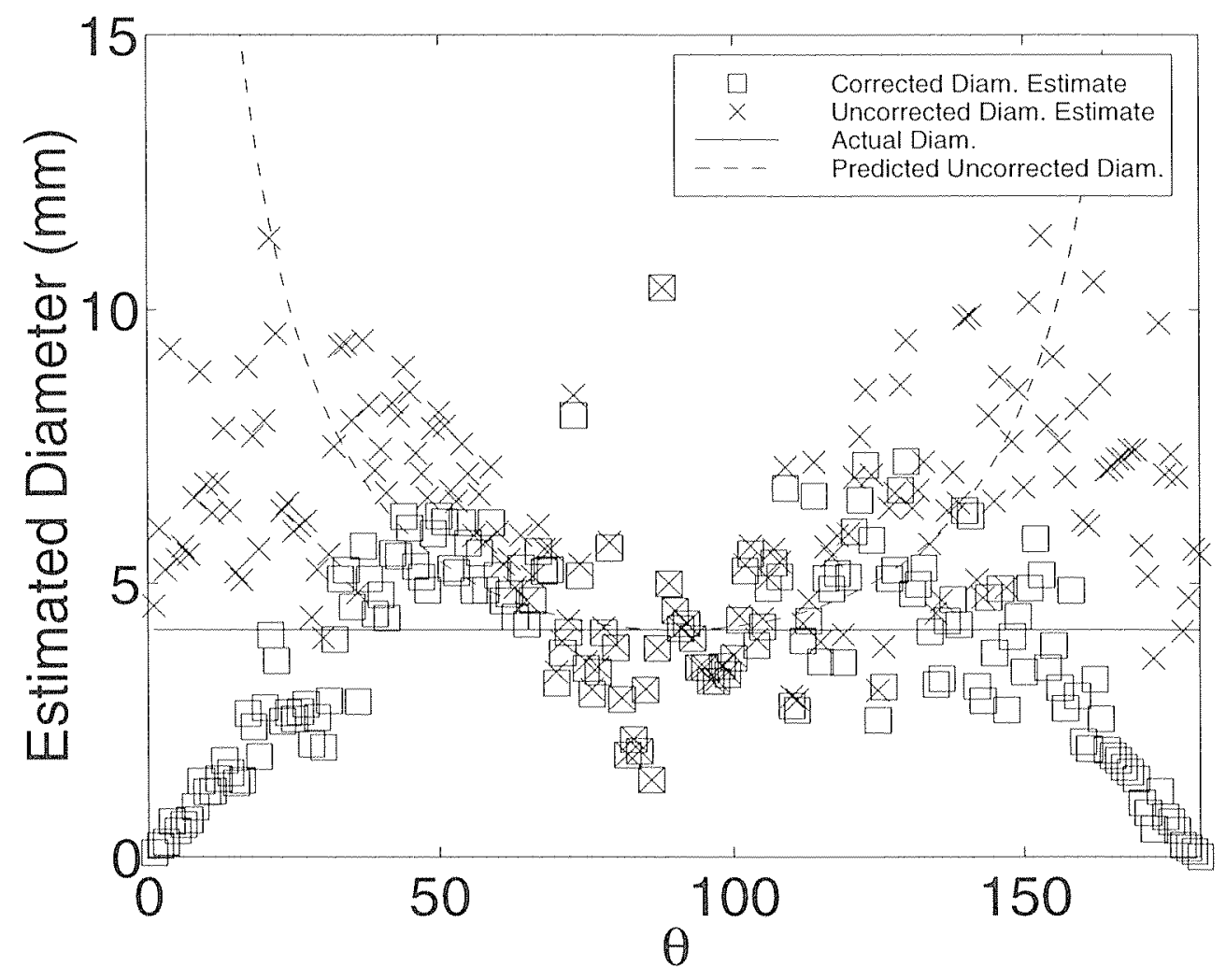

Figure 2-6: Uncorrected $(x)$ and corrected $(\square)$ diameter estimates of the shrimp from the time delay between primary and secondary $\mathrm{PC}$ peaks. For each $1^{\circ}$ increment, four data points from two complete revolutions of the animal $\left(0-720^{\circ}\right)$ were averaged to provide a data set covering $180^{\circ}$ from head-on to end-on incidence. For example, the data point at $30^{\circ}$ is the mean of the values collected at $30^{\circ}, 330^{\circ}, 390^{\circ}$, and $690^{\circ}$. Dashed line is a theoretical prediction of the uncorrected diameter estimate using the measured animal diameter of $4.15 \mathrm{~mm}$. The corrected and uncorrected scattering model appear to produce reasonable estimates within the range of $\pm 50^{\circ}$ and $\pm 35^{\circ}$, respectively, from broadside incidence. 

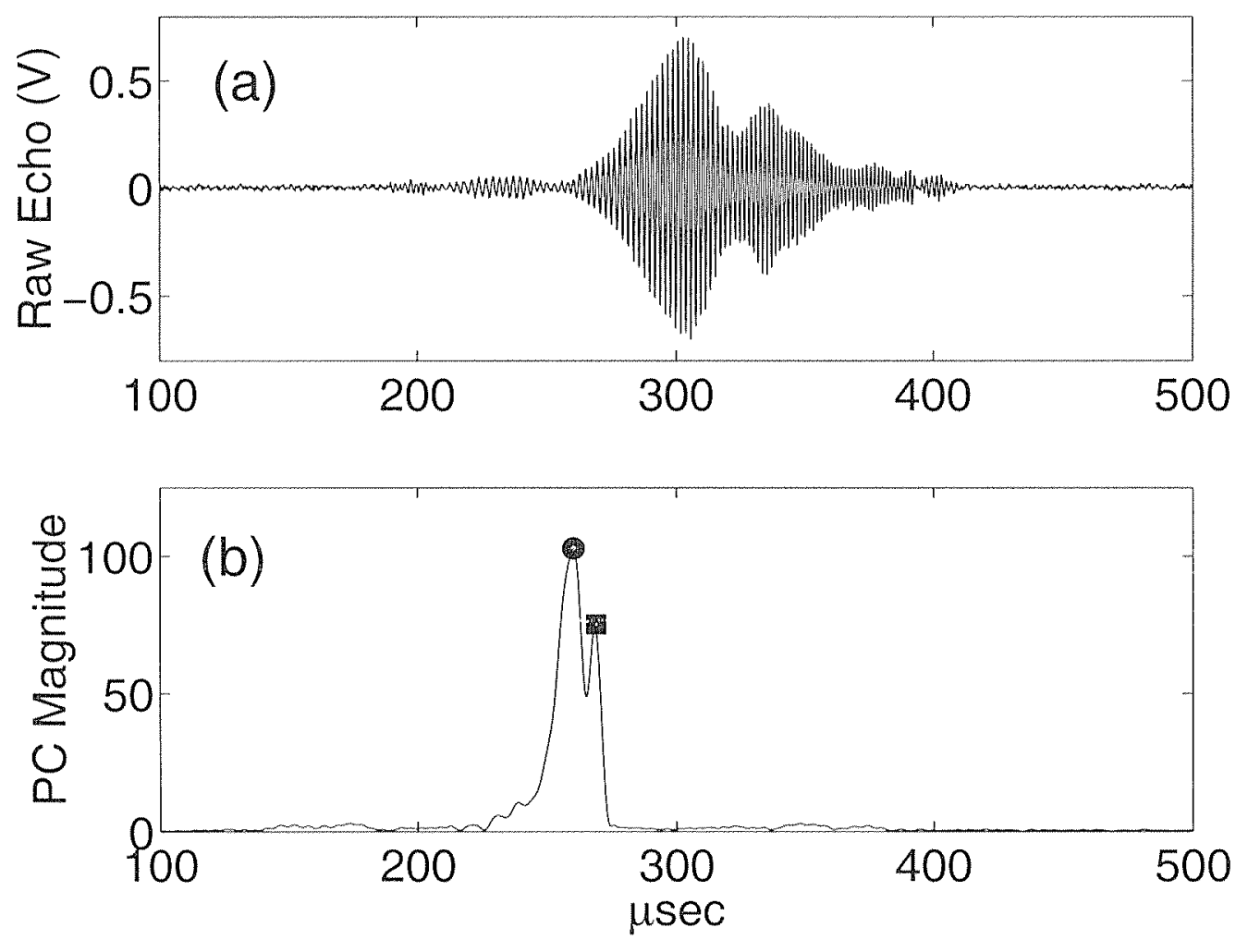

Figure 2-7: Example echo from periwinkle: (a) raw echo voltage and (b) pulse compression output showing the primary $(\bigcirc)$ and secondary $(\square)$ returns. 


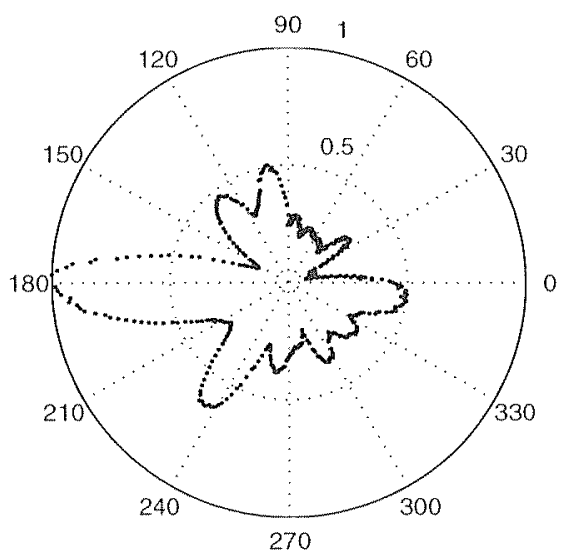

(a) Raw Voltage

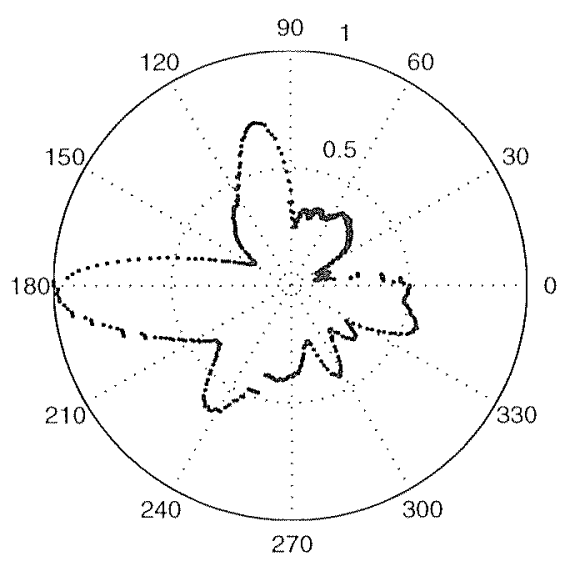

(b) PC - primary peak

Figure 2-8: Polar plot of scattering by a periwinkle versus angle of orientation: (a) the normalized raw echo amplitude and (b) the normalized primary PC peak are shown. 
arrivals, and the distance traveled by the Lamb wave is halfway around the shell (Kargl and Marston, 1989).

There is a fair amount of variation in the time delay between the primary and secondary peaks from the periwinkle (Fig. 2-9), which could be explained by Lamb wave speeds varying due to changes in shell thickness (Stanton et al., 2000a). However, there is a sharp peak in the distribution at $18.5 \mu \mathrm{sec}$. Using Equation 2.6, the estimated diameter of the shell is found to be $5.9 \mathrm{~mm}$ (compared to the measured diameter of $6.2 \mathrm{~mm}$ ). Thus the dimension of the animal can be estimated using a two ray model for the scattering and $\mathrm{PC}$ processing to resolve the multiple returns.

As was done for the shrimp, theoretical data sets were created to model different sonar viewing angles. Planktonic pteropods have been found to have a preferred orientation in the water column. For both feeding and swimming, these animals position themselves with their opercular opening pointing mostly vertically upward (Morton, 1954; Gilmer and Harbison, 1986). Therefore, with a downward looking sonar, the opercular opening would be generally aimed towards the transducer and the apex would be aimed downward and to the side. In addition to the down-looking sonar data set; theoretical data sets were created for a uniform distribution, apex-upward, opercular opening-upward and back of shell-upward distributions. These different data sets show how the resulting size estimates (Table 2.2) and target strengths (Table 2.3) can change with various animal orientations.

It is apparent given the range in target strength values $(\sim 10 \mathrm{~dB})$ that large errors in estimates of animal populations can occur if the wrong orientation distribution were used in modeling these animals. However all of the various distributions provided a similar range of estimates for the size of the animal. This indicates that the Lamb wave speed and path are relatively stable across the range of orientations, at least when averaged over the distance traveled around the shell.

Given that the scattering mechanism for the elastic-shelled animal is affected by the complex shape of the animal, a simple model that does not take into account the actual geometry of these shells will not provide a realistic representation of the scattering 


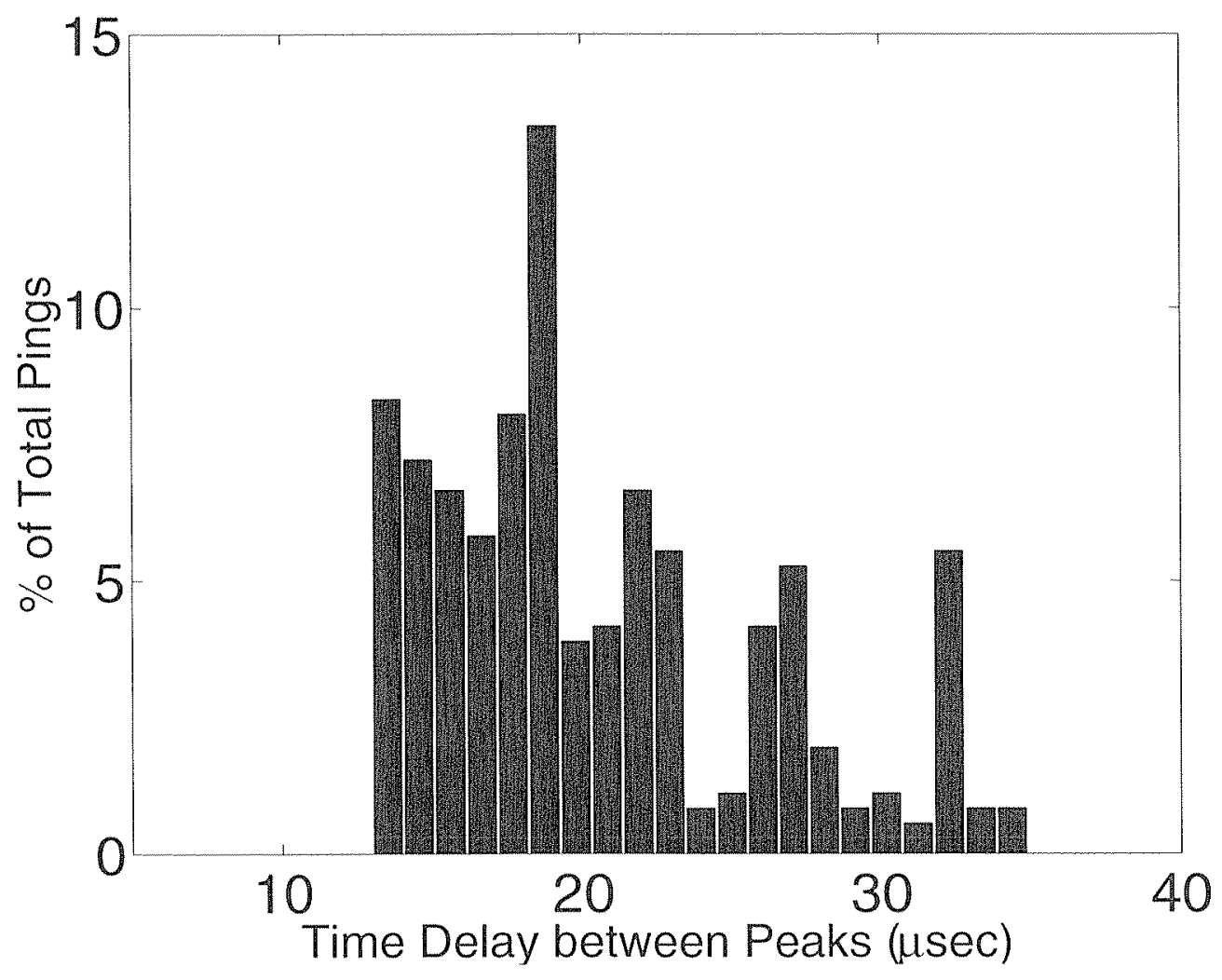

Figure 2-9: Histogram of time delay between primary and secondary PC peaks of the periwinkle. Mode of distribution is $18.5 \mu \mathrm{sec}$, which corresponds to an estimated diameter of $5.9 \mathrm{~mm}$. Measured animal diameter is $6.2 \mathrm{~mm}$. There is a threshold of $13 \mu \mathrm{sec}$ in the peak detection algorithm. 
from these animals. However, a simple ray model may offer a good approximation for describing the broad trends in how animal orientation can affect the backscattered energy.

\subsection{Discussion}

This study has shown that for this method of broadband insonification in the geometric scattering region:

1. For the elastic-shelled animal, acoustic estimates of size using pulse compression processing were accurate for all distributions of orientation studied, in spite of the fact that the target strength varied by $10 \mathrm{~dB}$ over the same distributions.

2. For the fluid-like animal, accurate acoustic estimates of size using pulse compression processing and the simple two-ray model require that the animal's in situ orientation distribution be within about $35^{\circ}$ of broadside incidence.

3. The fluid-like animal had a change of $3 \mathrm{~dB}$ in target strength averaged over a wide frequency band for different orientation distributions.

Acoustic scattering models are vital to understanding and interpreting measurements of biological scattering in the ocean. However, these models depend upon a variety of parameters which are often unknown, such as the animal's size and orientation distribution. This study presents results that show how different animal orientations impact measurements of target strength and scattering-model-based estimates of animal size for two types of zooplankton (fluid-like and elastic-shelled).

Target strength measurements at these high frequencies show a range of values for the different orientation distributions for both types of zooplankton. Changes were smaller for the fluid-like animals, but still quite substantial (a $3 \mathrm{~dB}$ difference corresponds to a factor of two change in biomass estimates). Differences for the elastic-shelled animal were even larger.

Size estimates of single animals can be made with the use of pulse compression processing. In spite of the large variability of target strength for various orientations 
for elastic-shelled animals, the different orientation distributions caused only small changes in the acoustic estimates of size. With the ray model used, orientation information may not be needed for accurate size estimates of the elastic-shelled animals. However, for fluid-like animals, acoustic estimates of size were not accurate unless the orientations were within about $35^{\circ}$ of broadside incidence.

Target strengths and size estimates show a possibility for being used to categorize different animal behaviors. This method seems particularly suited to flud-like animals where studies have shown the strong effect that animal orientation has on scattering spectra. Since animal behavior (and the orientation distribution) will change throughout the day, more studies of animal in situ orientation are needed if acoustic scattering models are to be used correctly and effectively.

\section{Acknowledgements}

The scattering data for the shrimp was collected by Duncan E. McGehee, Timothy K. Stanton and Dezhang Chu. The snail data was collected with the assistance of Timothy K. Stanton and Dezhang Chu. These three also reviewed this chapter and suggested changes which have been incorporated. 


\section{Chapter 3}

\section{In Situ Measurements of Acoustic Target Strengths of Siphonophores, a Gas-bearing Zooplankter}

No sound is dissonant which tells of life.

- Samuel Taylor Coleridge

\subsection{Abstract}

Acoustic target strengths of free-swimming siphonophores were measured in situ at 24 and $120 \mathrm{kHz}$ from a remotely operated vehicle equipped with both acoustic transducers and a video camera. The transducers and camera were $\mathrm{co}^{-}$registered by aiming both instruments at the same volume of water and time-stamping the recorded data. The video system allowed us to search for and identify siphonophores, and verified whether individual animals were centered in, or near, the axis of the acoustic beams. A towed, down-looking acoustic survey system (operating at $120 \mathrm{kHz}$ ) measured the target and volume scattering strengths of scattering layers, presumed to be dominated by siphonophores. Spatial density of the sound scatterers were estimated from survey data. Our results confirm that free-swimming physonect siphonophores have 
relatively high acoustical target strengths caused by a gas inclusion in the pneumatophore of each animal. A relatively small number of animals can dominate the backscattering detected by acoustic surveys even though other taxa may dominate the plankton on a numerical or biomass basis. Siphonophore colonies are fragile and cannot be reliably censused with nets. Our estimates of siphonophore target strengths can improve the ability to use acoustics to quantitatively census siphonophores and other taxa possessing comparably-sized gas inclusions.

\subsection{Introduction}

Acoustic surveys can rapidly survey many types of zooplankton within a large volume of water (Medwin and Clay, 1998; Wiebe et al., 1997). Unfortunately, acoustic backscatter patterns from these surveys usually cannot be converted to quantitative estimates of the densities and identities of sound scatterers by a simple method. This is a consequence of the diverse morphologies and material properties of the organisms that make up most zooplankton assemblages (Stanton et al., 1994a).

Measurement of acoustic target strengths from different taxa is essential for development of scattering models and, ultimately, the extraction of meaningful biological parameters (for example identity, abundance, and size) from acoustic backscatter data. Well-controlled measurements of target strength are both difficult to obtain and logistically complex. For these reasons, most target strength measurements of zooplankton have been made in the laboratory (Stanton et al., 1994a, 1996, 1998a).

Although laboratory measurements can be highly controlled, they suffer from serious artifacts because the animals must be constrained in an artificial setting. The resulting measurements may be biased by low hydrostatic pressure and restricted animal orientation. Further, the mechanical and physiological stresses associated with the capture of the animal may influence target strength estimates (Stanton et al., 1998a). There is a need for well-controlled estimates of acoustic target strengths of live animals under natural conditions where animals are free to select preferred depths, water temperatures, water densities, and orientations. 
Physonect siphonophores (Figure 3-1) are important constituents of the zooplankton because of their large size and their predation upon copepods, decapod shrimp, fishes, and other taxa (Biggs, 1977; Mackie et al., 1987). Several studies report that they are widely distributed (Totton, 1965), abundant (Pugh, 1975), and potentially important sources of acoustical backscattering (Barham, 1963, 1966). These animals are colonial organisms (Gould, 1984) and for simplicity, in this paper the term "colony" will be synonymous with "animal". A single animal is made up of a pneumatophore (a gas inclusion), nectophores (tissues used for propulsion), tentacles, and gastrozooids.
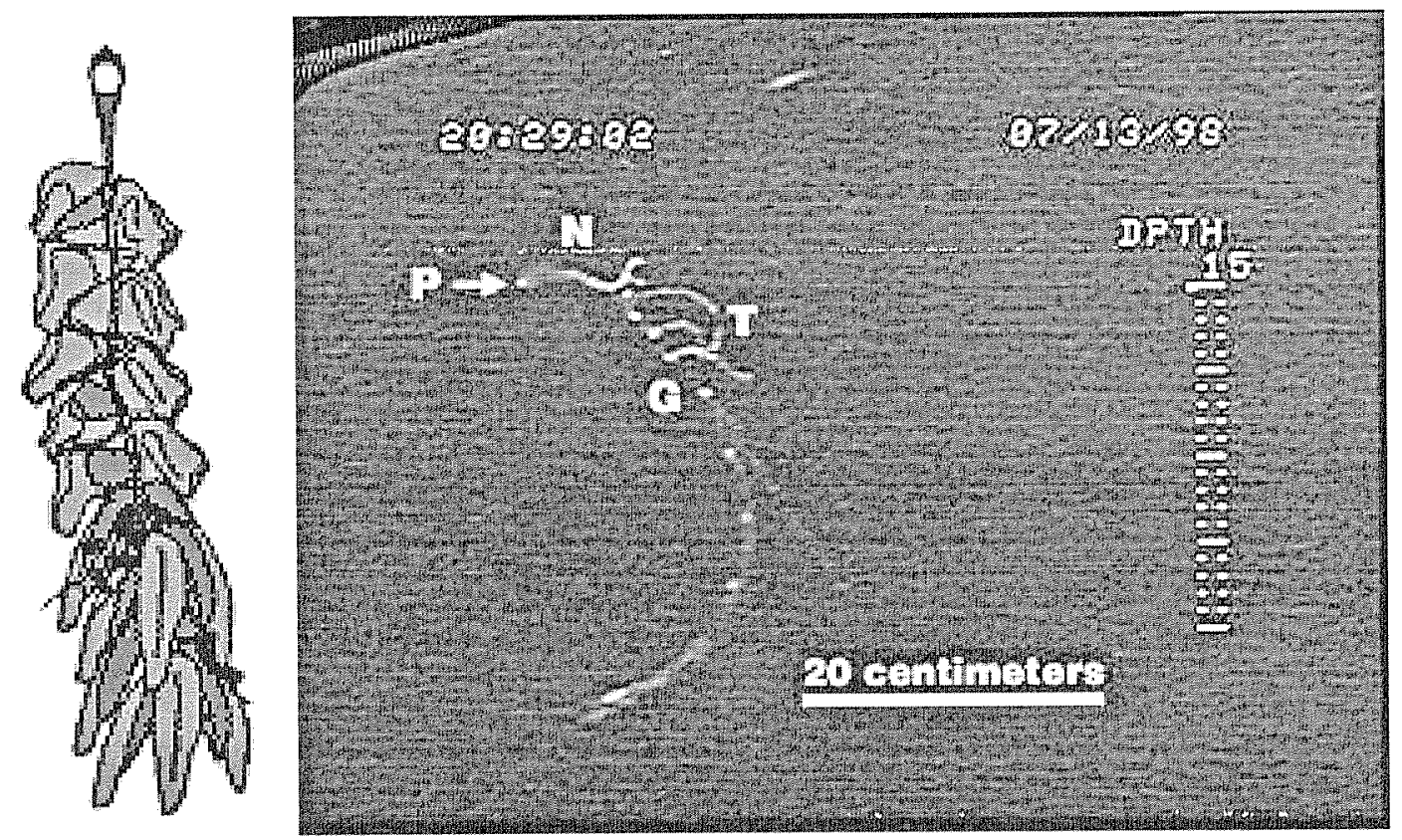

Figure 3-1: Drawing of a siphonophore (left) with a video capture of a live Nanomia cara (right). The animal consists mostly of gelatinous tissue $(\mathrm{T})$ with the exception of a gas inclusion (pneumatophore) $(\mathrm{P})$ at the top. This gas inclusion can be a significant source of acoustic scattering. The animal moves by using nectophores $(N)$ to propel itself, and has numerous gastrozooids $(G)$ with which it feeds.

Because of the extreme fragility of these organisms, most studies of siphonophores have been restricted to direct observations (Madin, 1988; Robison et al., 1998). Typical sampling equipment, such as nets and pumps, often destroy animals so in situ observations are generally required. Large siphonophores are also competent swim- 
mers (speeds of $20-30 \mathrm{~cm} \mathrm{~s}^{-1}$ have been estimated (Mackie et al., 1987)) that may be capable of evading slow-moving nets. Because of the challenges in using direct sampling methods on these animals, remote sensing methods such as acoustics provide a potential alternative. Optical methods are attractive, but the sampling volumes are usually not large enough to effectively census siphonophores (Davis et al., 1992).

A key element in the use of acoustics for assessing distribution, abundance, and identity of zooplankton assemblages is to understand the acoustic scattering properties of the scatterers. Each physonect siphonophore possesses a gas inclusion called a pneumatophore that is filled with carbon monoxide (Pickwell et al., 1964). The pneumatophore, rather than the other gelatinous colony parts, is responsible for the strong acoustic return from these animals (Stanton et al., 1998b). The size and physical properties of this gas inclusion may change with increasing hydrostatic pressure which consequently changes the acoustic scattering properties of the animal. At frequencies near resonance for the gas inclusion, target strengths may increase by more than $10 \mathrm{~dB}$ compared with those at higher frequencies (Figure 3-2).

Most recent siphonophore target strength estimates have been derived from backscattering data collected in tanks where the animals were tethered at a shallow depth $(\sim$ $1 \mathrm{~m}$ ) (Stanton et al., 1994a, 1996, 1998a), but there have been some recent estimates derived from acoustic survey data in the ocean where the presumed scatterers were siphonophores (Greene et al., 1998). While the laboratory work provided physical insights into the scattering properties of siphonophores, estimates of the acoustic target strengths of these organisms derived from such studies may not correspond directly with measurements obtained from free-ranging animals because of the constraints upon the animal in a laboratory environment (Stanton et al., 1998b).

To provide more realistic estimates of acoustic target strength of siphonophores, we collected in situ target strength measurements of the animals in the ocean. The present study utilized a remotely operated vehicle (ROV) and a towed, down-looking system to collect in situ measurements of acoustic target strengths of free-ranging physonect siphonophores at two frequencies. The results are compared with the pre- 


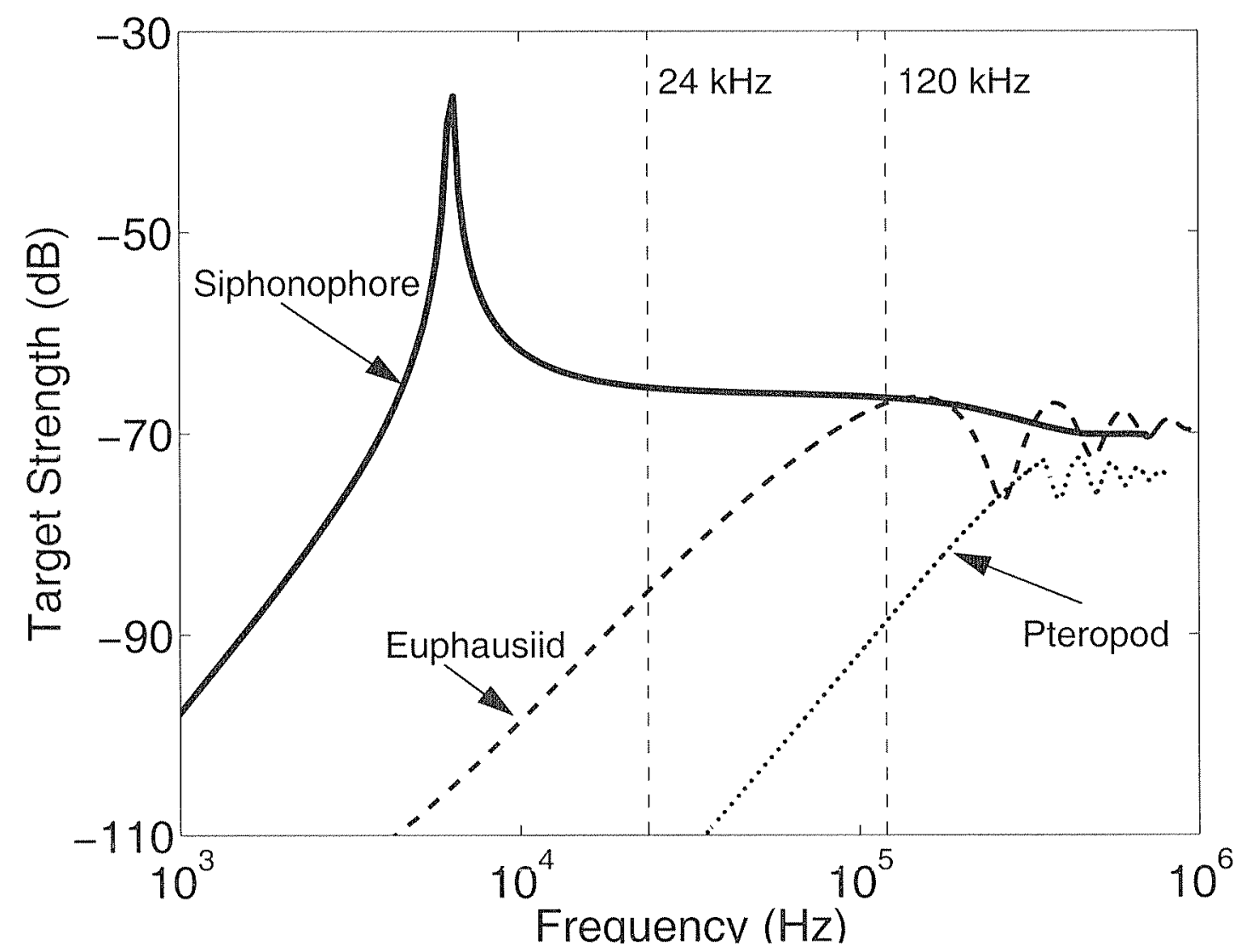

Figure 3-2: Theoretical calculations of target strength for three different types of zooplankton (Gas bearing: $1 \mathrm{~mm}$ siphonophore pneumatophore. Fluid-like: $3 \mathrm{~cm}$ long euphausiid. Elastic-shelled: 1 mm diameter pteropod.) using models from Stanton et al. (1998b). Gas bearing animals have a much higher target strength at low frequencies than the other two classes (fluid-like and elasticshelled), however at higher frequencies, the scattering levels are similar for all animal types. Dashed vertical lines indicate frequencies of 24 and $120 \mathrm{kHz}$. 
vious laboratory measurements and predictions of target strength from zooplankton from other taxanomic groups and at other frequencies.

\subsection{Methods}

This study was conducted from the R/V "Sea Diver" in the waters in and around Massachusetts Bay near Cape Cod, Massachusetts, USA, during July 1998. Two acoustic backscatter systems were used simultaneously: an ROV-mounted, multifrequency acoustic array; and a towed, down-looking echosounder. The towed system mapped spatial patterns of acoustic backscatter from aggregations of animals, while the ROV was used to measure acoustical scattering from individual animals at short ranges. A camera system was mounted on the ROV for identification of species and tracking of targets. Conductivity, Temperature and Depth (CTD) casts and Reeve net collections (Reeve, 1981) were taken at various stations during the cruise.

\subsubsection{ROV System}

We used a MaxROVER ROV (Deep-Sea Systems) operated by the National Undersea Research Program of the National Oceanic and Atmospheric Administration. The vehicle was tethered from the ship via an umbilical cable (for power supply and data telemetry) and was guided by an operator on the ship. Multiple horizontal and vertical thrusters provide the vehicle with maneuverability in three dimensions.

A three frequency $(24 \mathrm{kHz}, 120 \mathrm{kHz}$, and $200 \mathrm{kHz})$ acoustic array consisting of transmit and receive pairs of transducers (Airmar Corp.) was mounted on the front of the ROV (Figure 3-3a). The $120 \mathrm{kHz}$ and $200 \mathrm{kHz}$ transducers were aimed at the same focal point located $1 \mathrm{~m}$ in front of the array. This focal point was located near the center of the viewable area and within the depth of field of one of the video cameras. By marking the acoustic focal point on the video monitor, we were able to determine when an animal was located on the center axis of the beams of the two higher-frequency transducer pairs. Because of the broad beamwidth of the $24 \mathrm{kHz}$ 
transducers, the transducers were mounted side by side aimed in directions parallel to each other while maintaining a composite beam pattern similar to one that would be achieved if they were "focussed" at a single point. All echo data that were recorded were in the far-field (distances greater than 52, 38, and $18 \mathrm{~cm}$ ) of the 24,120 , and 200 $\mathrm{kHz}$ transducers, respectively. Unfortunately, electrical noise produced by the ROV thrusters and other systems severely degraded the quality of the $200 \mathrm{kHz}$ data and we were only able to utilize data from the 24 and $120 \mathrm{kHz}$ transducers.

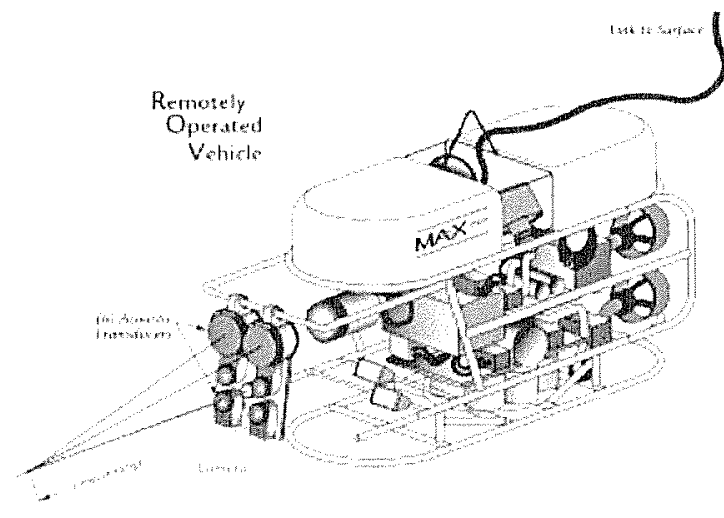

(a)

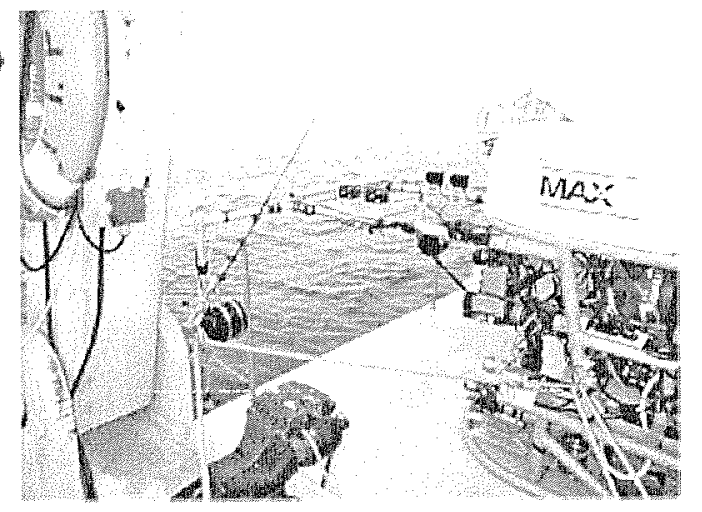

(b)

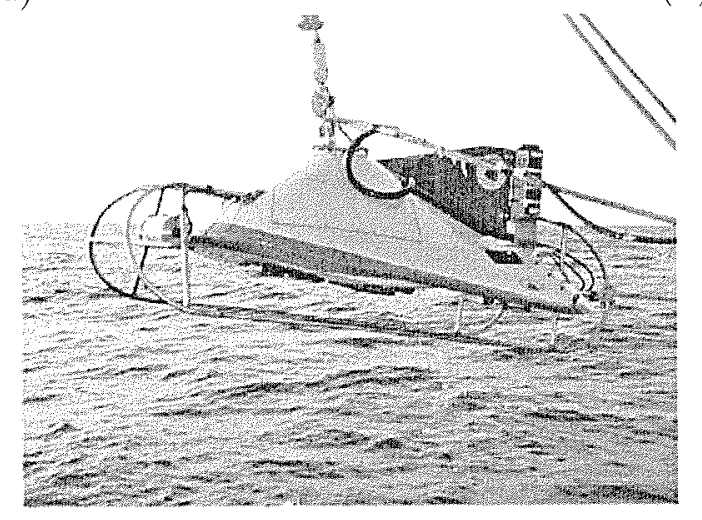

(c)

Figure 3-3: To collect in situ target strength data, the acoustic transducers were configured on the front of the ROV Kraken with a video camera all focused on the same volume for co-located acoustic and video data (a), during calibration a direct path configuration for each pair of transducers was used (each pair separated by either 0.68 or $1.73 \mathrm{~m}$ and facing each other)(b). The Greene Bomber down-looking system (c) collected echo integration data for aggregations of animals in the water column as well as target strengths of individual scatterers. 


\subsubsection{ROV Data Collection}

Collection of ROV-based echo data began when the ROV was deployed over regions of water where the echosounder had located layers of elevated backscatter. The ROV pilot then used one of two strategies to position the ROV so that the siphonophores were aligned on the acoustic axis. The ROV and associated acoustic system were always aimed into the current so that there would not be artifacts in the signal due to the wake of the ROV. One strategy was to bring the ROV close to the ship and then allow it to drift down-current with the water mass. During this period, the ROV was guided toward nearby siphonophores until they were aligned on, or near, the acoustic: axis. A second strategy was to allow animals to drift toward the ROV and then apply adjustments to the thrusters to extend the period of time that animals were along the acoustic axis. Using these techniques, we were able to track a specific animal for periods of up to 1 minute, however the maximum number of consecutive detectable echoes of an individual animal was generally five to ten with a maximum of 30 . The ping rate varied between 0.5 and $1 \mathrm{~Hz}$ and ten to fifteen runs of two or three hundred pings each were collected at a given frequency and at a given location. Echo data were collected at depths 10-30 $\mathrm{m}$ below the sea surface. This limited depth range was a consequence of the short cable length $(50 \mathrm{~m})$ which prevented signal degradation and noise problems.

Wire telemetry from the ROV enabled data to be transferred to the ship where video images and acoustic data were registered with a time-stamp and recorded onto Hi-8 video tape and a computer hard-drive, respectively. The hardware and methodologies used to record echoes matched those used for tank-based measurements of zooplankton backscatter conducted at sea and on land (Stanton et al., 1994a, 1998a), except for the addition of two hardware signal filters (Krohn-Hite Model 3200) that were needed to reduce noise from the ROV system. We collected acoustic backscatter measurements from several hundred free-ranging siphonophores over a three day period. Within the limits of resolution of the video system, the animals were identified as Nanomia cara (A. Agassiz). 


\subsubsection{ROV Calibration and Data Processing}

After collecting echo data, we arranged the transducers in a direct path configuration (Figure 3-3b) so that the transmit and receive transducers faced each other separated by a distance of $0.68 \mathrm{~m}(120$ and $200 \mathrm{kHz})$ and $1.73 \mathrm{~m}(24 \mathrm{kHz})$. Calibration data were collected in this configuration over the same depth range $(10-30 \mathrm{~m})$ that the scattering measurements were made. A calibration procedure identical to that of Stanton et al. (1998a), except for the aforementioned transducer configuration, was used.

Examination of the video and acoustic data revealed that some measurements were collected from a solitary animal while others were from two or more animals. Accordingly, only pings from single animals were used to estimate target strengths. It was not possible to hold each animal precisely along the acoustic axis because both the siphonophore and the ROV were moving. Such changes in the target's position expectedly introduced significant variability in echo level because of the acoustic beam pattern.

Raw echo voltage data was collected and examined briefly between data collection runs to verify that the acoustic measurement system was working correctly. The raw echo data was then converted to echo strength (ES) measurements where ES is a logarithmic measure of echo level convolved with the acoustic transducer beam pattern. On axis values of ES are equal to target strength (TS). Values of ES corresponding to off axis will be less than that of TS. In order to remove the effects of the beam pattern, histograms of echo amplitudes (once calibrated and adjusted for range from transducer) were deconvolved using the beam pattern of the transducer to produce estimates of the scatterer target strength (Clay, 1983; Stanton and Clay, 1986). The deconvolution method relies on the target having a constant probability of occurrence in the beam pattern. It was apparent that our tracking of siphonophores had changed their distribution in the beam pattern from a random one to one skewed towards the center of the beam. Theoretical echo amplitude distribution curves that were consistent with that of a random target location were then fit to the measured echo amplitude histograms while ignoring the "tracking" artifact. The curves were fit to 
minimize the least squares error between the experimental echo amplitude histogram and the theoretical curves. Errors were calculated for the distribution excluding the region of the tracking artifact. The deconvolution method was then applied to the theoretical probability distribution function with the least error to produce target, strength estimates. An analysis of these data using a different inverse method (Step-nowski and Moszynski, 2000) produced similar target strength estimates.

\subsubsection{Towed Echosounder Surveys}

A $120 \mathrm{kHz}$ echosounder (Hydroacoustic Technologies Inc. (HTI)) was mounted in a fiberglass V-fin tow-body nicknamed the Greene Bomber (Figure 3-3c) (Wiebe et al., 1996, 1997). The vehicle was equipped with temperature, salinity, and fluorescence sensors. Acoustic and environmental data were transferred to the vessel, processed in real time, and recorded on digital audio tape and computer hard drives.

The vehicle was towed from the starboard side of the ship at a depth of $3 \mathrm{~m}$ and at a speed of approximately 5 knots. The transducer sampled at a ping rate of $2 \mathrm{~Hz}$. Acoustic returns were echo-integrated and averaged over $30 \mathrm{~s}$ intervals. Data were displayed in real time as volume scattering strength in $0.5 \mathrm{~m}$ depth bins.

The split-beam transducer and associated HTI hardware are capable of estimating the target strengths of individual scatterers. This capability was used to independently measure target strengths within the water column, although this system had no video information to verify target identity.

\subsubsection{Net Tows}

On three occasions, net tows were conducted to sample siphonophore layers which had been observed from the ROV video system. The nets were towed horizontally for up to thirty minutes down to a depth of $20 \mathrm{~m}$, additionally vertical casts were made. A $0.785 \mathrm{~m}^{2}$ Reeve net (Reeve, 1981) with a $333 \mu \mathrm{m}$ mesh was used. Very few (less than five pneumatophores) siphonophores were captured in the 1.5 hours of tows. None of these specimens were intact. Additional animals collected in the 
net casts included pteropods, polychaetes, ctenophores, and medusae. These animals were occasionally seen in the video images from the ROV system, but were not as visually prevalent as siphonophores. The video data clearly show that siphonophores were abundant in these waters, however our net tow data indicate shortcomings of traditional net sampling methods.

\subsubsection{Acoustic Scattering Model}

The scattering model for the pneumatophore of the siphonophore comes directly from Stanton et al. (1998b) and Anderson (1950). It is presented here for a single animal with scattering contributions from the gas-inclusion only.

Target strength $(T S)$ is a logarithmic function of the scattering amplitude

$$
T S=10 \log \left|f_{b s}\right|^{2}
$$

where $f_{b s}$ is the scattering amplitude in the backscatter direction. For a siphonophore, $f_{b s}$ is generally the sum of scattering contributions from the gas inclusion $\left(f_{b u b b l e}\right)$ and from the gelatinous tissue $\left(f_{\text {tissue }}\right)$. Scattering from the tissue is quite weak (at least 10 $\mathrm{dB}$ less than scattering from the gas inclusion) and is not included in our calculations (Stanton et al., 1998a,b).

The equation used for $f_{\text {bubble }}$ is the exact solution for scattering from a fluid sphere (Anderson, 1950).

$$
f_{b u b b l e}=-\frac{i}{k_{1}} \sum_{m=0}^{\infty}(2 m+1)(-1)^{m} b_{m}^{(f)}
$$

The modal series coefficient $\left(b_{m}^{(f)}\right)$ is defined as

$$
b_{m}^{(f)}=\frac{-1}{1+i c_{m}}
$$

and

$$
c_{m}=\frac{\frac{j_{m}^{\prime}\left(k_{2} a\right) \cdot n_{m}\left(k_{1} a\right)}{j_{m}\left(k_{2} a\right) \cdot j_{m}^{\prime}\left(k_{1} a\right)}-\frac{g h n_{m}^{\prime}\left(k_{1} a\right)}{j_{m}^{\prime}\left(k_{1} a\right)}}{\frac{j_{m}^{\prime}\left(k_{2} a\right) \cdot j_{m}\left(k_{1} a\right)}{j_{m}\left(k_{2} a\right) \cdot j_{m}^{\prime}\left(k_{1} a\right)}-g h}
$$


where $j_{m}$ and $n_{m}$ are spherical Bessel and Neumann functions of the $m$ th order; $j_{m}^{\prime}, n_{m}^{\prime}$ are the derivatives with respect to $k a ; k_{1}, k_{2}$ are the acoustic wave numbers in media outside $\left(k_{1}\right)$ and inside $\left(k_{2}\right)$ the gas inclusion; $a$ is the equivalent spherical radius of the gas bubble; $g=\frac{\rho_{2}}{\rho_{1}}$ and $h=\frac{c_{2}}{c_{1}}$ are the density $(\rho)$ and sound speed $(c)$ ratios of the gas inclusion $\left(\rho_{2}, c_{2}\right)$ and its surrounding media $\left(\rho_{1}, c_{1}\right)$. When numerically evaluating the summation, the upper limit is replaced by $k_{1} a+10$ which is generally the point at which the sum converges.

\subsection{Results}

Echoes from individual siphonophores were generally strong and of high quality (Figure 3-4), but varied (Figure 3-5). Variations in echo strength were associated with changes in the position of the animal in relation to the focal point of the beam. Since multiple animals were studied, the size of the pneumatophore is expected to vary, which would also cause variability in echo levels. Mean target strengths calculated by the deconvolution method applied to echoes collected by the ROV system were $-59.9 \mathrm{~dB}$ at $24 \mathrm{kHz}$ and $-69.1 \mathrm{~dB}$ at $120 \mathrm{kHz}$. The mean echo strength, which is a logarithmic measure of the echo amplitude convolved with the beam pattern (resulting in an underestimate of target strength) of individual siphonophore animals was found to be $-62.5 \mathrm{~dB}$ at $24 \mathrm{kHz}$ and $-70.1 \mathrm{~dB}$ at $120 \mathrm{kHz}$ (Table 3.1). Echo strength is equal to target strength when the animal is aligned with the acoustic axis, but when the animal is located off-axis then the echo strength is smaller due to beam pattern effects (less acoustic energy insonifies the target when it is off-axis). Target strength measurements assume that the animal is located exactly in the center of the acoustic beam so the maximum amount of acoustic energy is incident on the target. The echo strength measurements were based on data when siphonophores were located near, but not exactly at, the center of the video image.

Both the ROV-mounted array and the down-looking system produced comparable estimates of the target strengths. While we could not identify the targets measured 


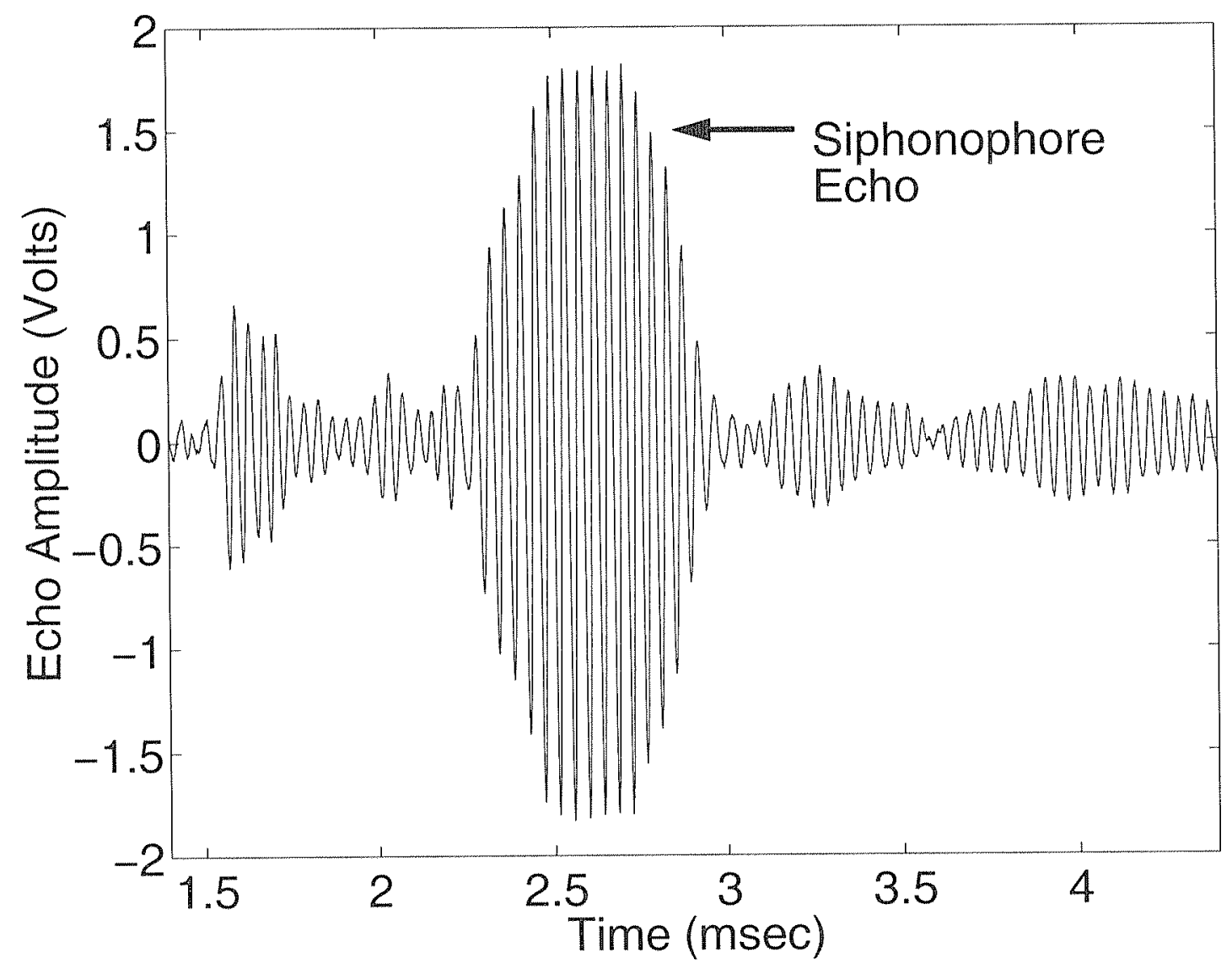

Figure 3-4: An example of the high quality echo that could be detected with the acoustic system mounted on the ROV. This $24 \mathrm{kHz}$ echo from a single siphonophore is quite similar to the transmitted signal. 

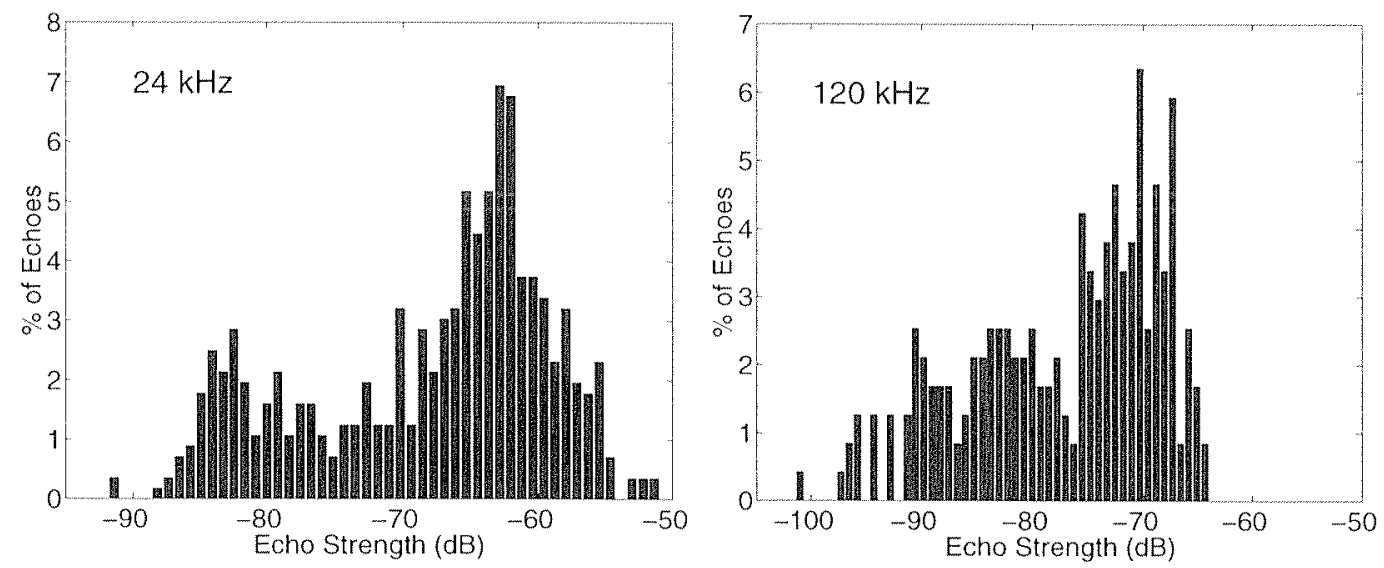

Figure 3-5: Echo strength histograms for 24 and $120 \mathrm{kHz}$ from the ROV-based system. Echo strength is a logarithmic measure of echo level convolved with the beam pattern. It is possible that the peak in the left tail of this histogram is the result of scattering from the tissue of the animal when the bubble moved out of the beam of the acoustic transducer. A simple calculation based upon a model developed by Stanton et al. (1994a) estimates that the scattering from siphonophore tissue as -80.3 $\mathrm{dB}$ at $24 \mathrm{kHz}$ which agrees with the peak in the left tail of the $24 \mathrm{kHz}$ histogram.

Table 3.1: Mean echo strength ( $\overline{E S})$ and target strength $(\overline{T S})$ data from ROV based measurements of siphonophore echoes for various frequencies and subsets of data. The echo strength is a logarithmic measure of echo level convolved with the beam pattern. On-axis ES values correspond to TS; off-axis values of $\mathrm{ES}$ are smaller than TS due to beam pattern effects (less acoustic energy insonifies the target when it is located off-axis). Target strength measurements assume that the animal is located directly in the center of the acoustic beam. Large Returns are those echoes $>-76 \mathrm{~dB}$. Measurements of the three echoes when the pneumatophore was visually confirmed to be exactly centered in the acoustic beam pattern have no beam pattern effects and are target strength measurements. The similarity in the $24 \mathrm{kHz}$ data support the assumption that the gas inclusion in these animals is the dominant scattering mechanism.

\begin{tabular}{|c|c|l|l|l|}
\hline $\mathrm{f}(\mathrm{kHz})$ & \# of Echoes & Data Set & ES $(\mathrm{dB})$ & $\overline{T S}(\mathrm{~dB})$ \\
\hline 24 & 561 & All & -64.5 & \\
24 & 431 & Large Returns & -62.5 & \\
24 & 3 & Pneumatophore & & -62.5 \\
120 & 236 & All & -73.9 & \\
120 & 130 & Large Returns & -70.1 & \\
\hline
\end{tabular}


by the down-looking system, histograms of target strengths from the $10-20 \mathrm{~m}$ layer (Figure 3-6) are similar to the histogram derived from the $120 \mathrm{kHz}$ in situ array which collected data predominantly from 20-30 $\mathrm{m}$ depth. The cause of the increase in target strength with depth shown in the down-looking system data is not known.
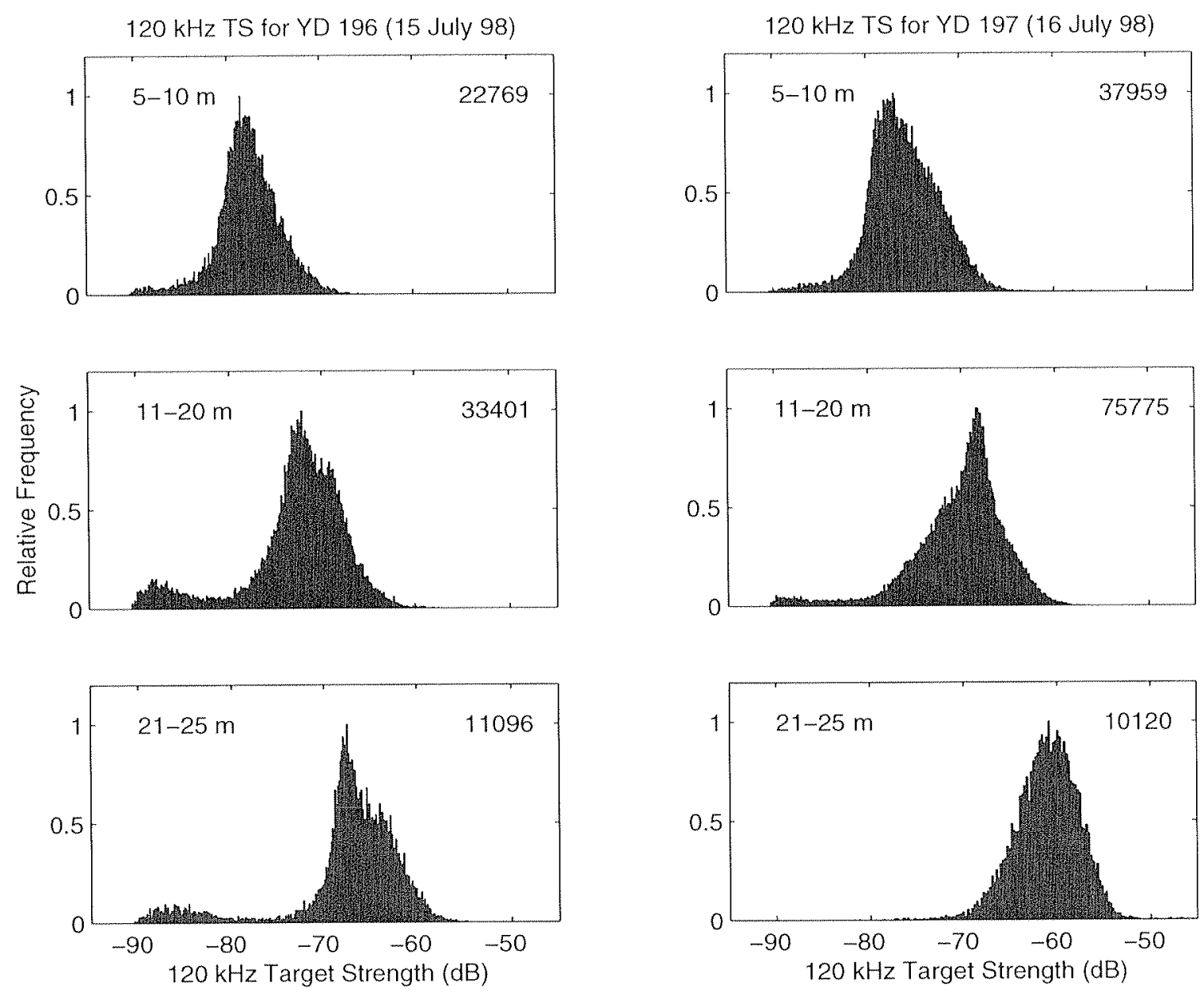

Figure 3-6: Target strength histograms from the Greene Bomber down-looking $120 \mathrm{kHz}$ system for three different depth ranges of targets. The Greene Bomber was towed at a depth of $3 \mathrm{~m}$. The number of individual target strengths making up a histogram is given in the upper right corner of each figure.

Our down-looking acoustic surveys revealed the presence of strong scattering layers between 0-30 $\mathrm{m}$ (Figure 3-7). These layers varied in depth and time and there was a regular diel migration coinciding with sumrise and sunset. Examination of the video images from the ROV collected while the down-looking system was in oper- 
ation suggests that siphonophores were the most significant sound scatterers in the water column. Smaller animals, not seen in the video images, also may have been numerous, but would not be detected by the frequencies used in the acoustic systems due to their small size resulting in very weak scattering at 24 and $120 \mathrm{kHz}$. Solitary euphausiids were observed with the video system, although they appeared to avoid the ROV. Other zooplankton present in either the video or Reeve net samples included copepods, pteropods, polychaetes, ctenophores and an occasional medusae. The scattering that was detected by the ROV-mounted array when targets other than siphonophores were in the field of view was extremely weak (generally less than -80 $\mathrm{dB})$.

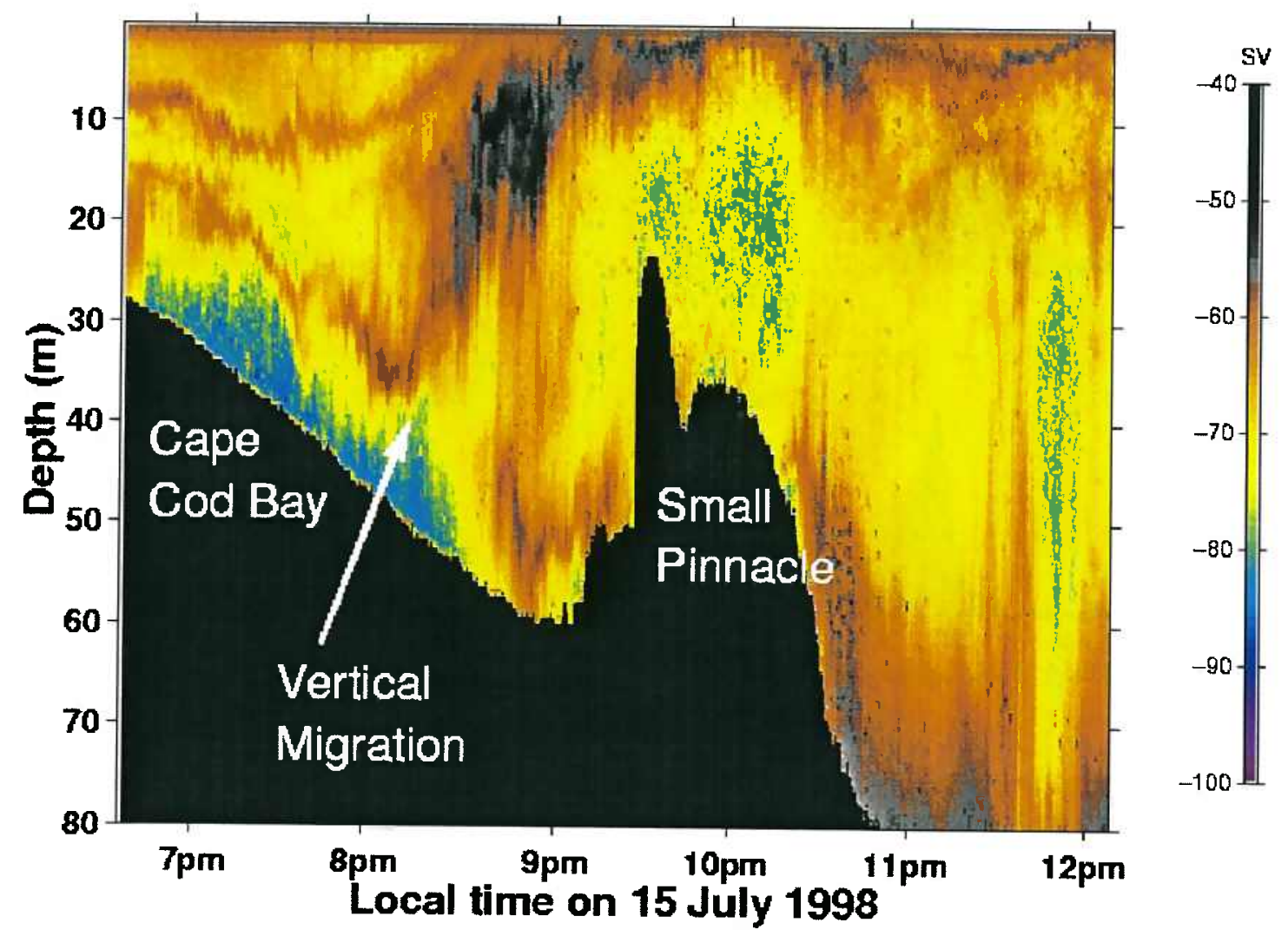

Figure 3-7: Pattern of volume backscattering strength $\left(S_{v}\right)$ in the water column during an acoustic survey transect with the down-looking system. Strong scattering regions include a vertically migrating patch occurring at sunset and patches located at the water surface. The transect was in a straight line from Cape Cod Bay to Stellwagen Bank at a constant speed, so the time axis is directly proportional to distance. 
Volume scattering strength of the water column varied by several orders of magnitude (Figure 3-7) with a peak value of $-57 \mathrm{~dB}$ in the vertically migrating layer although scattering from surface patches at night were also strong. Mean siphonophore target strength at $120 \mathrm{kHz}$ was estimated to be $-69.1 \mathrm{~dB}$ from 236 echoes from the ROVbased measurements. Animal abundance density $\left(n_{b}\right.$, with units of \# of animals $\mathrm{m}^{-3}$ ) could then be estimated by

$$
n_{b}=\frac{s_{v}}{\left\langle\sigma_{b s}\right\rangle}
$$

from Medwin and Clay (1998) where $s_{v}$ is the volume backscattering coefficient derived from the down-looking system echo integration data and $\left\langle\sigma_{b s}\right\rangle$ is the average differential backscattering cross section from the ROV measurements $\left(\sigma_{b s}=\left|f_{b s}\right|^{2}\right)$. Density estimates based on Eq. 3.5, ROV-based target strength measurements (Table 3.1), and down-looking system volume scattering strength data (Figure 3-7) range from 1 to 3 siphonophores $\mathrm{m}^{-3}$ for regions where the ROV measurements occurred and a peak density of 15-20 siphonophores $\mathrm{m}^{-3}$ in the vertically migrating patch and the near surface layers. Visual observations from the ROV video system qualitatively agree with the lower density estimate. These observations are consistent with density estimates ( $1-7$ animals $\mathrm{m}^{-3}$ ) made in the Gulf of Maine from a submersible (Rogers et al., 1978). If target strength estimates from the down-looking system are used, density estimates rise by an order of magnitude. Since the ROV-based measurements include visual identification of the scatterer, those target strength and numerical density predictions are believed to be more accurate.

\subsection{Discussion}

The use of backscattered acoustical energy as a method to estimate biomass and animal distribution is quite common. The interpretation of acoustic data is still a challenging endeavor. Acoustic waves are reflected from variations in the density and sound speed of the medium that the wave travels through (either the water column or the animal's body). Theoretical models used to describe the scattering characteristics 
of zooplankton have undergone a tremendous evolution in the last twenty years (see review in Foote and Stanton (2000)) and these model predictions agree well with laboratory data. It is well documented that scattering levels from gas bubbles increase as the acoustic frequency approaches the resonance frequency (Anderson, 1950). Since $24 \mathrm{kHz}$ was near the resonance frequency of the gas inclusion, the system operating at that frequency yielded the highest target strength. This frequency, or ones near it, may be useful for quantifying the distributions and abundances of organisms containing gas inclusions such as siphonophores and small fishes, since target strengths of other zooplankton, such as fluid and shelled animals, are negligible near these frequencies, while at higher frequencies the target strengths become more similar (Figure $3-2$ and Table 3.2 ).

Table 3.2: Target strength predictions at $24 \mathrm{kHz}$ and $120 \mathrm{kHz}$ for siphonophores compared to other animals using models from Stanton et al. (1998b). The $1 \mathrm{~mm}$ size of the siphonophore is for the gas bubble only. Differences are much smaller at the higher (and more commonly used in acoustic survey applications) frequency, while quite substantial at the lower frequency. These predictions (supported by measurements in this paper) suggest that lower frequencies may be useful for surveying siphonophores since the target strengths of other zooplankton are negligible when compared with those of siphonophores.

\begin{tabular}{|l|l|c|c|}
\hline Animal & Size $(\mathrm{mm})$ & TS $(\mathrm{dB})$ at $24 \mathrm{kHz}$ & $\mathrm{TS}(\mathrm{dB})$ at $120 \mathrm{kHz}$ \\
\hline Shrimp & 30 (length) & -86 & -67 \\
Pteropod & 1 (diam.) & -116 & -89 \\
Siphonophore & 1 (diam.) & -66 & -66 \\
\hline
\end{tabular}

A distinct advantage of laboratory studies has been that an animal can be positioned in the center of the beam pattern of the acoustic transducers. Under natural conditions, the animal's location within the acoustic beam is variable. Deconvolution methods (Clay, 1983; Stanton and Clay, 1986) have been applied to acoustic studies of various fish schools. These methods use echo statistics and the characteristics of the acoustic transducer to remove beam pattern effects from echo data involving resolved targets. Our data are generally suited for this type of analysis, although not optimally due to the "tracking" of animals by the ROV pilot. Instead of collecting data from a uniform distribution of targets in the beam pattern, our data are skewed towards the center of the beam pattern creating an upward bias in the results which we removed 
by visually fitting the data to theoretical echo amplitude histograms for a randomly located target. Although the "tracking" of the animals introduced this artifact, it was necessary in order to collect enough echoes to fully resolve the distribution of target strengths.

Scattering levels of individual siphonophores measured at depth were consistent with scattering from a gas inclusion with a diameter of about $1 \mathrm{~mm}$, a dimension similar to pneumatophore diameter measurements made at the surface from several animals captured by net tows. The in situ target strength estimates are generally consistent with previous studies (Figure 3-8). Greene et al. (1998) estimated the target strength of siphonophores to be $-75 \mathrm{~dB}$ at $420 \mathrm{kHz}$ from measurements made in situ with an echosounder attached to a net sampling system. The target strength estimate was determined by examining the scattering from a region where the net sample was dominated by siphonophores but also contained other animals which would scatter acoustic energy. Measurements from individual siphonophores made at $120 \mathrm{kHz}$ by Stanton et al. (1994a) were slightly higher than what is presented here. While there is much variability in these estimates, the results from this study are in situ measurements from siphonophores and are probably more accurate than previous estimates from field or laboratory data.

The data from the down-looking system show an increase in target strength with increasing depth (Figure 3-6). One possible explanation is the greater likelihood of multiple targets being recognized incorrectly as single targets by the split-beam system. Another possible explanation is that increasing pressure may cause pneumatophore size to decrease which would cause an increase in target strength near the resonance frequency of the bubble (Figure 3-2). It must be emphasized that this latter explanation is possible but perhaps not likely, since siphonophores are able to regulate the volume of gas in the pneumatophore (Mackie et al., 1987).

Our results are important for two reasons. First, in situ measurements are consistent, with laboratory measurements and theoretical predictions of the relatively high target strengths of siphonophores. Second, the data suggest an acoustic methodol- 


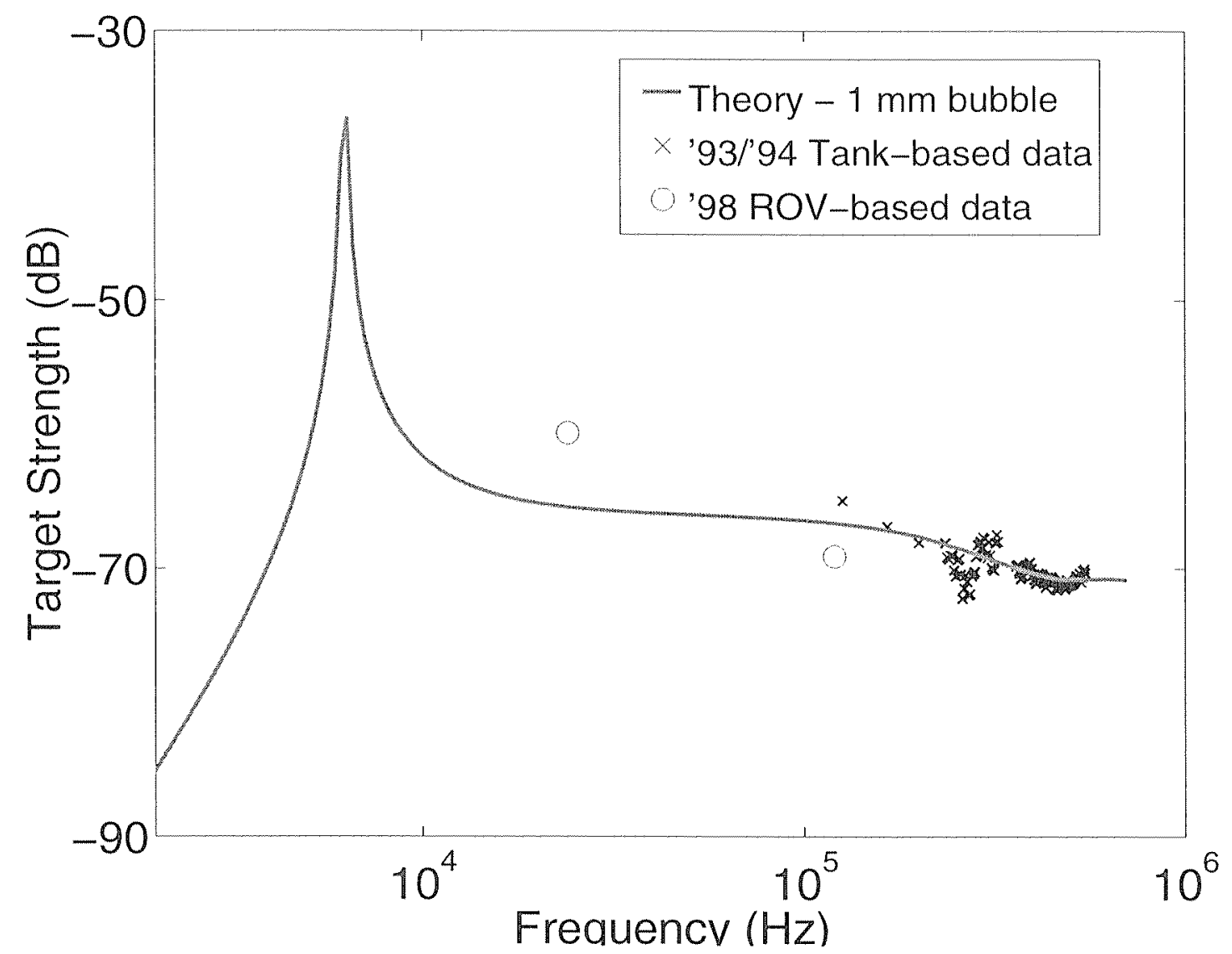

Figure 3-8: Measurements of average siphonophore target strength (o from this study, $x$ from Stanton et al. (1998b)) plotted alongside theoretical prediction for backscattering from a $1 \mathrm{~mm}$ diameter gas inclusion using Eq. 3.1 with the following values $(g=0.0012, h=0.22)$. 
ogy for quantifying abundances of siphonophores. Lower frequencies (at or near 24 $\mathrm{kHz}$ ) are desirable for censusing siphonophores and other gas-bearing animals since scattering by other types of zooplankton at this frequency are often negligible. At higher frequencies, siphonophore target strengths are comparable to those from fluidlike and elastic-shelled animals (Stanton et al., 1998b). Given the fragile nature of these organisms, acoustics may represent one of the only viable non-optic methods for quantifying siphonophore abundance.

\section{Acknowledgements}

The ROV support group (Paul Donaldson, Nick Worobey, and Matt Wetmore) were instrumental in the collection of our in situ data. Malinda Sutor directed CTD operations and assisted with the ROV. Clarence Clay (University of Wisconsin, Madison, Wisconsin) and Marek Moszyński (Technical University of Gdańsk, Gdańsk, Poland) provided insight and analysis on the beam pattern deconvolution problem. This research was funded by grant NAGL-98-01A and NAGL-98-01B from the National Undersea Research Center, North Atlantic and Great Lakes, which is a component of NOAA's National Undersea Research Program. Additional support came from the Office of Naval Research Grants N00014-96-1-0878 and N00014-95-1-0287, the WHOI Education Office and the State of Louisiana. Timothy K. Stanton, Mark C. Benfield, Peter H. Wiebe, D. Chu and M. Sutor all reviewed the manuscript and offered suggestions for its improvement. 


\section{Chapter 4}

\section{Biological and Physical Sources of Scattering in an Internal Wave}

Our knowledge is a little island in a great ocean of nonknowledge.

- Isaac Bashevis Singer

\subsection{Introduction}

Acoustic methods offer biologists a useful tool in estimating the distribution and abundance of many types of marine life. Many marine animals (predominantly fish and zooplankton) will scatter a measurable amount of backscattered energy. Surveys of the water column can provide acoustic data at sub-meter resolution over several hundred meters of the upper water column and cover large horizontal distances (primarily limited by the towing speed of the ship). These methods can cover an enormous area and provide finer resolution than traditional methods of surveying biomass such as net tows. However, acoustical surveys provide information on the amount of scattered sound in the water column, not the number of biological organisms present. The interpretation of backscattered sound energy to biomass is a complex process where simple assumptions can lead to enormous errors.

There are ecological and economical reasons that the abundance and distribution 
of marine life in the oceans is important. Additionally, the Navy is interested in discriminating between scattering from natural and man-made sources. Zooplankton are one of the key elements in the marine food chain, and there is much not known about their distribution, abundance and behavior. In addition to their ecological importance, some zooplankton such as krill have emerged as a commercially important fishery (Croll and Tershy, 1998). Zooplankton distributions are also useful information for fisheries management agencies who attempt to regulate commercial fishing operations. Many fish species feed primarily on zooplankton, and thus the zooplankton population will affect the fish populations.

Many studies in the past fifty years have discussed how zooplankton and fish scatter acoustic energy (for example: Dietz (1948); Hersey and Backus (1954); Barham (1963); Castile (1975); Sameoto (1980); Stanton et al. (1994a); Holliday and Pieper (1995)) and there have also been numerous studies discussing how to convert the backscattered energy into estimates of biomass (Holliday et al., 1989; Wiebe et al., 1996; Brierley et al., 1998). Scattering from these animals depends upon the acoustic frequency, animal size, animal shape, animal behavior, and the physical properties of the animal. In addition, there are other processes in the ocean that also scatter detectable amounts of sound. The sea floor, sea surface, suspended sediments, bubbles and even the water column itself can scatter sound energy and cause difficulty in estimating biomass from acoustic data.

\section{Inverse Problem}

For an idealized case where the scattering is due to $\mathrm{N}$ identical scatterers, the volume backscattered energy, $s_{v}$, is given by the incoherent sum of the echo from each scatterer:

$$
s_{v}=\mathrm{N} \sigma_{b s}
$$

where $\sigma_{b s}$ is the differential backscattering cross-section of a scatterer and effects due to multiple scattering and extinction are ignored. If $\sigma_{b s}$ is known, then the measured scattering can be used to find $\mathrm{N}$, the number of animals in this region. This is 
the simplest form of the Inverse Problem (IP), where one inverts acoustic scattering data to find information about the scattering processes (such as the abundance of scatterers). In most ocean environments, the IP cannot be performed without many simplifying assumptions. These assumptions are used because different animals have different scattering properties depending upon the aforementioned categories, many of which are variable within and between different zooplankton species. If one attempts to solve the complete IP, then the equation becomes

$$
\sum_{i=1}^{m} s_{v}^{i}(x, y, z)=\sum_{j=1}^{n p} \sigma_{i j}(x, y, z) N_{j}(x, y, z)
$$

or in full matrix form

$$
\left[\begin{array}{c}
s_{v}^{1} \\
\vdots \\
s_{v}^{m}
\end{array}\right]=\left[\begin{array}{ccc}
\sigma_{11} & \ldots & \sigma_{1 n p} \\
\vdots & \ddots & \vdots \\
\sigma_{m 1} & \ldots & \sigma_{m n p}
\end{array}\right]\left[\begin{array}{c}
N_{1} \\
\vdots \\
N_{n p}
\end{array}\right]
$$

where $\mathrm{m}=$ the number of acoustic frequencies being used, $\mathrm{n}=$ the number of processes (typically different animal taxa) with independent scattering functions, $\mathrm{p}=$ the number of size classes for each animal, and $(x, y, z)$ refer to a volume at some 3dimensional location. Inversion of this matrix is immensely complex and may result in non-realistic, but mathematically valid, solutions. In order to attempt to solve the IP for the number of zooplankton in a given region, the Forward Problem (FP) must be well-posed.

\section{Forward Problem}

The Forward Problem is directly related to the Inverse Problem, except that the inputs of $\sigma_{b s}$ and $N_{j}$ are known, and the output, $s_{v}$, is unknown. In a field experiment, mathematical scattering models provide the values of $\sigma_{b s}$, net tows provide the abundance information $\left(N_{j}\right)$, and acoustic echosounders measure $s_{v}$ at a specific location (latitude, longitude, depth) in the ocean. This method requires that the net tow and 
echo sounder data are collected simultaneously and co-located. Adjustments to the scattering models used for the different animal types and sizes can be made to reduce errors between predicted and measured values of $s_{v}$. It is in this "diagnostic" mode that the FP will be used in this study. It was mentioned previously that both the IP and FP require that the scattering function for different processes to be known. In previous studies, the different processes were different animal taxa or size classes of a taxa. However, scattering from the water column (from temperature and salinity microstructure) is believed to be another important process and is included in this analysis.

Another critical factor in both the Forward and Inverse Problems is the number of different scatterer types present in a particular region. Jellyfish, aragonite-shelled gastropods, and shrimp are three commonly found zooplankton with little similar in their scattering physics. Different animal types have vastly different morphologies and body compositions. These physical differences lead to different boundary conditions when the acoustic scattering problem is solved for the animal, and thus different animal taxonomic types have widely varying scattering functions. Because of this, it is vitally important to know which animals are actually present in the water column. Net tows or other direct sampling methods are required to properly interpret acoustic scattering data.

However, capture of animals is time consuming (both in performing at sea and, especially so, in the enumeration and identification of samples) so a means to determine the different scattering processes occurring in the water column is needed. Theoretical studies have shown that different scattering processes have different scattering spectra. In fact, for echoes from individual animals, the scattering spectra have been used to identify the zooplankton among three different taxa (Martin-Traykovski, 1998). Also, two frequency echo-sounding systems have used differences in $s_{v}$ at the two frequencies to discriminate between schools of fish and patches of krill (Miyashita and Aoki, 1999; Brierley et al., 1998; David et al., 1999). The differences in $s_{y}$ are the result of the two animal types having different scattering spectra. These studies 
suggest that a multiple frequency acoustic system could provide information suited to differentiating between types of scatterers in a field survey.

In addition to different animals, there are other scattering processes in the ocean which have distinct spectra. Scattering from temperature and salinity microstructure in the water column is a potential contributor to the scattering measured by acoustic surveys, however these contributions will probably only be significant in regions with high levels of turbulence (Woods, 1977). Internal waves may provide the processes needed to cause detectable amounts of scattering from temperature and salinity microstructure in the water column.

Internal waves are a common feature throughout the oceans and transport a large amount of energy to coastal regions. These waves propagate along density gradients and can have amplitudes of several hundred meters, in addition to playing a role in the aggregation or transportation of zooplankton (Lennert-Cody and Franks, 1999). High values of turbulence are also associated with these features (Sandstrom et al., 1989; Hebert et al., 1992). Numerous studies have detected these features acoustically (Haury et al., 1983; Sandstrom et al., 1989; Trevorrow and Teichrob, 1994; Trevorrow, 1998), however none have been able to determine whether they were measuring acoustic scattering from the wave or from biological organisms "surfing" the wave. In order to test the ability to discriminate between biological and physical scattering, both processes must be present in the water column. Fortunately our study region, the waters of the Gulf of Maine and Georges Bank, satisfy this requirement.

\subsection{Methods}

During a GLOBEC (GLOBal ocean ECosystems dynamics) process cruise studying the populations of Calanus in the deep basins of the Gulf of Maine, an acoustic survey was undertaken to "hunt" for internal waves in the eastern part of Wilkinson Basin (located between Georges Bank and Stellwagen Bank). Internal waves are regularly generated by tidal forces pushing water on top of Georges Bank which then spills off the Bank and generates internal waves. Three survey transects were made from the 
R/V Endeavor on a series of internal waves on 16 October 1997.

\subsubsection{Acoustics}

To provide spectral information about the scattering processes occurring in the water column, acoustic scattering data were collected by the BIOMAPER-II (BIo-Optical Multi-frequency Acoustical and Physical Environmental Recorder) instrument. Before the first acoustic transect, CTD (Conductivity, Temperature and Depth) cast \# 10 was conducted. Additionally, immediately after the third transect was completed, MOCNESS (Multiple Opening and Closing Net and Environmental Sensing System) tow \# 9 was performed. These three data sets provide enough information to analyze the contributions from biological and physical sources of acoustic scattering.

BIOMAPER-II is a towed body with numerous acoustic, envirommental, video and optical sensors (Wiebe et al., submitted). The acoustic system consists of five pairs of transducers (operating at 43,120,200,420, and $1000 \mathrm{kHz}$ ), with one of each frequency looking upward and the other downward. The instrument is "tow-yo-ed" through the water column to depths within ten to twenty meters of the sea floor. The transducers have depth ranges of $200,200,150,100$, and 35 meters respectively with a depth bin size of one meter. The ping rate is variable and is normally set so that echo integration data are provided every 12 seconds. Since the instrument has a depth sensor, the upward and downward looking echo integration measurements can be merged into one data set which provides almost complete coverage of the upper water column (Figure 4-1).

The acoustic data are acquired and stored with a time stamp in the manufacturer's (HTI, Seattle, WA) format. The data files are processed and combined (using the time stamp) with data from the ESS (Environmental Sensing System) sensors which are also on board BIOMAPER-II. The upward and downward data are merged for each acoustic frequency and the final data file provides echo integrated volume scattering strength $\left(S_{v}\right)$ for the water column along with position (latitude, longitude, instrument depth), temperature, salinity, fluorescence, turbidity, and other sensor 


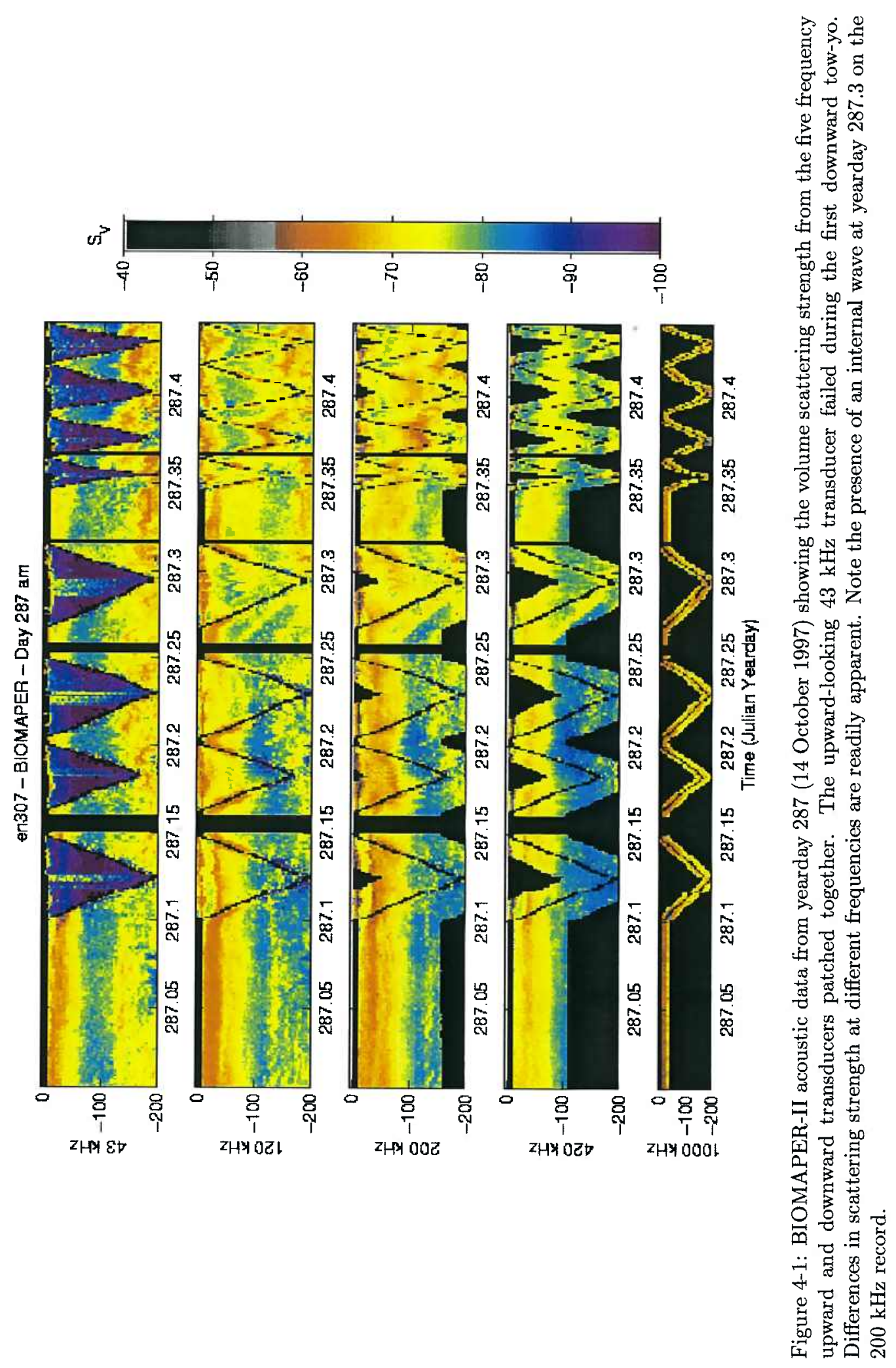


data.

Depending upon the acoustic frequency used, different regions of the water column show different scattering strengths. The data from the $1 \mathrm{MHz}$ transducers covers only 35 meters in depth and due to this limited range, are not included in any of the following analysis. The data presented here are from regions when BIOMAPER-II was towed near the surface, in regions where tow-yos were performed the $1 \mathrm{MHz}$ data would be included to further characterize the scattering in the water column.

The first transect through the internal wave shows two separate layers which have equivalent values of volume scattering strength $\left(S_{v}\right)$ at $200 \mathrm{kHz}$. However, at lower frequencies (43 and $120 \mathrm{kHz}$ ) the upper layer shows higher scattering (Figure 4-2), while at the higher frequency $(420 \mathrm{kHz})$ the lower layer has a larger $S_{v}$. The visual cues from this data series were quantified by measuring the actual $S_{v}$ values along the upper and lower portions of the wave (Figure 4-3). When comparing the measured scattering spectra for the internal wave layers to theoretical spectra for microstructure and biology (Figure 4-4); there are definite similarities. While this is not proof that the upper layer is caused by microstructure and the lower layer is caused by biology, it does suggest that distinct processes with different scattering spectra are responsible for the scattering occurring in the two layers.

A method to quantify the shape of the scattering strength spectrum is needed, so for a specific latitude, longitude and depth the scattering strength was plotted versus frequency. Two geometric shapes, a first-and second-order polynomial (straight line and parabola respectively), were fit to these data points. The parameters of these polynomial fits (slope for the first-order polynomial, curvature and concavity for the second-order polynomial) were then calculated. These parameters distinguish various regions in the water column which match those identified from a visual inspection of the four frequency curtain plots. For simplicity, these parameters will be referred to as "slope" data in the rest of this chapter. These slope measurements are not to be interpreted as describing the physics of the scattering processes, rather they are simply a parameter used to differentiate scattering spectra. 


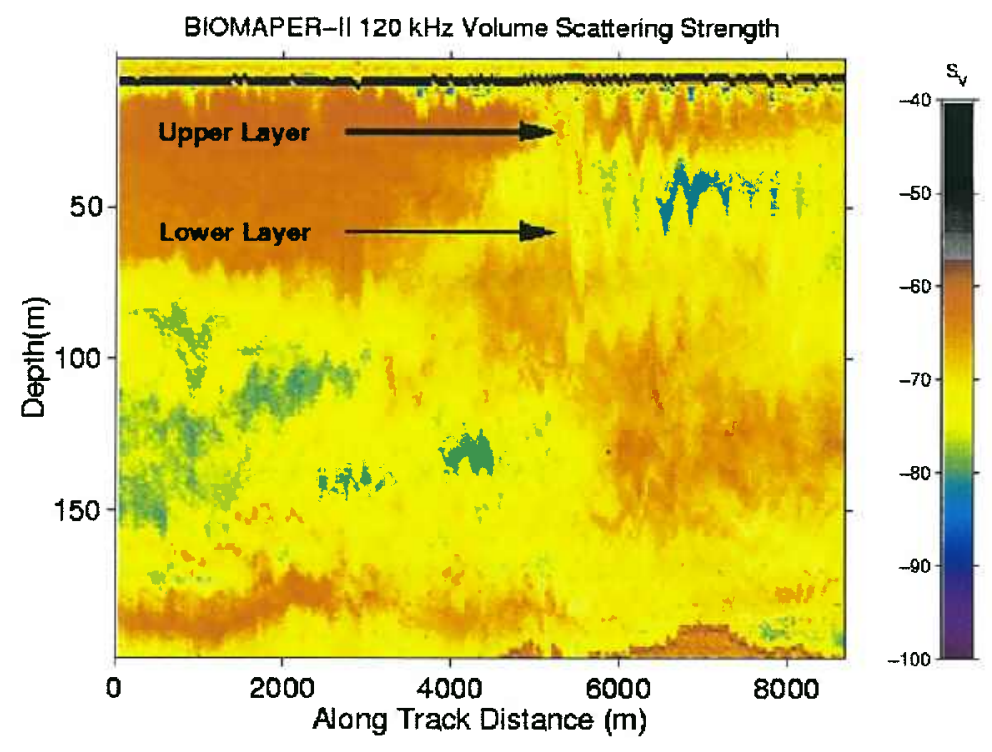

Figure 4-2: BIOMAPER-II $120 \mathrm{kHz}$ echogram of the first transect through the internal wave. The upper and lower layers of the wave are clearly seen and data collected along each wave layer is shown in Figure 4-3.

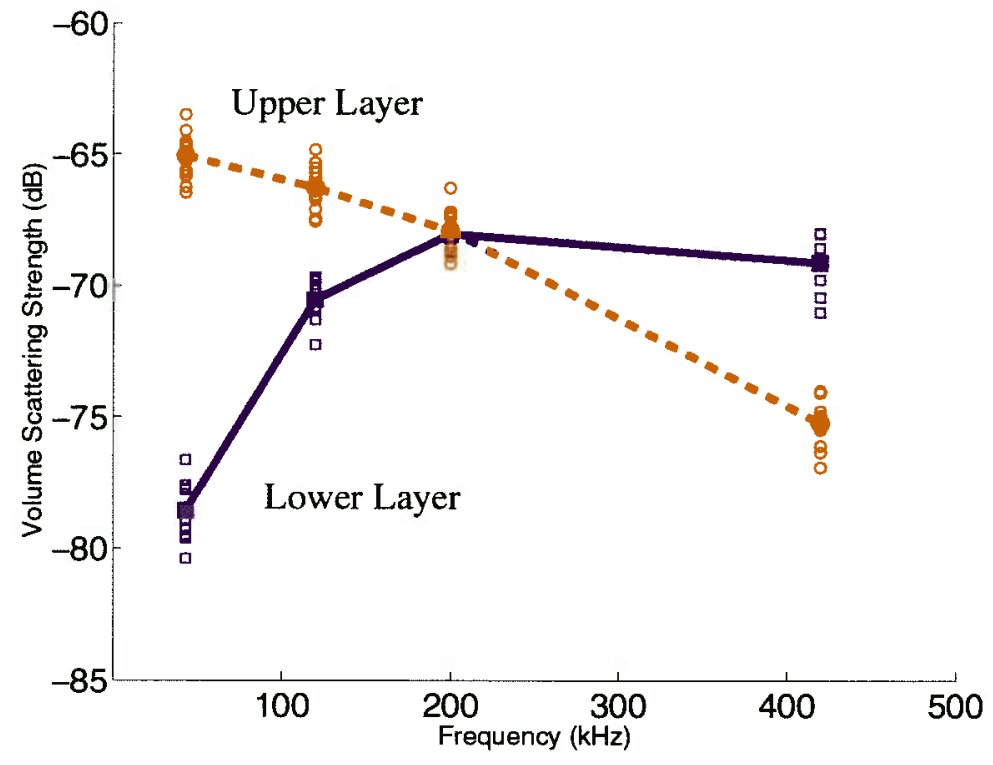

Figure 4-3: Measured values of $S_{v}$ at four acoustic frequencies from the upper and lower layers of an internal wave. The upper layer (dashed line) has a scattering spectra which decreases with frequency, while the lower layer (solid line) has an increasing scattering spectra. Dots represent $S_{v}$ values at points along each layer, while the line is through the mean values at each frequency for each layer. 


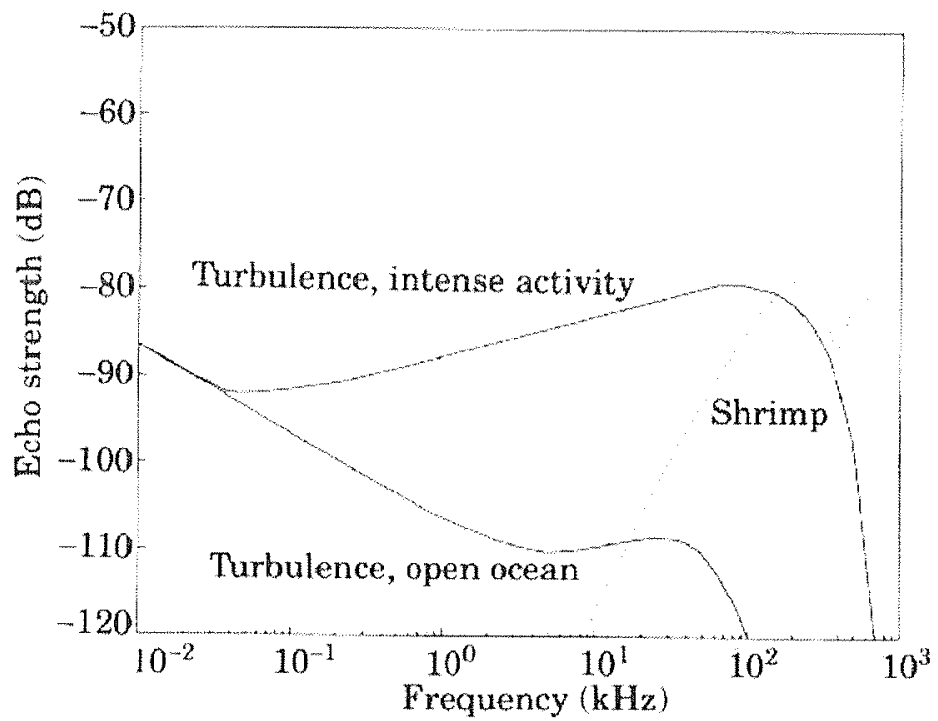

Figure 4-4: Theoretical predictions of echo strength versus frequency for microstructure and a $2 \mathrm{~cm}$ long shrimp. Turbulent microstructure have a generally decreasing echo strength with increasing frequency, while the shrimp has an increasing echo strength with respect to frequency. Figure is from Stanton et al. (1994b)

There are several difficulties with using the slope data, the foremost is that if four frequencies are used to define the slope parameters, then the data are limited to the depth range of the $420 \mathrm{kHz}$ transducer which is 100 meters. Therefore a second set of slope data was calculated where only three frequencies $(43,120$, and $200 \mathrm{kHz})$ were used to calculate the slope, so there would be 50 meters additional coverage of the water column. While there are differences in the absolute values of the slope parameters and the extent of the regions that are delineated, both the three and four frequency methods provide similar information.

\subsubsection{Zooplankton}

\section{Net Tows}

Nine $1 \mathrm{~m}^{2}$ MOCNESS (Wiebe et al., 1985) tows were collected during the cruise, with MOC 9 conducted immediately after the third acoustic transect (Table 4.1). A MOCNESS system consists of a series of nine nets; when the first net is closed, the 
Table 4.1: Event $\log$ for the internal wave acoustic survey and associated data collection events from the R/V Endeavor 307 cruise in October 1997.

\begin{tabular}{|l|l|l|l|l|}
\hline Event & Julian Time & Latitude (N) & Longitude (W) & Begin/End \\
\hline \hline CTD 08 & 287.451 & 4214.965 & 6844.765 & Begin \\
CTD 08 & 287.467 & 4214.965 & 6844.765 & End \\
\hline MOC 07 & 287.620 & 4224.04 & 6849.03 & Begin \\
MOC 07 & 287.686 & 4224.93 & 6844.22 & End \\
\hline CTD 10 & 289.535 & 4225.08 & 6844.49 & Begin \\
CTD 10 & 289.562 & 4225.08 & 6844.49 & End \\
\hline BM Pass 1 & 289.58 & 4225.638 & 6844.04 & Begin \\
BM Pass 1 & 289.67 & 4218.528 & 6840.164 & End \\
\hline BM Pass 2 & 289.68 & 4219.326 & 6840.608 & Begin \\
BM Pass 2 & 289.77 & 4229.07 & 6845.714 & End \\
\hline BM Pass 3 & 289.80 & 4231.794 & 6846.554 & Begin \\
BM Pass 3 & 289.87 & 4226.376 & 6843.152 & End \\
\hline MOC 09 & 289.896 & 4228.70 & 6845.00 & Begin \\
MOC 09 & 289.949 & 4230.97 & 6846.69 & End \\
\hline
\end{tabular}

second net is opened, and so on. This procedure allows for specific depth strata to be surveyed. Generally, net \#0 is open from the surface to the deepest point of the tow (ten to twenty meters above the bottom), the remaining nets (\#1-8) are opened and closed in succession every 25 to 50 meters during the return to the surface. The MOCNESS system also records the volume of water filtered by each net, the time that each net is opened and closed, depth, salinity, temperature, density, and fluorescence.

The nets were equipped with $333 \mu \mathrm{m}$ mesh and cod end buckets for collection of zooplankton and larval fish. Each cod end sample is then split so it will fit into quart glass jars, where the sample is preserved in a buffered formalin solution. Postprocessing of the samples consists of further splitting and silhouette photography of the animals. These photographs are then examined under a microscope and the biological organisms are measured and identified by taxonomic group (Davis and Wiebe, 1985). Abundance counts and biomass $\left(\mathrm{mg} / \mathrm{m}^{3}\right.$ ) are then calculated for each net for each taxonomic group using algorithms in Wiebe et al. (1975) and Wiebe (1988). 


\section{Zooplankton Scattering Models}

In order to interpret the volume scattering data from zooplankton, it is vital to know how an individual plankter scatters sound. For this reason, much research has been conducted on developing mathematical models that combine scattering physics and geometrical models of animal shape for various types of zooplankton (see reviews: Holliday and Pieper (1995); Foote and Stanton (2000)). These models have been compared with measured scattering from individual animals and have shown reasonably good agreement (McGehee et al, 1998; Stanton et al., 1998b). These models form the basis of both the Forward and Inverse Problems (and are presented in more detail in Appendix C).

There are generally not species specific scattering models, rather zooplankton are grouped according to similarities in their scattering physics. The three main "taxonomic types" that have been described by Stanton et al. (1998b) are: fluid-like, elastic-shelled, and gas-bearing animals. These three groups have distinctly different scattering processes and thus different mathematical models are used to describe the scattering. Fluid-like models are used for copepods, euphausiids, amphipods and other animals that have a thin shell and a body composition that has similar density and sound speed to that of sea water. Elastic-shelled models are used for animals with a hard, elastic shell such as gastropods, pteropods, or periwinkles. Pelagic pteropods are typically very small (diameters of $1 \mathrm{~mm}$ or less) when found in the water column, but scatter a large amount of sound (per unit biomass) due to their hard shell. Other strong scatterers are the gas-bearing animals such as siphonophores, where the scattering is due to the small gas bubble these animals use for flotation. At certain resonance frequencies, the scattering of the gas inclusion can be greater than that of a larger size steel ball (at other frequencies the scattering is also strong).

Within each of these three groups of scattering models, there are additional factors that need to be known in order for the models to be accurate. If these parameters (e.g., size, orientation) are not known well, then averages or estimates of them may be used, with a resulting decrease in the accuracy of the models. Not surprisingly, the 
size of the animal is directly related to the scattering strength. The relative size of the animal's body to the acoustic wavelength striking the body determines whether the scattering is in the geometric or Rayleigh scattering region. All of the mathematical models require the size of the animal as an input parameter. Thus measurements of the length of the zooplankton samples must be made or estimated.

Perhaps the most important (and least well known) input into the mathematical scattering models are the physical properties of the animal (Chu et al., 2000a,b). Most important to the physics of the problem are the density and speed of sound within the animal's body. For fluid-like animals, these parameters are generally a few percent greater than that of sea water, but it is known that changes of $3-4$ percent in the ratio of animal density and sound speed relative to sea water can change the scattering by tens of dBs (Anderson, 1950; Chu et al., 2000a,b). The models in this work use the values found by Foote (1990) for euphausiids for density and sound speed contrasts for all fluid-like animals. While this is not a perfect assumption, it is based on the best data currently available.

Finally, animal behavior in the water column can have a profound effect, on the scattered energy. Changes in animal orientation can change the cross-sectional area of the animal that scatters the sound. Thus, typically, a vertically oriented fluidlike animal will scatter less energy from a down-looking echosounder than an animal horizontally oriented in the water column (see Chapter Two). In some cases, such as elastic-shelled pelagic pteropods, the orientation of the animal can even change the number of waves that are reflected from the animal. Many animals have preferred orientations in the water column (Sameoto, 1980; Benfield et al., 2000), but the behavior of most zooplankton is still not known very well. This study will attempt to apply the limited knowledge of animal behavior in order to make the scattering models used more accurate.

Once the zooplankton scattering models are selected, they are combined with the abundance data from the MOCNESS tow to perform the Forward Problem. For each animal in a given taxonomic group, the scattering contribution for that animal is 
found using the scattering models. These contributions are summed over all animals in a taxa and then divided by the ratio of the volume sampled by the net to arrive at a scattering strength for a given volume of the ocean. The contributions from all different scatterers in the ocean are summed and the result is the predicted scattering which is then compared with the scattering measured by BIOMAPER-II.

\subsubsection{Microstructure}

Variations in temperature and salinity cause changes in the index of refraction of sound speed. These refractive index variations $(\eta)$ have been related to the acoustic scattering cross section (Seim et al., 1995). Changes in $\eta$ are related to temperature and salinity fluctuations by

$$
\eta=\frac{\Delta c}{c}=\left.\frac{1}{c} \frac{\partial c}{\partial T}\right|_{s, T} \Delta T+\left.\frac{1}{c} \frac{\partial c}{\partial s}\right|_{s, T} \Delta s
$$

where $c$ is the sound speed and salinity $(s)$ is given in concentration units. The fluctuations in $\eta$ are then related to the differential scattering cross section $(\sigma)$ by

$$
\sigma=2 \pi \kappa^{4} \Phi_{\eta}\left(k_{b r}\right)
$$

where $\kappa$ is the acoustic wavenumber $\left(\kappa=\frac{2 \pi}{\lambda}\right.$, where $\lambda$ is the acoustic wavelength), $k_{b r}$ is the Bragg wavenumber, and $\Phi_{\eta}\left(k_{b r}\right)$ is the three-dimensional wavenumber spectrum of $\eta$ evaluated at the Bragg wavenumber. Bragg scattering occurs when the separation between different scattering facets (the thickness of the microstructure layers in this case) results in the scattering from the two surfaces to be in-phase with one another. For a down-looking echo sounding system, the separation of these layers for Bragg scattering to occur is equal to half a wavelength. If the variations in temperature and

salinity occur on scales near this $\frac{\lambda}{2}$ separation distance, then constructive interference will occur resulting in a peak in the scattering spectrum. Our data are for the backscattering case, so $\sigma=\sigma_{b s}$ and $k_{b r}=2 \kappa$. Seim et al. (1995) then changes the three-dimensional wavenumber spectra to a one-dimensional, one-sided spectrum $(\phi)$ 
such that

$$
\Phi=-\frac{1}{4 \pi k} \frac{d}{d k} \phi(k)
$$

which can be used to relate the backscattering cross section to the one-sided spectrum

$$
\sigma_{b s}=-\frac{k_{b r}^{3}}{32} \frac{d}{d k} \phi_{\eta}\left(k_{b r}\right)
$$

and $\phi_{\eta}$ is then estimated to be dependent upon the spectra of temperature and salinity

$$
\phi_{\eta}=a^{2} \phi_{T}+b^{2} \phi_{s}+2 a b \phi_{s T}
$$

where $\phi_{T}$ and $\phi_{s}$ are the one-dimensional spectra of temperature and salinity, $\phi_{s T}$ is a co-spectrum, and $a$ and $b$ are the relative importance of temperature and salinity to variations in sound speed $\left(a=\left.\frac{1}{c} \frac{\partial c}{\partial T}\right|_{s, T} b=\left.\frac{1}{c} \frac{\partial c}{\partial s}\right|_{s, T}\right)$.

Temperature and salinity microstructure occur throughout the oceans and the processes that cause and result from the microstructure are of great importance to oceanographers (Gregg, 1987; Thorpe, 1987). In many cases, turbulence in the water column will result in these temperature and salinity variations. High-frequency acoustic measurements were suggested as a means to study turbulence over forty years ago (Batchelor, 1959), however only in this last decade have scientists actually provided theoretical models for this acoustic scattering process. These scattering models have inputs which are related to turbulence, such as $\epsilon$ and $\chi$ (the dissipation rates of turbulent kinetic energy and temperature variance respectively), however there are other processes besides turbulence that can cause microstructure (Gregg, 1987). Salt fingers or diffusion can provide the variations in microstructure without the presence of turbulence. While the volume scattering strength has been estimated from microstructure measurements made by profiling instruments (Seim et al., 1995; Seim, 1999), until this work there has not been a study relating the acoustic scattering to both the biology and microstructure present.

It is difficult to measure the amount of microstructure from a towed instrument, such as BIOMAPER-II. Ideally, sensors projecting far from the tow-body would mea- 
sure the temperature, salinity, and three dimensional fluid velocities which would allow for direct calculations of microstructure and turbulent parameters the aforementioned $\epsilon$ and $\chi$ ). However, the BIOMAPER-II and MOCNESS systems currently do not have this type of instrument on board. Instead, indirect methods of estimating these inputs to the scattering model will be made from CTD data collected by the ESS system on BIOMAPER-II and the MOCNESS.

\section{Physics-based Scattering Model for Microstructure}

In the last decade several physics-based scattering models have been developed to describe the scattering from temperature microstructure (Goodman, 1990; Seim et al., 1995). Recently, Seim (1999) published an improved model that includes scattering contributions from both temperature and salinity microstructure. A copy of the MATLAB code of this model has been generously provided by Dr. Seim and is used in this analysis.

The scattering model used requires four input parameters: $t(z)$, the temperature profile; $s(z)$, the salinity profile; $\epsilon$, the dissipation rate of turbulent kinetic energy; and $\chi$, the dissipation rate of temperature variance. The temperature and salinity profiles are measured directly by the ESS or CTD data, and a method to estimate $\epsilon$ and $\chi$ will be described.

Temperature and salinity profiles are collected by the ESS system (installed on both BIOMAPER-II and the MOCNESS) which consists of an underwater pump, SeaBird temperature and conductivity probes, and other sensors. With a sampling rate of $0.25 \mathrm{~Hz}$, the instruments send data to a ship-board computer where they are plotted and recorded to the hard drive via a Visual Basic program. The limiting factors in using the CTD data from the MOCNESS are the low sampling rate and the fact that the net system is moving not only vertically but also horizontally. A MOCNESS tow takes approximately one hour to go from the deepest point (around 200 meters in the Gulf of Maine) to the surface. During this time, the ship is moving at approximately 1 knot, which means that the net tow covers a horizontal range 
of approximately 1.8 kilometers. For comparison a CTD cast covers a horizontal range on the order of 10 - 100 meters, so horizontal movement is much less for this instrument. The importance of vertical resolution and horizontal movement is because the temperature and salinity data are used to characterize a specific region of the water column. So the resolution should be high and lateral movement low to accurately measure a particular area.

The temperature and salinity profiles are used to estimate the other inputs into the acoustic scattering model for microstructure. A full description of this method is provided by Dillon (1982), which details how to use instabilities in the density profile of the water column to provide a scaling, the Thorpe length $\left(L_{T}\right)$, which has been found to be proportional to the Ozmidov length which in turn is proportional to the dissipation rate of turbulent kinetic energy. A Thorpe length can be found by re-ordering the density profile such that it is stable (higher density water is deeper than lower density water), the Thorpe length is simply the root mean square vertical displacement that a water parcel would undergo in moving from the measured profile to a theoretical "stable" profile. This was done by sorting the density profile and finding the rms displacement that had occurred. The Thorpe length calculations are averaged over multiple depth bins (selected by hand in this work) in the water column, in order to reduce the variability.

Once the Thorpe length has been found, it can be related to the Ozmidov scale, $L_{o}$. Dillon (1982) showed that for three different oceanic conditions that the Thorpe length was directly proportional to the Ozmidov length by the relationship

$$
L_{o}(z)=0.8 L_{T}(z)
$$

and the Ozmidov length is a function of the dissipation rate of turbulent kinetic energy and the buoyancy frequency $(N)$

$$
L_{0}(z)=\left(\frac{\epsilon(z)}{N^{3}(z)}\right)^{\frac{1}{2}}
$$


The buoyancy frequency is found by using the gradient of the density profile

$$
N^{2}(z)=-\frac{g}{\rho(z)} \frac{\partial \rho(z)}{\partial z}
$$

where $\rho$ is the fluid density, $g$ is the acceleration due to gravity, and $z$ is the vertical coordinate and is positive upward. Once the Thorpe length is estimated, then $\epsilon$ can be found by combining the above equations. It should be noted that these estimates for $\epsilon$ should be considered rough values (due to the low sampling rate of the ESS system), and are used only because there were no instruments aboard to make direct measurements of these imputs.

Finally, $\chi$ is estimated by

$$
\chi(z)=\gamma \epsilon(z) \frac{2 \bar{T}_{z}}{N^{2}(z)}
$$

where $\gamma$ is the mixing efficiency ( $\gamma$ is assumed to equal 0.2 following Seim (1999) and Gregg (1987)) and $\bar{T}_{z}$ is the mean vertical gradient of the temperature profile. Direct measurements of $\chi$ require temperature probes with very fast response times and are difficult to obtain.

The scattering model used divides the wavenumber (or frequency) spectrum into two regions: the inertial-convective and viscous-convective. The parameter $A$ is chosen so that these two regions are equal at $k=k_{\text {int }}$. The model is

$$
\begin{aligned}
\sigma= & A \frac{5}{96} \frac{\chi}{\epsilon^{\frac{1}{3}}} k_{b r}^{\frac{1}{3}}\left(a^{2}+\frac{b^{2}}{\delta^{2}}+\frac{2 a b}{\delta}\right) \quad \text { for } \quad k \leq k_{i n t} \\
\sigma= & q\left(\frac{\nu}{\epsilon}\right)^{\frac{1}{2}} \frac{\chi k_{b r}}{32}\left[a^{2} e^{-\zeta^{2} / 2}+\frac{b^{2}}{\delta^{2}} e^{-\zeta^{2} / 2}+\frac{a b \Gamma}{\delta\left[g(\zeta) g\left(\zeta_{s}\right)\right]^{\frac{1}{2}}}\right] \text { for } \quad k>k_{i n t} \\
& \text { where } \Gamma=\left(\left(\frac{D_{s}}{D}\right)^{\frac{1}{4}} g(\zeta) e^{-\zeta^{2} / 2}+\left(\frac{D}{D_{s}}\right)^{\frac{1}{4}} g\left(\zeta_{s}\right) e^{-\zeta^{2} / 2}\right) \\
& a=\left.\frac{1}{c} \frac{\partial c}{\partial T}\right|_{S, T}, b=\left.\frac{1}{c} \frac{\partial c}{\partial s}\right|_{S, T}, \delta=\frac{\frac{\partial T}{\partial z}}{\frac{\partial s}{\partial z}}, k_{*}=\frac{\left(\frac{\epsilon}{\nu^{3}}\right)^{\frac{1}{4}}}{8}, k_{i n t}=\frac{5^{\frac{3}{2}}}{3} k_{*} \\
& \zeta=(2 q)^{\frac{1}{2}} \frac{k}{k_{d}}=\text { dissipation rate of scalar variance, }
\end{aligned}
$$


$g(\zeta)=\zeta\left[e^{-\frac{\zeta^{2}}{2}}-\zeta \int_{\zeta}^{\infty} e^{-\frac{x^{2}}{2}} d x\right], \nu=$ viscosity, $c=$ conductivity

$D=$ scalar diffusivity for temperature $D_{s}=$ scalar diffusivity of salinity

$k_{\text {int }}$ is where inertial-convective and viscous-convective subranges intersect, and $k_{b r}=$ Bragg wavenumber

\section{Application of the Scattering Model}

As mentioned previously, the cruise was not equipped to make direct measurements of $\chi$ and $\epsilon$. Therefore in order to use the acoustic scattering model, we estimate these two parameters based upon the temperature, salinity, and density profiles obtained by the MOCNESS tows and CTD casts. Problems associated with the MOCNESS system are a low sampling rate and the fact that the instrument is moving horizontally as well as vertically during the profile. The low sampling rate $(0.25 \mathrm{~Hz})$ limits the vertical resolution of the profiles which could cause problems in the estimates of Thorpe lengths since very small scale variations in density would not be detected. Additionally, the horizontal movement of the instrument means that our profile is really a transect through (possibly) different hydrographic regions. The advantage of the MOCNESS measurements is that they are collected at the exact same points that the net is collecting samples and the acoustic system is measuring.

The disadvantage of the CTD casts is the converse, they occur in regions nearby, but not at the point of the acoustic and net measurements. Water column hydrography (especially in regions with internal waves) can change quite rapidly in both space and time, so even though the high sampling rate $(24 \mathrm{~Hz})$ of the CTD cast offers finer vertical resolution (roughly $1 \mathrm{~cm}$ ), there is much uncertainty in whether the measurements are applicable to the region of the MOCNESS tow.

\section{Inversion for $\epsilon$}

Due to the possibility of errors in our method of estimating values of $\epsilon$ from the ESS or CTD data, the multiple frequency acoustic data was inverted using the theoretical scattering model to estimate values of $\epsilon$. This test was done to check the "reason- 
ability" of our estimates of $\epsilon$ and $\chi$ : to determine what levels of turbulence would be needed to cause the acoustic scattering that was measured. Once the net tow biological abundance data has been enumerated, the Forward Problem can be calculated. Theoretically, if microstructure is causing acoustic scattering, the measured values of scattering will be greater than the predictions from the Forward Problem calculations (which combine zooplankton scattering models and zooplankton abundance). If we assume that there are no other scattering processes occurring in the ocean other than biology and microstructure, then the difference between the measured and theoretical (from zooplankton) scattering must be caused by scattering from microstructure.

A wide range of values of the dissipation rate of turbulent kinetic energy $(\epsilon=$

$10^{-9}-10^{-3}$ ) were used as inputs into many runs of the acoustic scattering from microstructure model (as before $\chi$ was found using Equation 4.11). The model output is volume scattering strength versus depth for the acoustic frequencies that BIOMAPER-II uses. The model output scattering that matches the "leftover" scattering from the Forward Problem calculation allows us to invert the acoustic data for estimates of turbulence in the water column, specifically values of $\epsilon$ at the depths that the net tow sampled.

\subsection{Results}

Three transects were made through the internal wave region on 16 October 1997 (Figure 4-5), the first pass was from 1400 - 1600 (local time), the second pass was from 1620 - 1830, and the final pass was from 1910 - 2050. The BIOMAPER-II instrument was at varying depths for the three passes $(5,25$, and 50 meters respectively) in order to use the VPR instrument to examine the different layers. Data from the VPR system are presented in Appendix B, however the sampling volume of the system was set too small (corrected for in later cruises) for any quantitative comparison with the acoustic or net tow data. While the internal wave was clearly seen in the acoustic data during the initial transect, in each successive pass the structure of the wave diminished (Figure 4-6). Immediately upon completion of the third pass, MOCNESS tow \#9 was 
conducted which lasted from 2130 - 2245. Presumably some vertical migration had occurred between the acoustic transects through the wave and the MOCNESS tow. CTD cast \#10 was conducted immediately before the acoustic survey and is presented to show changes in the hydrographic structure of the water column. For comparison, a similar analysis was conducted on the acoustic, environmental, and net tow data for this region, collected two days earlier (14 October 1997) during MOCNESS tow \#7 and CTD cast \#8 (see Appendix A).

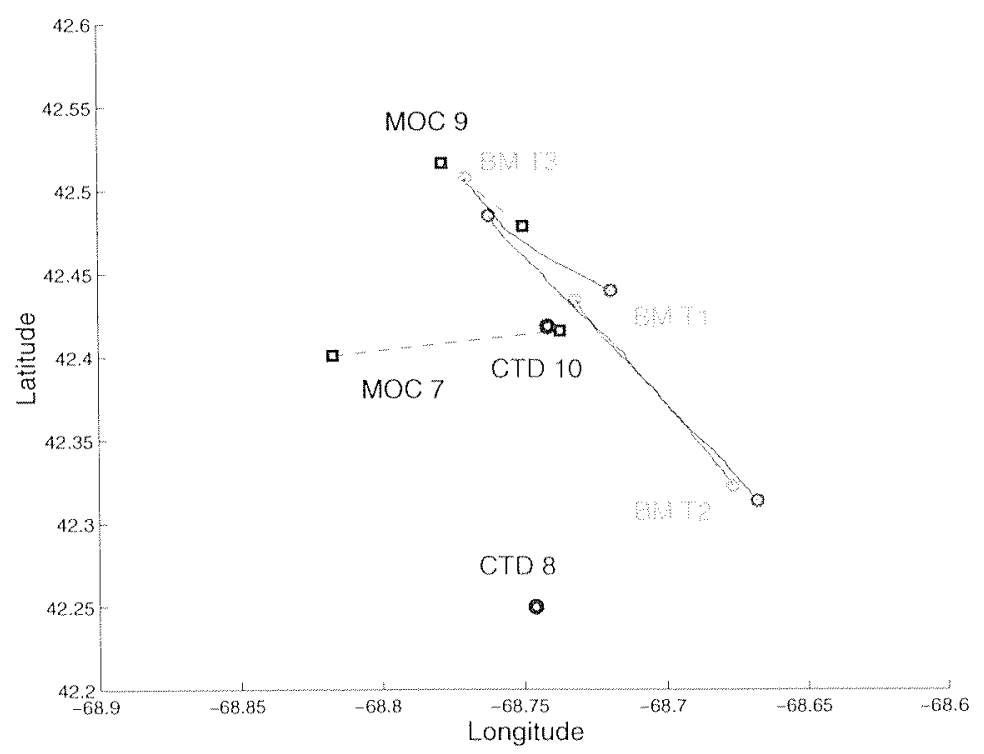

Figure 4-5: Map of the various MOCNESS, CTD, and acoustic transects that occurred in the area of the internal wave study. Circles indicate acoustic transects through the internal wave and CTD stations, while squares indicate MOCNESS tows. CTD $\$ 8$ and MOCNESS $\$ 7$ occurred on yearday 287 (14 October 1997), while all other measurements were made on yearday 289. The depth of this area (the eastern edge of Wilkinson Basin) is approximately 200 meters.

\subsubsection{Acoustic, Environmental and Net Tow Data for 16 October 1997}

\section{Acoustic Scattering Data}

As the MOCNESS tow was being conducted, the BIOMAPER-II instrument was collecting acoustic data while being towed at a depth of approximately 5 meters beside the ship. The acoustic data are offset horizontally from the MOCNESS collection by the amount, of wire out on the net tow, however it is believed that both systems 


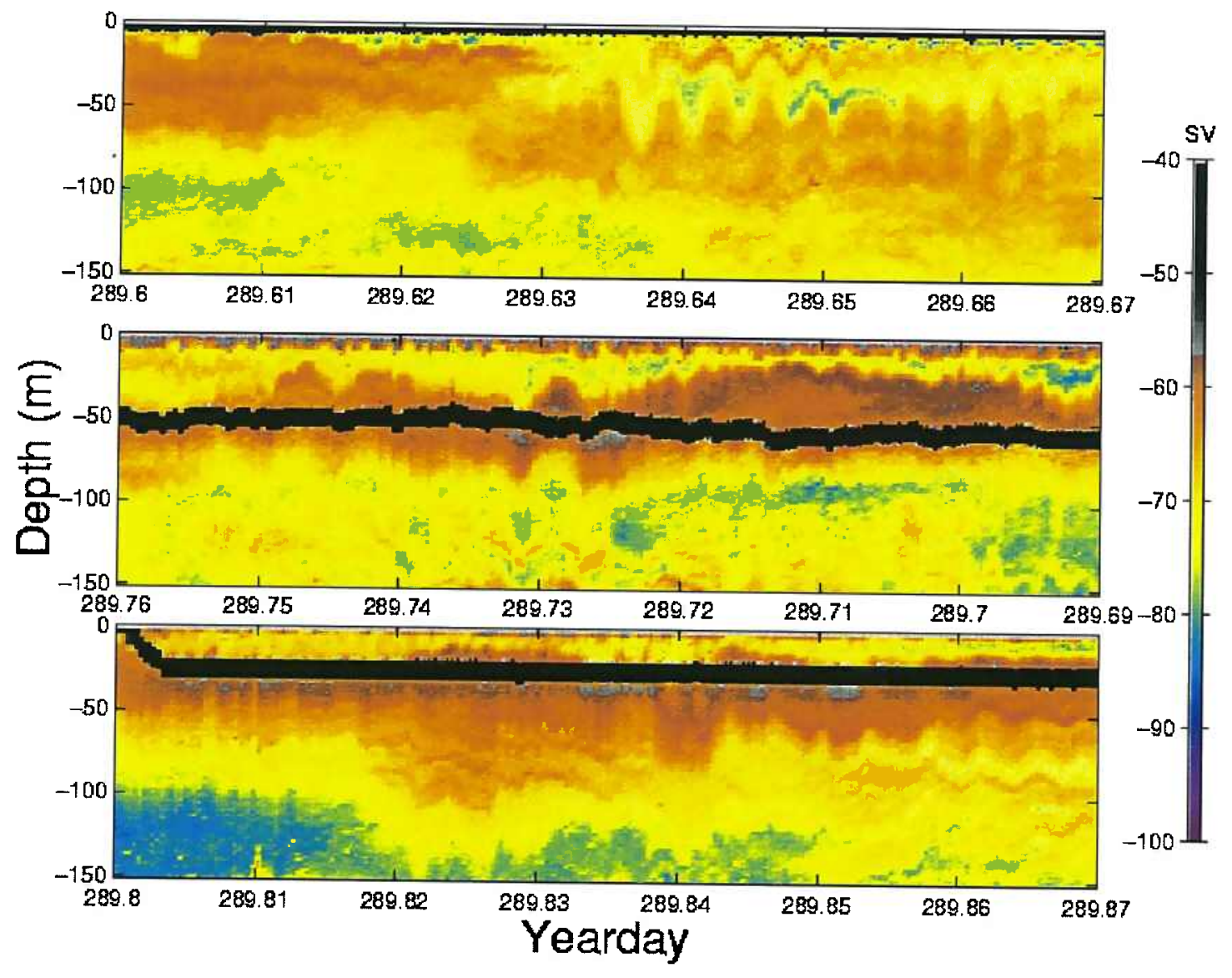

Figure 4-6: The three acoustic transects $(120 \mathrm{kHz}$ data shown) through the internal wave field. The first (top), second (middle) and third (bottom) surveys were made consecutively with a $180^{\circ}$ turn in the ship between each transect. While a strong internal wave was seen in the first transect, the second and third passes do not show the wave nearly as well. BIOMAPER-II was towed at varying depths $(5,50$, and 25 meters respectively) through the internal wave in order to use the VPR data to characterize the different layers, unfortunately the distinct layers were not present during the second and third passes. 
are sampling a similar region of zooplankton. The information from four acoustic transducers show a strong near-surface scattering layer that is strongest at lower frequencies (Figure 4-7).

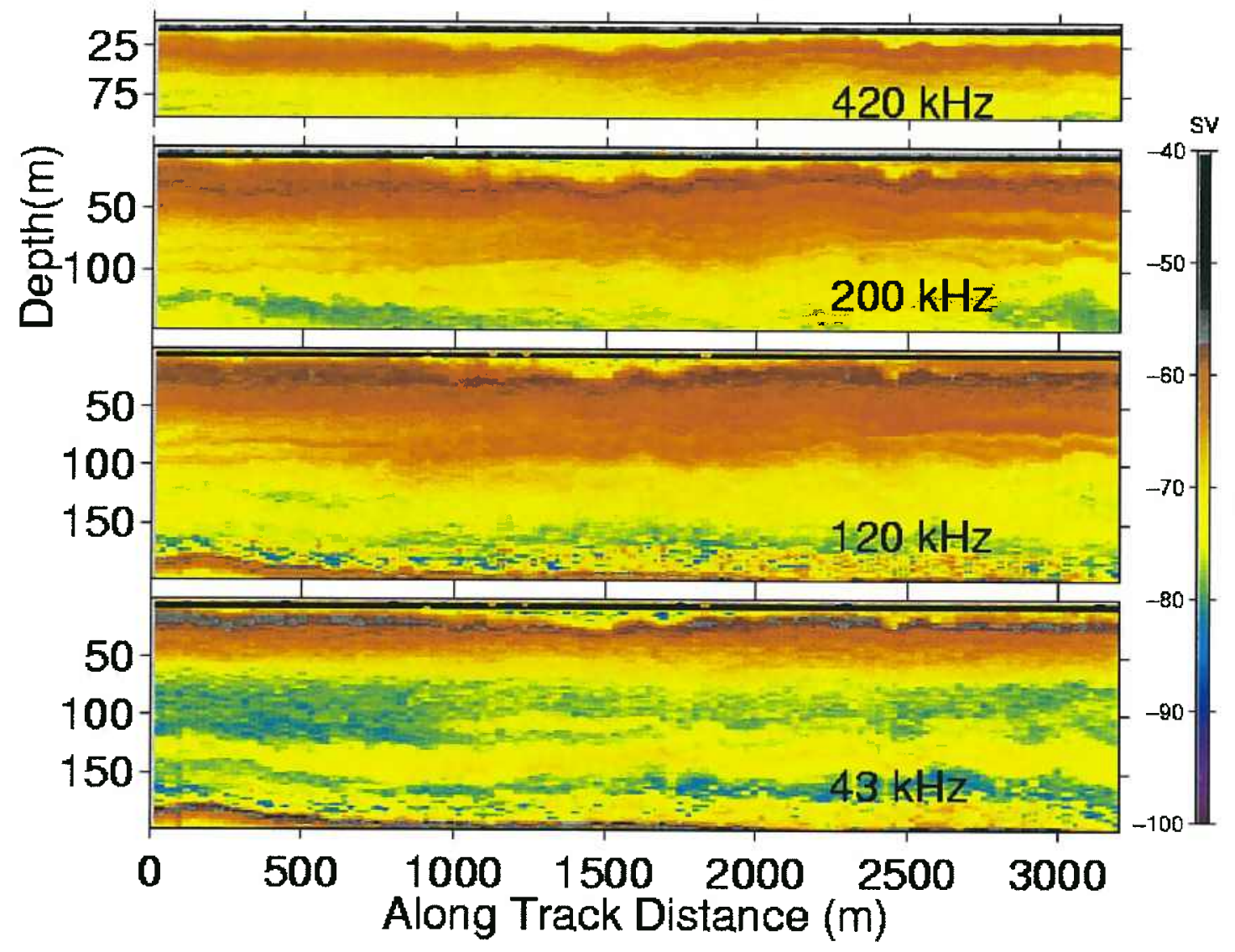

Figure 4-7: Echogram of the volume scattering strength for the four frequencies $(43,120,200$, and $420 \mathrm{kHz}$ from top to bottom) of BIOMAPER-II collected simultaneously during MOCNESS \#9. There is a strong scattering layer near the surface which shows up on all four frequencies (but is strongest at the lower frequencies), and a weaker (but still quite strong) layer of scattering at approximately 125 meters which is detected by the lower frequencies.

In order to compare the acoustic regions with the MOCNESS information, the transect of the net can be overlaid on the acoustic plot to show which nets sampled which regions. The $120 \mathrm{kHz}$ curtain plot shows increasingly strong scattering from nets \#4 - 6, with a huge scattering layer that is sampled mostly by net \# 7 (Figure 48). There are also regions of strong scattering (although very patchy) occurring very close to the bottom. These near-bottom scatterers are presumed to be fish which 


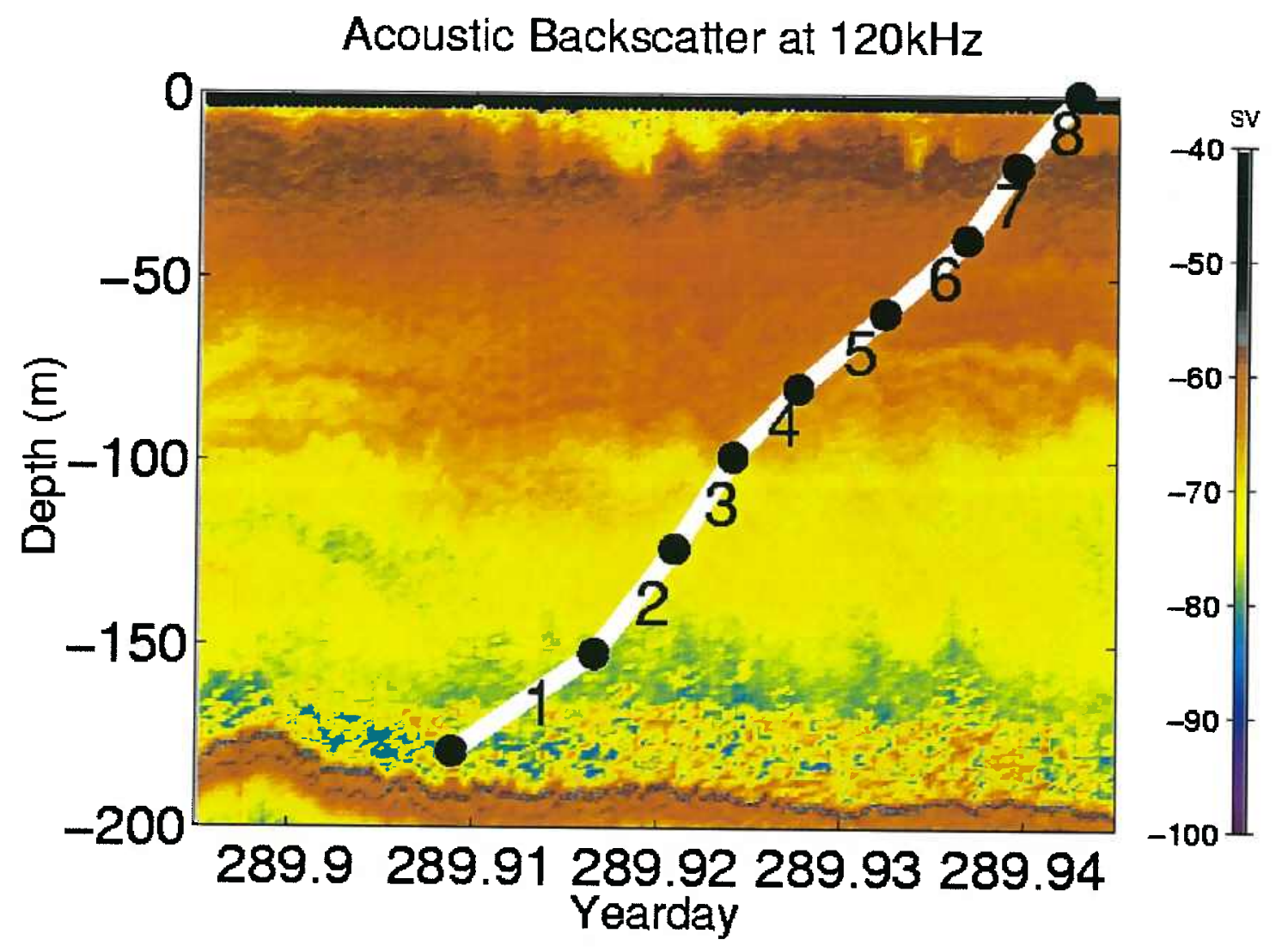

Figure 4-8: Volume scattering strength at $120 \mathrm{kHz}$ for MOCNESS \#9. The strong surface scattering layer is sampled by net \#7, while there is also strong scattering for MOCNESS nets \# 4 - 6 .

If the slope information from the multiple frequency scattering data is used, a different picture of the water column is formed. The first-order slope of the scattering spectrum (using the 43,120 , and $200 \mathrm{kHz}$ transducers only) shows that a region of positive slope (consistent with scattering from fluid-like scatterers) occurs during nets \# 4 - 5 (with some overlap in nets \# 3 and 6) (Figure 4-9). Negative slope regions occur at the depths sampled by nets \# 2 and 7 . Net \# 8 has a positive slope but the data are somewhat difficult to interpret because the instrument was towed at a depth of 5 meters and may introduce artifacts (such as surface scattering and acoustic near-field effects).

If four frequencies $(43,120,200$, and $420 \mathrm{kHz})$ are used to find the slope parameter, 


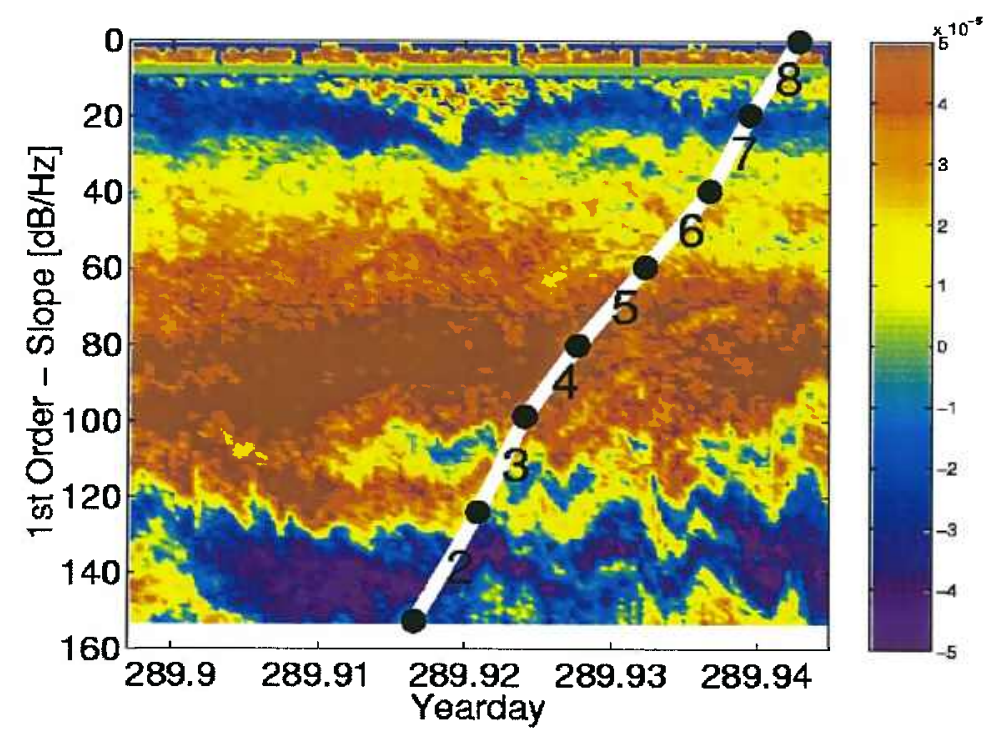

Figure 4-9: Three frequency slope calculation from the BIOMAPER-II acoustic data collected during MOCNESS \#9. The water column is divided into distinct regions which have either positive or negative slopes. Nets \#4-5 sample regions with positive slope, while nets \#2 and 7 appear to sample regions with negative slopes.

a similar picture is found (Figure 4-10). While limited to the upper 100 meters of the water column, due to the range limits of the $420 \mathrm{kHz}$ transducer, these data show positive slope regions occurring during nets \# 4 and 5 , with negative slope regions for nets \# 6 and 7, while again net \# 8 has both positive and negative slopes.

Second-order slope calculations (curvature and concavity) also indicate distinct regions in the water column. The three frequency calculation of curvature indicates that nets \# 4 and 5 (and partially 3 and 6) are in a different region than nets \# 2 and 7 (Figure 4-11). However the concavity calculations shows little variation in the water column. If four frequencies are used to find the curvature and concavity of the scattering spectrum, then these data clearly show that nets \#4 and 5 occur in a different region than net \# 7, with nets \# 6 and 8 overlapping both positive and negative curvature and concavity values (Figure 4-12).

There are additional complications in that horizontal variations in the slope calculations occur over very short time scales, and the MOCNESS tow overlays may not be accurately placed over the slope data due to uncertainty in the MOCNESS position 


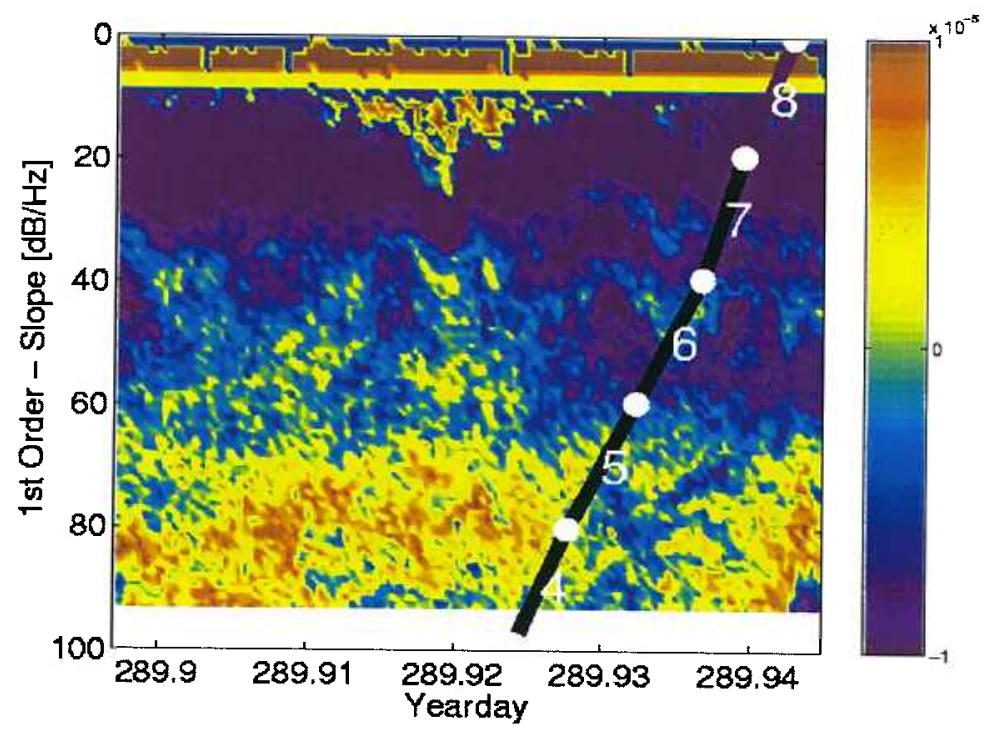

Figure 4-10: Four frequency slope calculation from the BIOMAPER-II acoustic data collected during MOCNESS \#9. The upper water column dominated by a negative slope region, with nets \#4 and 5 sampling regions with possibly positive slope.
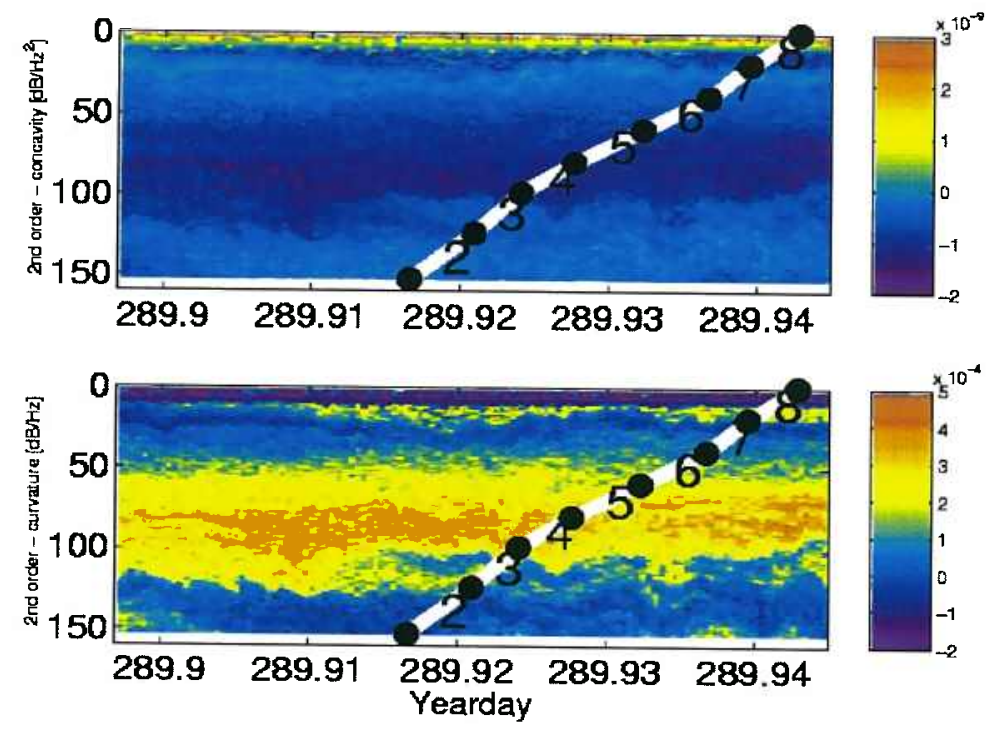

Figure 4-11: Three frequency second order slope (curvature and concavity) of the scattering strength versus frequency measurements from the BIOMAPER-II acoustic data collected during MOCNESS \#9. Both slope parameters divide the water column into similar regions, although there is less differentiation from the concavity parameter. These regions are the same as those identified by the first order slope parameter. 

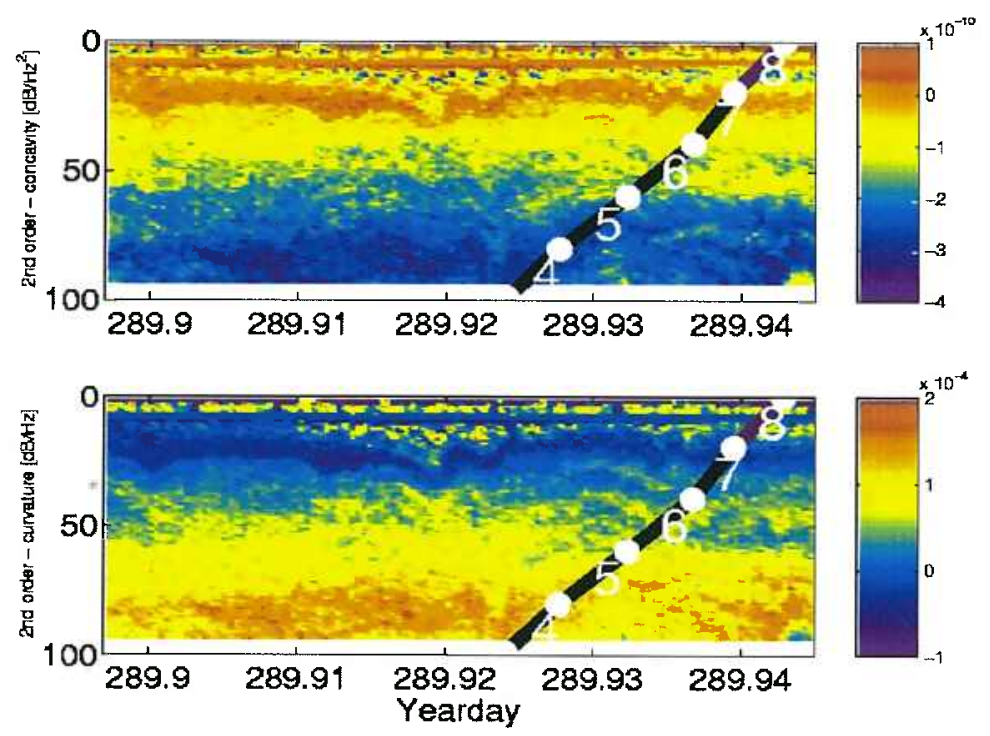

Figure 4-12: Four frequency second order slope (curvature and concavity) of the scattering strength versus frequency measurements from the BIOMAPER-II acoustic data collected during MOCNESS \#9. Both slope parameters divide the water column into similar regions. These regions are the same as those identified by the first order slope parameter.

relative to BIOMAPER-II. It is obvious that the number of frequencies used in this analysis plays a large role in determining the regions that are distinguished by the different slope. While the four frequency plots offer additional spectral information, the limited depth range is a hindrance in comparing these data to the MOCNESS tows which generally go to 200 meters depth.

\section{MOCNESS \#9}

The MOCNESS was lowered to 180 meters depth and was brought to the surface with nets closed and opened at 152, 124, 99, 80,60,40, 20, and 2 meters. The samples were dominated by an enormous number of salps $\left(2,500\right.$ animals $\left./ \mathrm{m}^{3}\right)$ at the near-surface (net \# 7 covered 40 - 20 meters depth) which led to a large amount of biomass, nearly $1 \mathrm{~g} / \mathrm{m}^{3}$ (Figure 4-13). There was also a substantial amount of biomass from approximately 125 - 80 meters which was dominated by euphausiids (Figure 4-14). The salp surface layer was an unusual occurrence on this cruise and no other tow had such a large amount of biomass. The surface net (\#8) also contained 
fragments of siphonophores.

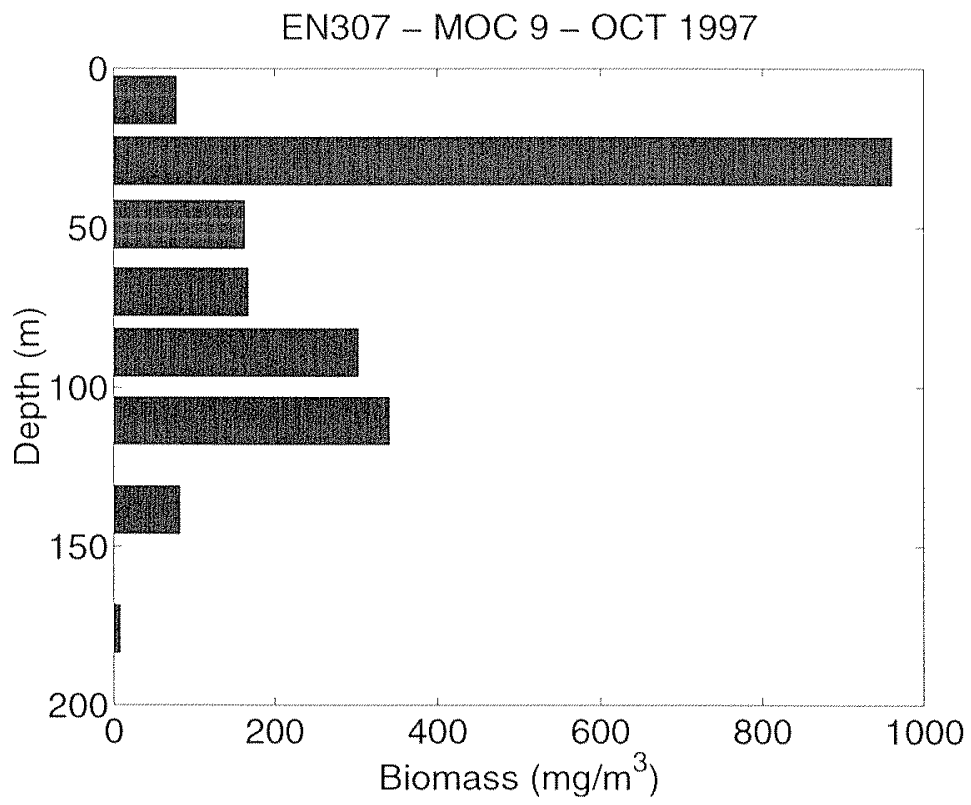

Figure 4-13: Biomass estimates from MOCNESS \#9. Net \#7 has an enormous amount of biomass dominated by salps. The deeper peak in biomass (from 90 - 140 meters) is typical of most the deep basin tows in the Gulf of Maine.

The abundance data indicate that copepods were by far the most frequently found zooplankton, which is not surprising given their small size and almost ubiquitous presence in the waters of the Gulf of Maine (Figures 4-15 and 4-16). Larger copepods (lengths greater than $2.5 \mathrm{~mm}$ ) were found in the deeper waters (180 - 80 meters), while salps on a numerical basis (\# of animals $/ \mathrm{m}^{3}$ ) made up $25 \%$ of the surface net sample.

\section{Water Column Hydrography}

The hydrography of the water column is shown from data collected by the ESS system on-board MOCNESS \# 9. The data show a well-mixed region in the top 20 meters of the water column with a large gradient in temperature, salinity and density occurring in the next 10 meters (Figure 4-17). There are several regions of mixing (evidenced by horizontal excursions in the temperature and salinity profiles) occurring between $60-100$ and $140-180$ meters; although there are smaller instabilities that occur 


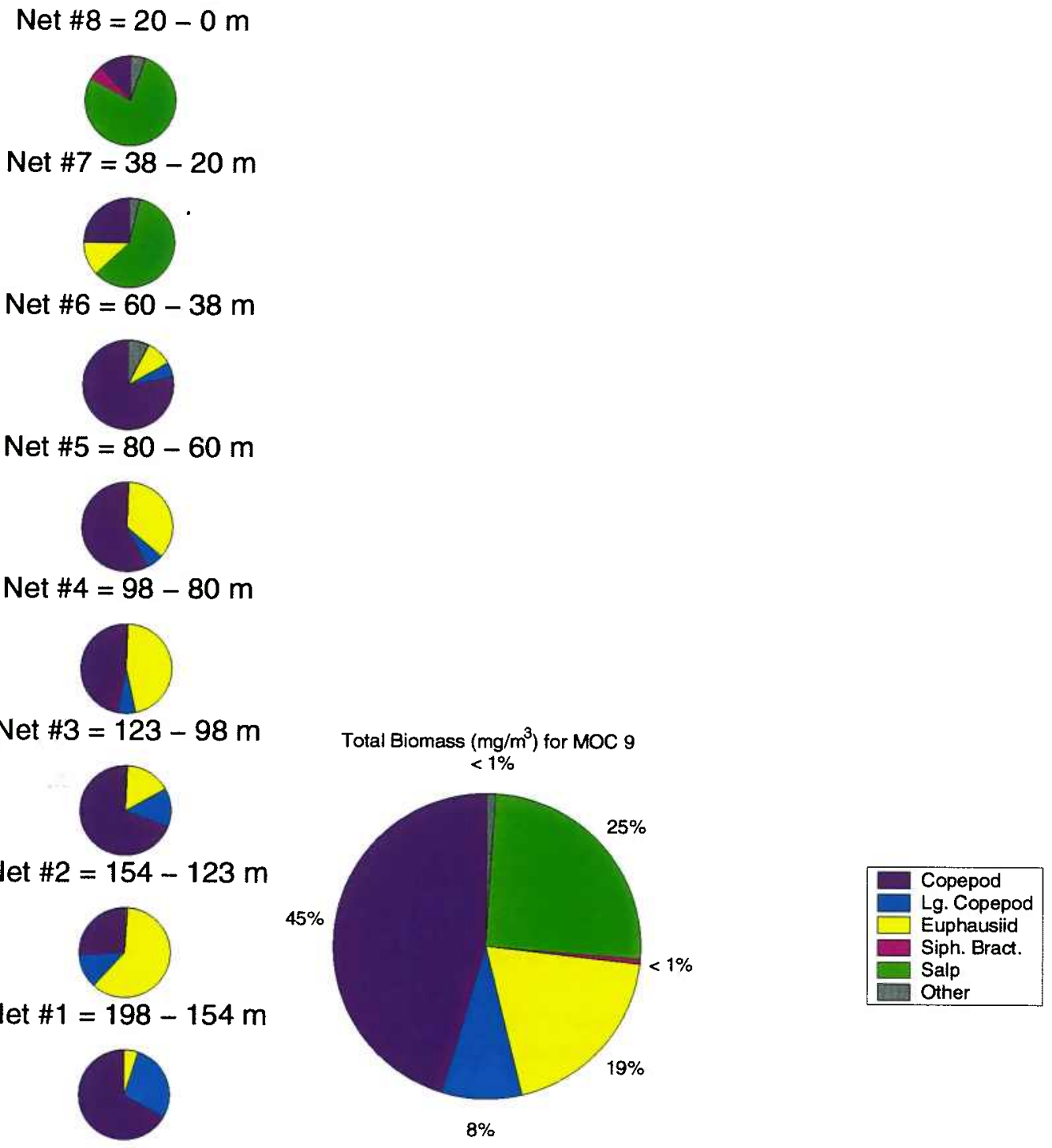

Figure 4-14: Composition of biomass for each net and overall by taxonomic or scattering type. The upper two nets were dominated by salps while euphausiids contributed the most to biomass in the lower nets. Copepods were found throughout the water column and contributed to the overall biomass a substantial amount. 


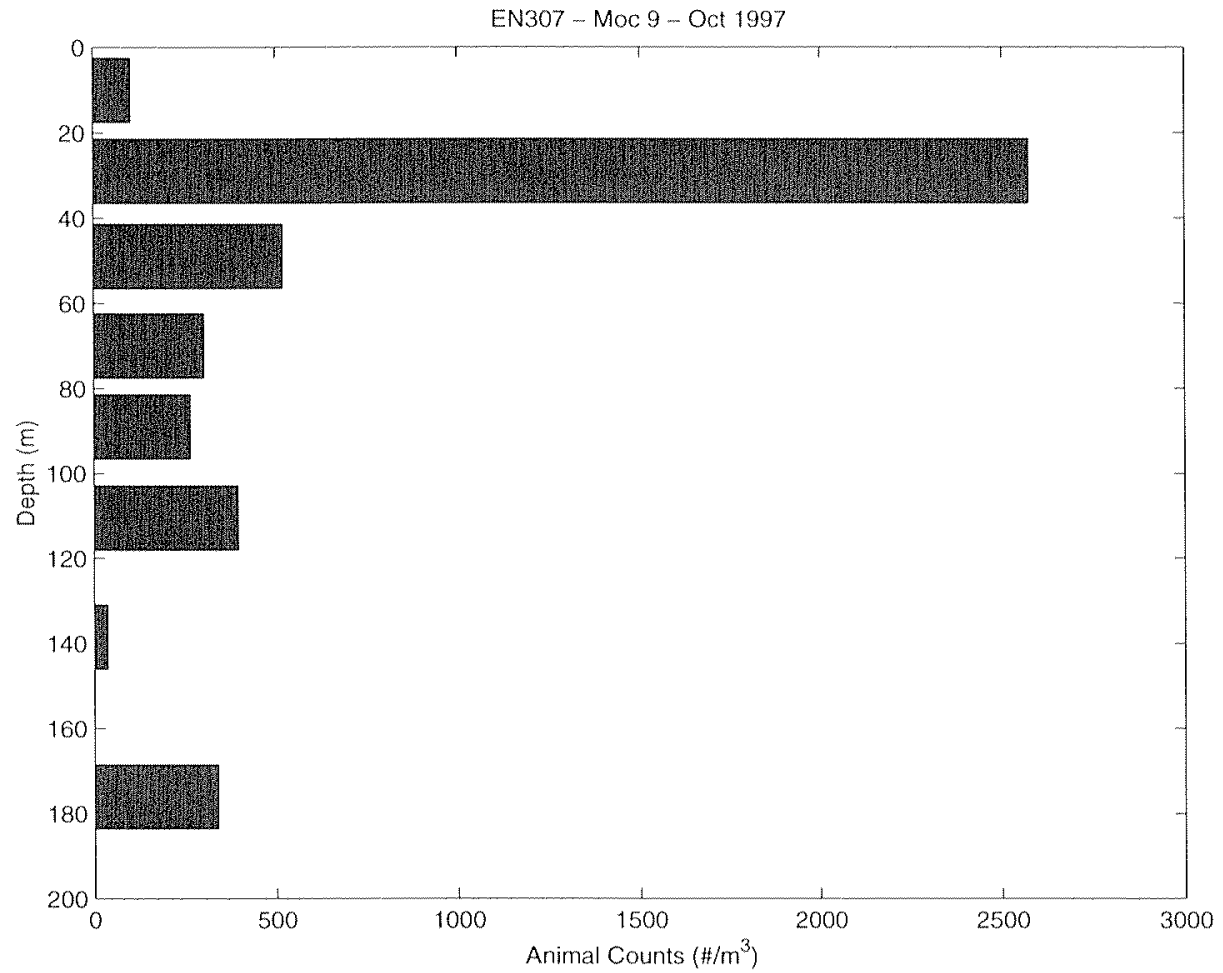

Figure 4-15: Numerical abundance (animals $/ \mathrm{m}^{3}$ ) for each net from MOCNESS \#9. While the subsurface peak is similar to that for the biomass data, the lower water column is quite different (as would be expected) than the biomass data. 


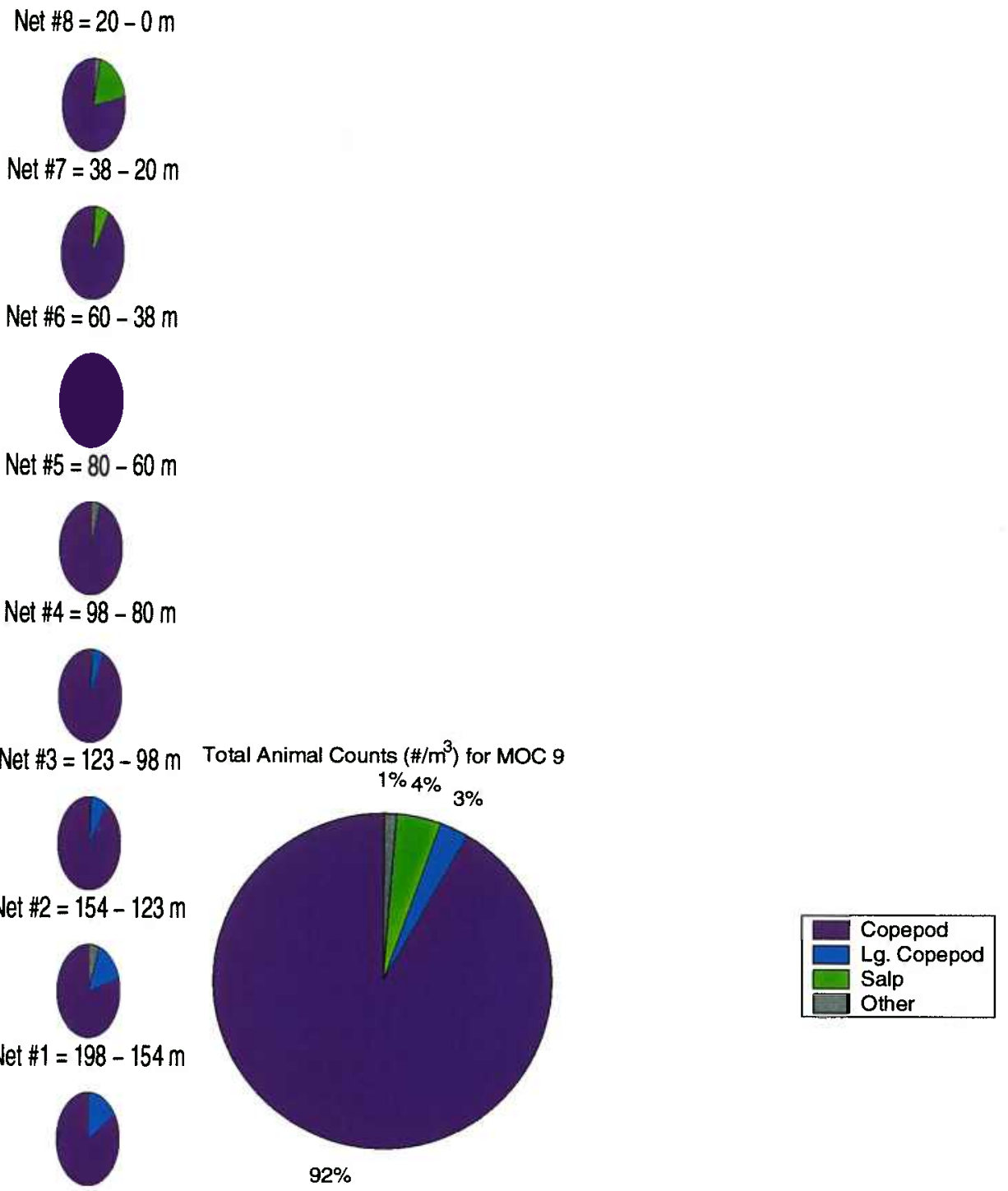

Figure 4-16: Taxonomic composition of the numerical abundance data for MOCNESS \#9 for each net and overall. Copepods were by far the most abundant animal sampled, however their contribution to the overall biomass is usually much smaller (due to the copepods small size) than that for the other taxa types that are not as numerically abundant. 
throughout the profile. The data collected by the ESS system are sampled at 0.25 $\mathrm{Hz}$ which results in a data point approximately every $20-50 \mathrm{~cm}$ during a typical BIOMAPER-II tow-yo. Therefore many instabilities or mixing events that are smaller than 0.5 meter will not be resolved by these data.

Data collected from CTD cast \# 10 which occurred before the acoustic survey of the internal waves began were also used in this analysis (Figure 4-5). These higher resolution (24 $\mathrm{Hz}$ sampling rate) data resolve water column variations on the centimeter scale (Figure 4-18). These data show large variations in the upper 20 meters with smaller overtumings (higher density water located above lower density water) occurring throughout the rest of the water column. However, this cast is from a different region than where the MOCNESS tow occurred and was taken before evidence of internal waves was seen, so its applicability (despite its high resolution) to our MOCNESS data is not known. It is possible though that the data from CTD \#10 represent a "before" and the data from MOCNESS \#9 provide an "after" picture of the effects of an intemal wave propagating through the water column.

\section{Forward Problem Calculations}

Before the Inverse Problem can be solved, the Forward Problem must be well understood and modeled. The Forward Problem consists of taking the abundance and distribution of different zooplankton taxa and applying acoustical scattering models for each sampled individual in each taxa. These results are then divided by the volume of water sampled by the net system which results in a value for $s_{v}$. This "predicted" level of volume scattering is then plotted against the value of volume scattering measured by BIOMAPER-II. The measured values are averaged over the depth and time ranges that each of the nets were sampling.

If the measured values of volume scattering are smaller than the predicted values, there is an "over-estimate" by the Forward Problem calculation (Figure 4-19). The likely reason this would occur is that the acoustical scattering models predict scattering that is stronger than the scattering from an individual animal. Either changes 

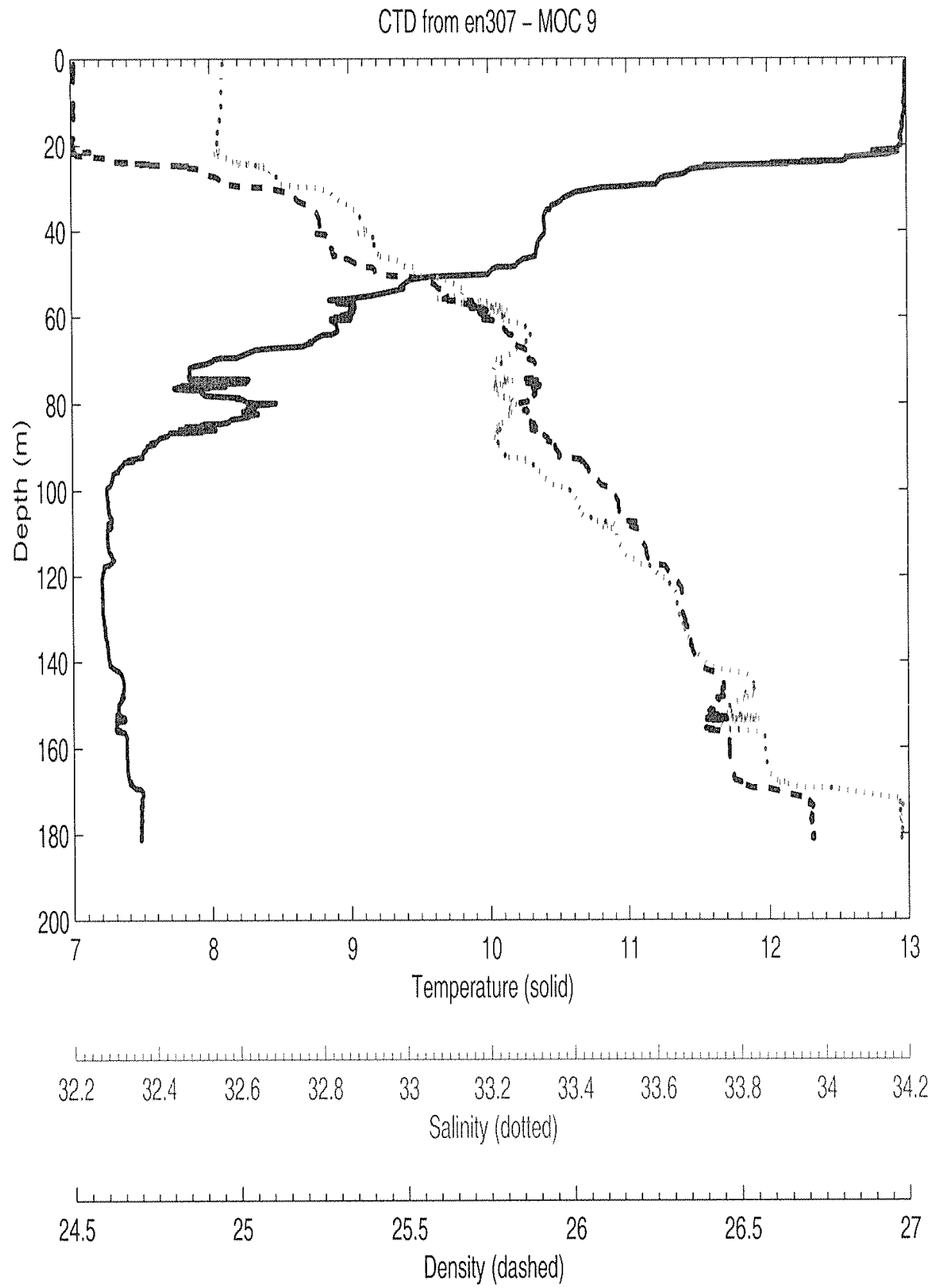

Figure 4-17: Temperature, salinity, and density profiles for the water column sampled by the ESS system on board MOCNESS tow \#9. The upper 20 meters appear to be well mixed, as does a region between 140 and 180 meters. There appear to be many "overturnings" in the density profile which suggest that mixing events are occurring. 

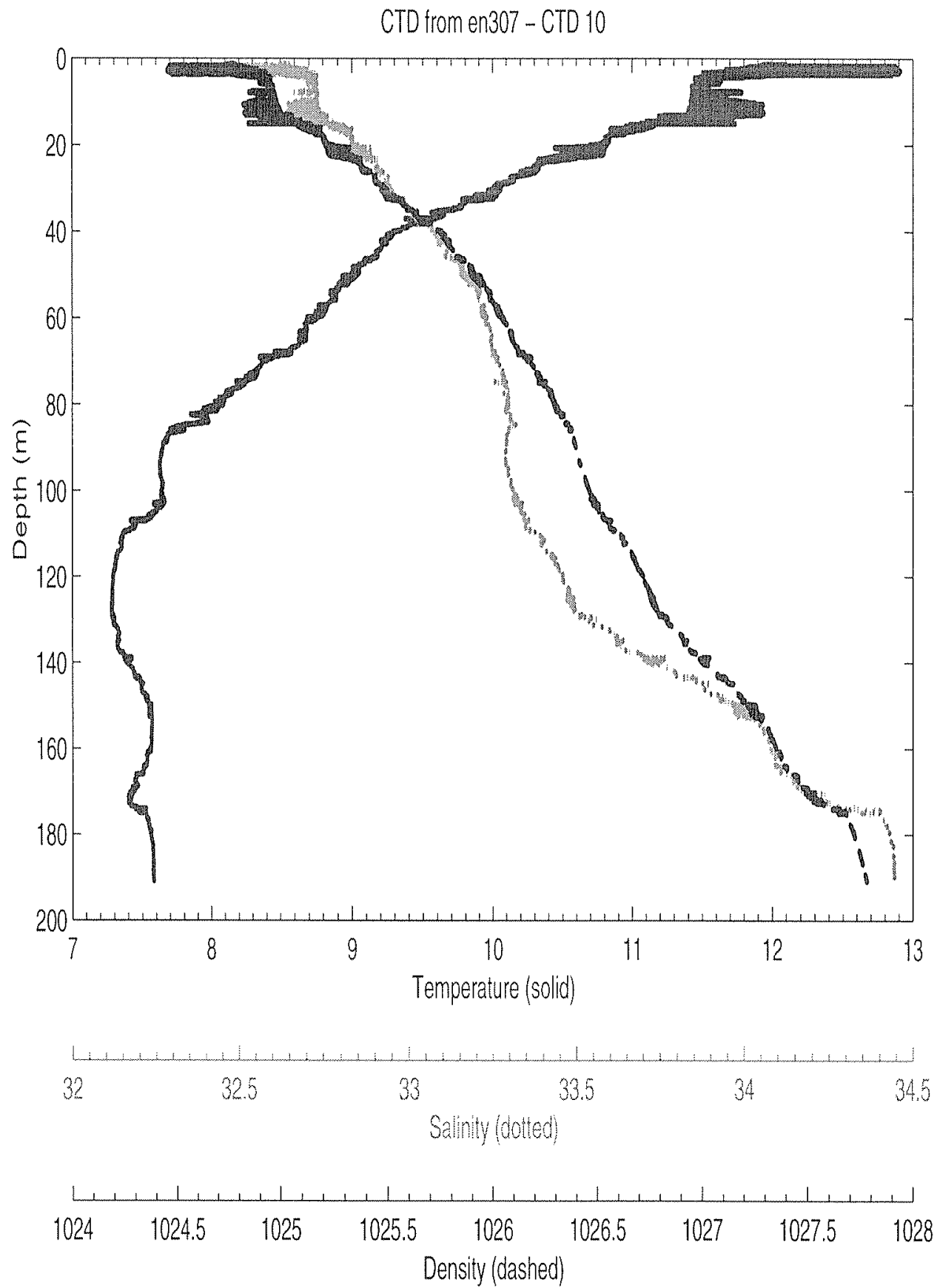

Figure 4-18: Temperature, salinity, and density profiles for the water column sampled by CTD cast \#10 conducted immediately before the internal wave acoustic survey. There are large scale variations in the upper 20 meters of the water column, and the lower water column appears to have fewer instabilities than the profile from MOCNESS \#9. 
in the material properties of the animals (density and sound speed contrasts) or the animal behavior itself (non-broadside orientations) could cause this (Chapter Two discusses the latter effect).

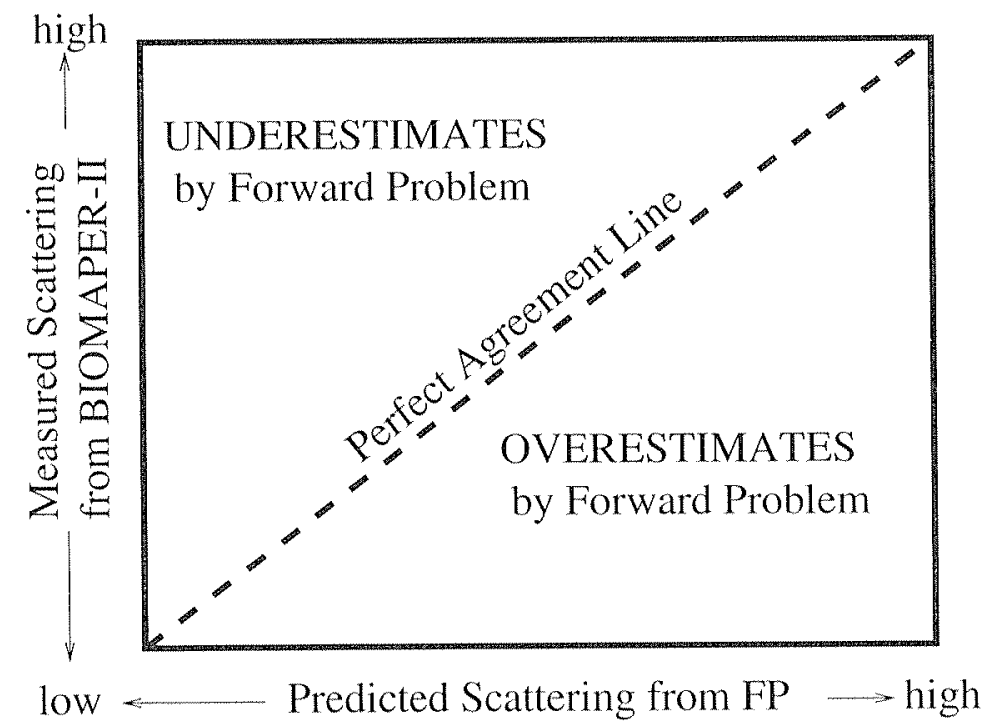

Figure 4-19: Schematic explaining the cause of errors in the plots showing the results of the Forward Problem calculations. If our model and data were perfect, all FP outputs would lie along the diagonal line. If points appear above the diagonal line, then these are "underestimates" of the scattering by the FP calculations. If points lie below the diagonal line, then they are "overestimates".

If the measured values are larger than the predicted values, then this is an "underestimate" of the FP calculation. There are several reasons why this would occur: the net tow is under sampling the actual zooplankton that are in the water column; the acoustical scattering models predict values that are smaller than the actual scattering from an animal; and other scattering processes that are not being modeled are occurring in the water column.

The FP calculation was performed accounting for scattering from biological scatterers only, this is the method that previous studies have used (Wiebe et al., 1996). The FP calculation for MOCNESS \#9 has both over- and under-estimates from the predicted scattering models (Figure 4-20). Overall, the predicted and measured values agree fairly well, however nets \# 3,4, and 5 are slight overestimates by the scattering models of the measured values of volume scattering. Nets \# 6 and 8 show 
underestimates by the scattering models. Net \#7 shows a good agreement with the predicted and measured values of volume scattering. If the scattering contributions are examined on a taxonomic basis, the dominant scatterers are salps, euphausiids, amphipods and parts of siphonophores (nectophores and pneumatophores) (Figure 4-21). The other animals (particularly the abundant copepods) contribute little to the overall predicted scattering.

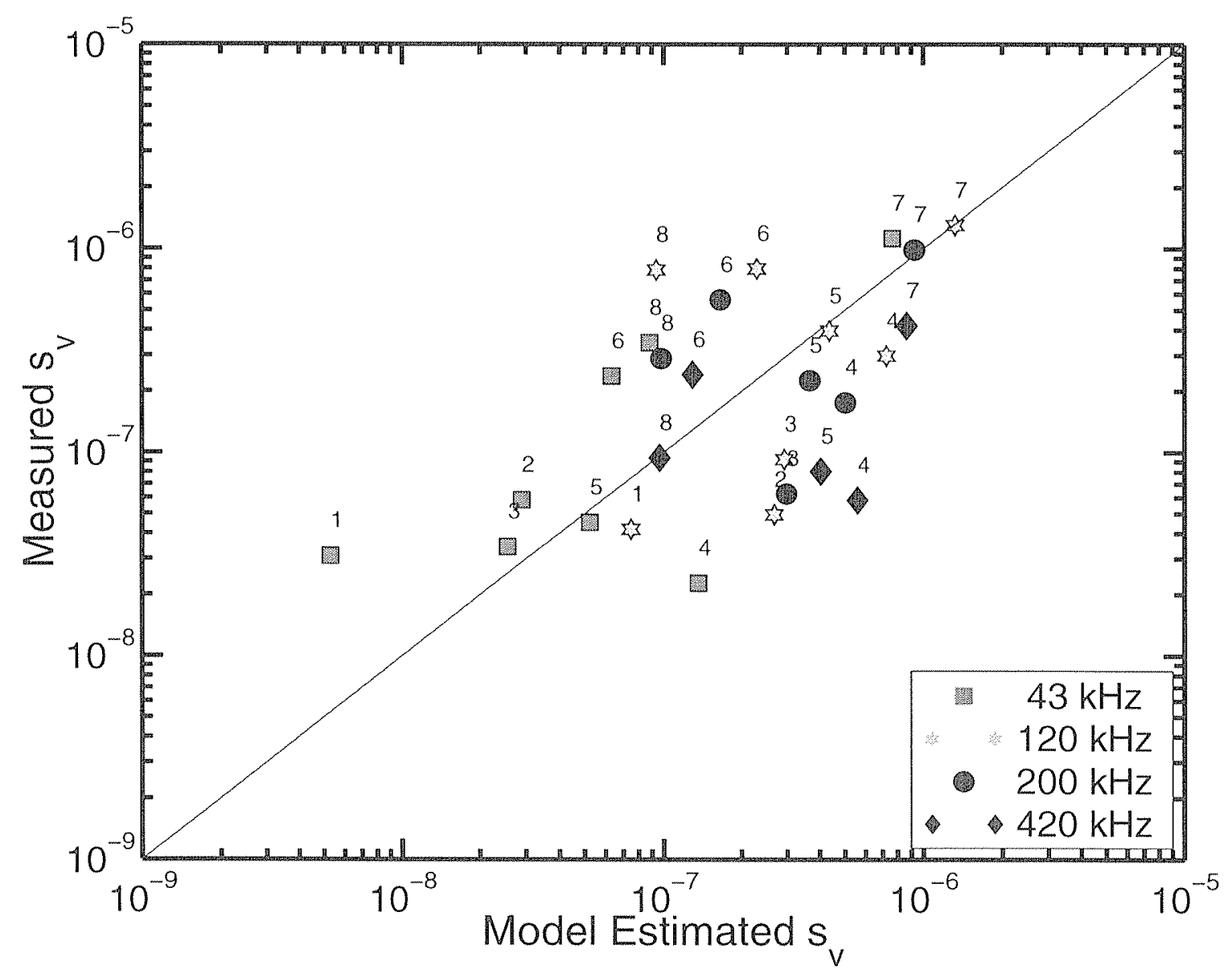

Figure 4-20: Forward Problem calculations for scattering at each frequency (symbol color and shape) and net number (number above each symbol) for MOCNESS \#9. Predictions from nets \#3-5 tend to overestimate the scattering, while most of the other predictions were underestimates. The data from net \#7 fall very close to the measured values.

Given that previous studies using these scattering models have achieved fairly accurate predictions of scattering (Wiebe et al., 1996), the most likely cause of our FP underestimates of the scattering is that other scattering processes are occurring 

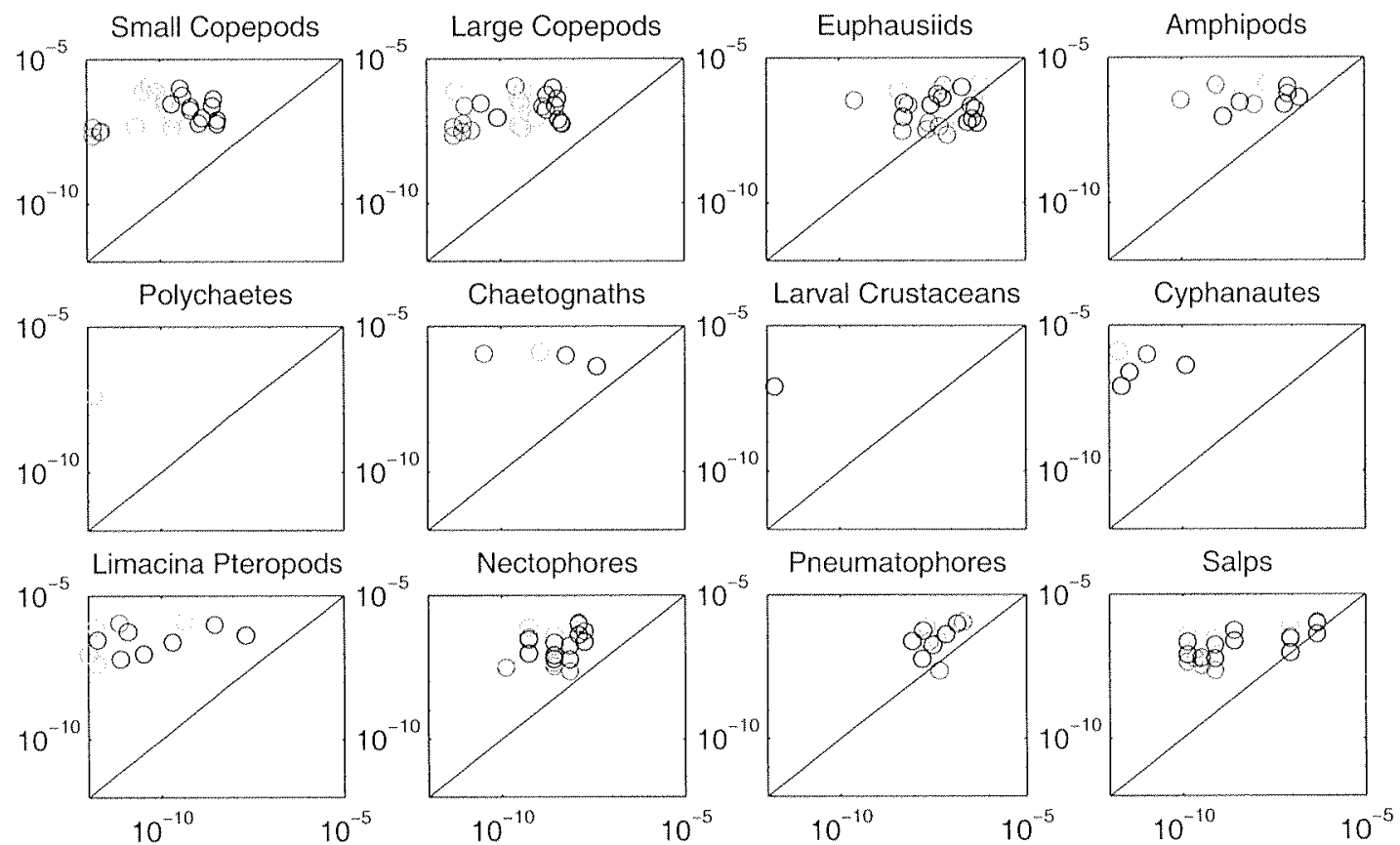

Figure 4-21: Forward problem calculations for MOCNESS \#9 broken down for different taxonomic types. The horizontal axis in each plot is the predicted $s_{v}$, while the vertical axis is the measured $s_{v}$ by BIOMAPER-II. The data are expected to lie above the vertical line since any values on or below the line mean that all of the measured scattering is accounted for by that animal. Salps, euphausiids, amphipods, and siphonophore nectophores were the largest predicted scatterers. 
in the ocean that are not being modeled. Temperature and salinity microstructure has been proposed as a source of acoustic scattering for many years (Batchelor, 1959), and field observations from the BIOMAPER-II system suggest that many scattering features are associated with hydrographic gradients in the water column. With the recent publication of a scattering model for microstructure, the data set from the October cruise is ideal to examine the possibility that scattering from microstructure may be detectable and identifiable by multiple frequency acoustic data.

\section{Prediction of $\epsilon$ using Thorpe lengths}

As discussed previously, in order to use the acoustic scattering model for microstructure, the values of the dissipation rate of turbulent kinetic energy $(\epsilon)$ and temperature variance $(\chi)$ must be found. Since direct measurements were not made of these parameters, they must be estimated. A simple method to estimate $\epsilon$ is the use of instabilities that are found in the density profile of the water column (Figure 4-22). From the vertical displacements of water parcels needed to stabilize the density profile, Thorpe lengths (Figure 4-22) were found by averaging $L_{T}$ over fourteen different depth bins, selected by eye, that separated regions of high and low variability in temperature, salinity, or density.
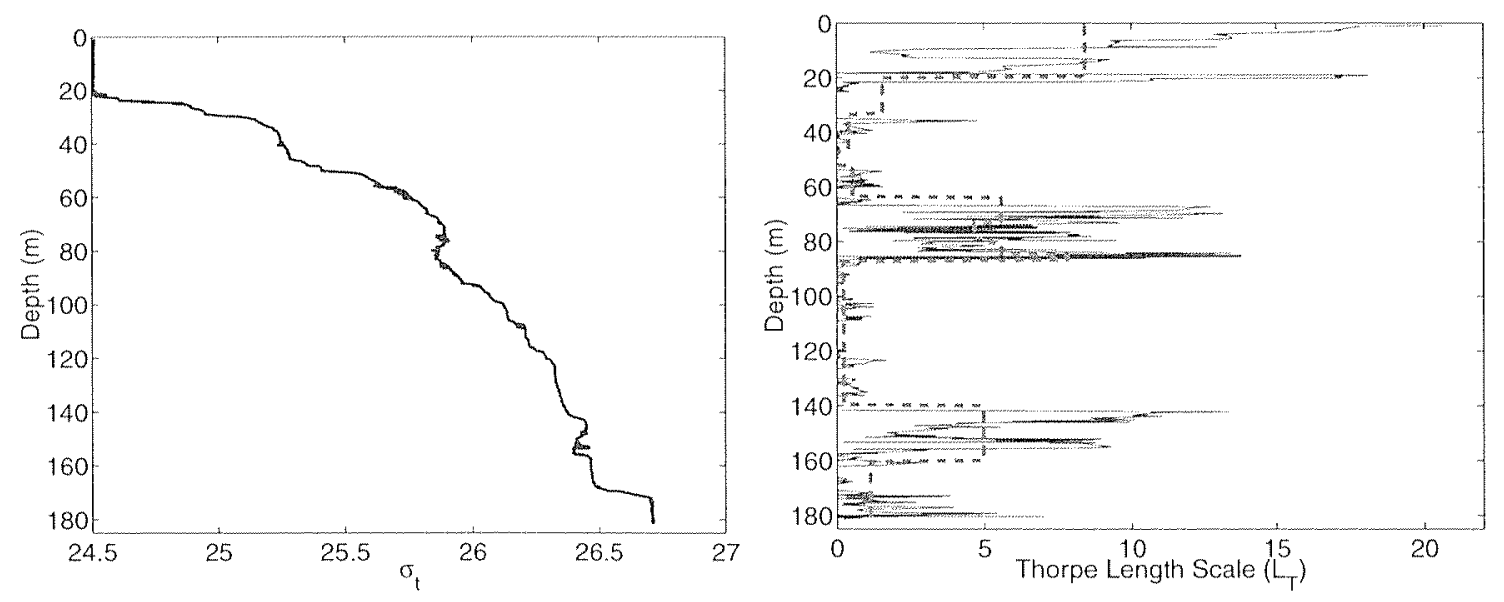

Figure 4-22: Density profile from MOCNESS \#9 (left) and the calculated Thorpe lengths (right). The dashed line is the mean $L_{T}$ for various depth bins selected by eye. 
Once the Thorpe lengths have been estimated, values for $\epsilon$ and $\chi$ can be found using equations 4.8, 4.9, 4.10, and 4.11. The resulting estimates (Figure 4-23) show larger values of both $\epsilon$ and $\chi$ in the near-surface region (0-20 meters) as well as in two midwater depth ranges (approximately 60-90 and 140-160 meters). The range of values (about five orders of magnitude) is quite large, however not atypical for estimates of $\epsilon$ and $\chi$. The estimates of $\epsilon$ and $\chi$ are reasonable (but slightly larger) than values that have been measured in regions near the continental shelf (Sandstrom et al., 1989; Rehmann and Duda, 2000). It is extremely likely that our method overestimates these parameters as a result of using the MOCNESS salinity and temperature data with the poor vertical resolution. It is also possible though that the internal waves propagating through our study region were causing very high levels of turbulence.
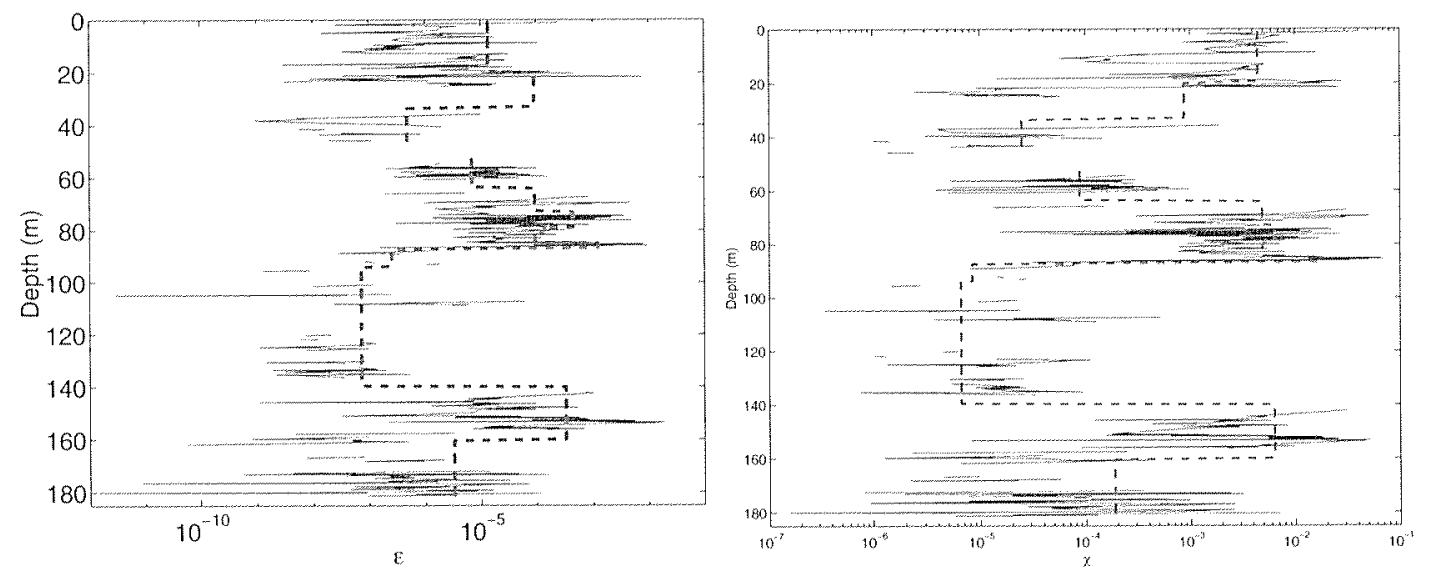

Figure 4-23: Calculated values of $\epsilon$ (left) and $\chi$ (right) from hydrographic information from MOCNESS \#9 using equations 4.8,4.9,4.10, and 4.11. Dashed lines are the mean value for the depth bins selected for the Thorpe length analysis.

Once $\epsilon$ and $\chi$ are known, they may be used to predict the acoustic scattering contributions from microstructure. The microstructure scattering model can be used to find the values of $s_{v}$ for each of the frequencies that BIOMAPER-II uses (the $1 \mathrm{MHz}$ data are not analyzed due to the limited depth range). Levels of scattering comparable to those from dense assemblages of copepods and euphausiids are predicted in certain regions of the water column by the microstructure scattering model (Figure 4-24). These high scattering regions correspond directly to the areas of the water column 
with large estimates of $\epsilon$ and $\chi$ which, in turn, are the regions with large Thorpe length scales.

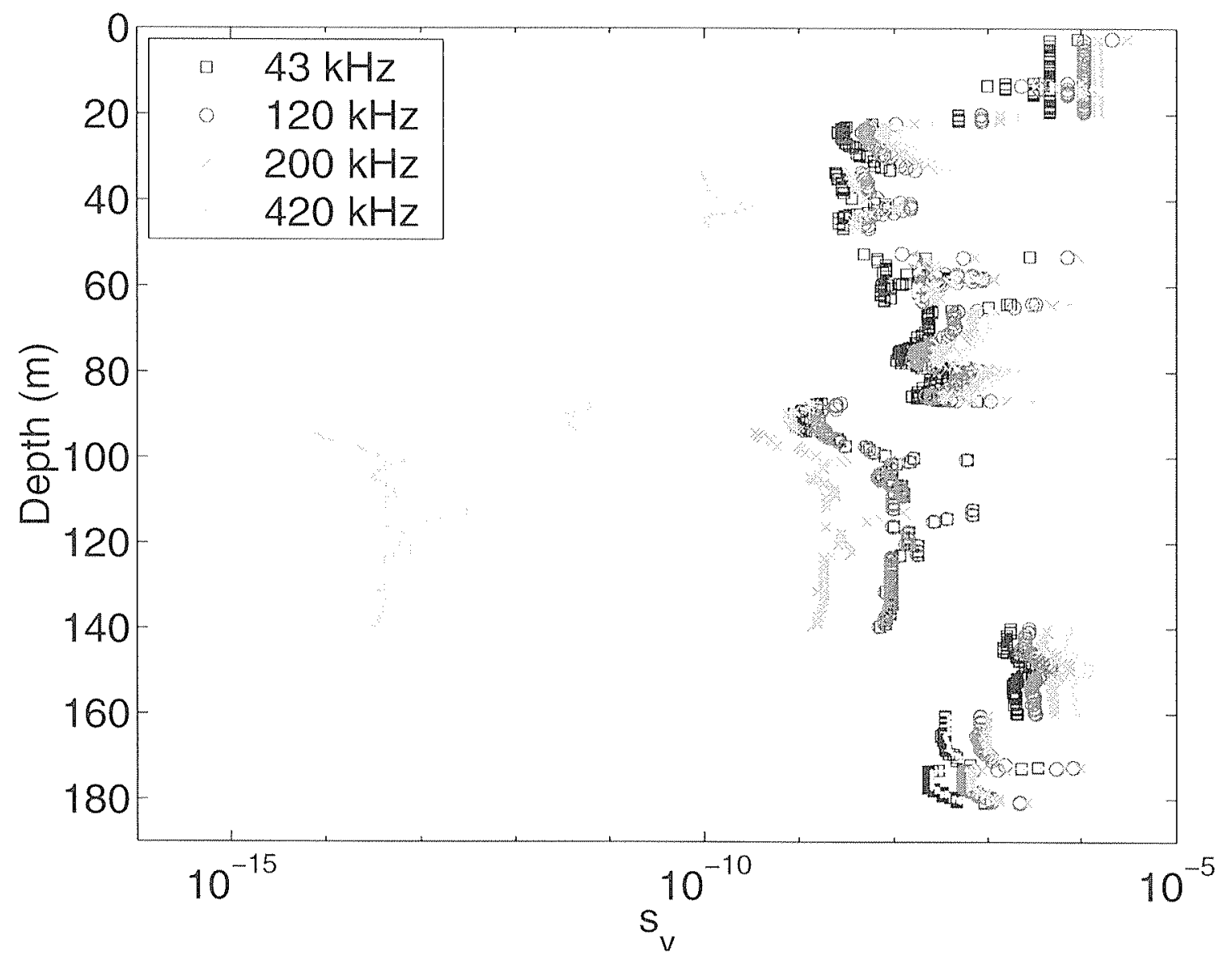

Figure 4-24: Predicted levels of volume scattering from Seim's model and the hydrographic information from MOCNESS \#9. There is increased scattering from microstructure at the surface and the lower water depths which correspond to regions that appeared to be well mixed in the density profile. It should be noted that the largest amount of scattering is not always at the same frequency which suggests that the length scale of the microstructure or turbulence is varying throughout the water column as well. These values of volume scattering are likely to be too large since they would account for all of the scattering that was measured in the water column, this is attributable to our method of estimating $\epsilon$ which is not ideal.

The predictions from the theoretical microstructure scattering model are too large for some regions of the water column (0-20, 60-90, 140-160 meters depth) because these values would account for all of the measured scattering without including any scattering from zooplankton. An interesting feature is that the scattering from the highest frequency $(420 \mathrm{kHz}$ ) is not always the lowest amplitude compared to the other 
frequencies. An example theoretical scattering spectrum predicted by Seim's model for a particular value of $\epsilon$ shows a mid-frequency peak (Figure 4-25). In the water column however, the length scales of turbulent features (Thorpe lengths, temperature, salinity, and density variability) change with depth; thereby changing the length scale of regions with different indices of refraction. These changes in refraction index are responsible for acoustic scattering (in our model), therefore changes in their size will affect how much energy different acoustic frequencies will scatter.

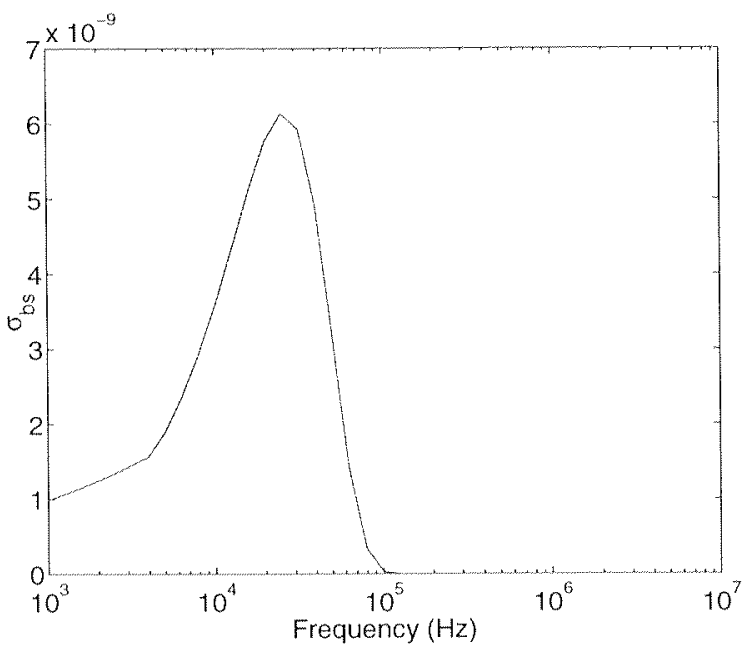

Figure 4-25: Volume scattering predicted by Seim's model for a particular point in the water column for MOCNESS \#9. This figure is meant to qualitatively illustrate the general shape of scattering spectra and show that the peak value is dependent on the size of the Bragg wavelength and the index of refraction variations. Therefore for different scales of microstructure (and index of refraction variation), this curve may be shifted to the left or right which would cause the scattering spectra to appear as increasing, decreasing or peaking depending on the acoustic frequencies (and scale of microstructure) used.

In the theoretical model, the size of the acoustic Bragg wavenumber relative to the length scale of refraction index variations determines whether the scattering spectra has a positive or negative slope. In different regions of microstructure, the scattering spectra will be shifted to the left or right depending on the scales of the index of refraction variances and the Bragg wavenumber. This allows the different acoustic frequencies to have different scattering values which are microstructure (and depth) dependent. It is possible that the differences in scattering strength from multiple frequencies may be able to be analyzed for information regarding the length scale of 
the physical scattering process. This is an entertaining theory, but exploration of it is beyond the scope of this thesis.

\section{Inversion of Acoustic Data for $\epsilon$}

In order to verify that our estimates of $\epsilon$ and the scattering from microstructure are reasonable, a different method to estimate $\epsilon$ is needed. One way to do this is to run the theoretical scattering model for a wide range of values of $\epsilon\left(\epsilon=10^{-10}-10^{-3}\right)$. This produces plots of $\sigma_{b s}$ or $S_{v}$ versus depth versus $\epsilon$ and these plots can be made for a specific acoustic frequency, such as those used by BIOMAPER-II (Figure 4-26).
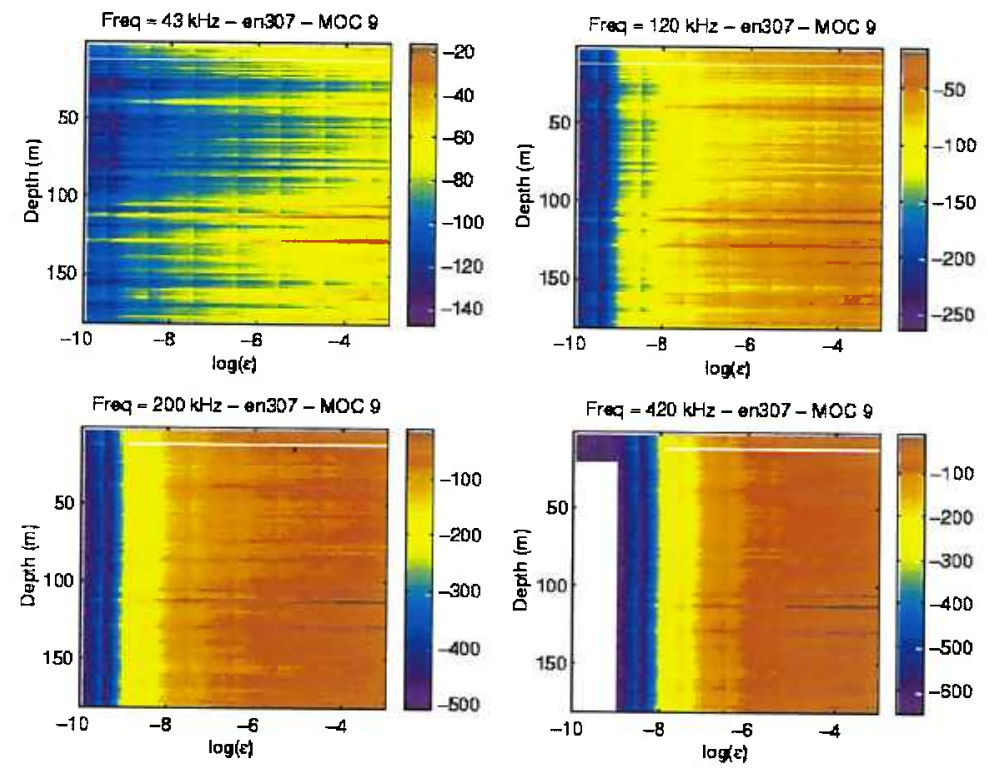

Figure 4-26: Theoretical values of volume scattering strength from the Seim model for the four frequencies used by BIOMAPER-II and a wide range of $\epsilon$ values. These data are used in the inversion of the "leftover" scattering data (once the biological caused scattering is accounted) to provide predictions of $\epsilon$ in the water column. The $S_{v}$ colorbars have different scales in each sub-plot.

If the differences between the measured and estimated scattering values from the Forward Problem calculations (Figure 4-27) are assumed to be contributions to the scattering from microstructure, then this value of scattering can be inverted (using the output of the theoretical microstructure scattering model) for values of $\epsilon$, the dissipation rate of turbulent kinetic energy (Figure 4-28). This method can only be used for regions where the measured scattering is larger than the estimated scattering, 
since if the measured scattering was smaller than the estimates then that implies that our zooplankton scattering models are inaccurate (to some extent) or, even more unlikely, that microstructure is somehow absorbing sound energy.

When estimates of $\epsilon$ are found for the depth ranges that correspond to the MOCNESS tows, there were low values $\left(10^{-9}\right)$ near the bottom, and larger values $\left(10^{-5}-\right.$ $10^{-4}$ ) in the near surface. The shallower region corresponds to the depths that the internal wave was seen in the first acoustic transect. For comparison, Rehmann and Duda (2000) report values of $\chi$ ranging from $10^{-9}-10^{-5}$ on the New England shelf south of Cape Cod, MA, while Hebert et al. (1992) report a similar range of values for $\epsilon$ for a study of an internal wave in the Pacific Equatorial Undercurrent. The dissipation rate of temperature variance should be of the same order of magnitude in our calculations as $\epsilon$ except in regions with large density gradients. Although the locations of these studies are not the same as our internal wave survey, similar wave and mixing processes occur at both locations so similar values for $\epsilon$ would also occur.

\section{Modifications to Forward Problem Calculations}

The most likely errors in our Forward Problem calculations are from the acoustic scattering models that are used. The model describing scattering from microstructure is recently published (Seim, 1999) and has been tested with only a few field-collected data sets. Further testing of this model is needed, but is beyond the scope of this thesis. The zooplankton scattering models have been used previously in the analysis of field-collected data (Wiebe et al., 1996, 1997; Greene et al., 1998), however there are many variables used in these models that are inadequately understood (animal behavior and orientation, material properties of the zooplankton). Net tow information from MOCNESS systems have been used for several decades and sampling errors from it are likely limited to zooplankton avoidance of the net and extrusion through the mesh. These artifacts are believed to be small, therefore modifications to the Forward Problem will focus on changes in the zooplankton scattering models used.

Fluid-like animals, which constitute the majority of zooplankton taxa that are en- 


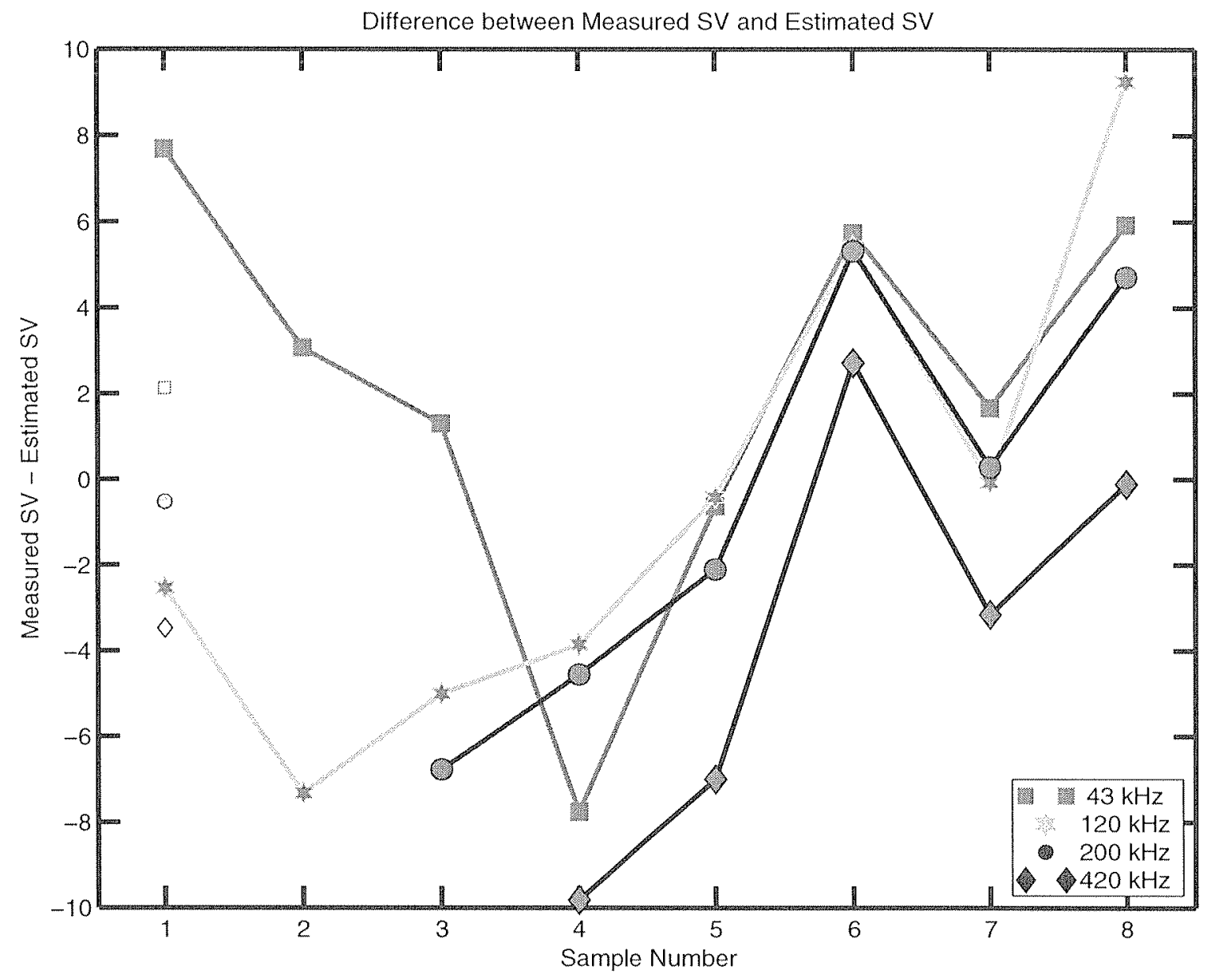

Figure 4-27: Errors in the measured and predicted levels of volume scattering for MOCNESS \#9. The vertical axis is the difference between the measured volume scattering strength (from BIOMAPER-II data) and the predicted level of scattering (from theoretical scattering models and MOCNESS zooplankton and CTD data). The volumes are determined by where the MOCNESS nets were opened and closed. The horizontal axis is MOCNESS net number where \#1 is the deepest net and \#8 is the near-surface tow. Values above 0 are underestimates and values less than 0 are overestimates by the FP predictions. The underestimated values are used in the inversion of acoustic scattering data for values of $\epsilon$. Mean values for each frequency are on the left. 


\section{Data from MOC $9 t(z)$ and $s(z)$ profiles}

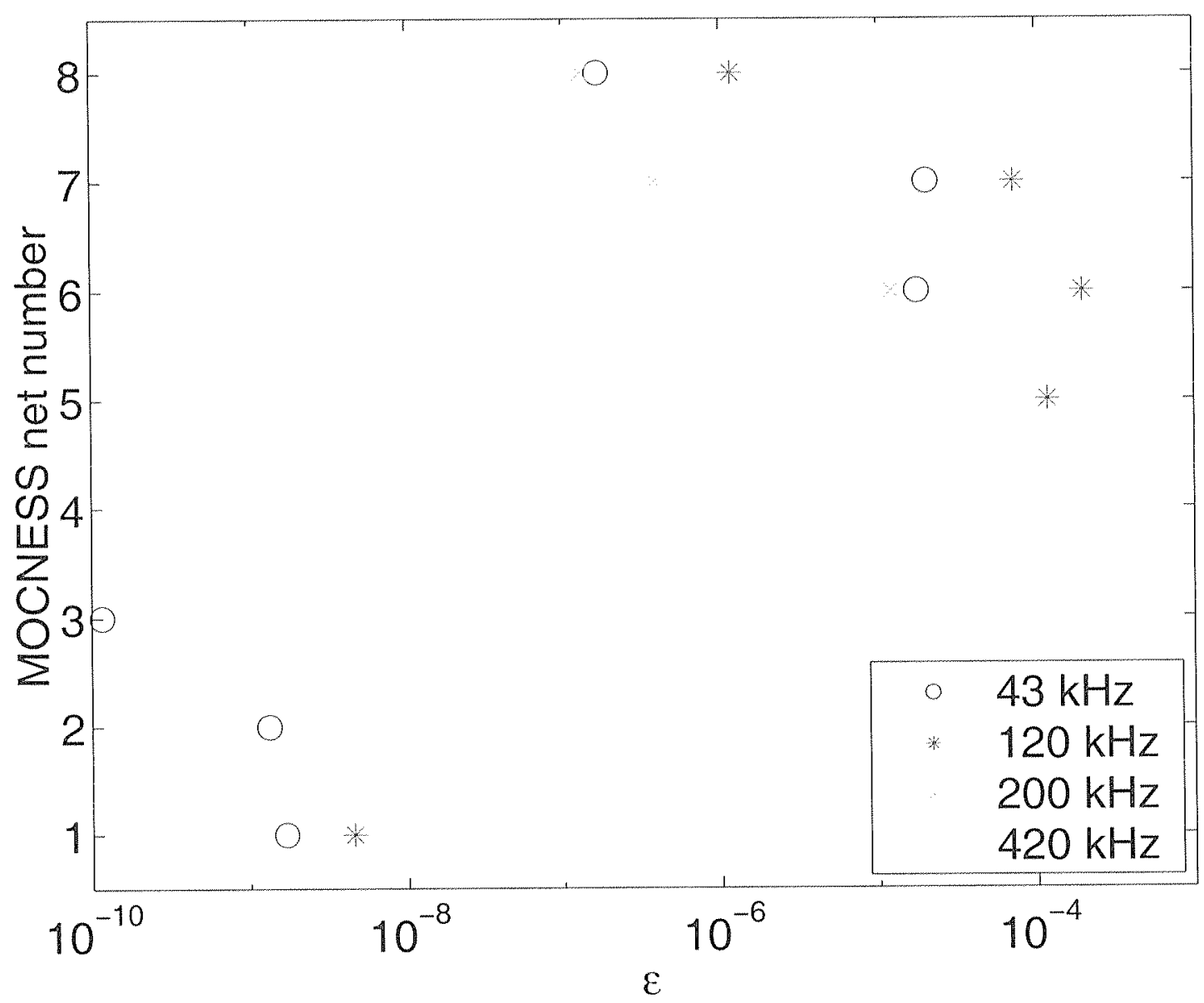

Figure 4-28: Estimates of $\epsilon$ from the remaining measured acoustic scattering once biological sources have been accounted for. Large values of $\epsilon$ are found in the surface layer and are of the order of dissipation rates for an internal wave. Smaller values are found in the deeper waters where the internal wave was not seen to be propagating and are typical for open ocean turbulence. 
countered in the Gulf of Maine and Georges Bank region, are modeled as a weakly scattering, bent, fluid cylinder. A DWBA (Distorted Wave Born Approximation) method (Stanton et al., 1993a; Chu et al., 1993) is used in this analysis, with the input parameters being $g, h$, the length to width ratio of the animal, and the orientation of the cylinder relative to the acoustic wavefront. The Forward Problem model previously used an average over all angles of orientation $\left(0-360^{\circ}\right)$, however if known orientation data are used for euphausiids (Sameoto, 1980; McGehee et al., 1998) then a more realistic range of angles is $290^{\circ} \pm 20^{\circ}$ where $270^{\circ}$ is broadside orientation, so the euphausiid posture is slightly head-up.

Very little is known about the general orientation of other animals that use the fluid-like model; so an average over all orientations will continue to be used for these animals, as well as other fluid-like zooplankton. Although preliminary results from the analysis of VPR data indicate that copepods may tend to orient themselves vertically in the water column (Benfield et al., 2000). To actually implement these models in the FP calculations, a look-up table of values of RTS (Reduced Target Strength) and $\mathrm{ka}$ is used. RTS normalizes the TS by the $\log$ of the square of a dimension of the animal, in our calculations the length of the animal $(L)$ is used (since $L$ is measured in the silhouette photograph analysis of the MOCNESS tow)

$$
R T S=T S-10 \log \left(L^{2}\right)
$$

There are slight differences in the look-up tables that are used for the fluid-like zooplankton. Because of differences in the length to width ratios of different animal types, the fluid-like zooplankton are split into four modeling groups: euphausiids and decapod shrimp (Figure 4-29a), chaetognath and polychaetes (Figure 4-29b), amphipods (Figure 4-29c), copepods and other crustaceans (Figure 4-29d). The same density and sound speed contrasts are used $(g=1.0357$ and $h=1.0279)$ in all cases (Foote, 1990) primarily because there is no information available about the material properties for animals other than euphausiids.

The "look-up" table format is also used for scattering from siphonophore pneu- 
(a)

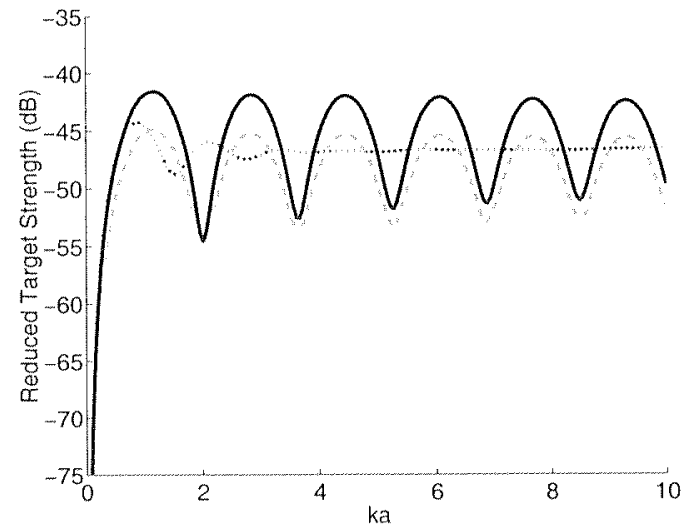

(c)

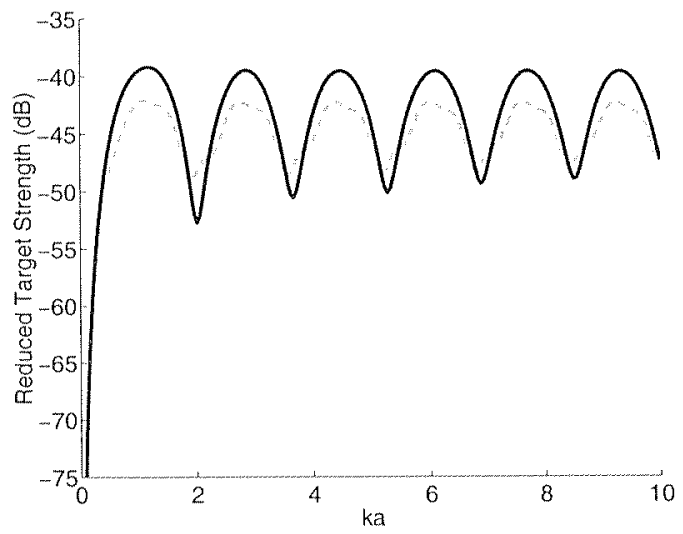

(b)

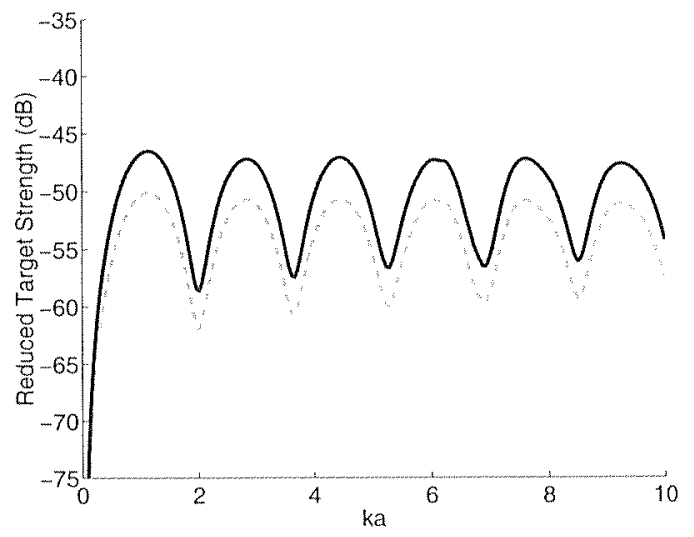

(d)

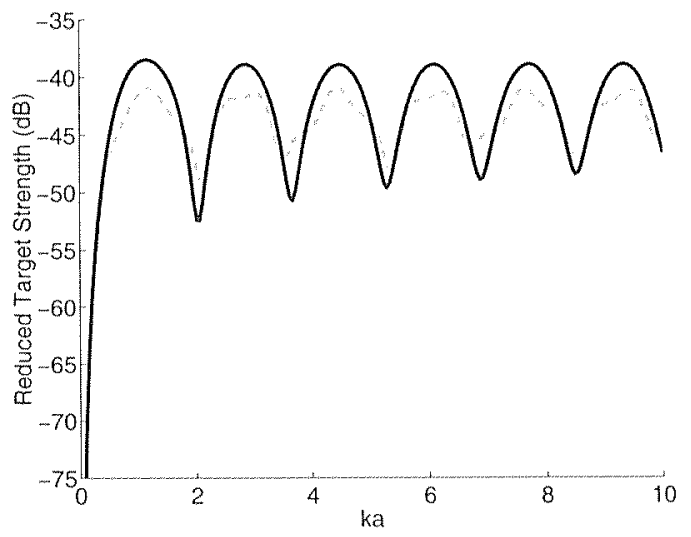

Figure 4-29: Scattering models used for fluid-like animals (euphausiids and decapod shrimp (a), chaetognaths and polychaetes (b), amphipods (c), and copepods and other crustaceans (d)) in the Forward Problem calculations. The only difference between the four models is in the length-towidth ratio of the animal. Solid lines represent "realistic" animal orientations, dashed lines are for a uniform animal orientation (averaged over $360^{\circ}$, and the dotted line in (a) is the original fluid-like scattering model used in previous Forward Problem calculations. 
matophores and any other animals whose scattering is dominated by a gas-inclusion. The scattering model used is from Anderson (1950) which describes scattering from a fluid sphere. As discussed in Chapter Three, the scattering spectra has a peak near the resonance frequency of the gas bubble (Figure 4-30). The only modification to the scattering model used is to include a higher-resolution (in ka space) look-up table. The scattering model for an elastic-shelled scatterer is also shown, although no modifications were made to this model which has been used previously. Appendix $\mathrm{C}$ contains further information on the scattering models used.

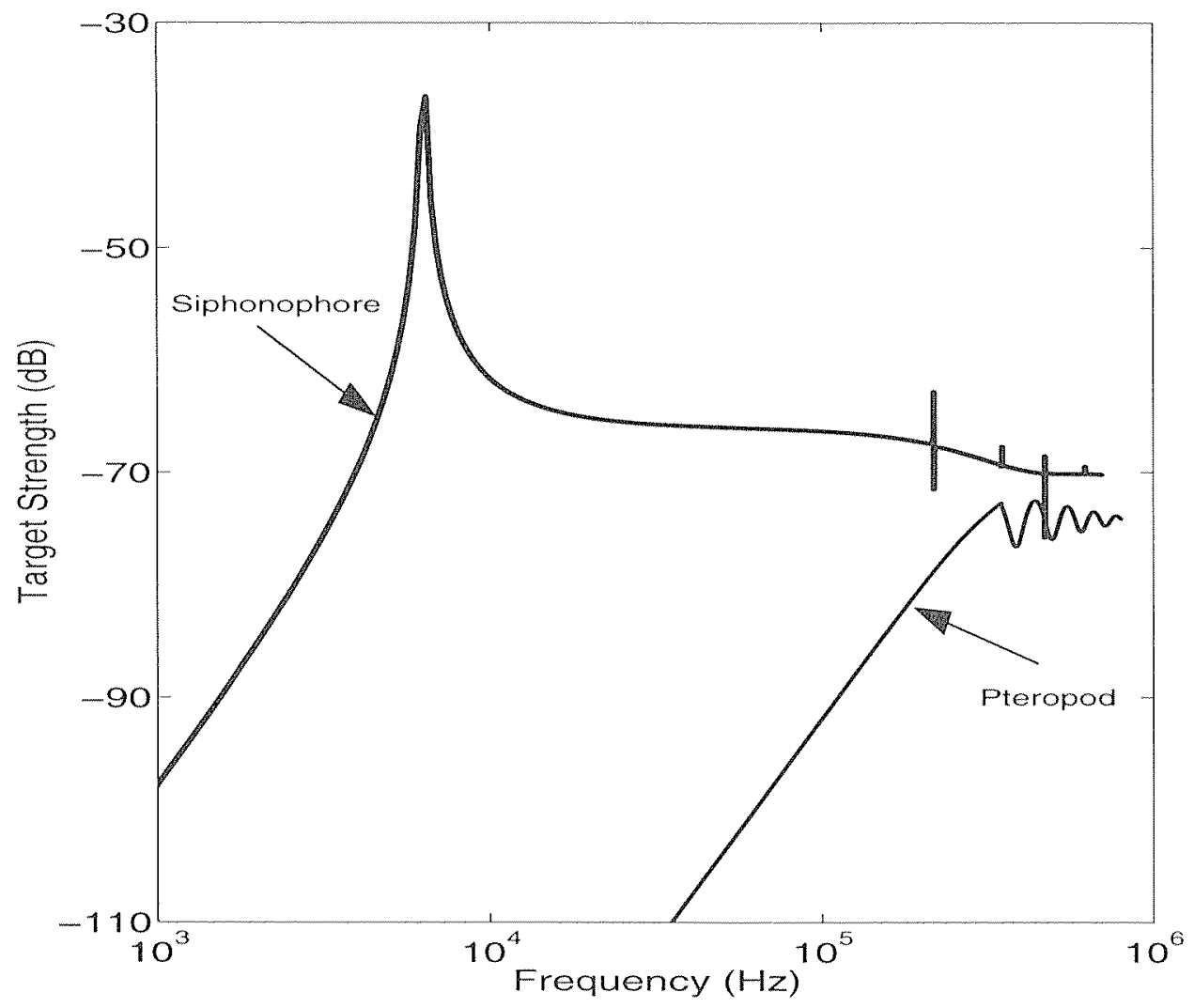

Figure 4-30: Scattering model used for the pneumatophores of gas-bearing organisms such as siphonophores. Other gelatinous organisms (salps, siphonophore bracts) were modeled as fluid-like scatterers. Also shown is the scattering spectra for an elastic-shelled animal such as a pteropod.

With the revised zooplankton scattering models in place and the inclusion of the microstructure scattering model, the FP calculations were repeated to assess the contributions that biological and physical processes make to the acoustic scattering. 
The modified FP predictions show more over-estimates than under-estimates (Figure 4-31), which is not surprising since an additional scattering process (microstructure) is included. The composition of the predicted scattering level demonstrates that microstructure can contribute a substantial amount to the overall scattering (Figure 4-32). Other changes due to the modified zooplankton scattering models are an increase in the contribution from copepods (particularly the larger ones) and changes in the errors associated with each frequency and net (Figure 4-33).

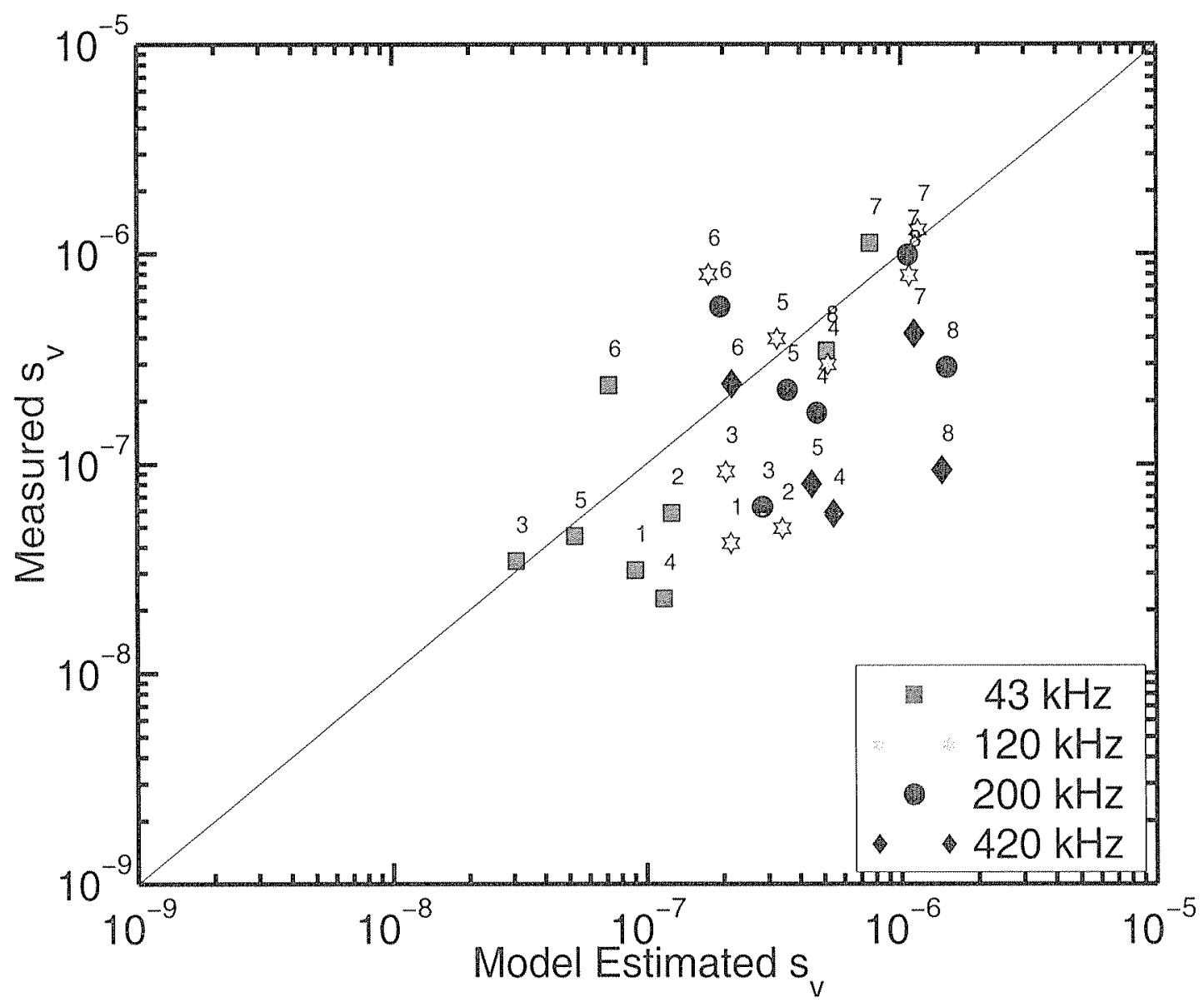

Figure 4-31: Forward Problem calculations for MOCNESS \#9 including microstructure and the modified zooplankton scattering models. There are slightly more over-estimates of the scattering by the FP calculations.

To quantify the relative errors of the three methods used in the FP calculations (original zooplankton scattering models, modified zooplankton scattering models, and 

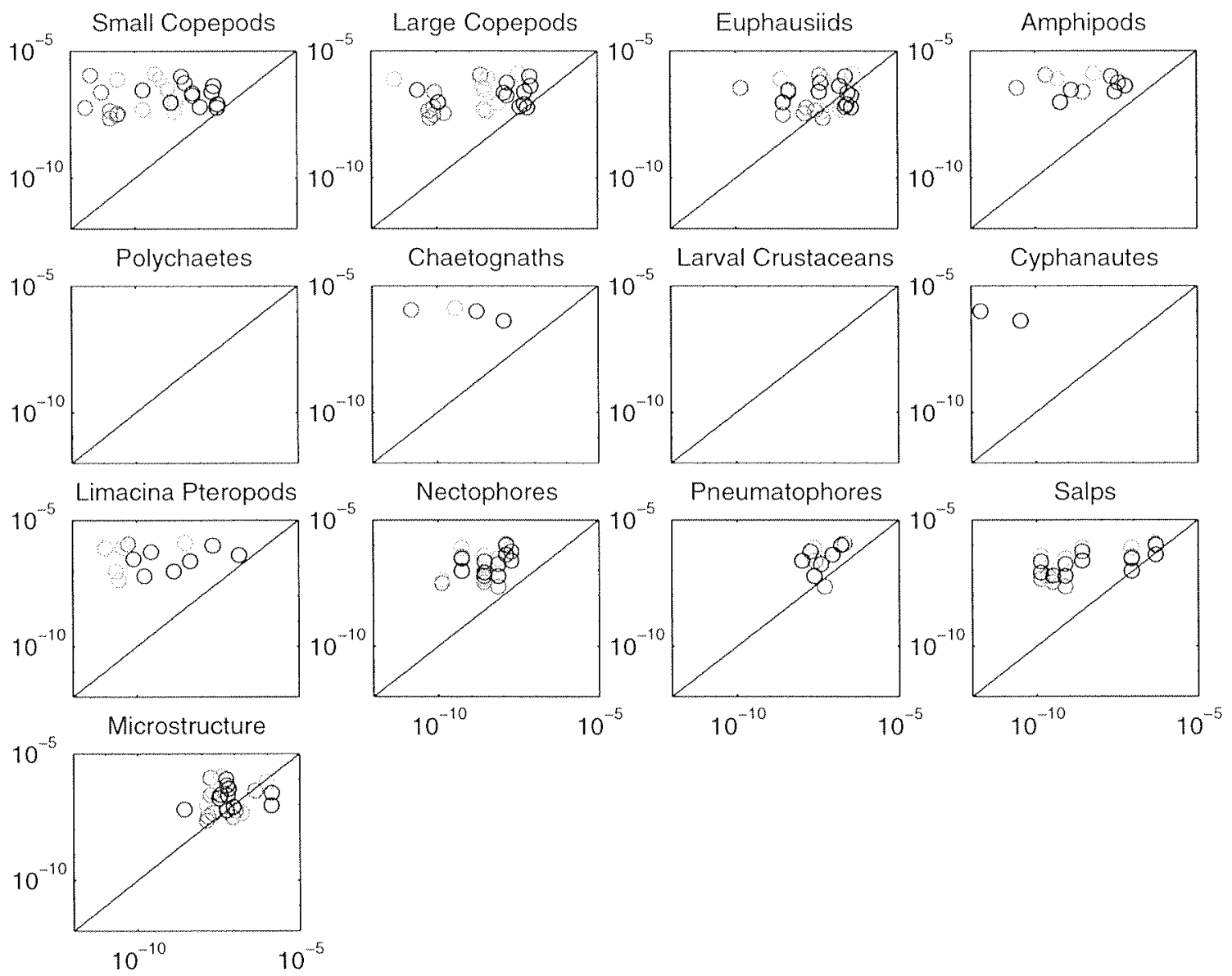

Figure 4-32: Forward problem calculations using the modified zooplankton scattering models and including microstructure contributions for MOCNESS \#9 broken down for different scattering processes. The horizontal axis in each plot is the predicted $s_{v}$, while the vertical axis is the measured $s_{v}$ by BIOMAPER-II. The data are expected to lie above the vertical line since any values on or below the line mean that all of the measured scattering is accounted for by that animal. Salps, euphausiids, large copepods, siphonophore nectophores and microstructure were the largest predicted scatterers. 


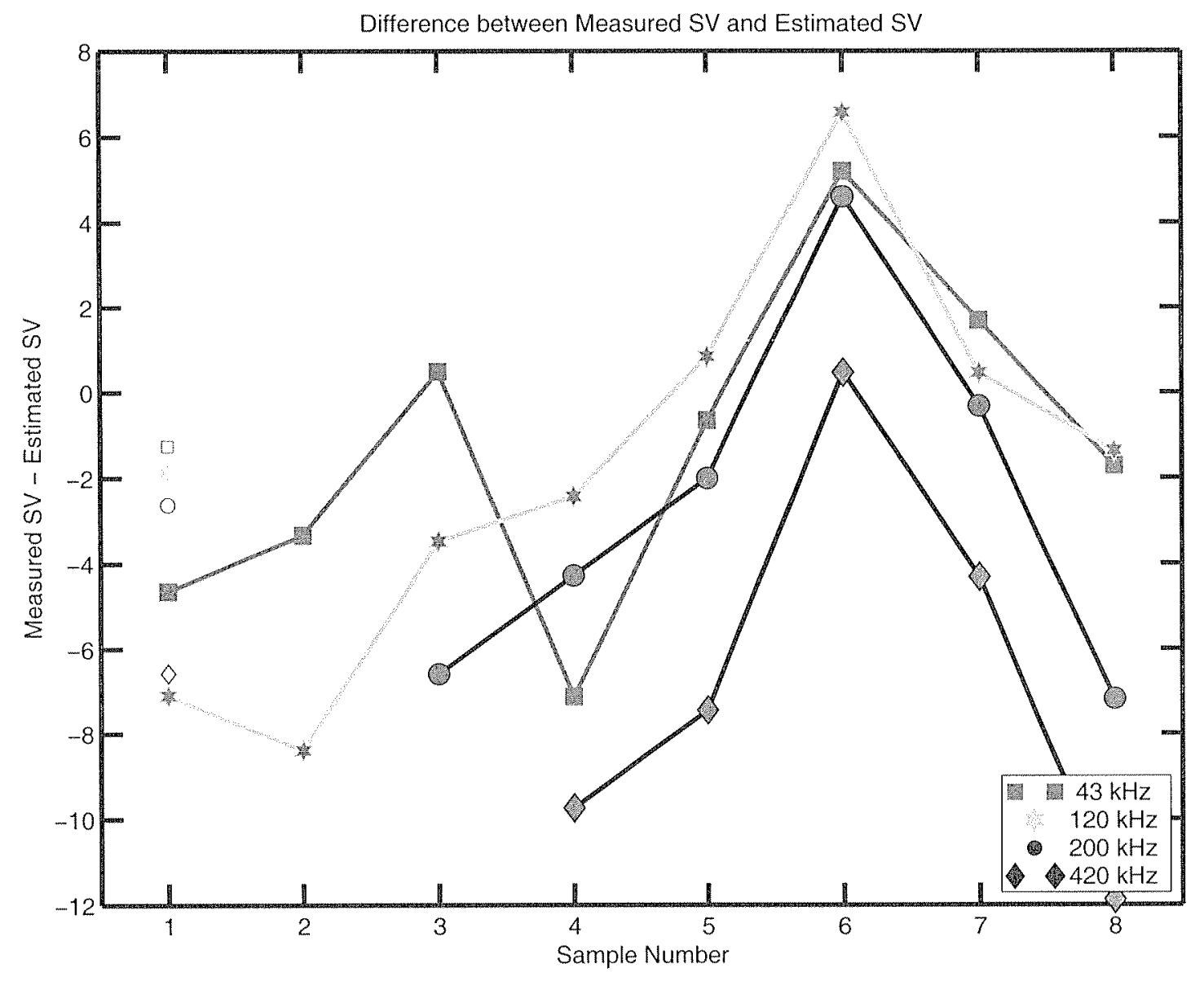

Figure 4-33: Errors in the modified Forward Problem calculations for MOCNESS \#9 including scattering contributions from microstructure and modified zooplankton scattering models. The vertical axis is the difference between the measured volume scattering strength (from BIOMAPERII data) and the predicted level of scattering (from theoretical scattering models and MOCNESS zooplankton and CTD data). The volumes are determined by where the MOCNESS nets were opened and closed. The horizontal axis is MOCNESS net number where \#1 is the deepest net and \#8 is the near-surface tow. More overestimates (negative values) are seen which is due to the large amount of scattering predicted by the microstructure scattering model, however the overall level of error is similar to that when microstructure scattering is not included. 
inclusion of microstructure scattering model), the difference between the predicted and measured values of $S_{v}$ are shown for each net and frequency for MOCNESS tow \# 9 (Figure 4-34). Generally all three methods have the same order of magnitude of error, however the first method (original zooplankton scattering models) tend to underestimate the amount of scattering while the final method (modified zooplankton scattering models and turbulence scattering models) tend to overestimate the measured amount of scattering. Errors range from $1-10 \mathrm{~dB}$.

While these data may indicate that the inclusion of microstructure as a contributor to the scattering in the ocean does not improve the accuracy of the FP calculations, we have hypothesized reasons why the turbulence values are too large, and believe that with proper measurements of the inputs into the scattering models that the overall error in the FP calculations would be reduced.

\subsubsection{Sources of Acoustic Scattering}

The net tow data provide a clear answer to the contributions that different zooplankton taxa make to the overall amount of biomass. Similarly, the relative contribution of different biological and physical sources of scattering can be made. The relative contribution of each scattering source (each animal taxa and microstructure) is found for each MOCNESS net and BIOMAPER-II frequency. The percentage contribution to the overall scattering strength is found by dividing the $\sigma_{\text {source }}$ by the overall calculated scattering prediction $s_{v}$, where

$$
s_{v}=\sum_{i=1}^{N} \sigma_{i}
$$

for $\mathrm{N}$ sources of scattering. The relative contributions were found with and without microstructure contributions.

The lower water column and surface layer were dominated by scattering from microstructure in MOCNESS tow \#9 (Figure 4-35). Euphausiids were the dominant scatterers in the mid-water depths, with siphonophore pneumatophores and 
(a)

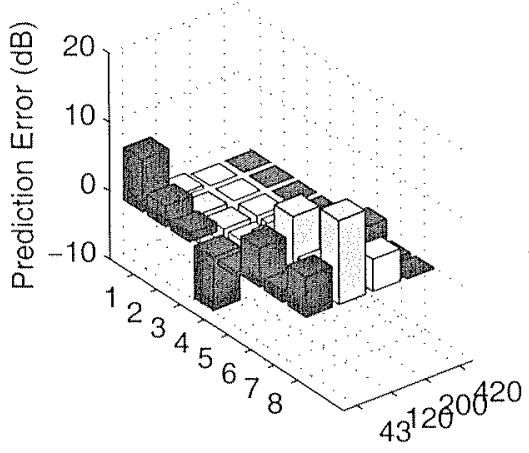

(d)

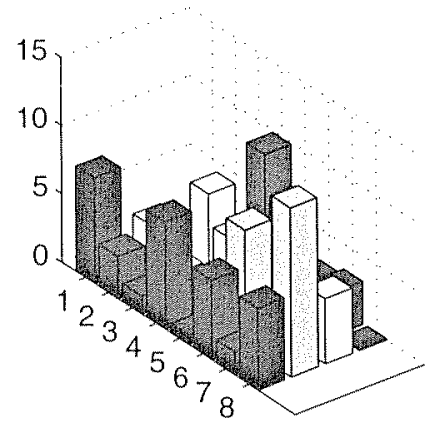

(b)

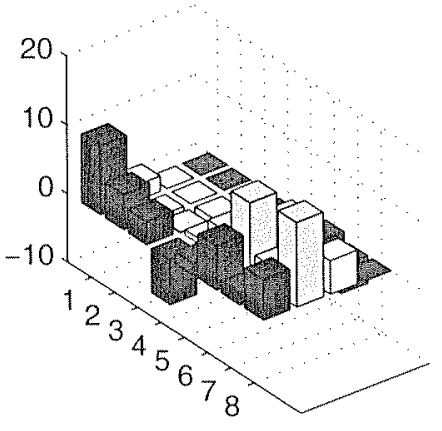

(e)

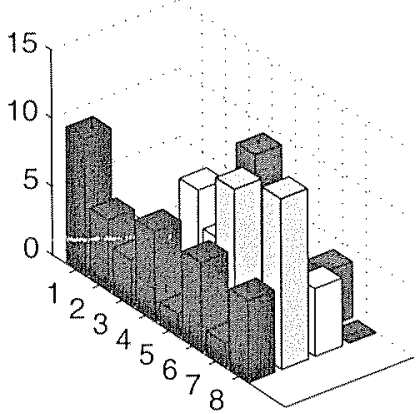

(c)

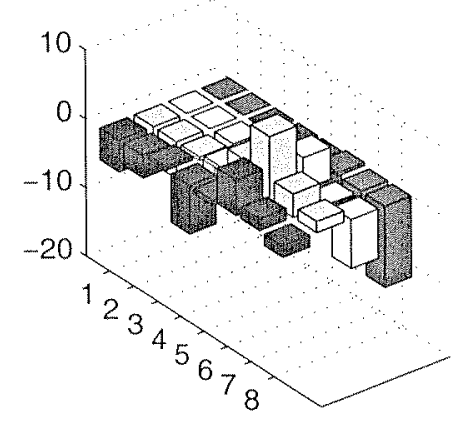

(f)

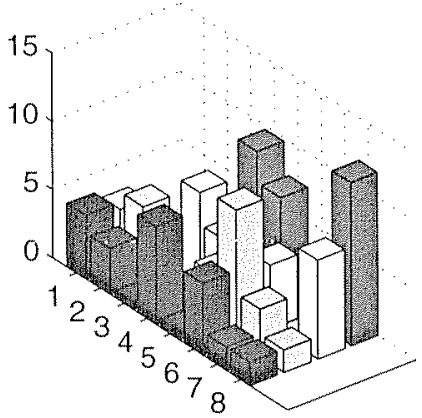

Figure 4-34: Errors in the Forward Problem calculations for MOCNESS \#9 for the original zooplankton scattering models (left column), modified zooplankton scattering models (middle column) and the modified zooplankton and microstructure models (right column). The top row are the relative errors (where positive values are underestimates and negative values are overestimates) by the FP calculations, the bottom row is the absolute value of the errors. The left-horizontal axis labels of $1-8$ represent the MOCNESS net number, and the different BIOMAPER-II frequencies (in $\mathrm{kHz}$ ) are the other horizontal axis. The modified zooplankton scattering models combined with microstructure appear to give slightly less error than the modified zooplankton scattering models alone, however if the absolute error is quantified, there is not a statistical difference between the two methods. Instead of underestimates of the measured scattering, the microstructure and modified zooplankton method overestimates the measured scattering. 
nectophores also contributing. The enormous number of salps collected in net \#7 accounted for almost all of the biomass in those nets, but the Forward Problem shows that biomass rarely is directly related to acoustic scattering. Salps are extremely weak scatterers and while outnumbering the other animals and dominating the biomass in net \# 7 , they contributed only $30 \%-60 \%$ of the total acoustic scattering predicted for this net.

If microstructure is excluded in the FP calculations (Figure 4-36), then euphausiids dominate the scattering below 40 meters depth while salps are the main contributors to the surface scattering layers. Siphonophores and assorted fluid-like animals also contribute to the scattering. A comparison between the figures with and without microstructure along with the biomass measured by the net tow (Figure 4-13) shows the errors that may occur if the contributions from microstructure are not accounted for in the FP. The net data show that peaks in biomass occur in net \#7 (due to salps) and in nets \# 3-4 (due to euphausiids). This agrees with the data from the FP calculations (except that net \# $3-5$ have a similar composition) that includes microstructure scattering contributions.

The goal of this work is to improve the ability of using acoustical scattering information to assess populations of zooplankton. A comparison between the measured scattering during MOCNESS \#9 and the predictions (at each frequency) for each net of the scattering (both with and without microstructure) show that there are still many problems with this method (Figure 4-37). It was thought that the inclusion of microstructure as a source of acoustic scattering would correct many of the errors in the FP calculations, and in some cases this is true. However, in other regions, the inclusion of contributions from microstructure produce even larger errors.

However if the relative contributions from each scattering source are identified from the FP calculations, then the percentages of scattering from physical and biological sources is known. This can be combined with the measured scattering from the BIOMAPER data to produce a depth profile of biologically-caused acoustic scattering (Figure 4-38). If physical scattering contributions are removed from the acoustic 


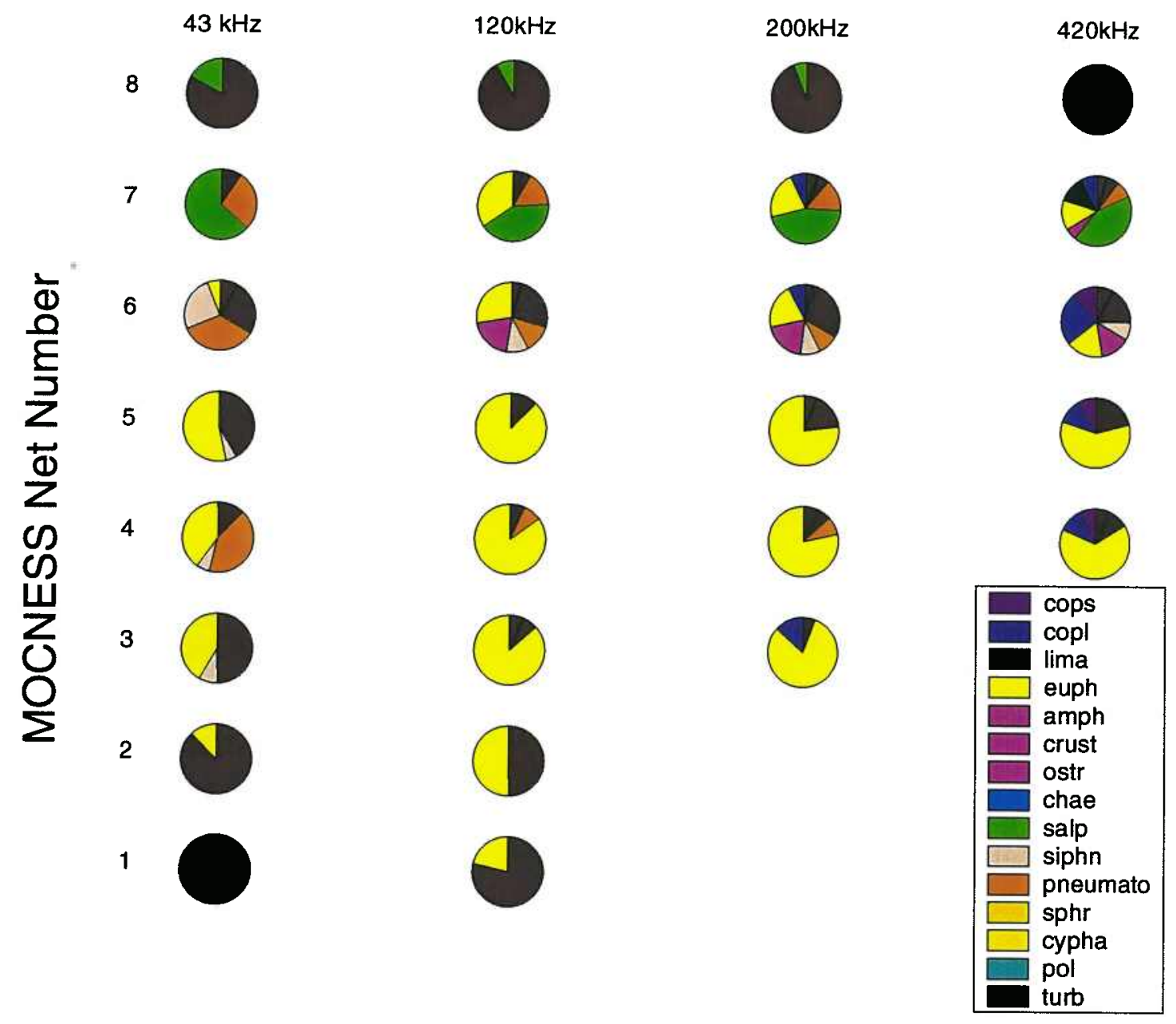

Figure 4-35: Composition of the acoustic scattering predicted by Forward Problem calculations at four BIOMAPER-II frequencies using biological and hydrographic information from MOCNESS \#9. For each BIOMAPER frequency (since scattering changes with acoustic frequency), the predicted contribution of scattering from microstructure (from MOCNESS CTD data) and each type of zooplankton (from MOCNESS net samples) is found. These data include contributions from microstructure which are largest in the surface net (\#8) and the bottom two nets (\#1-2). While salps dominate the scattering from net \#7, euphausiids are the dominant scatterers in the rest of the water column. Siphonophore pneumatophores also contribute a substantial amount of scattering in some cases. These data can be used to estimate how much of the measured scattering is from each type of scattering source, which is necessary for proper interpretation of acoustic scattering measurements in field surveys. 


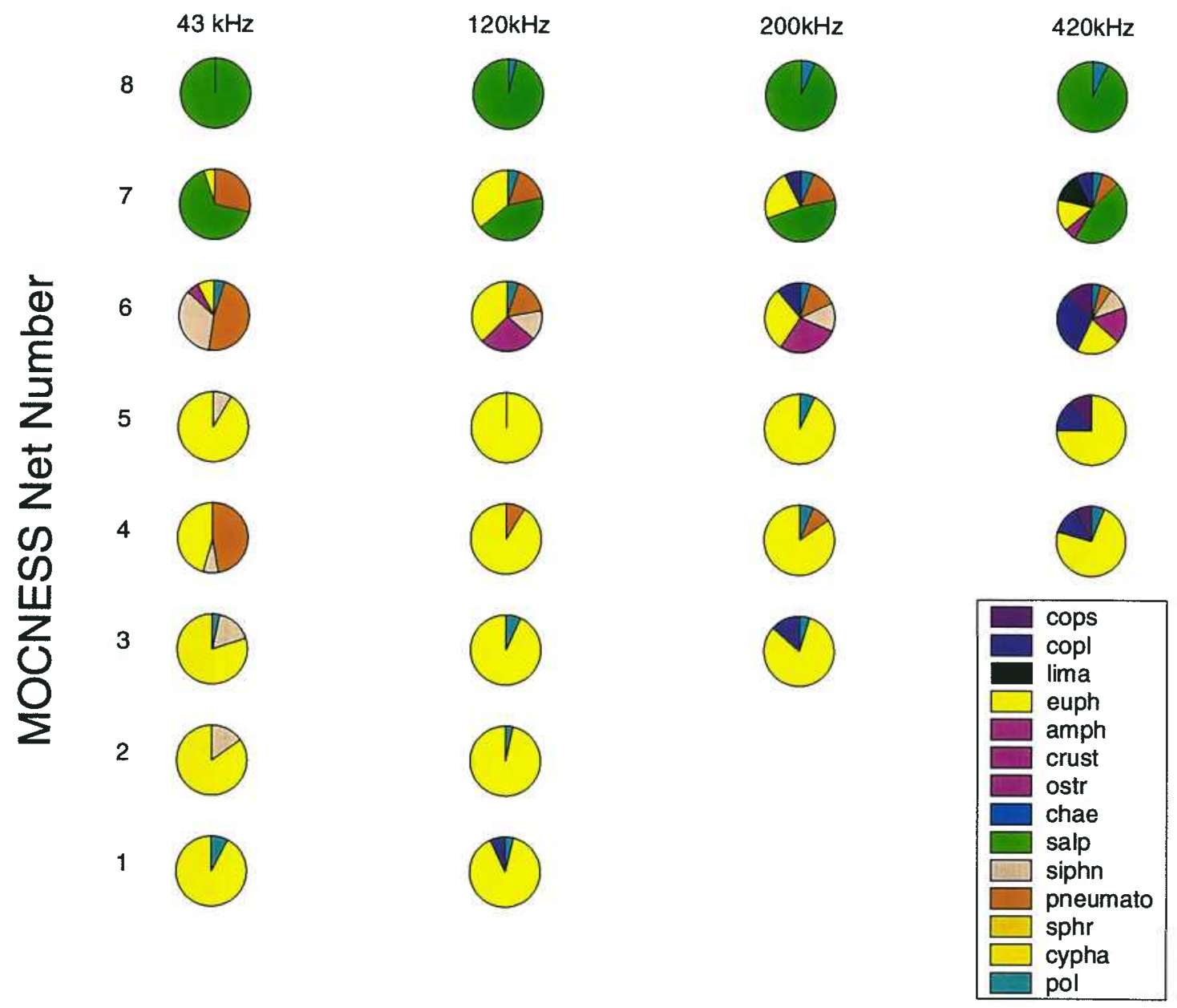

Figure 4-36: Composition of the acoustic scattering predicted by Forward Problem calculations at four BIOMAPER-II frequencies using biological and hydrographic information from MOCNESS \#9. These data do NOT include contributions from microstructure. Without microstructure, it appears that salps would dominate the upper two nets and euphausiids (and occasionally siphonophore pneumatophores) contributed the rest of the scattering. The differences between this figure and Figure 4-35 clearly show the importance of scattering contributions from microstructure, and how the neglect of this scattering process can cause errors in the interpretation of acoustic backscatter data. For example, if microstructure scattering is not included the scattering in the upper 40 meters of the water column (nets \# 7-8) would be thought to be dominated by salps, while scattering in the rest of the water column would be from euphausiids. A comparison of this figure and Figure 4-35) show that the scattering in nets $\# 1,2$, and 8 is not dominated by scattering from biological animals, rather scattering from physical processes. This type of analysis would result in more accurate interpretation of acoustic data from zooplankton field surveys. 


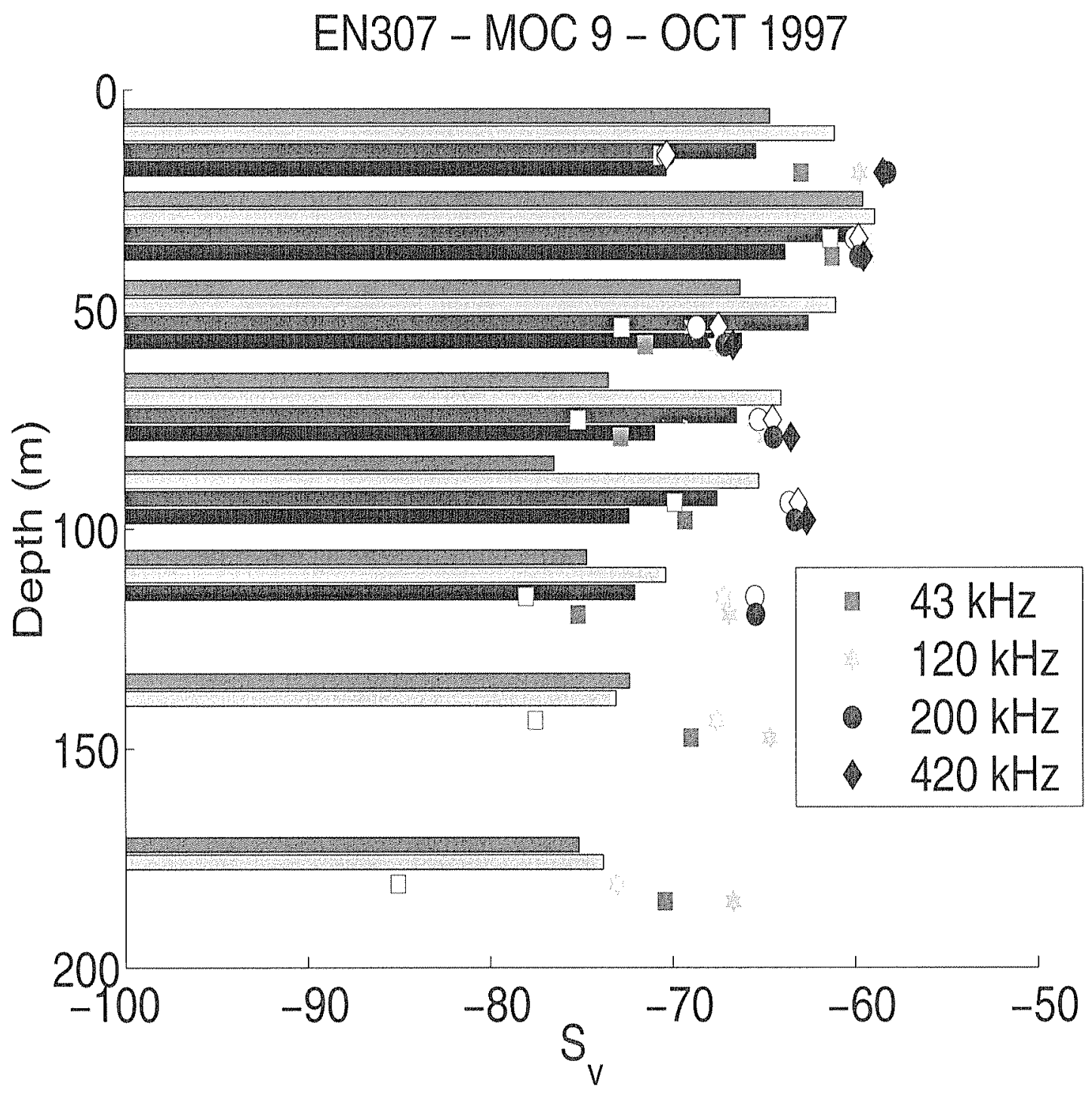

Figure 4-37: Measured values of volume scattering (filled in bars) for each frequency during MOCNESS \#9 compared with predictions from FP calculations both with (filled in symbols) and without (empty symbols). The predictions are plotted below the corresponding measured values. 
data then the volume scattering coefficient becomes a much more accurate measure of biomass in the ocean (compare Figures 4-38 and 4-37 with Figure 4-13). While there are still some discrepancies between the measured scattering and the biomass profiles, the overall trends and shape are correct. This shows that accounting for scattering contributions from physical processes are vital to properly interpret acoustic scattering data for information about biomass.

What these data clearly show though is the following, FP calculations that do not include scattering from physical sources underestimate the measured scattering. FP calculations that do include microstructure scattering tend to overestimate the measured amount of scattering and the errors from both these methods are similar. A comparison of Figures 4-37, 4-13,4-36, 4-35, and 4-38 demonstrates that the regions where there were large contributions to the scattering from physical sources are the same regions where the biomass and measured acoustic scattering differ. Acoustic scattering can not simply be converted to biomass estimates unless the sources of the scattering and their relative contributions are known.

\subsubsection{Inversion of Multiple Frequency Acoustic Data for Biological and Physical Parameters}

The spectral information from the BIOMAPER-II data from the first transect through the internal wave (Figure 4-3) was combined with predictions from theoretical scattering models for microstructure and euphausiids to provide information about these scattering processes. Various values of $\epsilon$ ranging from $10^{-10}$ to $10^{-4}$ were used with an average temperature and salinity profile for this region (from MOC \#9) to predict what the scattering spectrum for microstructure would be (Figure 4-39). A least squares fit of the measured spectra of the upper layer of the internal wave to these data provides an estimate of $\epsilon$ for this region. A prediction (from acoustic data) of $\epsilon=0.7 \times 10^{-6}$ was found, which agrees well with published values of $\epsilon$ in turbulent regions (Hebert et al., 1992; Seim et al., 1995). This estimate is also quite close to the value found with the Thorpe Length method for MOC \#9. For the upper 20 meters 


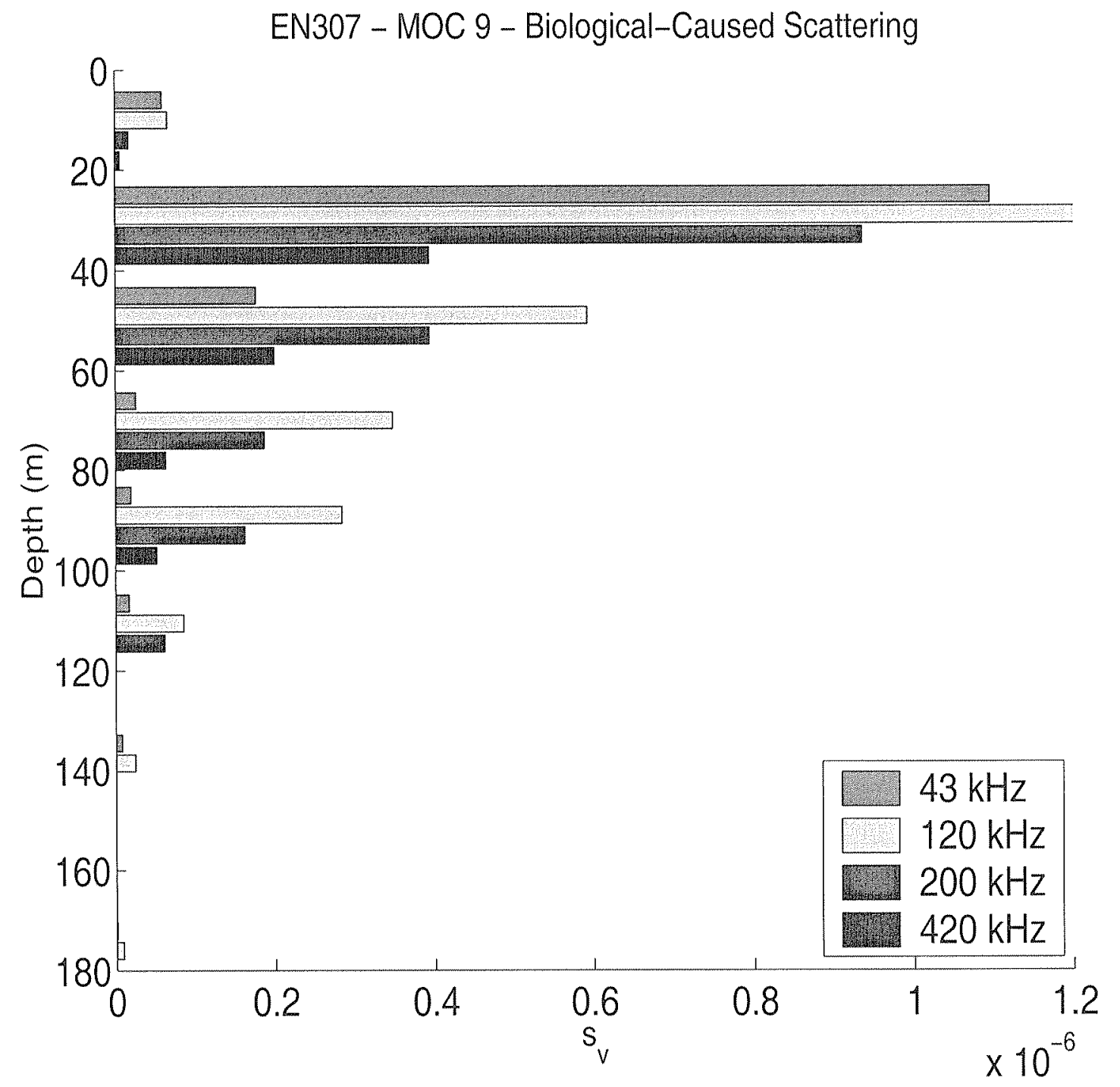

Figure 4-38: Predicted values of volume scattering coefficient from biological sources of scattering for each frequency during MOCNESS \#9. There is a strong agreement between these data where scattering contributions from physical sources have been removed and the depth profile of zooplankton biomass. 
of the water column (where the upper layer of the internal wave was observed), the mean value of $\epsilon$ was found to be $1.8 \times 10^{-6}$. Therefore, the inversion of acoustic data for the upper layer of the internal wave provides a parameterization for the dissipation rate of turbulent kinetic energy.

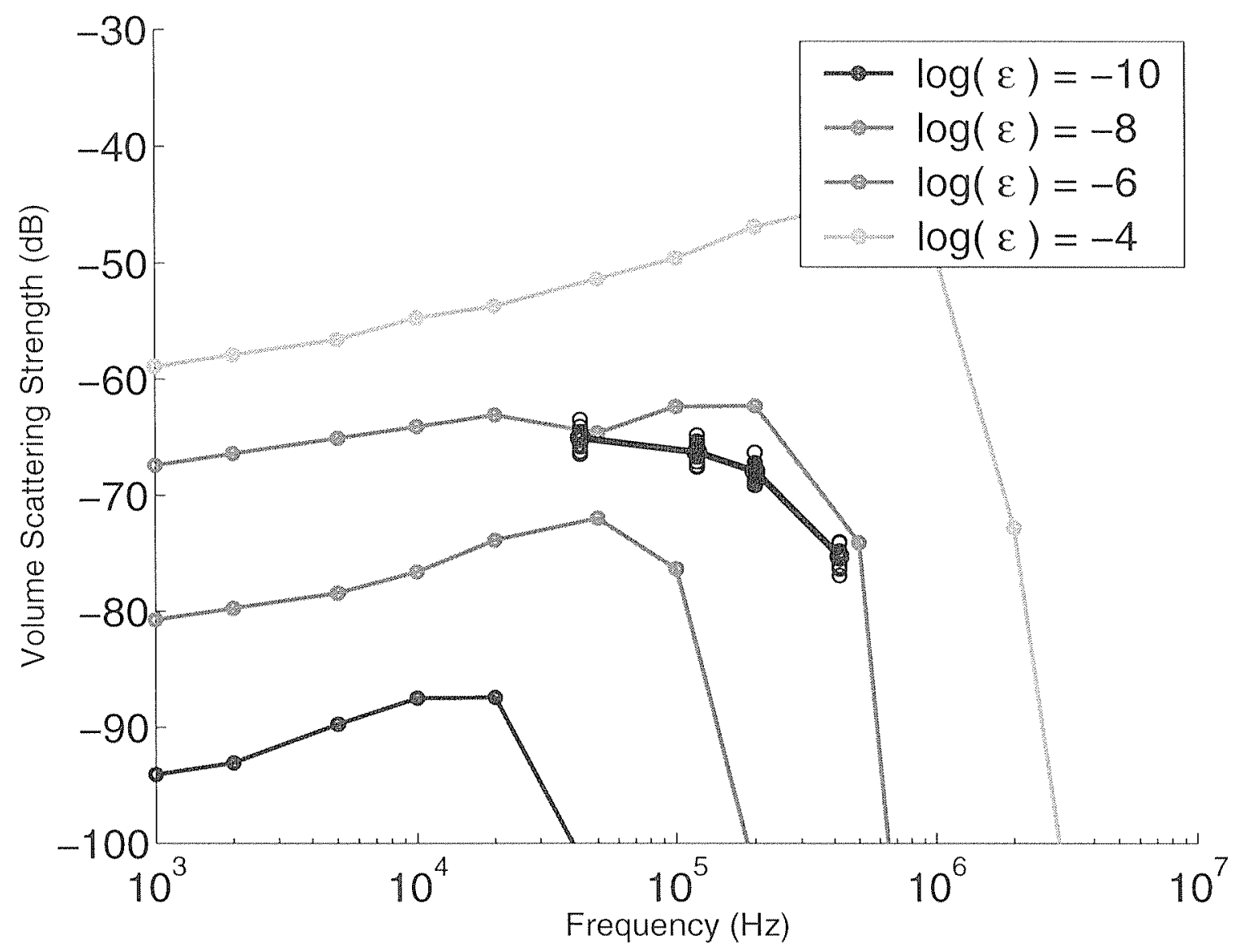

Figure 4-39: Observed scattering spectra for the upper layer of an internal wave (thick line) compared with theoretical scattering spectra predictions for a wide range of values of $\epsilon$. The least squares fit of the theoretical curves to the observed data provides an estimate of $\epsilon=0.7 \times 10^{-6}$ which is comparable to observations made by other scientists in similar high-energy regions, and also to the estimate of $\epsilon=1.8 \times 10^{-6}$ that was found for the upper 20 meters of the water column for MOC \#9.

A similar inversion was performed for the data for the lower layer of the internal wave (Figures $4-2$ and 4-3) which had a scattering spectra consistent with that of biological-caused scattering. The lower layer of the internal wave was observed from approximately 50 - 100 meters depth. Zooplankton scattering predictions from from MOC \#9 (Figure 4-35) show that the scattering from 60 - 80 meters depth (sampled 
by net \#5) was dominated by euphausiids. The scattering model used for a euphausiid (Figure 4-29a) was run for various sizes of euphausiids (ranging from lengths of $1 \mathrm{~mm}$ to $9 \mathrm{~cm}$ ) and a least squares fit of the "turning point" of the theoretical predictions with the scattering spectra data for the lower layer of the internal wave provides an estimate of the size of the euphausiids (Figure 4-40). This length estimate, found from acoustic data, was $1.55 \mathrm{~cm}$. The measured length of euphausiids from net \#5 from MOC \#9 was $1.51 \mathrm{~cm}$, so the acoustic predictions are quite accurate.

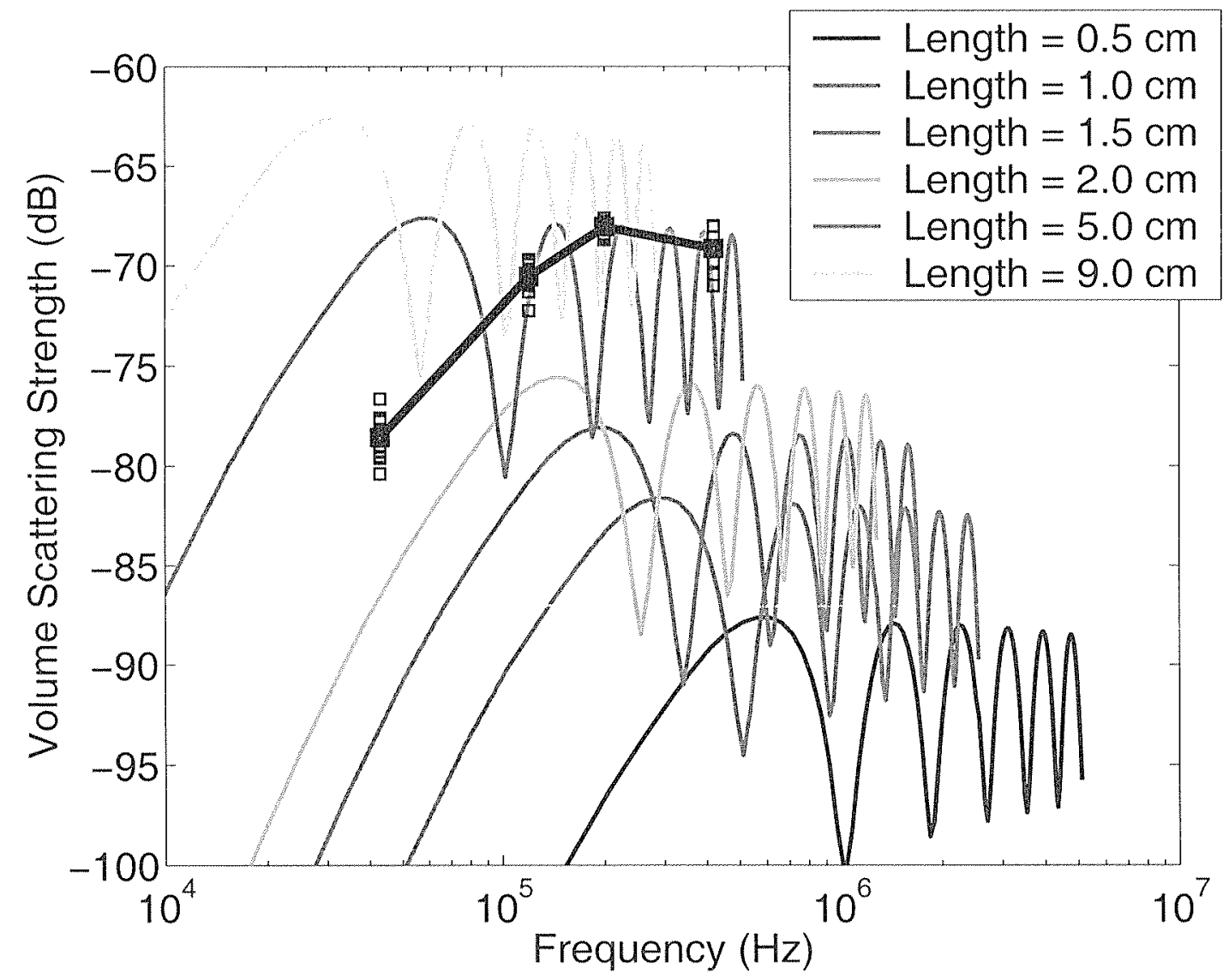

Figure 4-40: Observed scattering spectra for the lower layer of an internal wave (thick line) compared with theoretical scattering spectra predictions for a wide range of sizes of euphausiids. The least squares fit of the theoretical curves to the observed data provides an estimate of the length of the euphausiids of $1.55 \mathrm{~cm}$ which is comparable to measured values of length of $1.51 \mathrm{~cm}$ for the euphausiids caught in net $\# 5$ of MOC $\# 9$ (which sampled the same depth range that the lower layer of the internal wave was observed).

These two results show that multiple frequency acoustic data combined with the- 
oretical scattering models can provide information about both biological-based and physical-based scattering processes occurring in the water column. Using the slope of the scattering spectra to differentiate whether the scattering is biological or physical, appropriate scattering models may be fit to the observed scattering spectra to provide descriptions of turbulence $(\epsilon)$ or the size of certain zooplankton.

\subsection{Discussion}

\subsubsection{Distinguishing Between Different Sources of Scattering: Zooplank- ton and Microstructure}

It is still not possible to determine exactly what scattering process is occurring in the ocean from multiple frequency acoustic data. However, by combining the spectral information from an echo-sounding system with theoretical models of the scattering from biological and physical sources, scientists have a useful technique to discriminate between these scattering processes. It is important to mention though that groundtruthing is still required in order to properly interpret acoustic data. In addition to net tows to collect biological scatterers, if meaningful analysis of scattering from microstructure is desired then high resolution information about the hydrography of the water column is also needed.

Previous work has focused on the use of spectral information to distinguish between either different size classes of zooplankton (Holliday, 1977) or different taxonomic groups (Martin et al., 1996; Chu and Stanton, 1998; Martin-Traykovski, 1998). Fluidlike animals and elastic-shelled animals have "positive" scattering spectra (for the frequencies used by BIOMAPER-II), however gas-bearing animals have a somewhat flat spectra at these same frequencies. It has been proposed that these differences may be large enough that they could be used to discriminate between assemblages of the different animals. However this theory does not account for non-biological scattering processes contributing to the measured level of scattering. In many oceanic

environments, this is a valid assumption; however there exist numerous regions of the 
ocean where physical scattering processes are strong enough to provide detectable amounts of acoustic scattering (for example Haury et al. (1983); Sandstrom et al. (1989); Seim and Gregg (1994); Trevorrow and Teichrob (1994); Trevorrow (1998)). Many of these environments are rich in zooplankton and fish specifically because of these physical processes which can control numerous biological processes such as predator-prey interactions, encounter rates, and feeding behavior.

This study shows that different regions of the water column have acoustic scattering spectra that may change in both time and space. Initially it was thought that the different taxonomic groups of zooplankton (which can have quite different looking scattering spectra) were the cause. However, the net tow data were not conclusive in categorizing the different regions (determined by scattering spectra) as having different biological compositions. Scattering from physical processes was then proposed as contributing to the scattered field that was seen in the internal wave study.

Using the hydrographic data that was collected, levels of turbulence were derived from the acoustic data. These estimates are quite reasonable for this area of the ocean (Rehmann and Duda, 2000; Sandstrom et al., 1989). While acoustics may not the best method for determining the turbulence level in the water column, the advantages of this method in speed and spatial coverage merit further investigation.

\subsubsection{Contributions of Microstructure to Measured Scattering}

This work is the first time that scattering contributions from both biological and physical sources have been quantified in an ocean environment. Unfortunately, there was not the proper instrumentation on board to measure the dissipation rates of turbulent kinetic energy and temperature variance which are vital inputs into the theoretical microstructure scattering models. Therefore a way of estimating these inputs was used which is less than ideal. While this method provides reasonable estimates of $\epsilon$ and $\chi$, there are likely to be errors resulting from the inputs to the model. These errors in the model output predictions of scattering levels are easily seen. In some regions of the water column, $s_{v}$ values from microstructure alone 
are larger than the measured scattering from BIOMAPER-II. However, the errors associated with the microstructure-caused scattering are of the same order as the errors from the biological-caused scattering.

While the errors that we are discussing are relatively small (on the order of 5 $-10 \mathrm{~dB}$ ) given the range of scattering levels seen in the ocean (-50 to $-100 \mathrm{~dB})$, they become very large when converted from acoustic scattering levels to estimates of biomass. A $3 \mathrm{~dB}$ difference in scattering level is a factor of 2 when converted to biomass, therefore our predictions result in estimates of zooplankton abundance (one of the goals of this work) which are correct to an order of magnitude. In some cases this may be acceptable, but further work on reducing the error is still most definitely needed.

\subsubsection{Inversion of Acoustic Data for Estimates of $\epsilon$}

Given the aforementioned difficulties in obtaining accurate measurements of $\epsilon$ and $\chi$, acoustic data were inverted to find the value of $\epsilon$ in the water column by assuming that once the biological-caused scattering was accounted for, the remaining scattering was caused by microstructure. This method is not meant to be used as a means to measure $\epsilon$ indirectly, but was used to see if the scattering that we could not account for could be caused by a realistic level of turbulence in the water column. The results of this inversion of acoustic data in conjunction with the theoretical scattering model show that levels of turbulence that are common in the waters of the Gulf of Maine could cause levels of scattering which we have observed. This work was the first to use acoustical information to quantify levels of $\epsilon$ in an ocean environment, however this was merely an exercise to reassure us that our model predictions were reasonable.

The output of the scattering model for microstructure suggest that acoustics may be able to offer insight into the length scale of the mixing processes in the ocean. If the "leftover" scattering after biological processes have been accounted for is assumed to be from physical processes, then these values of $\sigma_{b s}$ or $s_{v}$ may be inverted for the levels of turbulence $(\epsilon)$ for each acoustic frequency and water column depth. It was found 
that the scattering strength varied with depth and frequency. Since the scattering from microstructure is a function of the length scale of the microstructure relative to the acoustic wavelength, it is possible that these variations with respect to frequency are the result of the mixing processes (and resultant microstructure) having varying length scales. Further work is needed to prove that this is indeed occurring, but the data are consistent with this theory.

This variability in the shape of the scattering spectra from microstructure complicates the process of determining the cause of the scattering. The model for microstructure-caused scattering has a peak at a frequency which is related to the length scale of the microstructure. Different length scales of microstructure in the ocean will shift the scattering spectra in either direction relative to acoustic frequency. Since the BIOMAPER-II system makes measurements at only five frequencies (and only four are used in this analysis), the slope of the spectra could be flat, positive, or negative depending on the relative size of the microstructure length scale. Therefore, unless the scale of the microstructure is known to some extent, interpretation of scattering spectral information based only on the acoustic measurements is a nebulous endeavor. If high-resolution CTD information is also collected, then the length scale of the microstructure can be found which would allow the scattering spectra to be modeled accurately and determine if the microstructure spectra would be positive, negative or flat in a particular environment.

In theory, the methods for estimating dissipation rates of turbulent kinetic energy could be used to conduct surveys of the entire water column (limited by the range of the acoustic sensors). Three dimensional maps of physical oceanographic parameters could be created similar to the spatial curtain plots that are frequently made for zooplankton biomass. Measurements of this nature would be extremely valuable in understanding physical oceanographic processes since acoustic surveys could be conducted more quickly than the high resolution CTD casts which are currently required. CTD measurements would still be required but there would be far fewer of them since the acoustic data would provide data between the casts. 
Additionally, these maps of $\epsilon$ may offer insight into the interactions between biological and physical processes. Many aspects of zooplankton behavior (such as swimming rates, predator-prey encounter rates, dispersion of larvae) are known to be influenced by the turbulence, mixing, and movement of their environment. So an understanding and ability to measure the rates of certain physical processes combined with information about zooplankton distributions may allow a greater understanding of these complex interactions. Conversely, there are also physical processes (such as gas transfer or solubility) that are influenced by biological processes (such as abundance

of phytoplankton and their predators or utilization of oxygen by zooplankton). If one is able to develop spatial maps of the various biological and physical parameters, then the study of their resulting interactions becomes much more feasible. While the methods outlined in this thesis are still preliminary, it is worthwhile to examine how these data may be used to study these interactions.

\subsubsection{Implications for Single and Multiple Frequency Acoustic Surveys}

The use of acoustic surveys to quantify and map distributions of zooplankton is becoming increasingly popular. However, interpretation of the acoustic data is never a straight-forward problem. Unless the zooplankton are identically the same in size, shape, species, and orientation; conversion from $s_{v}$ to biomass is not a simple task. Many scientists have been pleased by the success of using single-frequency echosounder systems to estimate levels of biomass (and in terms of labor, time, and spatial coverage; acoustics are the best device to use for this). Most surveys will collect "ground-truthing" data via video or net tow sampling in order to verify that the acoustic data are accurate; however few, if any, of these surveys are regularly using the hydrographic information to determine the structure of the water column and how it may contribute to the acoustic scattering.

Given that in some regions (such as the internal wave dominated area of this study) scattering from microstructure can be equivalent to that of biological sources, high resolution CTD measurements become as important to collect as net tows. Addition- 
ally, if one wishes to discriminate between different sources of scattering, then more than one frequency of acoustic echosounder must be used. Whether differences between two frequencies are used, or slope information from more than two frequencies, a single frequency echosounder by itself can not provide any information about the source of the acoustic scattering it is measuring.

Therefore, unless an oceanic region is dominated by a single type of scatterer (such as a single taxa), multiple frequency systems are needed to properly interpret acoustic scattering information. While the costs associated with these systems are much higher than a single frequency system, this is the price one must pay for accurate and believable measurements. It should be noted that a multiple frequency system by itself will not provide further insight into determining whether zooplankton or microstructure or other sources are scattering the sound. Understanding and proper use of theoretical scattering models, for different types of zooplankton and microstructure, and ground truthing measurements (net tows and hydrographic profiles) are an absolute requirement in order to estimate the distribution and abundance of marine animals from acoustic surveys.

Future surveys of zooplankton populations could use the results presented in this thesis to determine the relative amount of scattering that is from biological and physical sources using standard oceanographic equipment (CTD casts). While these techniques are not fully developed, they are mature enough to provide a substantial improvement in the interpretation of acoustic scattering data for biological information. Further work needs to be done in this area including laboratory measurements of scattering from temperature and salinity microstructure, as well as testing these methods in other areas of the ocean. However, a large increase in the understanding of the distribution of zooplankton is gained by using the methods outlined herein.

\subsubsection{The Inverse Problem}

The goal of conducting acoustic surveys of biological organisms is to increase our knowledge of the behavior, abundance, and distribution of the zooplankton. In order 
to take advantage of the benefits of acoustical methods, the inverse problem needs to be solved. However, this thesis has stated that the inverse problem can not be solved accurately unless the forward problem is well posed. A well posed forward problem implies that predicted levels of scattering agree with measured values of scattering which occurs now only occasionally.

There are several areas of research that need to be addressed to continue to improve the accuracy of these forward problem calculations. Most importantly is further analysis on the contributions of scattering from physical processes such as temperature and salinity microstructure and turbulence in the water column. This thesis has shown that accounting for the acoustic scattering contributions from these processes leads to an improvement in the use of acoustic backscattering data as a measure of zooplankton biomass. Further work needs to be done to determine the natural behavior and orientation of many types of zooplankton, discriminate between different types of zooplankton using spectral differences, and implement broadband, high frequency acoustic systems to collect spectral data in the geometric scattering region.

While these topics are necessary for improving the accuracy of forward problem calculations, if physical scattering processes are included, then the forward problem may be well posed enough to attempt to solve the inverse problem. The benefits of developing a solution to the inverse problem are enormous and would lead to a much better understanding of many biological and physical processes. It should be noted that there is likely to never be a single, correct solution to the inverse problem due to the complexity and variety of scattering processes that occur in the ocean, however a range of solutions and error estimates is quite possible.

The next step that needs to be made is to develop a methodology for using spectral information about the scattering to distinguish regions of the water column with different scattering processes. CTD and zooplankton net tow information will supplement this information, and will also be used to determine the contributions of the various scattering processes to the overall predicted level of acoustic backscatter. Once the relative contributions of each scattering process are known they can be 
combined with the measured data to determine the measured level of scattering for a region from each type of scattering process. With this information, the inverse problem may be broken down into a series of inverse problems for each type of scattering process. Solutions of the inverse problem for a single type of scatterer are more likely to be accurate, and by separating the overall inverse problem into a subset of inverse problems for each scatterer type (microstructure, euphausiids, salps, pteropods, etc.) there is a greater chance for accurate output.

There are still many difficulties with this approach. CTD and net tow stations are generally separated by considerable differences in both space and time. Additionally, zooplankton distributions will also change in space and time. Therefore, the ideal data set to begin this inverse problem analysis would be a simple transect through a region with CTD and net tow stations at the end points and the overall duration of the experiment to be less than 12 hours (to minimize the effects of vertical migration). The ability to conduct this experiment, already exists and, in fact, data like this have most likely been collected on one of the five BIOMAPER Gulf of Maine cruises.

Identification of a suitable study region should be based on the following criteria: the endpoint net tows should have similar zooplankton constituencies and the CTD profiles at the endpoints should be similar. These two conditions would provide the best possible basis for the inverse problem to be correctly solved for the acoustic data collected between the two endpoints. Various methodologies for actually solving the inverse problem should be tried (and not limited to the basic non-negative least squares analysis), the results from different methods may provide a suitable estimate of the accuracy of this technique.

The tools necessary for solving the inverse problem are already in existence, what is needed now is an understanding of the various scattering processes that are occurring in the water column and this thesis has provided one method for determining this information. Whether or not it allows the inverse problem to be solved remains to be seen. 


\title{
Chapter 5
}

\section{Conclusions}

\author{
O plunge your hands in the water \\ Plunge them up to the wrist; \\ Stare, stare in the basin \\ And wonder at what you've missed.
}

W.H. Auden

This thesis examines the components to the Inverse Problem aimed at obtaining information about biological animals from acoustic surveys. Scattering models for fluid-like and elastic-shelled zooplankton were analyzed with scattering data from individual animals to verify these models and obtain information about the animal's size or orientation from single insonifications of a broadband signal. Next, a comparison between the scattering model predictions for a gas-bearing animal and in situ echo strength measurements was made. Finally, zooplankton and turbulent microstructure scattering models were used to interpret multiple frequency acoustic data from an acoustic survey of an internal wave.

The work presented in these chapters advances the ability of scientists to use acoustical scattering data to predict distributions of zooplankton. The importance of this work is stated quite simply in the final paragraph of a recent paper (Seim, 1999)

Multifrequency acoustic systems may be capable of distinguishing biology and microstructure in backscatter images based on spectral signatures. Validation of this ability will require accurate, simultaneous measurement 
of $\epsilon, \chi$, and $\sigma$, some confirmation of the viscous-convective subrange of salt, and zooplankton concentration measurements. The sampling requirements make this a daunting challenge, but the insight to be gained certainly will make the effort worthwhile.

This thesis has begun to answer this challenge and the results contained herein show that these methods have great promise for both differentiating between biological and physical sources of scattering and quantifying their respective contributions.

\subsection{Zooplankton Scattering Models}

Chapters Two and Three of this thesis explore the validity and variability of zooplankton scattering models for three types of animals. Fluid-like and elastic-shelled scattering models (Chapter Two) are used to provide information about either the size or the orientation of an individual zooplankter by examining the time and amplitude differences between multiple scatterings from the animal. For gas-bearing animals, the scattering model is validated by the collection of in situ echo measurements at multiple frequencies (Chapter Three). These data allow estimates to be made of the target strength of the animal in its natural environment. Using this information, echo integration data from a scientific echo-sounder aboard the same ship is used to estimate the numerical density of the siphonophores in the waters of Cape Cod Bay. In order to accurately use these zooplankton models in the Forward Problem calculations (Chapter Four), a basic understanding of the factors that will affect zooplankton scattering (animal orientation, size, behavior, and location in the water column) must exist.

\subsubsection{Effect of Animal Behavior}

Surprisingly little is known about the in situ orientation of most zooplankton species. There are several studies on the behavior and resultant orientation of euphausiids (Sameoto, 1980; McGehee et al., 1998), however there are few published studies about 
the typical orientation of other zooplankton (Benfield et al., 2000). There is also little known about the behavior of many of these animals and how it will affect, their orientation in the water column. Given that many studies (and theoretical models) have shown that a zooplankter's orientation may have a strong effect on the backscattered sound energy, the paucity of zooplankton orientation literature is a major hindrance in applying these models.

Innovative video techniques (such as the Video Plankton Recorder) and mesocosm experiments can provide information about the normal orientation of various zooplankton species in the water column, and how this orientation may vary during the course of vertical migration, feeding, or predator avoidance behaviors. Further work on refining the zooplankton scattering models may not be warranted until the issues of animal orientation and material properties (Chu et al., 2000a,b) are clarified. Given that a $3 \mathrm{~dB}$ change in Target Strength can occur between vertical and horizontal orientations, it is not clear whether more accurate scattering models are needed; or if, instead, the existing scattering models simply need better input data.

\subsubsection{In Situ Verification of Models}

Since many aspects of a zooplankter may change with the animal's vertical position in the water column (the resultant pressure effects will likely change the animal's size (particularly for gas-bearing animals) or the material properties $(g$ and $h)$ ), in situ verification of the Target Strength of animals is needed. For many smaller animals, it is near-impossible to measure the scattering from a single animal, especially in situ, but it is possible for measurements to be made on mono-specific assemblages (with animals captured for later taxonomic and size analysis). This is often done in field surveys, however it is very difficult to get a sample where only one taxonomic type is present. Again, mesocosm experiments may be an ideal situation to raise a single type of animal and perform acoustic scattering experiments on them. While not a true in situ experiment, these data would be vital to ensuring that the acoustic scattering models used are accurate. 
For gas-bearing organisms (such as the siphonophore) that migrate vertically hundreds of meters a day, a better understanding of the animal's regulation of the volume of the gas inclusion is needed. Since the acoustic resonance frequency is dependent on the bubble's size, changes in the volume of the gas inclusion will shift the scattering spectra relative to acoustic frequency. If the scattering spectra change with the animal's depth in the water column, then interpretation of acoustic survey data of these animals will be more complex. Significant errors can occur in biomass estimates of these animals if a static gas bubble size is used in the model and this assumption is not true.

\subsection{Accuracy of Forward Problem Calculations}

Previous studies applying zooplankton scattering models and field collected data to estimate the amount of backscattered sound energy in the ocean have been relatively successful (Wiebe et al., 1996). It is not realistic to expect these Forward Problem calculations to be completely accurate, there is too much variability in the ocean environment and the biological populations for the estimates to exactly equal the measurements. However, it is important to be able to explain why there are errors and what causes them. Initially in this work, the data collected were input into FP calculations using the scattering models used by Wiebe et al. (1996). The difference between measured and predicted scattering strength was of the order of 1 to $10 \mathrm{~dB}$, with the predictions both over- and under-estimating the measured amount of volume scattering strength. There were more underestimates than overestimates predicted by the Forward Problem which suggests that other non-modeled scattering processes may be occurring in the ocean.

These initial calculations did not incorporate any information about animal behavior or other scattering processes, so it is not surprising that there were errors in both directions (over and under estimates). The next step was to include information about animal behavior (specifically fluid-like zooplankton orientation). Using the conclusions from Chapter Two, the scattering model for some fluid-like zooplank- 
ton was modified to reflect scattering from animals at near-broadside orientation. Length-to-width ratios were also included to better reflect the actual shape of various types of animals. The result of this was to increase the scattering from these animals (although other animals such as salps and siphonophores contributed much to the overall scattering). This would, in turn, increase the overall level of predicted scattering (which would move the data values in the FP calculation figures towards the lower right corner). Since the changes in the scattering models for the zooplankton predicted an increase in the scattering from zooplankton (when it was hypothesized that the models were already overestimating the scattering), it is evident that further examination into the material properties of zooplankton is a necessary step in order to increase the accuracy of the zooplankton scattering models.

Finally, if the scattering from temperature and salinity microstructure is included in these calculations, then many of the underestimates of the measured scattering become overestimates due to the large amount of scattering predicted by the microstructure model. This is not surprising given that our estimates of $\epsilon$ and $\chi$ (which are inputs into the scattering model) are probably too large. This is most likely the result of the Thorpe Length method for estimating these parameters and if actual measurements of $\epsilon$ and $\chi$ were made, then the scattering contributions from microstructure may be reduced.

While the interpretation of acoustic scattering data for information about the biology present in the water column is not straightforward, there exists a wealth of theoretical and experimental evidence that show the great promise of this technique. Knowledge of the scattering processes occurring in the water column along with measurements of the relevant parameters offer the possibility of highly accurate estimates of the distribution and abundance of zooplankton.

By determining the relative contributions of the scattering processes to the total scattering in the water column, scattering from biological and physical sources can be determined. If these relative proportions are then applied to field collected acoustic scattering data, a depth profile of biologically caused scattering is found. These 
profiles agree very well with profiles of zooplankton biomass calculated from net tows. Our data show that if scattering contributions from physical sources are not determined, then interpretation of measured acoustic backscattering data from the water column will not lead to accurate estimates of biomass.

\subsection{Contributions of the Thesis}

- Obtained accurate estimates of the size of a fluid-like or elastic-shelled zooplankton from Pulse Compression processing of broadband echoes from an individual animal. Simple two ray scattering models accurately described the scattering processes occurring in fluid-like and elastic-shelled animals. Using a high-resolution $\left(1^{\circ}\right)$ data set, the effect of animal orientation on the scattered sound was found for these zooplankton.

- Developed an ROV-mounted multiple frequency acoustic array combined with a video camera system to record in situ backscattered echo measurements from siphonophores, a gelatinous zooplankter.

- Estimated the in situ Target Strength of siphonophores from in situ scattering measurements, combined these data with acoustic information from a downlooking echosounder to estimate the numerical density of the animals.

- Developed a method to use narrow band, multiple frequency acoustic scattering data to characterize regions of the water column based upon the "slope" of the scattering spectra. Regions with different slopes often had different scattering processes occurring. Showed that regions with identical scattering strengths at one acoustic frequency often have different causes of scattering.

- Inverted acoustic backscattering data for predictions of the dissipation rate of turbulent kinetic energy $(\epsilon)$ which agree with measured values from similar areas.

- Revised zooplankton scattering models used in Forward Problem calculations to include information about animal orientation and body shape. 
- Applied a theoretical model for scattering from microstructure to field-collected data from an internal wave in the Gulf of Maine. Results were combined with those from biological scattering predictions to fully characterize the scattering processes in the water column.

- Explained that differences in profiles of biomass and acoustic scattering are due to the presence of scattering from temperature and salinity microstructure. 


\section{Appendix A}

\section{Analysis of Biological and Physical Sources of Scattering from a Nearby Region}

\section{A.1 Acoustic, Environmental, and Net Tow Data for a Nearby Region}

The procedures used in this section are identical to those discussed in Chapter Four, therefore only differences in the data will be discussed. This set of data (MOCNESS \#7, CTD \#8, and associated acoustic records) was collected in the same geographical region (Figure 4-5), but two days earlier (14 October 1997).

\section{A.1.1 Acoustic Scattering Data}

The acoustic scattering from the water column clearly shows a distinct two layer pattern, similar to that of the internal wave transects. In this case, the upper layer shows stronger scattering at the lower acoustic frequencies, while the lower scattering layer is thicker and scatters more sound at the higher acoustic frequencies (Figure A-1). There are many strong scatterers seen on the lower acoustic frequencies at depths of $150-200$ meters. 


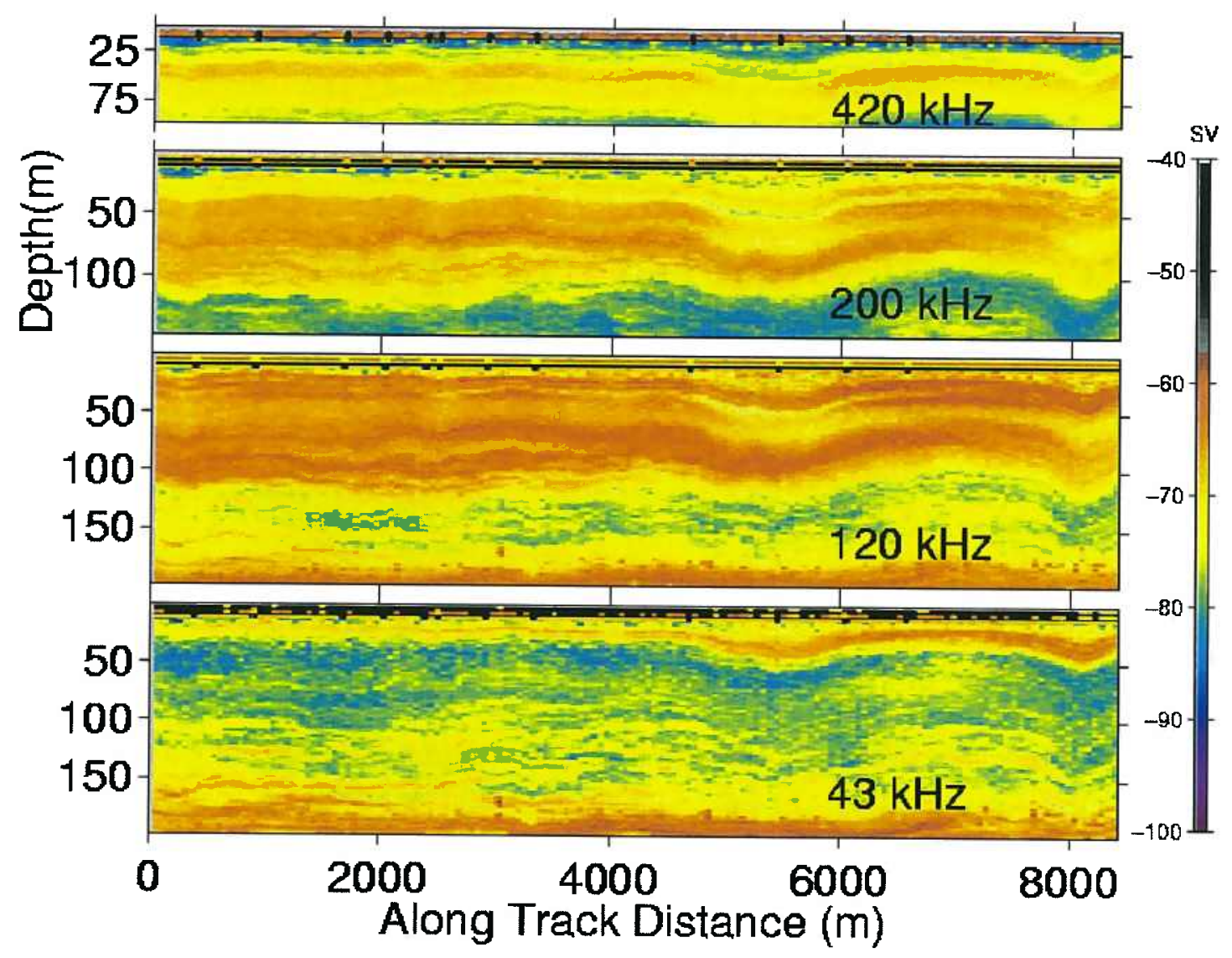

Figure A-1: Echogram of the volume scattering strength for the four frequencies $(43,120,200$, and $420 \mathrm{kHz}$ ) of BIOMAPER-II collected simultaneously during MOCNESS \#7. There is a strong scattering layer near the surface which shows up on all four frequencies (but is strongest at the lower frequencies), and a lower layer of scattering from approximately $100-150$ meters. 
In order to compare the acoustic regions with the MOCNESS information, the transect of the net can be overlaid on the acoustic plot to show which nets sampled which regions. The $120 \mathrm{kHz}$ plot shows strong scattering from nets \#5 - 8 (Figure A-2). The upper scattering layer is sampled by net \#8 (and possibly \#7 as well), while the lower scattering layer is sampled by nets \#5 and \#6.

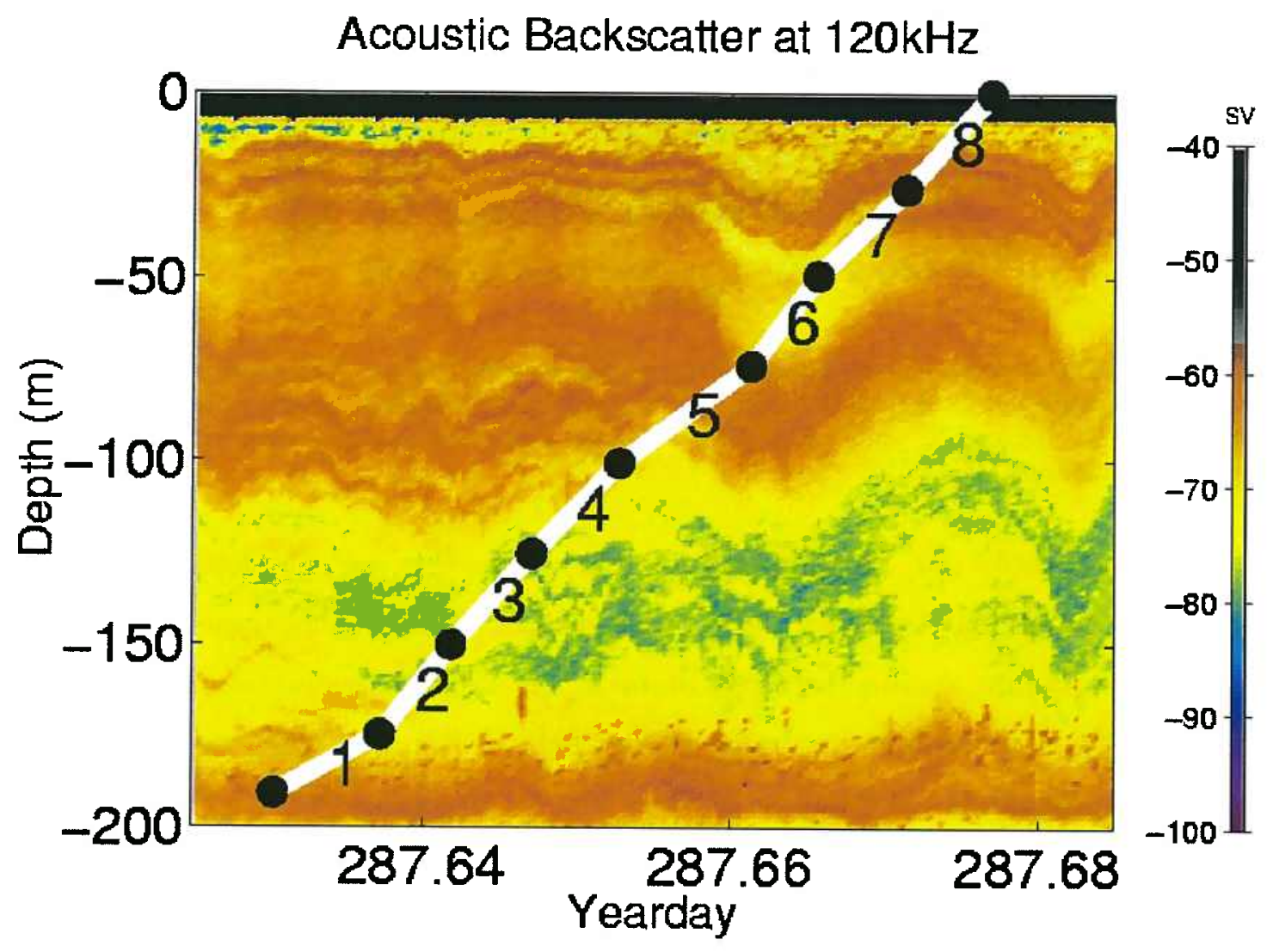

Figure A-2: The $120 \mathrm{kHz}$ volume scattering strength for MOCNESS \#7. The lower scattering layer is sampled by MOCNESS nets \# $5-7$.

The information from the multiple frequency scattering data shows that the upper and lower scattering layer have different spectral characteristics. The first-order "slope" of the scattering spectrum (using the 43,120 , and $200 \mathrm{kHz}$ transducers only) shows that a region of positive slope (consistent with scattering from fluid-like scatterers) occurs during nets \# 5 - 6 (with some overlap in net \# 7) (Figure A-3). Negative slope regions occur at the depths sampled by nets \# 3,4 and 8 . 


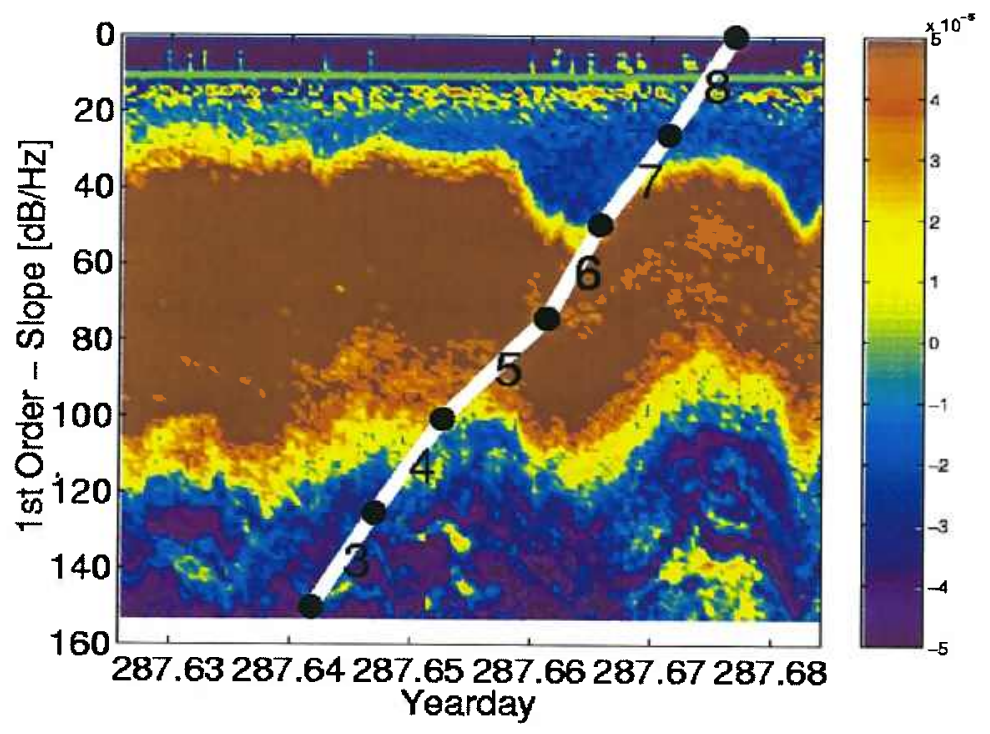

Figure A-3: Three frequency calculation of scattering spectral "slope" from the BIOMAPER-II acoustic data collected during MOCNESS \#7. A strong wave like feature with a positive slope is sampled by nets $\# 5$ and 6 , while nets $\# 3,4,7$, and 8 appear to sample regions containing both positive and negative slopes.

If four frequencies $(43,120,200$, and $420 \mathrm{kHz})$ are used to find the slope parameter, a similar picture is found (Figure A-4). These data are limited to the upper 100 meters of the water column, due to the range limits of the $420 \mathrm{kHz}$ transducer. These data show positive slope regions occurring during nets \#6 and 7, with negative slope regions for most of nets \# 5 and 8.

Second-order "slope" calculations (curvature and concavity) also indicate similar regions in the water column. The three frequency calculation of curvature indicates that nets \# 4 - 6 (and partially 7 ) are in a different region than nets \# 3 and 8 (Figure A-5). However the concavity calculations shows little variation in the water column. If four frequencies are used to find the curvature and concavity of the scattering spectrum, then these data clearly show that nets $\# 5$ and 6 occur in a different region than net \#8, with net \# 7 overlapping both positive and negative curvature and concavity values (Figure A-6). 


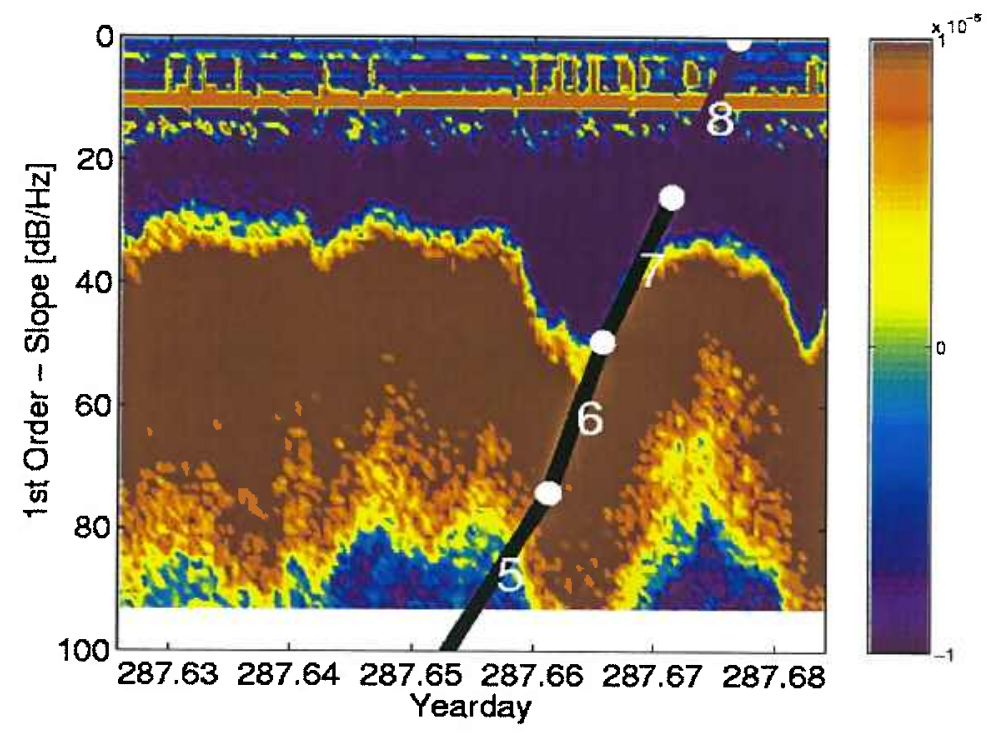

Figure A-4: Four frequency calculation of scattering spectral "slope" from the BIOMAPER-II acoustic data collected during MOCNESS \#7. A strong wave like feature with a positive slope is sampled by nets \# and part of net \#5, while nets \#7 and 8 appear to sample regions containing both positive and negative slopes.
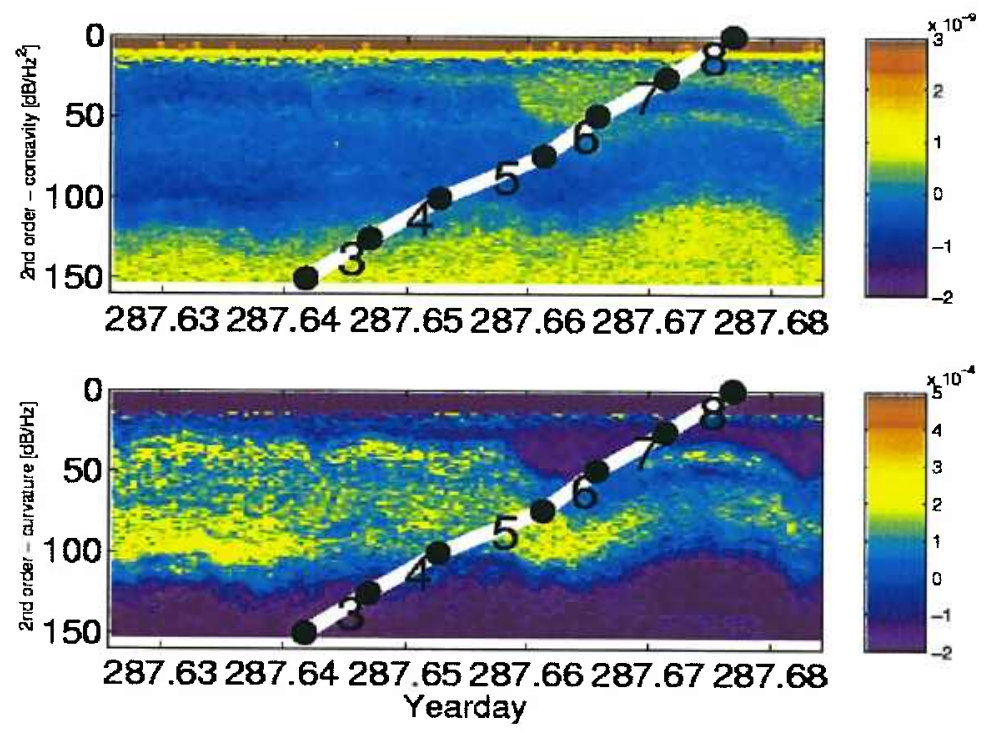

Figure A-5: Second order slope calculation from three frequencies of BIOMAPER-II during MOCNESS \#7. The same wave structure is evident in these plots as is seen in the first order slope calculation. 

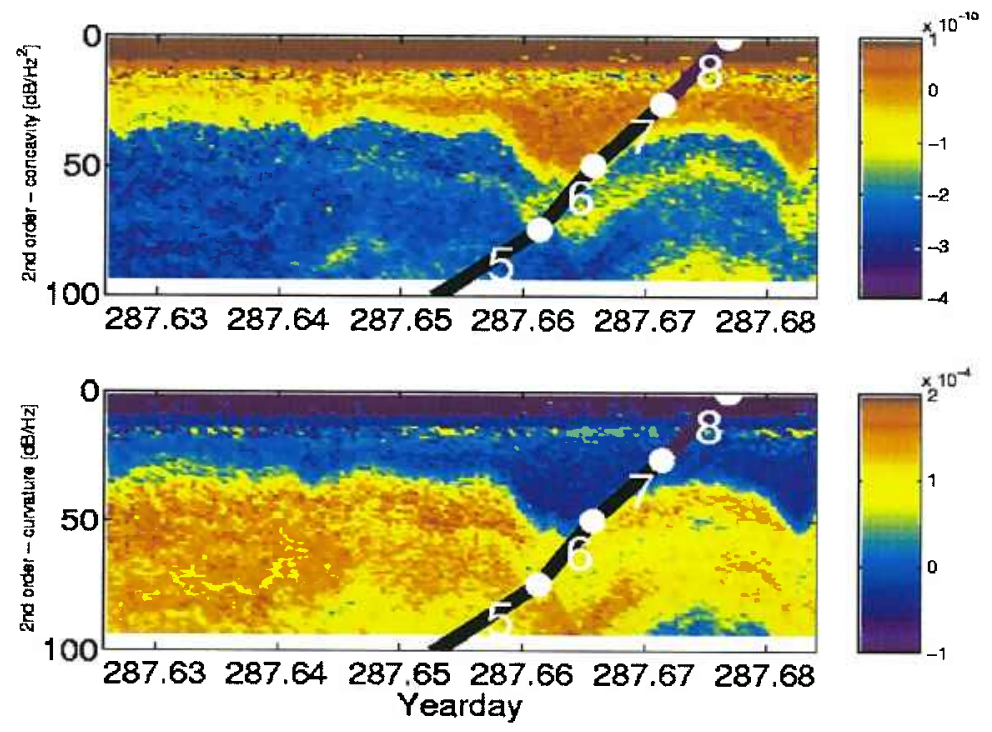

Figure A-6: Second order slope calculation from four frequencies of BIOMAPER-II during MOCNESS \#7. The same wave structure is evident in these plots as was seen in the first order slope calculation. 


\section{A.1.2 MOCNESS \#7}

The MOCNESS was lowered to 190 meters depth and was brought to the surface with nets closed and opened at $175,150,125,100,7550,25$, and 0 meters. The biomass profile versus depth peaked in the near-bottom layers, but the surface layer was also quite high in biomass (Figure A-7). The minimal amount of biomass found was in nets \# 5- 7 which sampled 25 - 100 meters depth, which is the same region that the lower acoustic scattering layer occurred. The samples were dominated by copepods with larger copepods being more frequent in the deepest nets (Figure A-8). Euphausiids were common below 100 meters depth and were particularly abundant in net \# 1 (the deepest net). In the surface nets (\#7 and 8), amphipods and chaetognaths were also found. There were very few gelatinous zooplankton found in these net samples which is a completely different taxonomic composition than what was found two days later in MOCNESS \#9.

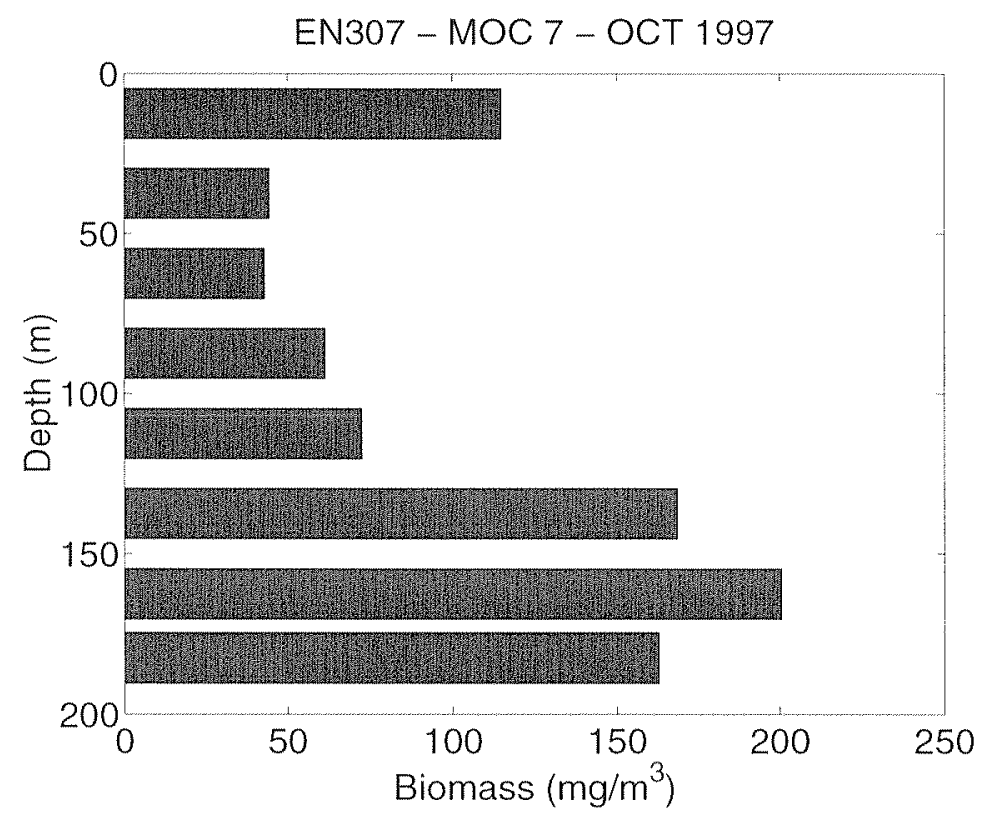

Figure A-7: Biomass estimates from MOCNESS \#7. A large amount of biomass was found in the deepest three nets, as well as in the surface layer from $0-20$ meters.

As occurred in MOCNESS \#9, copepods were found in all nets with large copepods (length greater than $2.5 \mathrm{~mm}$ ) more prevalent in the deepest three nets. Abundance 


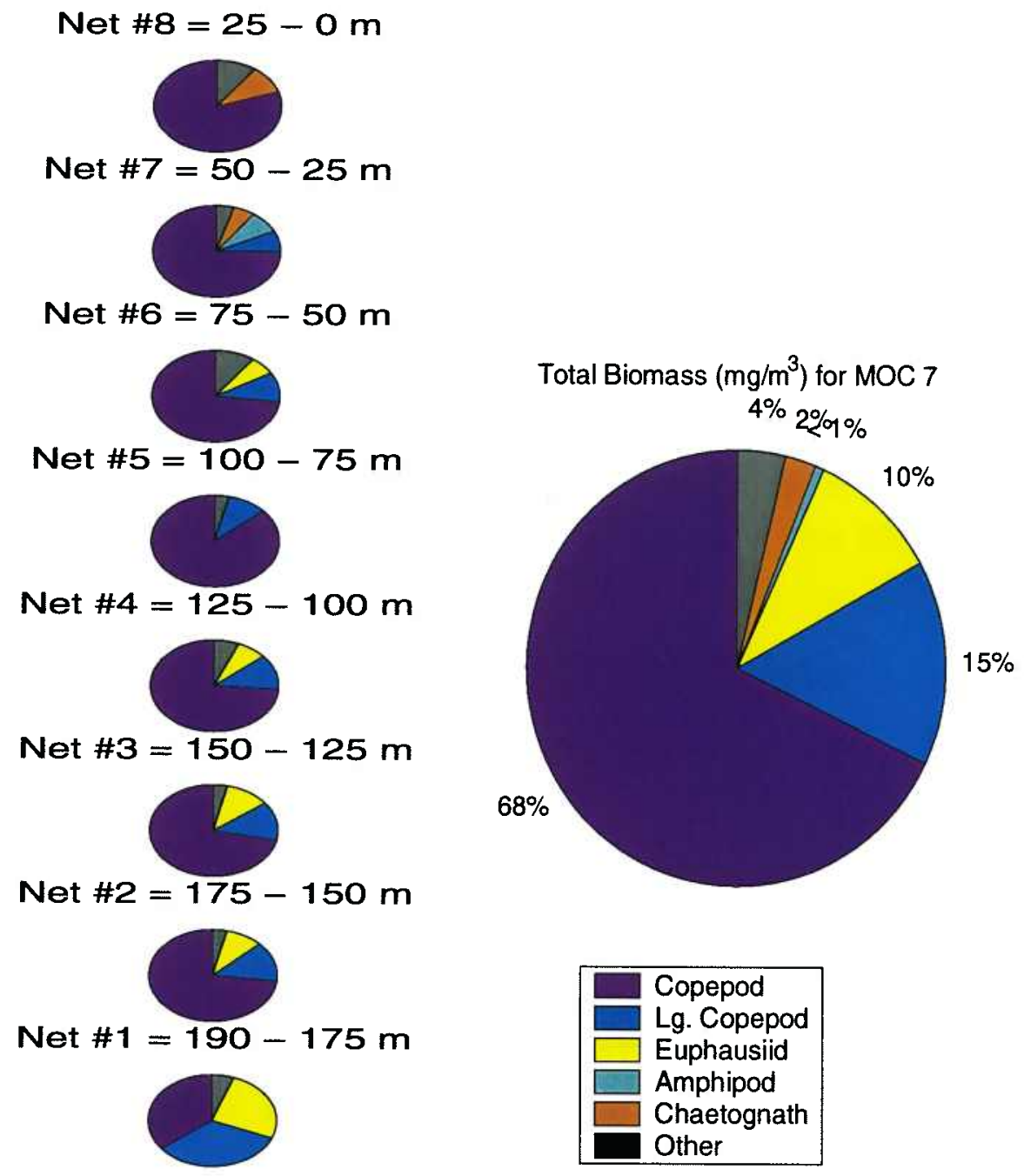

Figure A-8: Taxonomic composition of the biomass found in MOCNESS \#7. Copepods dominated much of the water column, however euphausiids contributed to the biomass in the lower three nets, and amphipods and chaetognaths were found in the surface layer. 
data show that copepods were numerically dominant in all nets (Figure A-9), while euphausiids were somewhat numerous in the lower nets. The presence of euphausiids (and their relatively high biomass per individual) explain the differences between the abundance distribution with depth (Figure A-10) and the biomass distribution (Figure A-7)

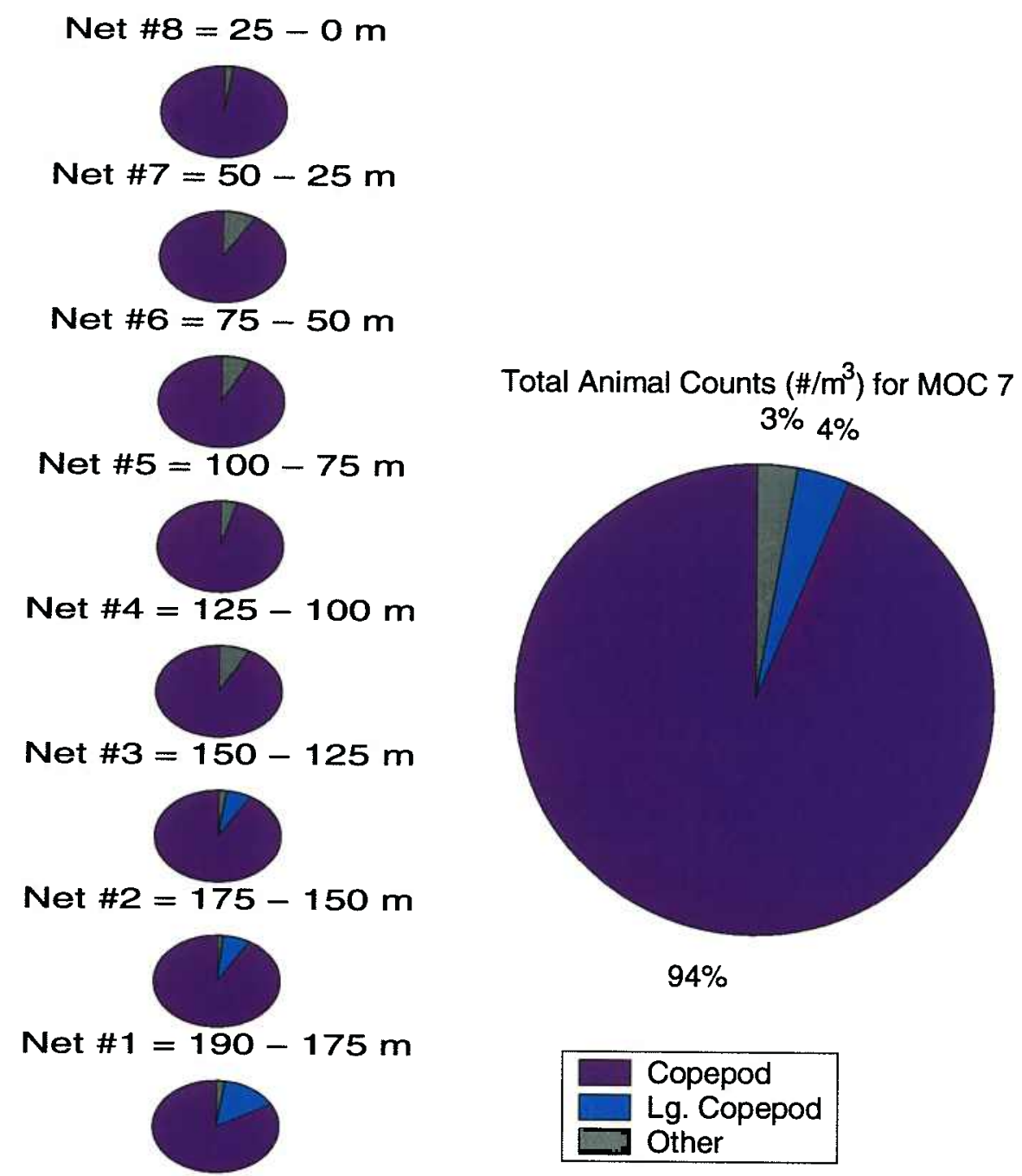

Figure A-9: Taxonomic composition of the numerical abundance data for MOCNESS \#7 for each net and overall. Copepods were by far the most abundant animal sampled, however due to their small size their contributions to biomass are not as great as their numerical proportion of the abundance. 


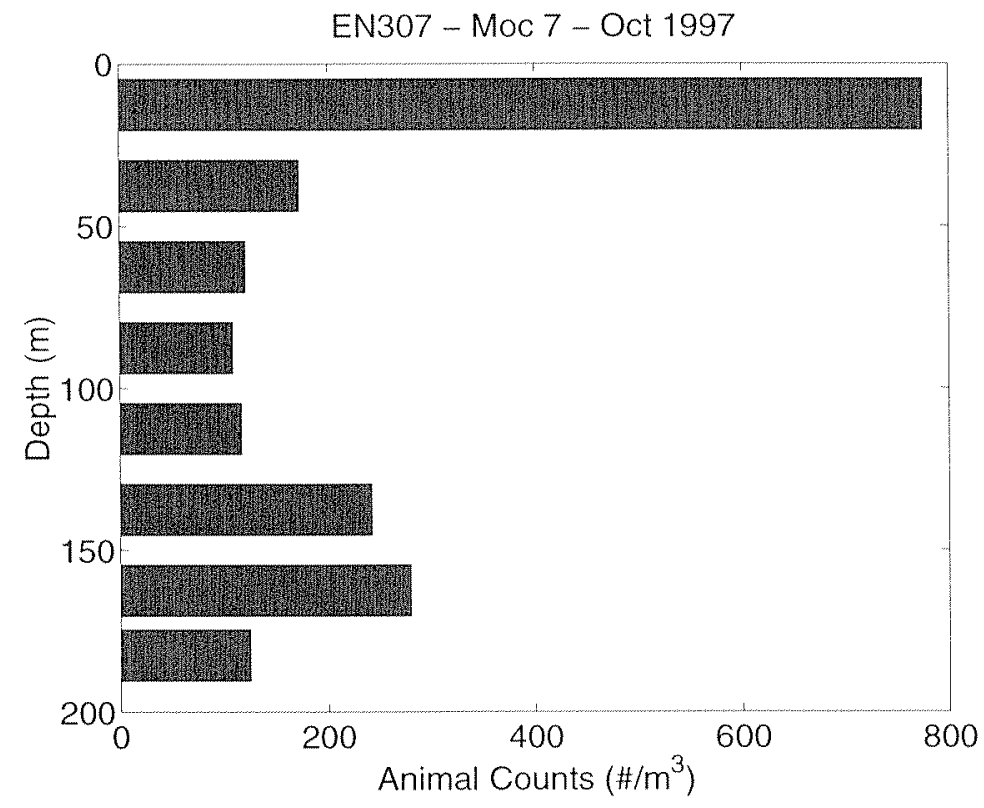

Figure A-10: Numerical abundance (animals $/ \mathrm{m}^{3}$ ) for each net from MOCNESS \#7. While more animals were found in the surface layer, their smaller average size results in the surface peak in biomass being smaller than the abundance peak relative to the rest of the water column. For the same reason, the lower water column (with more larger animals such as euphausiids) has a smaller abundance but a larger amount of biomass. 


\section{A.1.3 Water Column Hydrography}

The hydrography data from MOCNESS \# 7 show a complex water column with steep temperature, salinity, and density gradients in the top 30 meters of the water column. Immediately beneath this stratified region is a well-mixed region that goes to a depth of approximately 70 meters. The steep gradients continue for another 10-30 meters, and then another well-mixed region occurs at 90 - 110 meters. From 110 meters depth to the bottom of the sampled region (190 meters) there are small gradients of temperature, salinity, and density. (Figure A-11).

A comparison with the data collected by CTD cast \#8 shows how different the water column can become in a few hours. Although there is a large spatial distance between MOCNESS \#7 and CTD cast \#8 they are compared here since they were collected within hours of each other and are both located in the eastern edge of Wilkinson Basin. The hydrographic profile (Figure A-12) shows a completely different looking water column with a well-mixed surface layer followed by steep and then gradual gradients in temperature, salinity, and density. This profile shows many overturning density layers from 30 - 60 meters which suggest some type of mixing event is occurring in this region. The MOCNESS CTD record shows this same region as being well-mixed, so it's possible that we are sampling this region before and after a mixing process is occurring.

\section{A.1.4 Forward Problem Calculations}

The Forward Problem calculations for MOCNESS \#7 have both over- and underestimates for the predicted scattering models (Figure A-13). Overall, most of the predictions are underestimates of the measured scattering levels, with the exception of the $120 \mathrm{kHz}$ data for the bottom three nets and the $200 \mathrm{kHz}$ data for the nearbottom nets. Unlike the FP calculations for MOCNESS \#9, there do not seem to be any patterns or relationships between acoustic frequency or net number and whether the predictions are over- or under-estimates of the measured scattering levels. If the scattering contributions are examined on a taxonomic basis, the dominant scatterers 


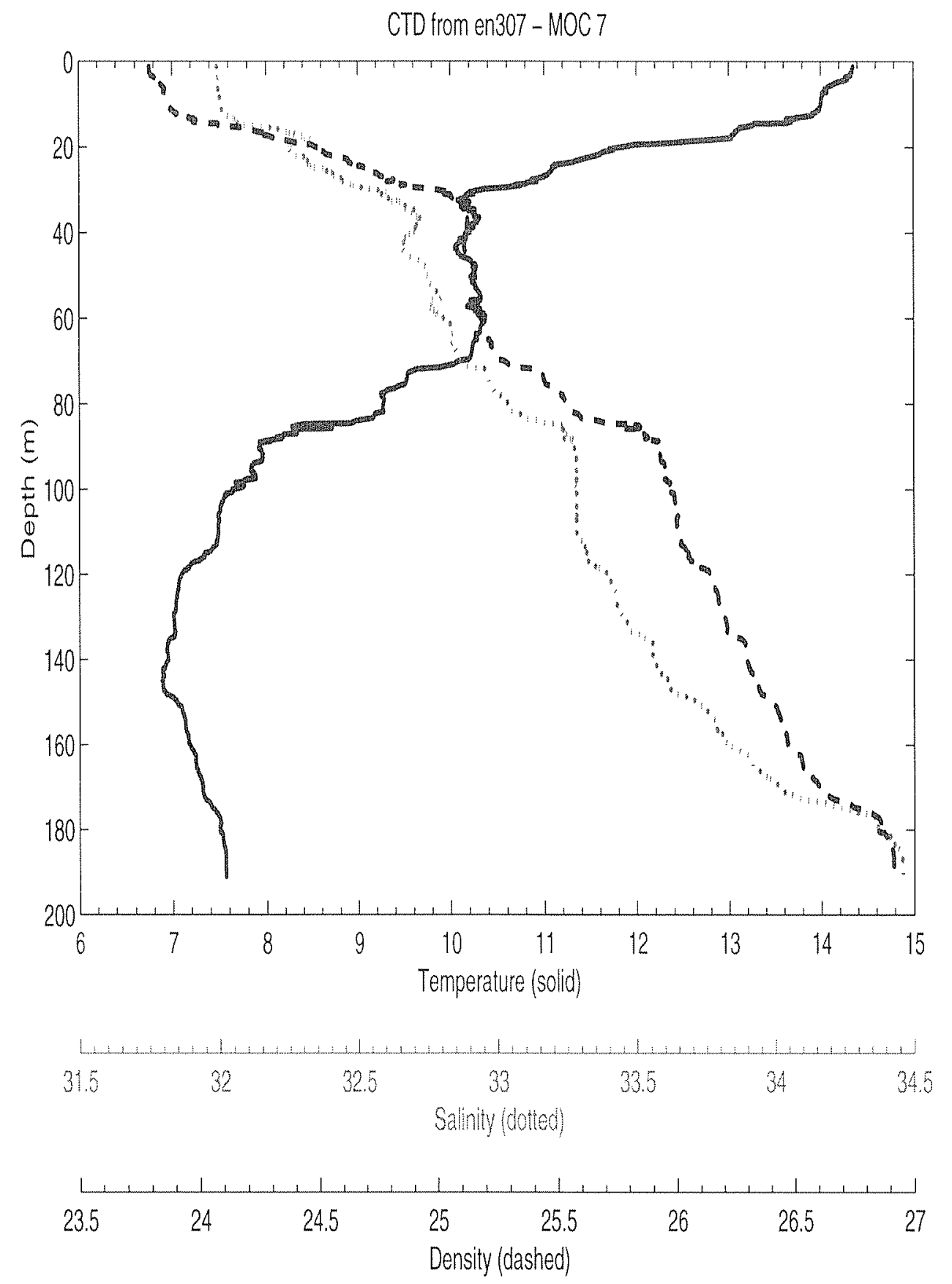

Figure A-11: Temperature, salinity, and density profiles for the water column sampled by the ESS system on board MOCNESS tow \#7. The upper 20 meters appear to be well stratified, but there is a well-mixed region from $30-80$ meters. This region corresponds to where the lower scattering layer was seen in the acoustic data during this tow so it is possible that this region has been mixed by an internal wave. There are also smaller mixed regions throughout the water column, however this profile is more stable than that for MOCNESS \#9 

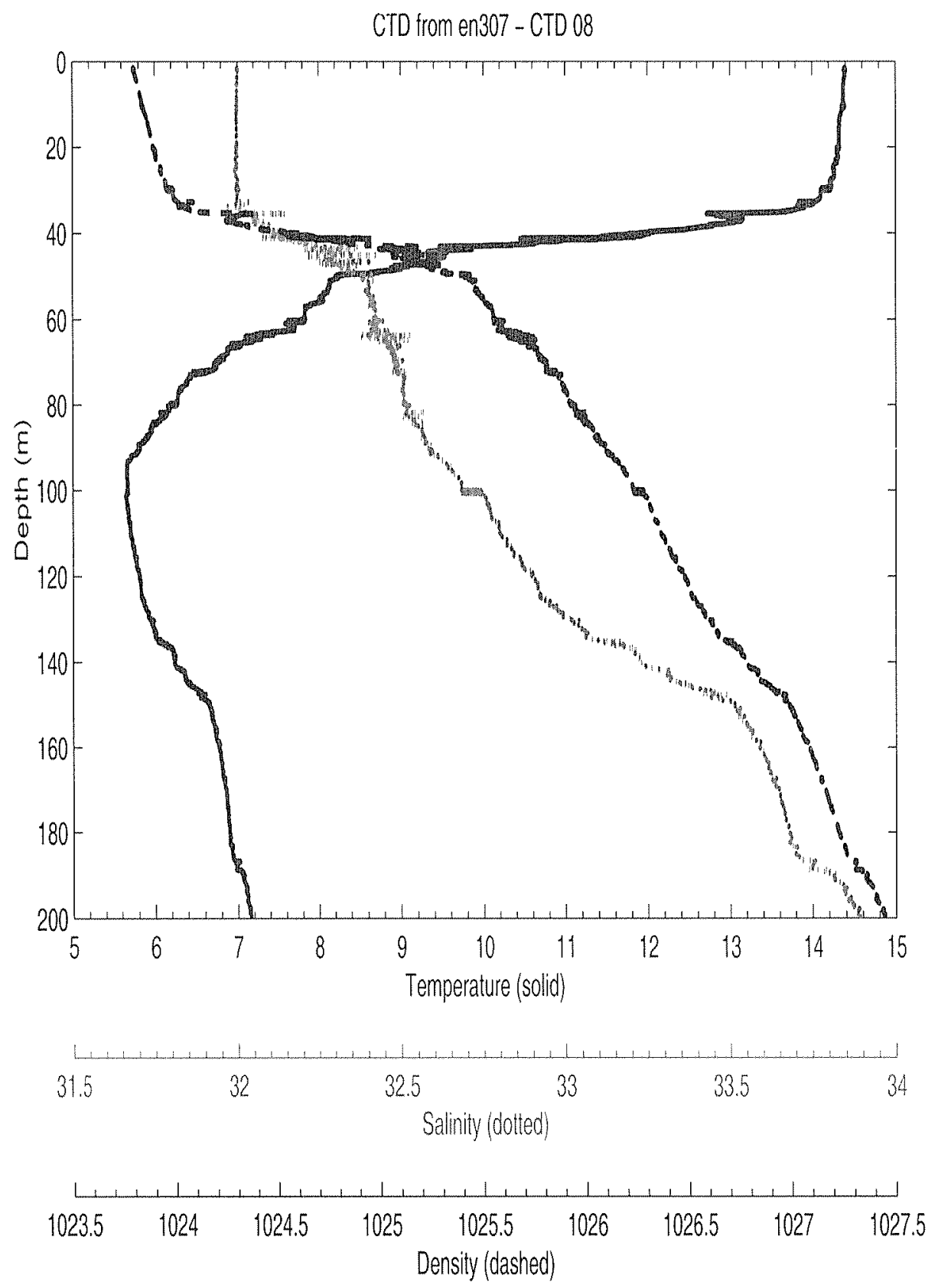

Figure A-12: Temperature, salinity, and density profiles for the water column sampled by CTD cast \#8 conducted shortly before MOCNESS \#7, however several kilometers away. The water column appears stratified with a well-mixed region from the surface to 30 or 40 meters depth. Below this mixed layer there are small regions of density instabilities. A comparison of this profile with that for MOCNESS \#7 shows how much the hydrography of this region can vary in space and time. 
are euphausiids, amphipods, chaetognaths, pteropods, and parts of siphonophores (nectophores and pneumatophores) (Figure A-14). The other animals (particularly the abundant copepods) contribute little to the overall predicted scattering, especially at the lower frequencies.

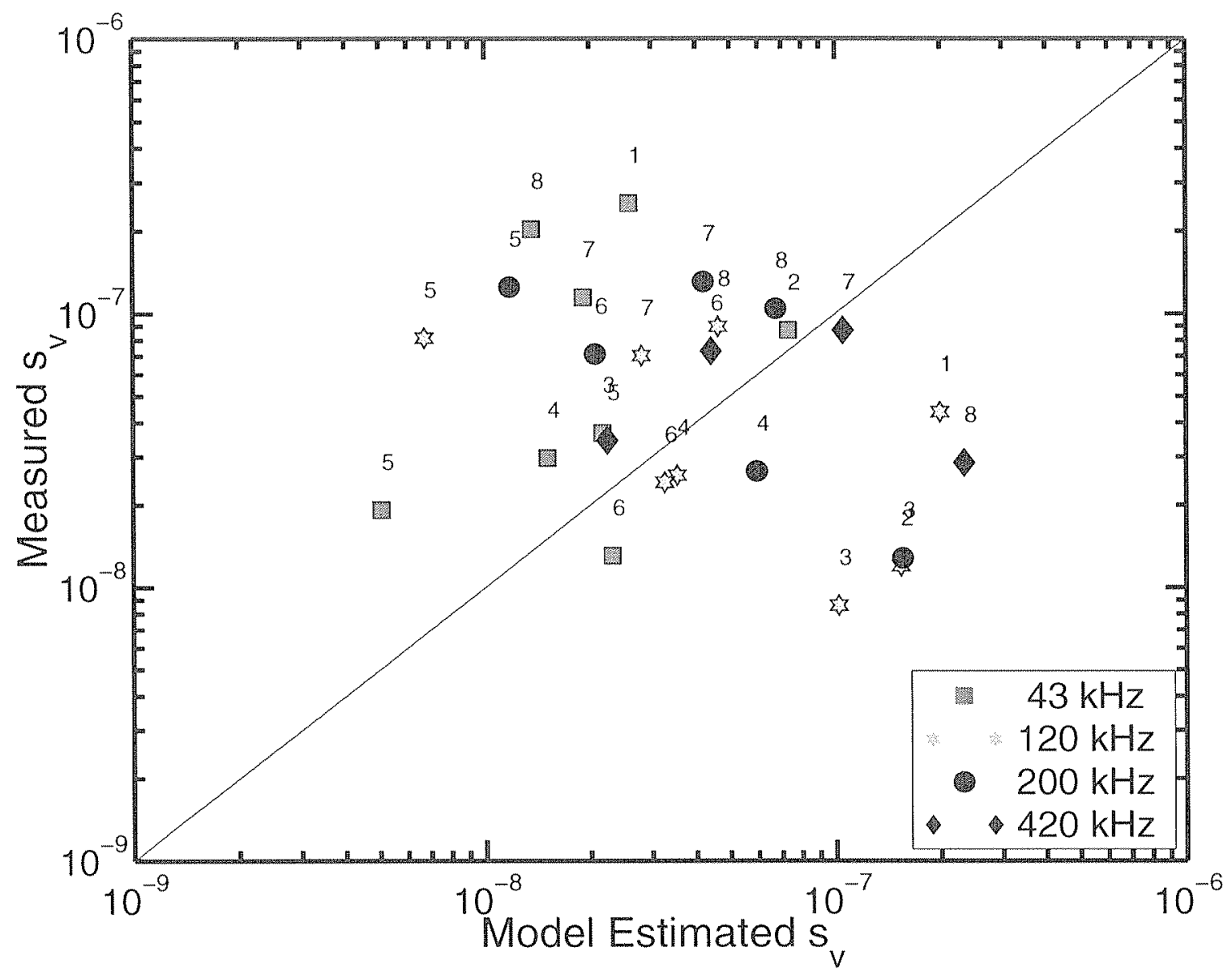

Figure A-13: Forward Problem calculations for scattering at each frequency (symbol color and shape) and net number (number above each symbol) for MOCNESS \#7. Predictions from a few nets overestimate the scattering, while most of the other predictions were underestimates. 

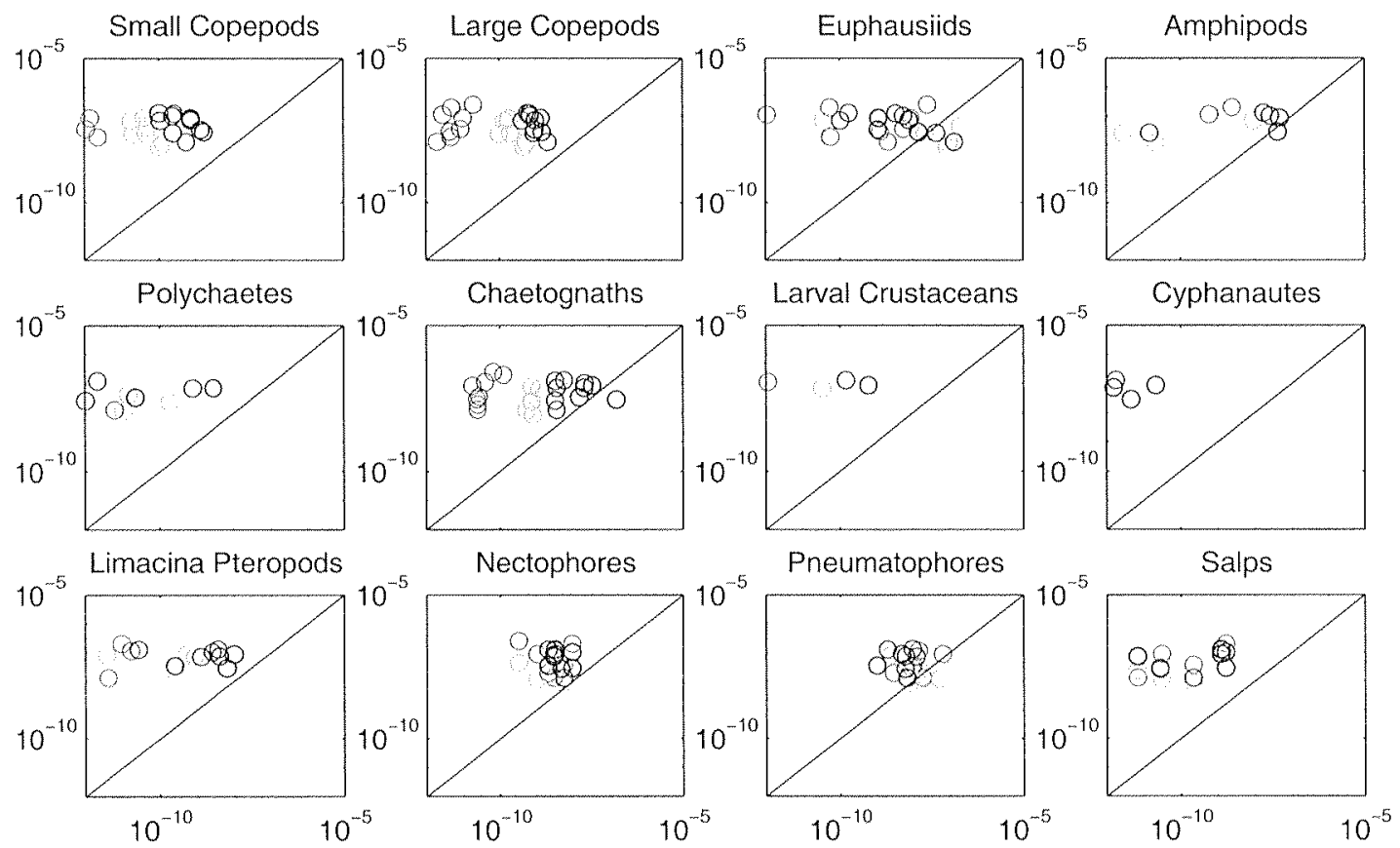

Figure A-14: Forward problem calculations for MOCNESS \#7 broken down for different taxonomic types. The horizontal axis in each plot is the predicted $s_{v}$, while the vertical axis is the measured $s_{v}$ by BIOMAPER-II. The data are expected to lie above the vertical line since any values on or below the line mean that all of the measured scattering is accounted for by that animal. Euphausiids, amphipods, chaetognaths, pteropods and siphonophore nectophores and pneumatophores were the principal contributors to the predicted scattering. 


\section{A.1.5 Prediction of $\epsilon$ Using Thorpe lengths}

The Thorpe lengths (Figure A-15) were found by averaging $L_{T}$ over twenty-eight different depth bins selected by hand that separated regions of high and low variability in temperature, salinity, or density.
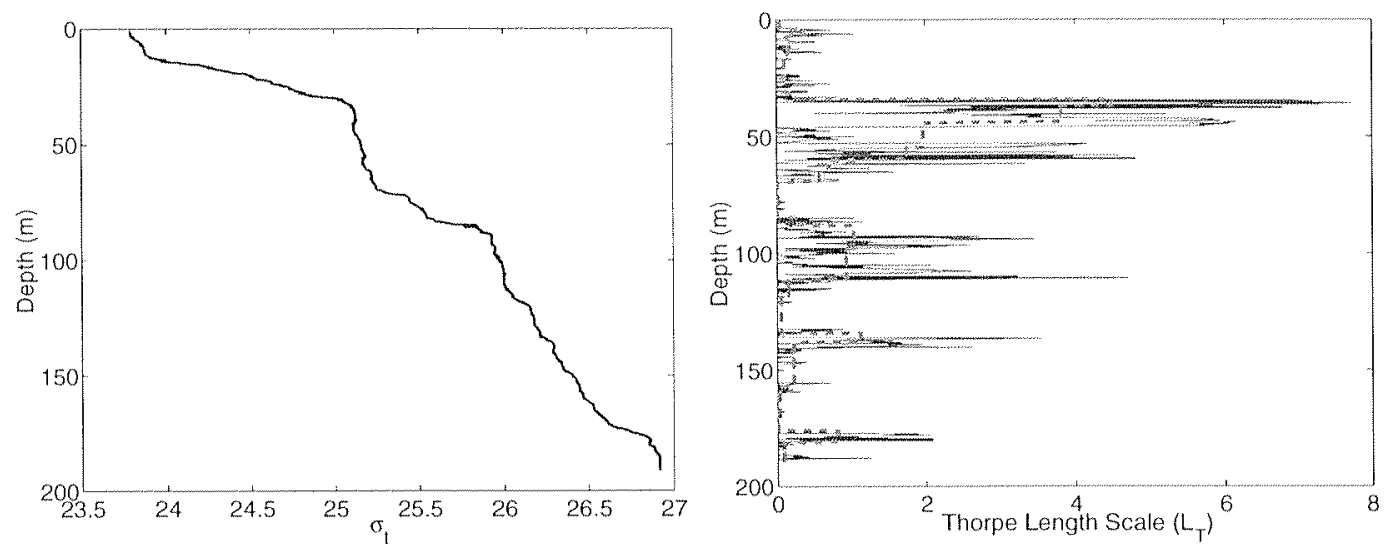

Figure A-15: Density profile from MOCNESS \#7 (left) and the calculated Thorpe lengths (right). The dashed line is the mean $L_{T}$ for various depth bins selected by eye.

Once the Thorpe length has been estimated, values for $\epsilon$ and $\chi$ can be found using equations 4.8, 4.9, 4.10, and 4.11. The resulting estimates (Figure A-16) show values of both $\epsilon$ and $\chi$ that are low in the near-surface regions (contrasting with the values found for MOCNESS \#9), with peaks in various regions throughout the water column (occurring at approximately 40,60, 90-100, 140, and 180 meters depth).

Once $\epsilon$ and $\chi$ are known, they may be used to predict the acoustic scattering contributions from microstructure. The microstructure scattering model can be used to find the values of $s_{v}$ for each of the frequencies that BIOMAPER-II uses (the $1 \mathrm{MHz}$ data are not analyzed due to its limited depth range). Levels of scattering comparable to that of dense assemblages of zooplankton are predicted in various regions of the water column by the microstructure scattering model (Figure A-17). Similar to the data from MOCNESS \#9, the predicted values are too large in some regions and would account for all of the measured scattering in the water column. 

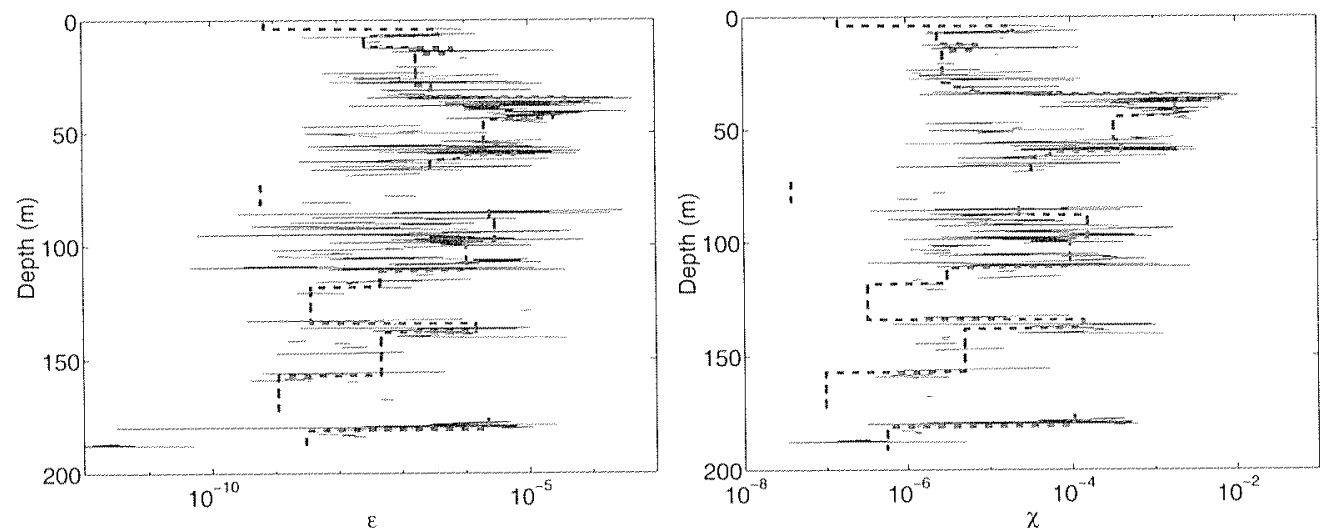

Figure A-16: Calculated values of $\epsilon$ (left) and $\chi$ (right) from hydrographic information from MOCNESS \#7 using equations 4.8,4.9,4.10, and 4.11. Dashed lines are the mean value for the depth bins selected for the Thorpe length analysis.

\section{A.1.6 Inversion of Acoustic Data for $\epsilon$}

Another method of estimating $\epsilon$ is to run the theoretical scattering model for a wide range of values of $\epsilon\left(\epsilon=10^{-10}-10^{-3}\right)$. This produces plots of $\sigma_{b s s}$ or $s_{v}$ versus depth versus $\epsilon$ and these plots can be made for a specific acoustic frequency, such as those used by BIOMAPER-II (Figure A-18).

If the differences in the measured and estimated scattering values from the Forward Problem calculations (Figure A-19) are assumed to be contributions to the scattering from microstructure, then this value of scattering can be inverted (using the output of the theoretical microstructure scattering model) for values of $\epsilon$, the dissipation rate of turbulent kinetic energy (Figure A-20). This method can only be used for regions where the measured scattering is larger than the estimated scattering, since if the measured scattering was smaller than the estimates then that implies that our zooplankton scattering models are inaccurate (to some extent). The estimates of $\epsilon$ are found for the depth ranges that correspond to the MOCNESS tows. There were low values $\left(10^{-9}\right)$ of turbulence near the bottom, and large values $\left(10^{-6}-10^{-4}\right)$ of turbulence in the near surface nets, which correspond to the depths that the acoustic scattering layers are found. 


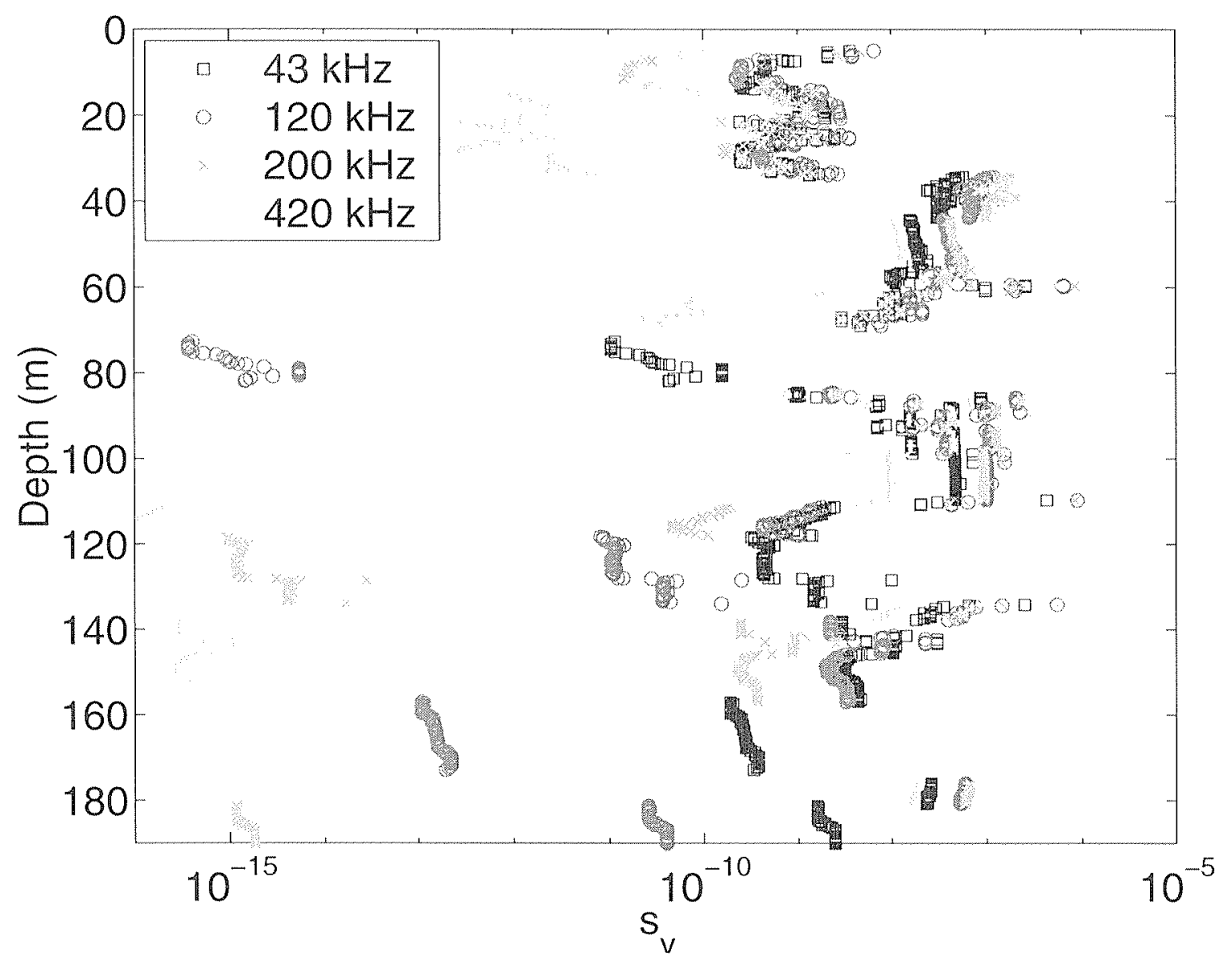

Figure A-17: Predicted levels of volume scattering from Seim's model and the hydrographic information from MOCNESS \#7. There is increased scattering from microstructure at the surface and in the regions that appear to be well mixed in the density profile. It should be noted that the largest amount of scattering is not always at the same frequency which suggests that the length scale of the microstructure or turbulence is varying throughout the water column as well. These values of volume scattering are likely to be too large since they would account for all of the scattering that was measured in the water column, this is attributable to our method of estimating $\epsilon$ which is an imperfect assumption. 

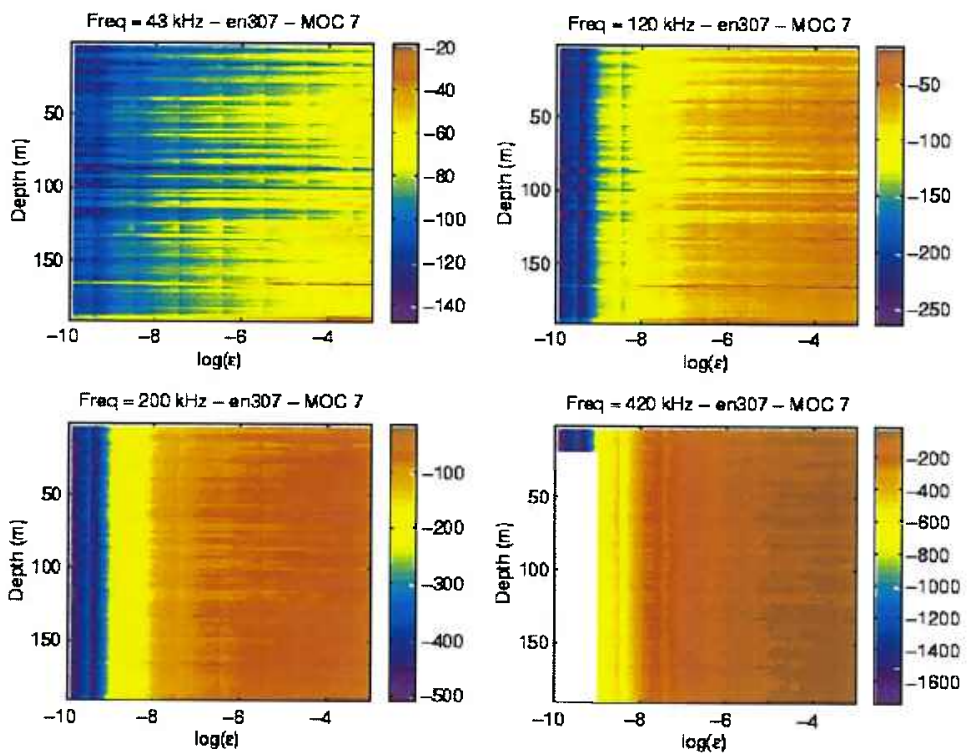

Figure A-18: Theoretical values of volume scattering strength from the Seim model (using hydrographic information from MOCNESS \#7) for the four frequencies used by BIOMAPER-II and a wide range of $\epsilon$ values. These data is used in the inversion of the "leftover" scattering data (once the biological caused scattering is accounted) to provide predictions of $\epsilon$ in the water column. 


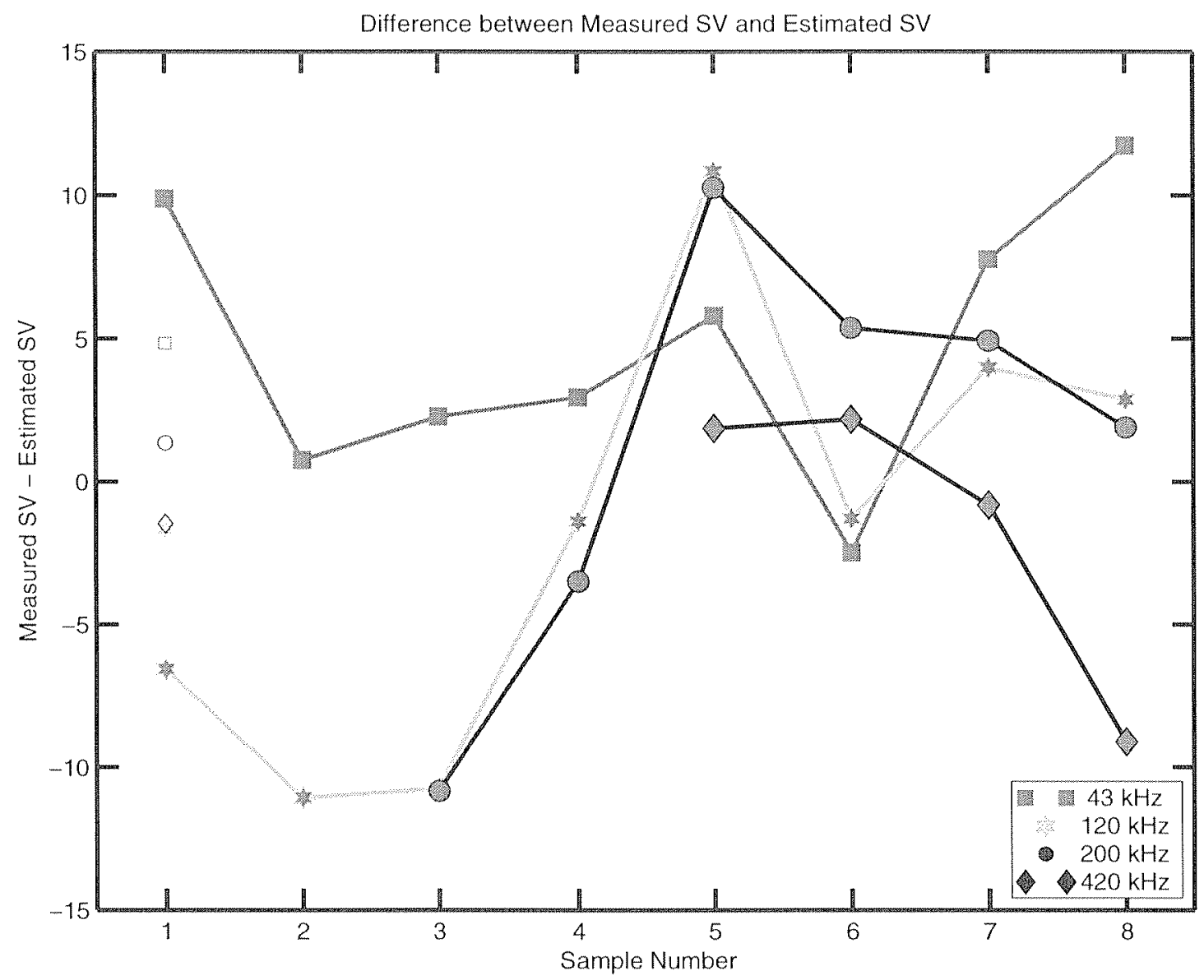

Figure A-19: Errors in the measured and predicted levels of volume scattering for MOCNESS \#7. The vertical axis is the difference between the measured volume scattering strength (from BIOMAPER-II data) and the predicted level of scattering (from theoretical scattering models and MOCNESS zooplankton and CTD data). The volumes are determined by where the MOCNESS nets were opened and closed. The horizontal axis is MOCNESS net number where \#1 is the deepest net and $\# 8$ is the near-surface tow. Values above 0 are underestimates and values less than 0 are overestimates by the FP predictions. The underestimated values are used in the inversion of acoustic scattering data for values of $\epsilon$. Mean errors for each frequency are shown on the left. 
Data from MOC $7 t(z)$ and $s(z)$ profiles

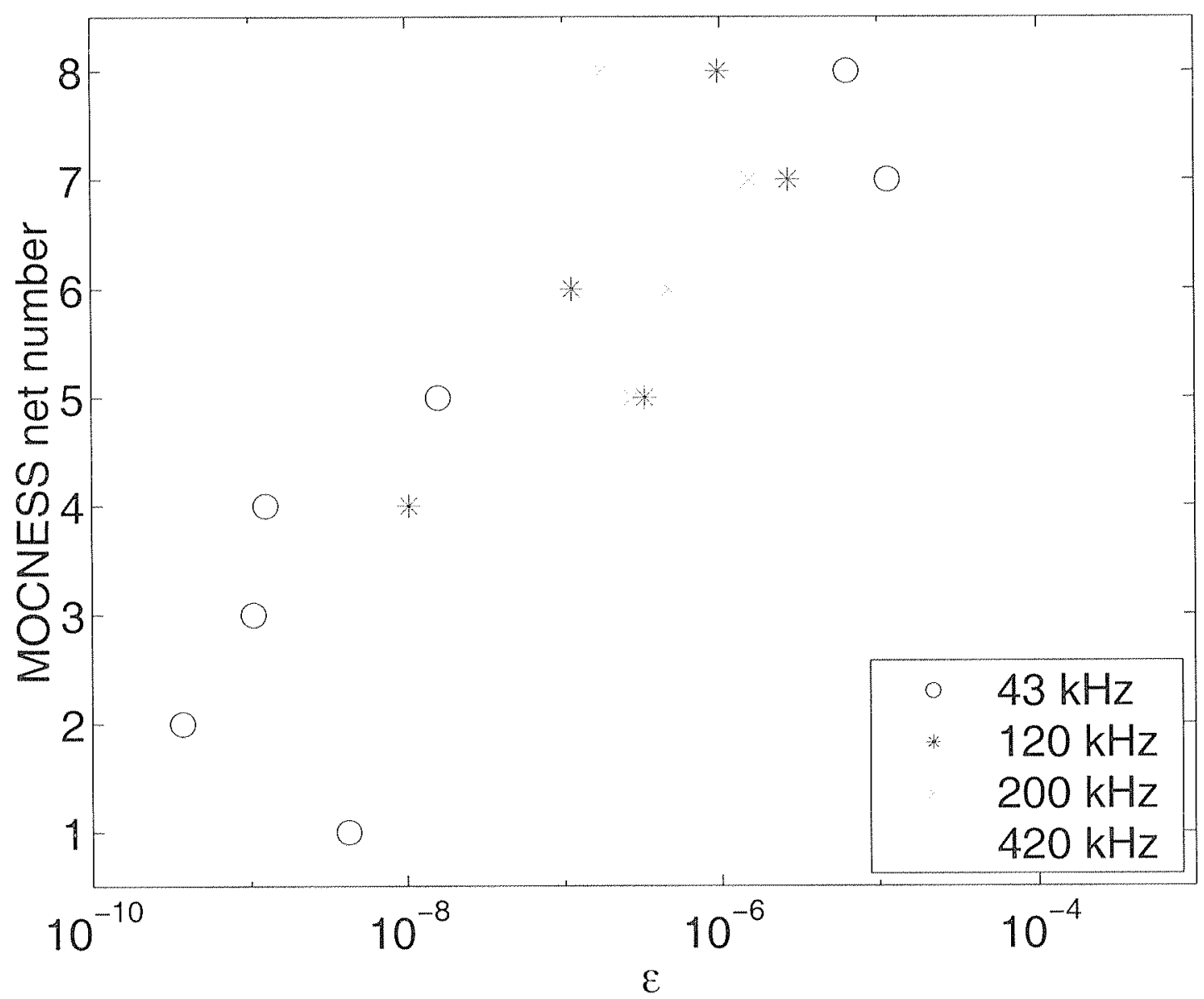

Figure A-20: Estimates of $\epsilon$ from the remaining measured acoustic scattering once biological sources have been accounted for from MOCNESS \#7. Large values of $\epsilon$ are found in the surface layer and are of the order of dissipation rates for an internal wave. Smaller values are found in the deeper waters where the internal wave was not seen to be propagating and are typical for open ocean turbulence. 


\section{A.1.7 Modified Forward Problem calculations}

With these new scattering models in place, the FP calculations were done again to assess the contributions that biological and physical processes make to the acoustic scattering. The modified FP predictions show a more equal distribution of overand under-estimates (Figure A-21). The taxonomic composition of the predicted scattering level shows that microstructure can contribute a substantial amount to the overall scattering (Figure A-22). Other changes due to the modified zooplankton scattering models is an increase in the contribution from copepods (particularly the large ones) and a change in the errors associated with each frequency and net (Figure A-23).

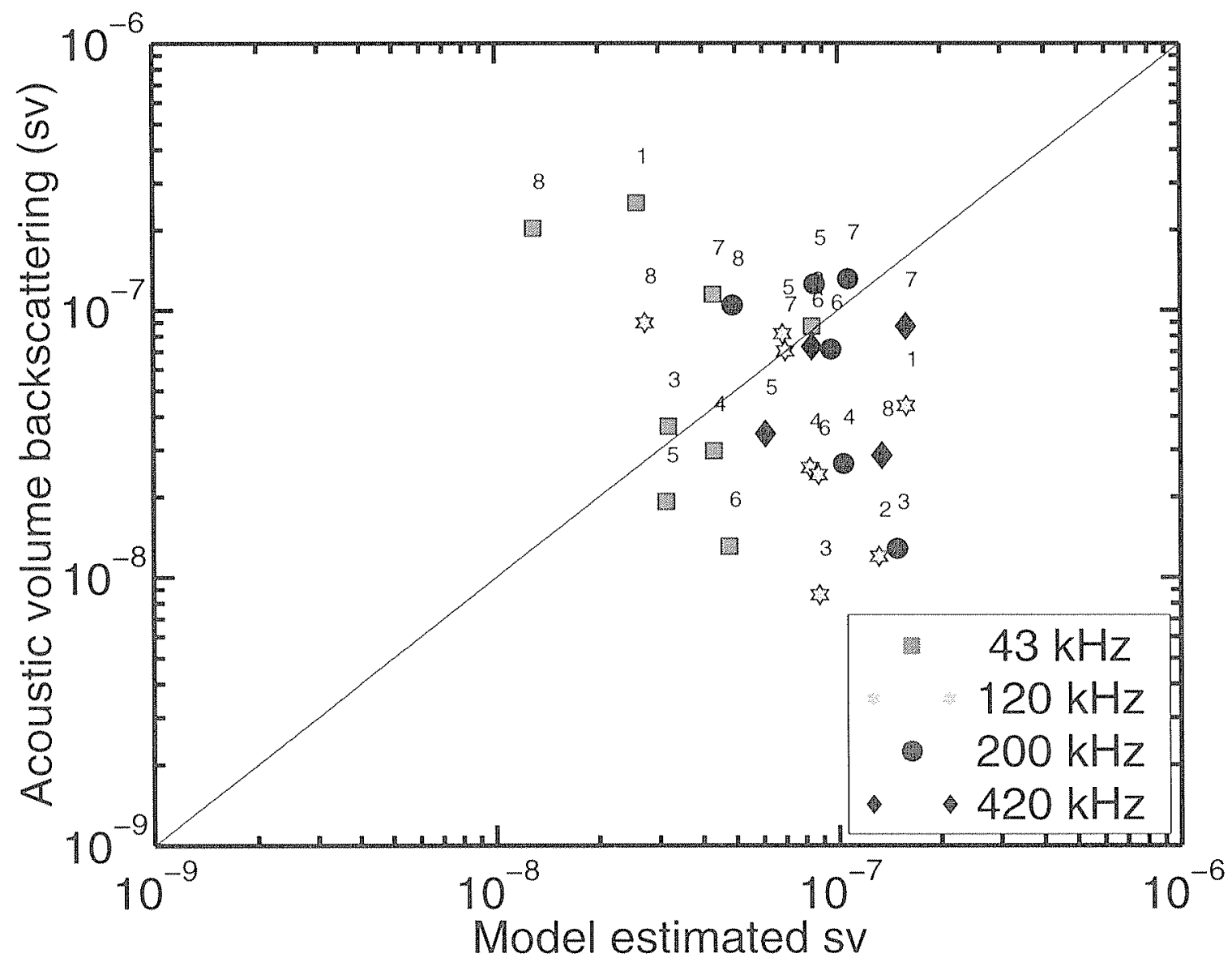

Figure A-21: Forward Problem calculations for MOCNESS \#7 including microstructure and the modified zooplankton scattering models. A more equal distribution of over- and under-estimates is clearly seen. 

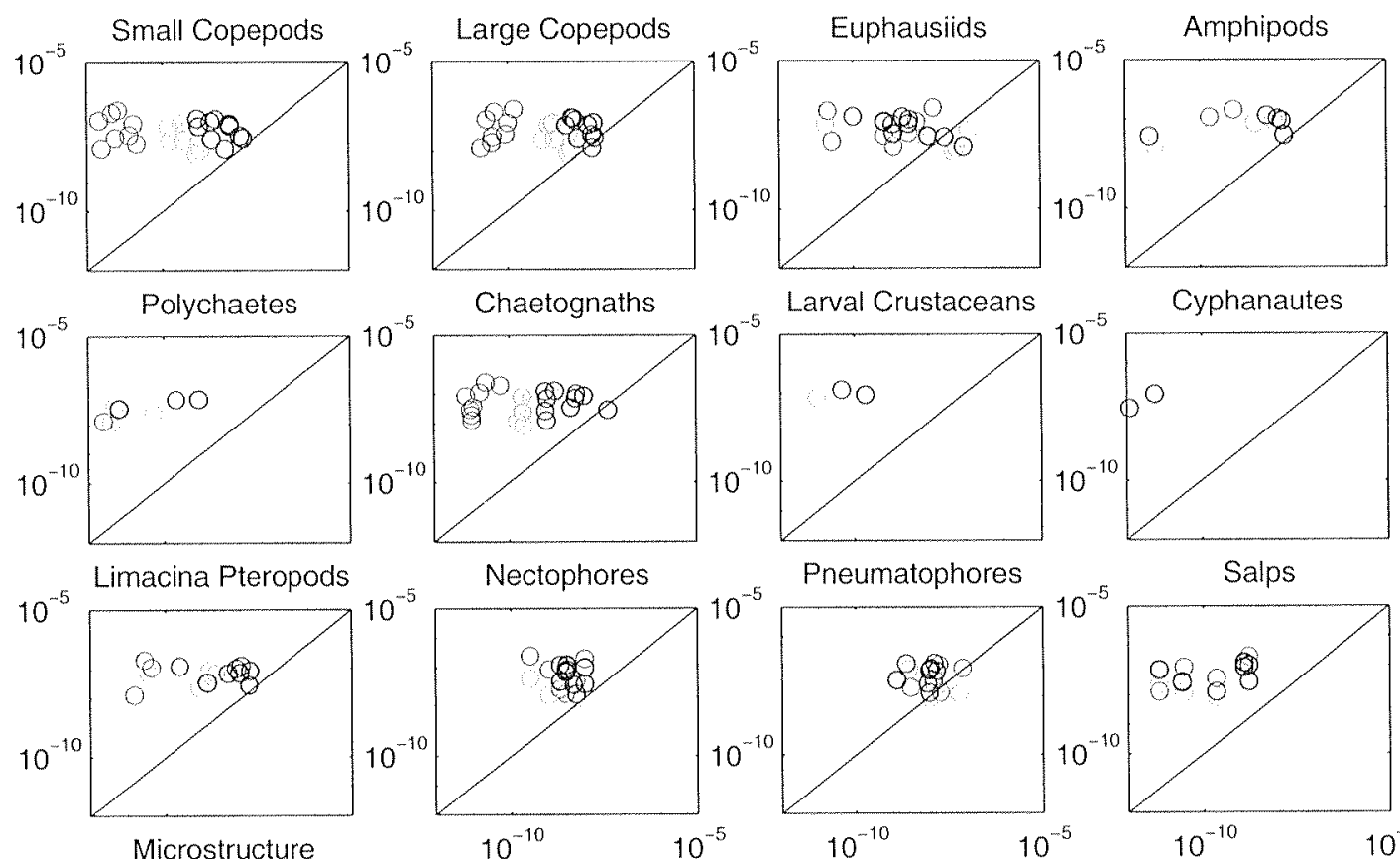

Pneumatophores

Salps
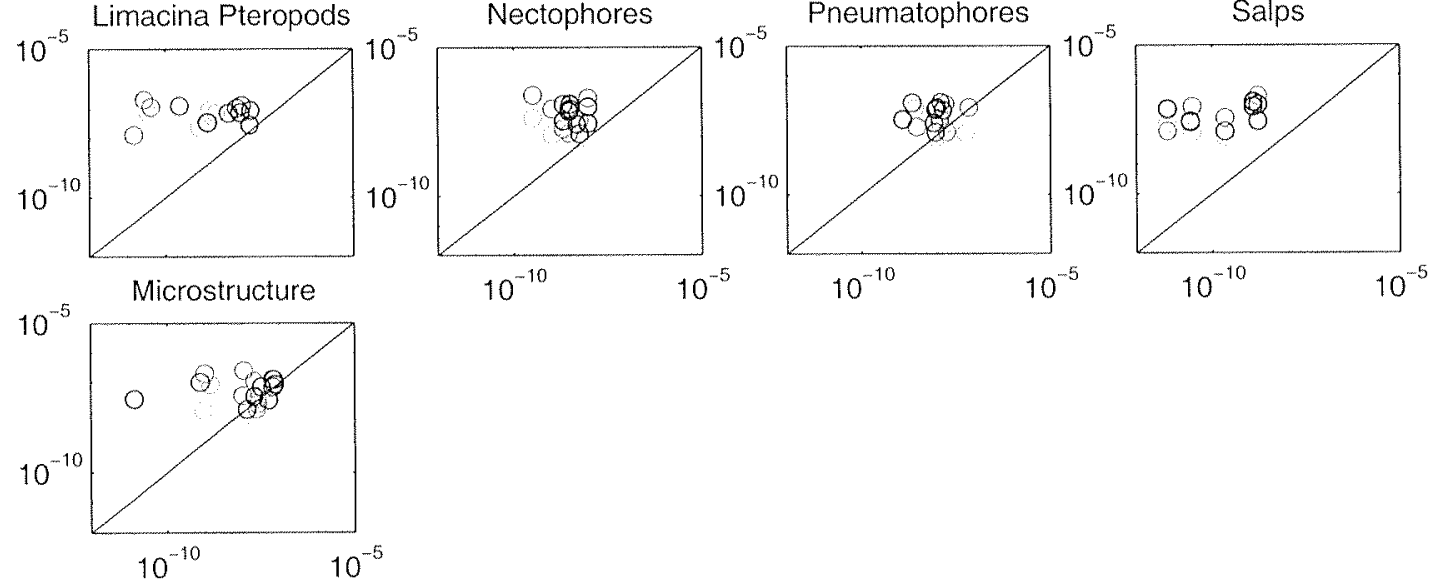

Figure A-22: Forward problem calculations using the modified zooplankton scattering models and including microstructure contributions for MOCNESS \#7 broken down for different scattering processes. The horizontal axis in each plot is the predicted $s_{v}$, while the vertical axis is the measured $s_{v}$ by BIOMAPER-II. The data are expected to lie above the vertical line since any values on or below the line mean that all of the measured scattering is accounted for by that process. Salps, euphausiids, amphipods, siphonophore nectophores, and microstructure were the largest predicted scatterers.

To quantify the relative errors of the three methods used in the FP calculations (original zooplankton scattering models, modified zooplankton scattering models, and inclusion of microstructure scattering model), the difference between the predicted and measured values of $S_{v}$ are shown for each net and frequency for MOCNESS tow \# 7 (Figure A-24). Generally all three methods have the same order of magnitude of error, however the first method (original zooplankton scattering models) tend to underestimate the amount of scattering while the final method (modified zooplank- 


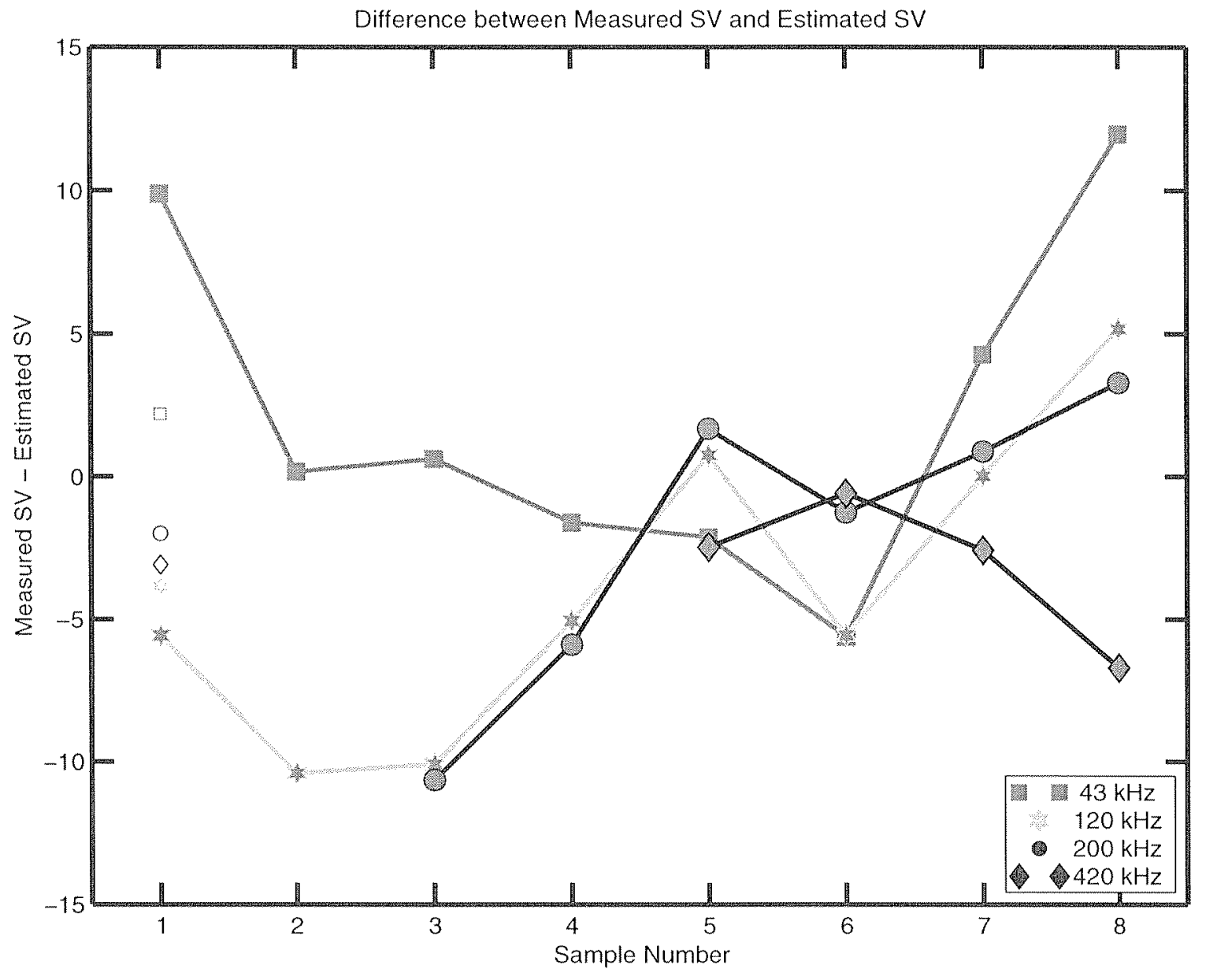

Figure A-23: Errors in the modified Forward Problem calculations for MOCNESS \#7 including microstructure and modified zooplankton scattering models. The vertical axis is the difference between the measured volume scattering strength (from BIOMAPER-II data) and the predicted level of scattering (from theoretical scattering models and MOCNESS zooplankton and CTD data). The volumes are determined by where the MOCNESS nets were opened and closed. The horizontal axis is MOCNESS net number where \#1 is the deepest net and \#8 is the near-surface tow. More overestimates are seen which is due to the large amount of scattering predicted by the microstructure scattering model. Mean errors for each frequency are shown on the left. 
ton scattering models and microstructure scattering models) tend to overestimate the measured amount of scattering. Errors range from $1-10 \mathrm{~dB}$. The data from MOCNESS tow \# 7 show more reduction in errors with the final method than the MOCNESS tow \#9 data.

While these data may indicate that the inclusion of turbulence as a contributor to the scattering in the ocean does not improve the accuracy of the FP calculations, we have hypothesized reasons why the turbulence values are too large, and believe that with proper measurements of the inputs into the scattering models that the overall error in the FP calculations would be reduced.

\section{A.1.8 Sources of Biomass and Acoustic Scattering}

The data from MOCNESS tow \#7 are quite different in the distribution and type of scattering sources (Figure A-25). The scattering from microstructure occurs in the mid-water depths while euphausiids are found deeper and copepods, chaetognaths, amphipods and other scatterers dominate the surface net. The strong scattering due to siphonophore pneumatophores is most evident at the lowest BIOMAPER-II frequency $(43 \mathrm{kHz})$ which agrees with the scattering models discussed in Chapter Three due to the low resonance frequency for scattering from the gas inclusion. 
(a)

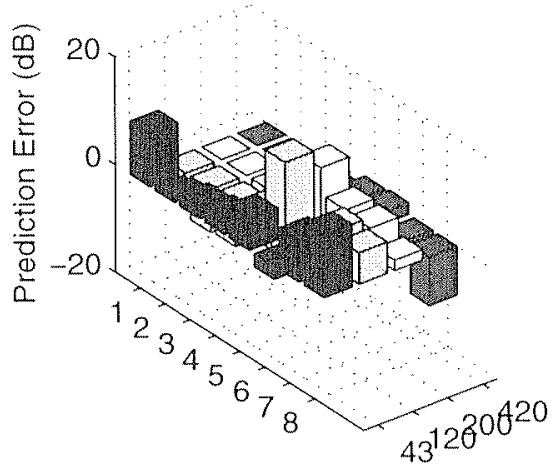

(d)

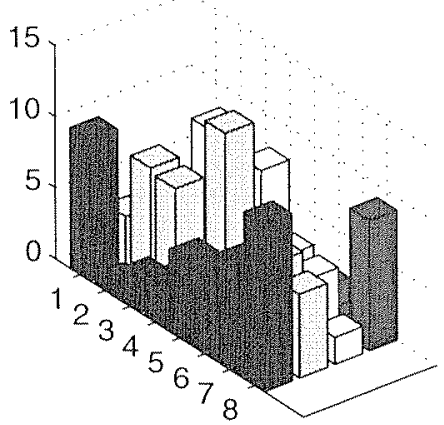

(b)

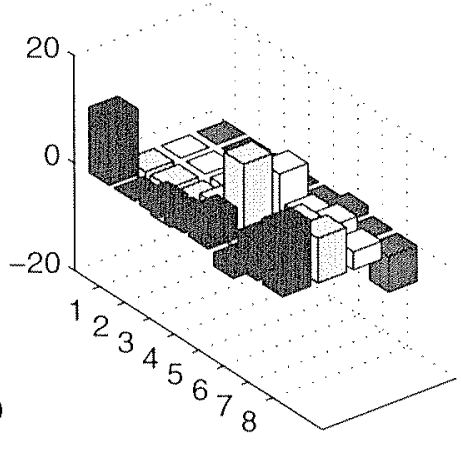

(e)

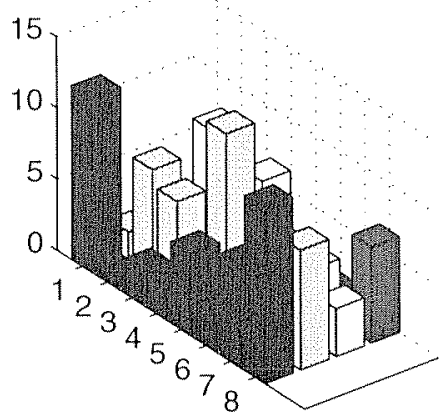

(c)

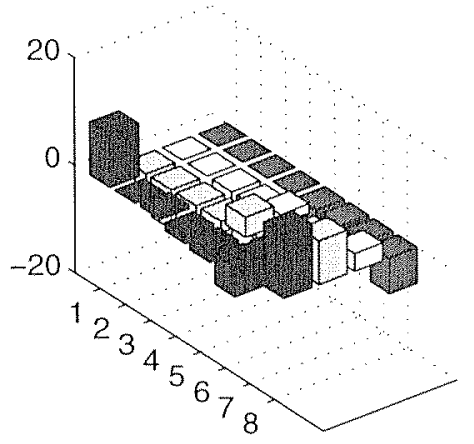

(f)

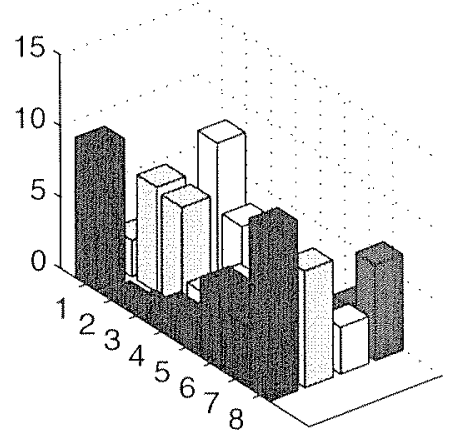

Figure A-24: Errors in the Forward Problem calculations for MOCNESS \#7 for the original zooplankton scattering models (left column), modified zooplankton scattering models (middle column) and the modified zooplankton and microstructure models (right column). The top row are the relative errors (where positive values are underestimates and negative values are overestimates by the FP calculations) and the bottom row is the absolute value of the errors. The numbers $1-8$ represent the MOCNESS net number, and the different BIOMAPER-II frequencies (in $\mathrm{kHz}$ ) are the other horizontal axis. The modified zooplankton scattering models combined with turbulence give slightly less error than the other methods. 


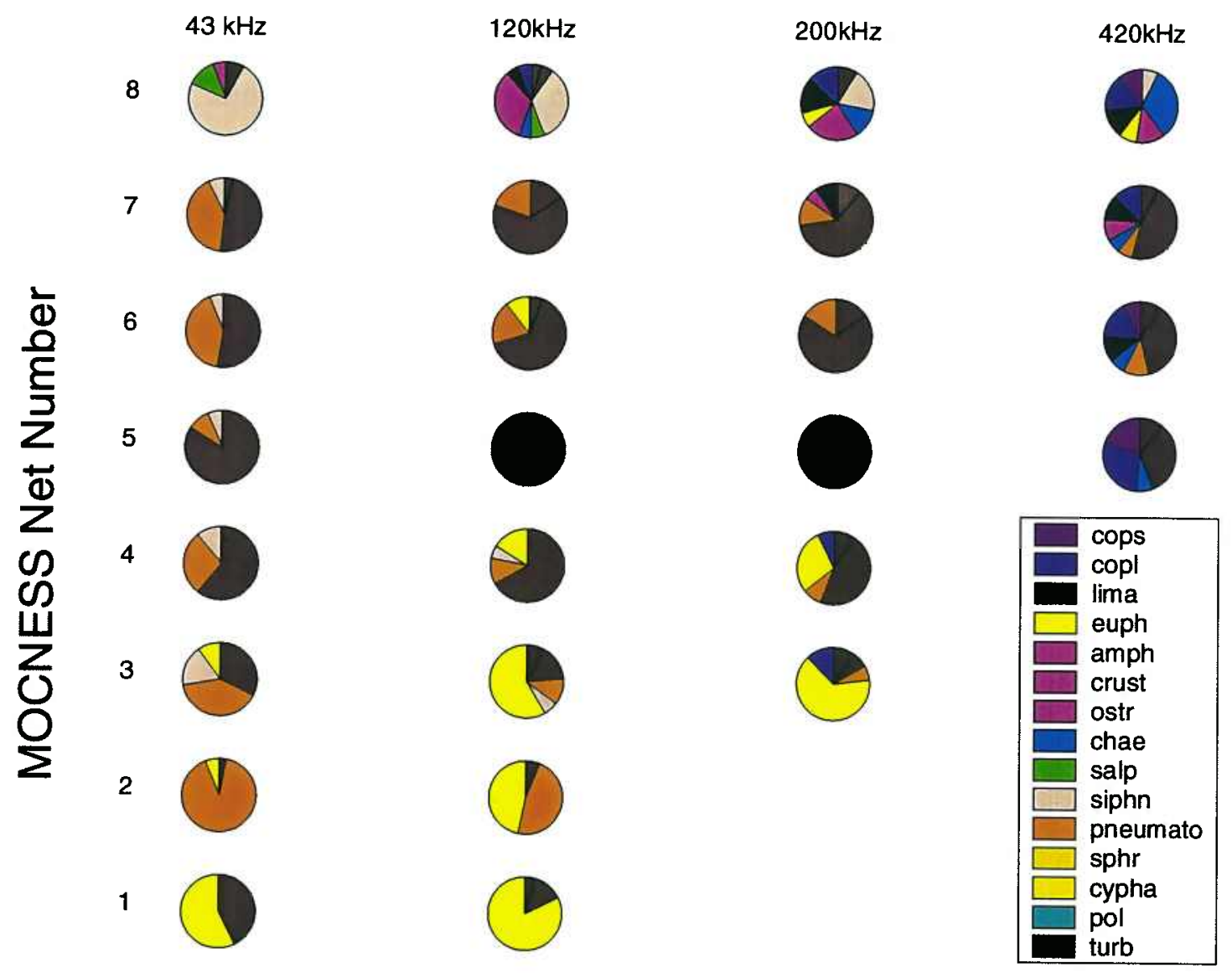

Figure A-25: Composition of the acoustic scattering predicted by Forward Problem calculations at four BIOMAPER-II frequencies using biological and hydrographic information from MOCNESS \#7. These data include contributions from microstructure. While a mixture of crustacean species cause the scattering in the surface net, microstructure contributes much of the scattering through out the water column, with euphausiids dominating the deeper nets. Siphonophore pneumatophores contribute enormous amounts of scattering in the middle and deep water column although only at lower frequencies. With increasing acoustic frequency, copepods become larger contributors to the overall predicted scattering level. 
If microstructure is excluded from the FP calculations then the water column appears to be dominated by copepods and assorted other scatterers near the surface, siphonophore scattering occurs apparently throughout the water column, and scattering from euphausiids was mostly found in the deepest waters. The biomass calculations and composition from the net tow (Figures A-7 and A-8) show that copepods were found throughout the water column with some euphausids present down deep. More importantly, siphonophores were not collected to any extent by the net tows, yet the ones that were collected would account for a large amount of the observed scattering. The biomass plot shows a surface layer peak along with an even greater amount of animals in the lowest three nets $(\# 1-3)$. Again, these data show that scattering contributions from microstructure MUST be taken into account to accurately interpret acoustic scattering data. 


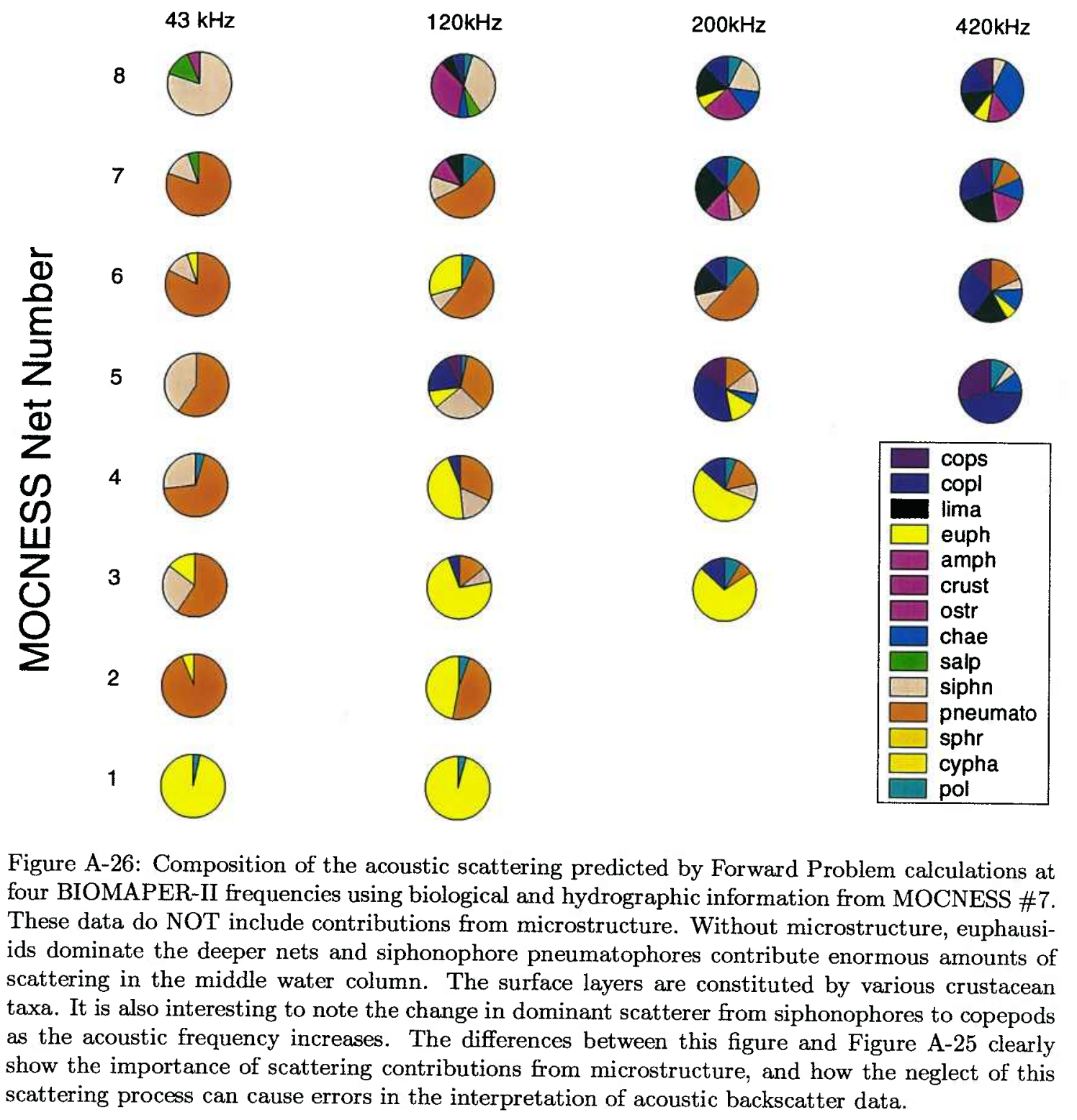


The previous two figures show clearly how different frequencies of sound can better detect different types of animals. With different acoustic frequencies the number of animals in each net does not change, however the relative proportion that the different animals make up of each "pie" in these figures is related to their acoustic scattering function. Small animals, such as copepods, are not "seen" as well at lower frequencies as they are at the higher frequencies, thus their share of the pie increases with frequency. Siphonophore pneumatophores have the opposite trend, due to a fairly flat spectral scattering curve except for a low frequency $(\sim 10-20$ $\mathrm{kHz}$ ) resonance peak in the spectra. Thus these animals contribute more at the lower frequencies. These figures show the problems that can result in trying to interpret single frequency acoustic backscatter data from assemblages of different animal types.

Finally, the accuracy of forward problem predictions (both with and without contributions from turbulence) with acoustic measurements is examined (Figure A-27). The largest differences between the predictions with and without microstructurecaused acoustic scattering occur in the middle of the water column (nets \#4 - 7) which is where predictions of scattering from microstructure are the largest. A comparison of Figures A-27 and A-7 shows that these same nets have the lowest amount of biomass, even though they have comparable amounts of acoustic scattering energy. Thus the contributions from microstructure scattering occur where the biological contributions are lowest due to low amounts of biomass. This information can not be determined by solely examining the acoustic scattering plot where there appears to be uniform scattering throughout the entire water column. Therefore contributions from microstructure must be accounted for if acoustic scattering data are to be properly converted to estimates of biomass.

However if the relative contributions from each scattering source are identified from the FP calculations, then the percentages of scattering from physical and biological sources is known. This can be combined with the measured scattering from the BIOMAPER data to produce a depth profile of biologically-caused acoustic scattering (Figure A-28). If physical scattering contributions are removed from the acoustic 


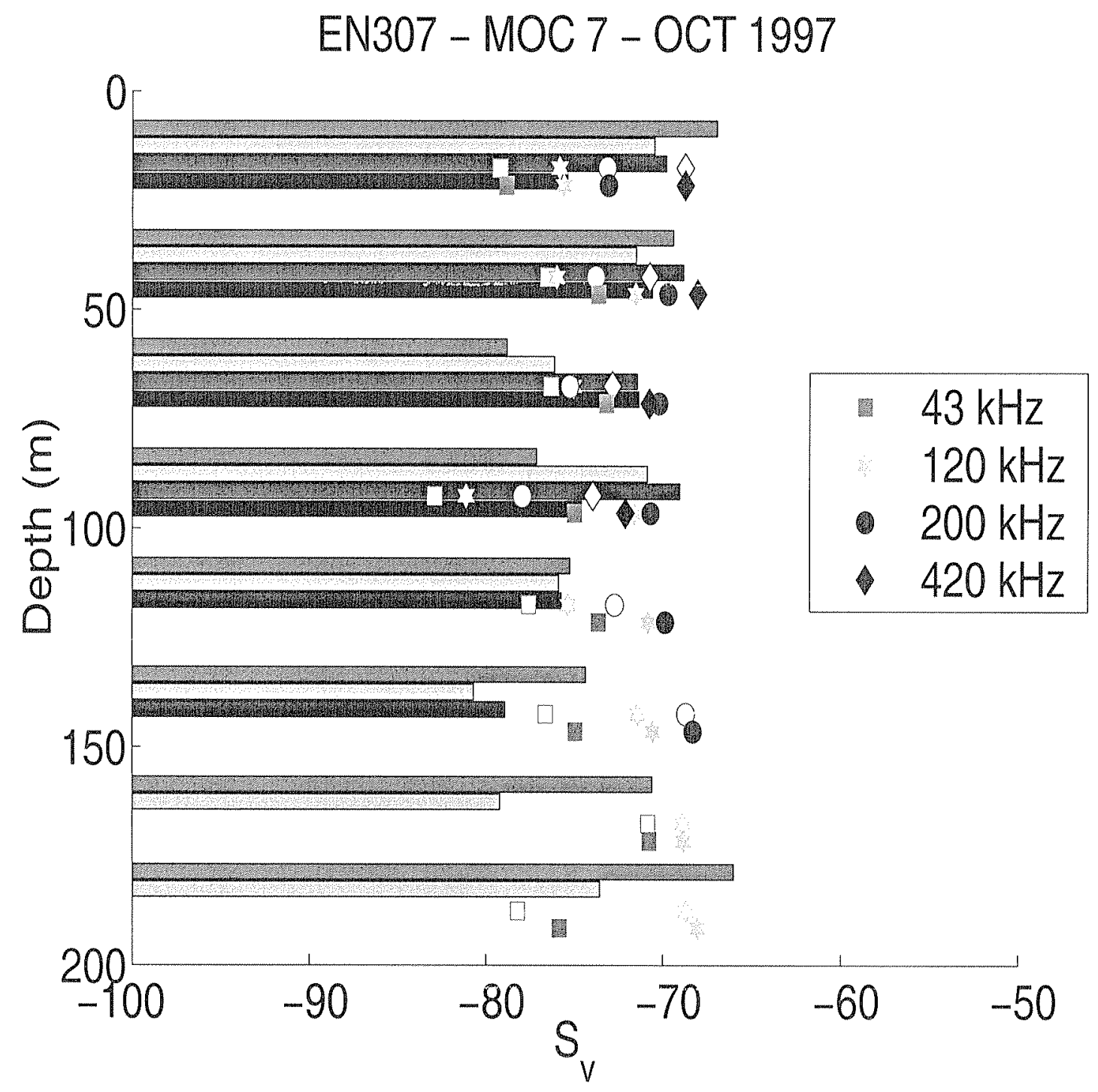

Figure A-27: Measured values of volume scattering (filled in bars) for each frequency during MOCNESS \#7 compared with predictions from FP calculations both with (filled in symbols) and without (empty symbols) scattering contributions from microstructure. The predictions are plotted below the corresponding measured values. 
data then the volume scattering coefficient becomes a much more accurate measure of biomass in the ocean (compare Figures A-28 and A-27 with Figure A-7). While there are still some discrepancies between the measured scattering and the biomass profiles, the overall trends and shape are correct. This shows that accounting for scattering contributions from physical processes is vital to properly interpret acoustic scattering data for information about biomass. 


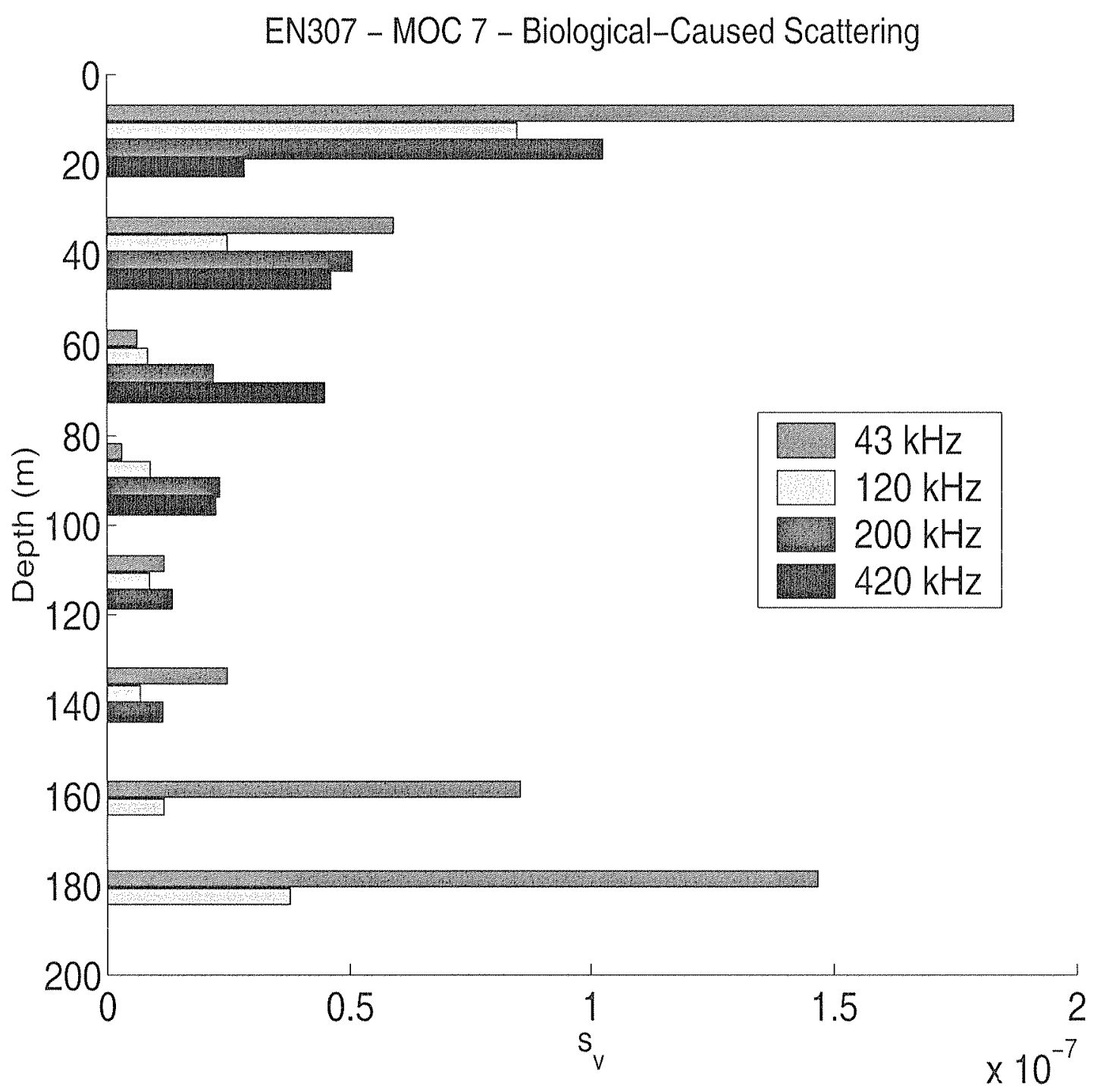

Figure A-28: Predicted values of volume scattering coefficient from biological sources of scattering for each frequency during MOCNESS \#9. There is a strong agreement between these data where scattering contributions from physical sources are removed and the depth profile of zooplankton biomass. 


\section{Appendix $\mathbb{B}$}

\section{Qualitative Analysis of VPR and Acoustic Scattering Data from the Internal Wave Survey}

The Video Plankton Recorder (Davis et al., 1992) on BIOMAPER-II was designed to provide quantitative ground-truthing information on the abundance and type of zooplankton found in the water column. Unfortunately, the video sampling volume used on the initial cruise (Endeavor 307) was too small, and thus not enough images of zooplankton were collected to fully quantify the distributions of various taxa. However the video images were identified (by hand) and the numerical density of different taxa are shown here with the $120 \mathrm{kHz}$ record for each of the three transects through the internal wave. BIOMAPER-II was at different depths during each transect so the interpretation of these plots is somewhat difficult. The VPR data was collected and processed by Mark C. Benfield.

\section{B.1 Transect One - Above the Internal Wave}

During the first pass through the internal wave, BIOMAPER-II was towed at the surface ( $\sim 5$ meters depth). The ESS and VPR data (Figures B-1 and B-2) do not 
show much correlation with the acoustic record. This is not surprising since the VPR and ESS system would only sample a tiny fraction of the internal wave (the upper layer barely grazes the blacked out region representing BIOMAPER-II's acoustic near-field region). Salps are detected by the VPR in this surface layer, but not in any of the other passes through the internal wave. This agrees with the MOCNESS \#9 data where salps dominated the surface layers but were not found anywhere else in the water column.
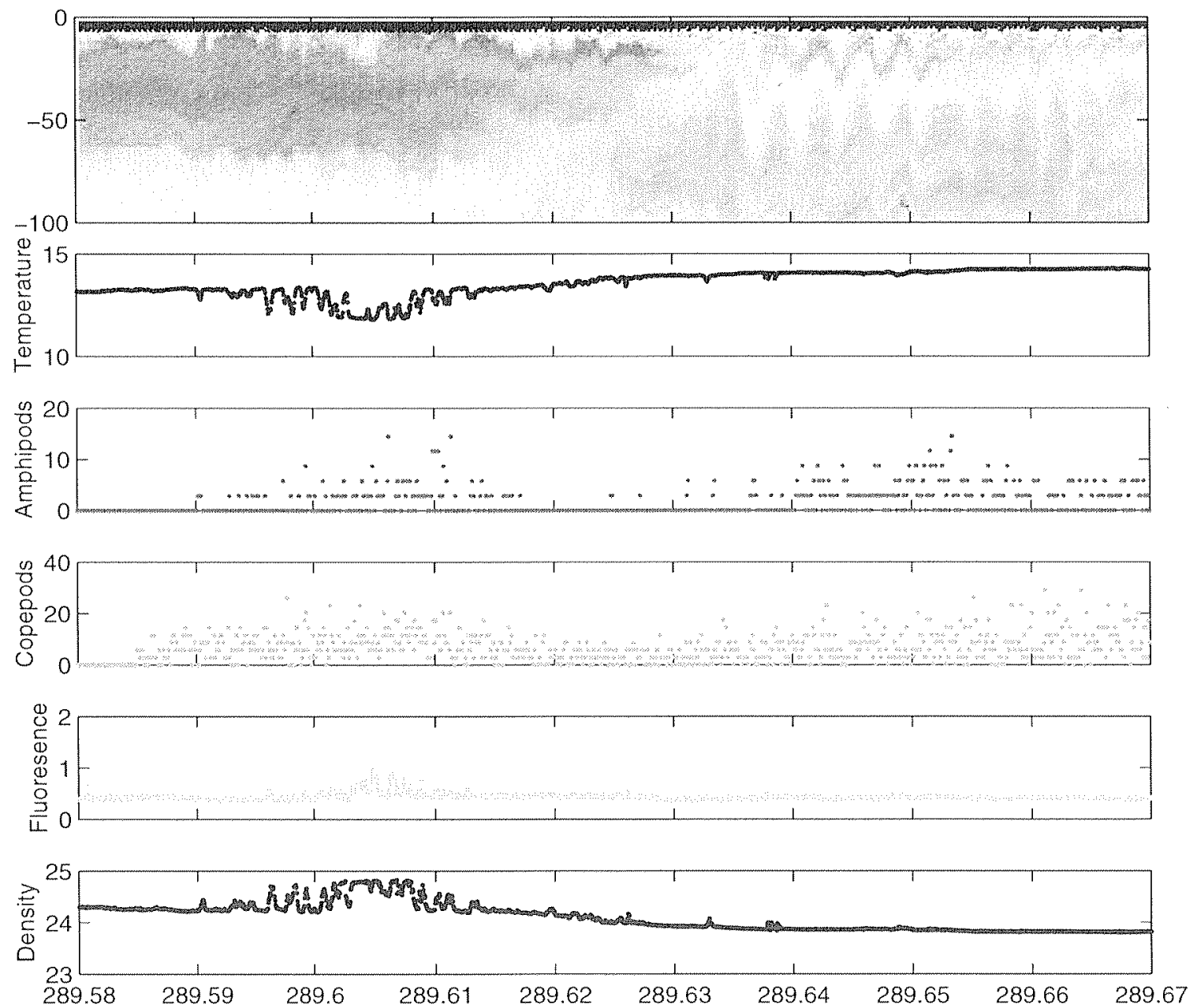

Figure B-1: $120 \mathrm{kHz}$ acoustic volume scattering for transect \#1 through the internal wave field along with VPR estimates of copepod and amphipod density and temperature, salinity, fluorescence, and density from the BIOMAPER-II ESS system. 

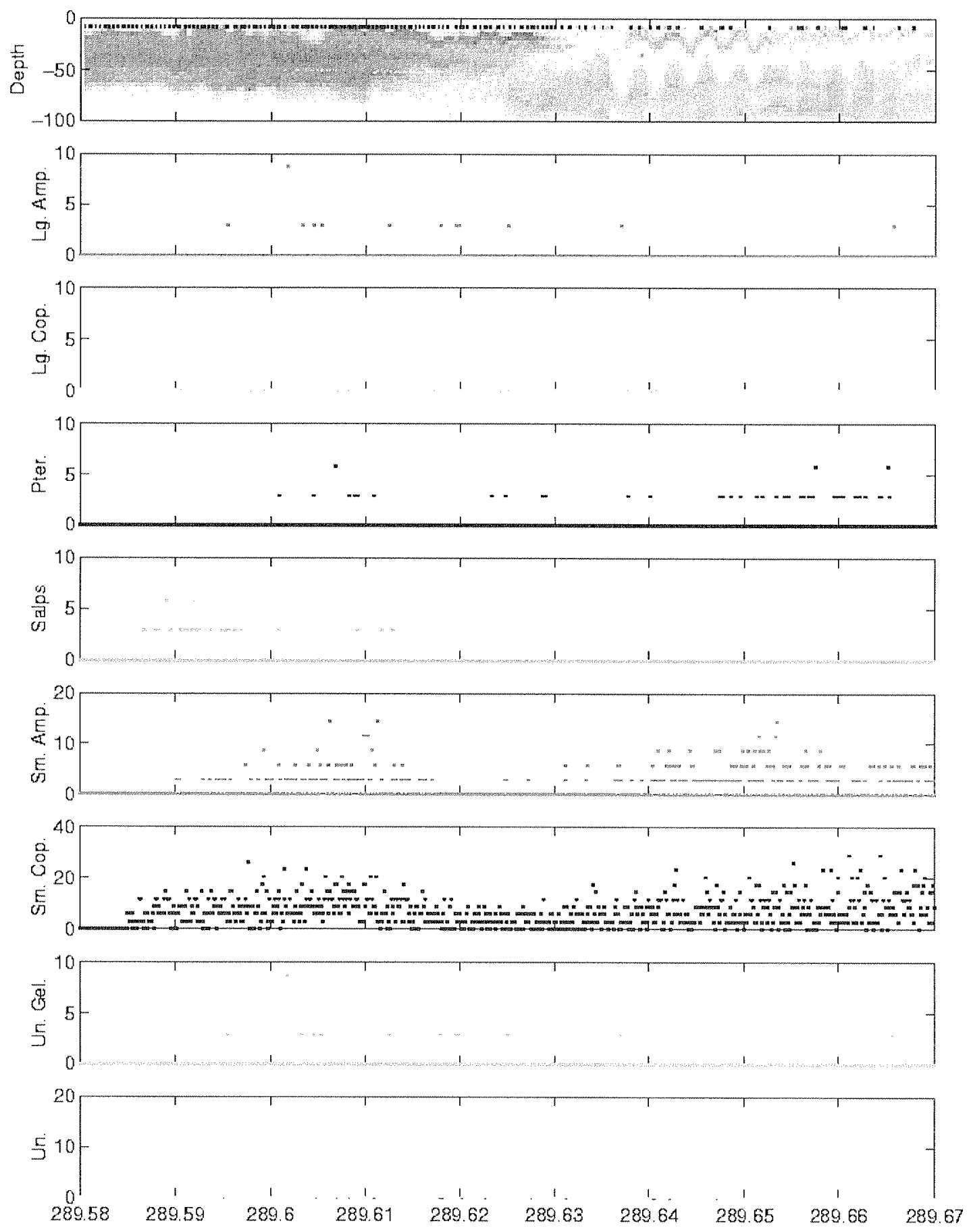

Figure B-2: $120 \mathrm{kHz}$ acoustic volume scattering for transect \#1 through the internal wave field along with VPR estimates of (top to bottom) large amphipod, large copepod, pteropod, salp, small amphipods, small copepods, unidentified gelatinous animals and unidentified animals. 


\section{B.2 Transect Two - Below the Internal Wave}

On the next pass through the internal wave, BIOMAPER-II was towed at a depth of $\sim 50$ meters. The ESS and VPR data (Figures B-3 and B-4) do not show much correlation with the acoustic record. The internal wave is not as clearly seen in the acoustic record although there are some sinusoidal variations seen between yearday $289.70-289.72$ which correspond to variations in the density measured by the ESS system on board BIOMAPER-II. Animal abundance is somewhat periodic, but there are not enough data to determine if they are correlated with the acoustic record. It is quite possible that our transect did not intersect the internal wave as cleanly as the previous one, or that the ship "broke" the wave up when it passed over it.

\section{B.3 Transect Three - In the Midst of the Internal Wave}

On the final pass through the internal wave, BIOMAPER-II was towed at a depth of $\sim 25$ meters. The acoustic record shows a more wave-like image than the previous pass which demonstrates that towing an instrument through a moving target (the internal wave) is not a simple task. The ESS and VPR data (Figures B-5 and B-6) show fairly high correlation with the acoustic record. The temperature and density data appear to track sinusoidal variations that are seen in the acoustic record. The VPR data appears to also track some of this sinusoidal behavior, unfortunately a missed VPR tape change occurred at yearday 289.84 so there is no animal abundance information from that point on. 

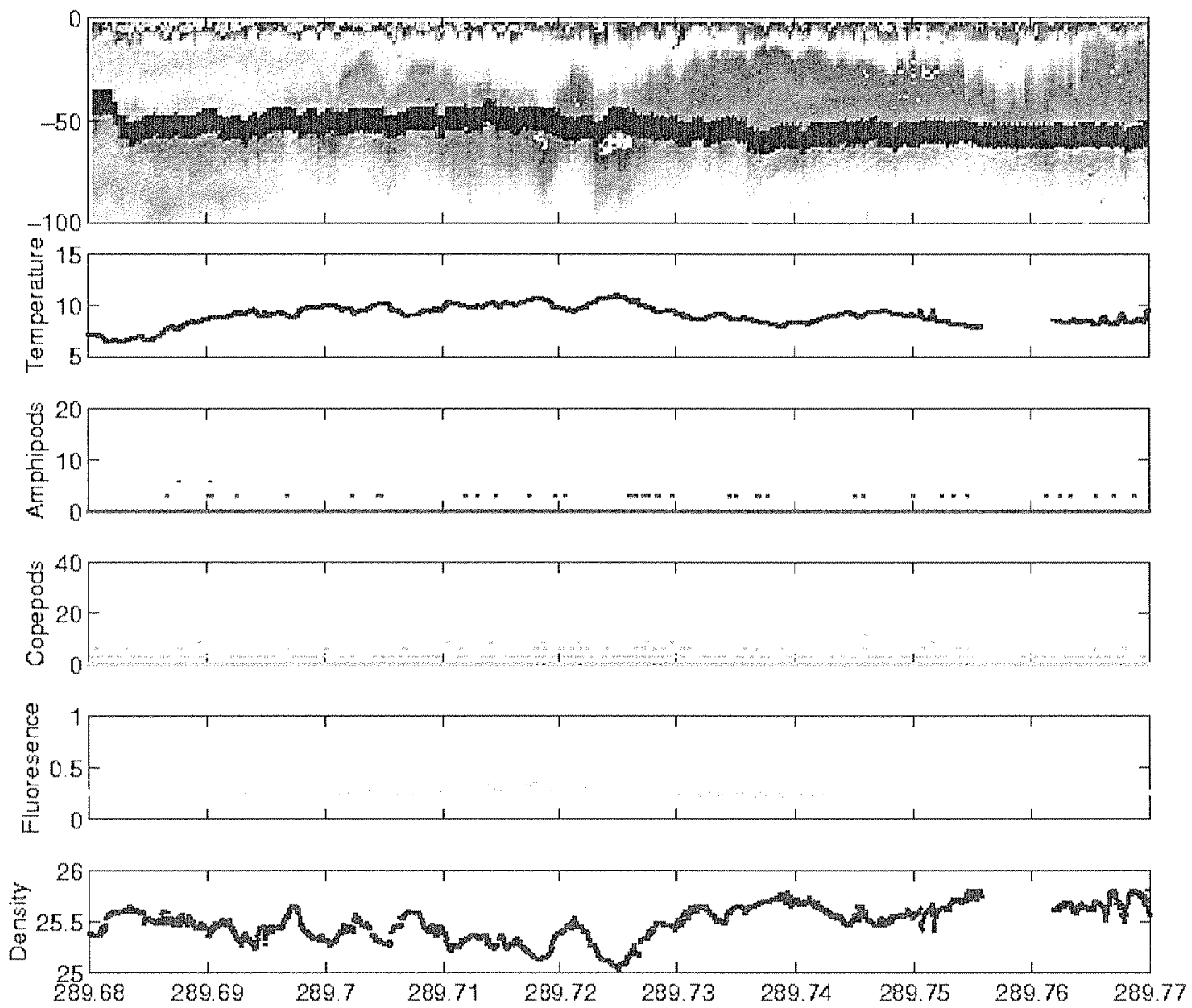

Figure B-3: $120 \mathrm{kHz}$ acoustic volume scattering for transect \#2 through the internal wave field along with VPR estimates of copepod and amphipod density and temperature, salinity, fluorescence, and density from the BIOMAPER-II ESS system. 

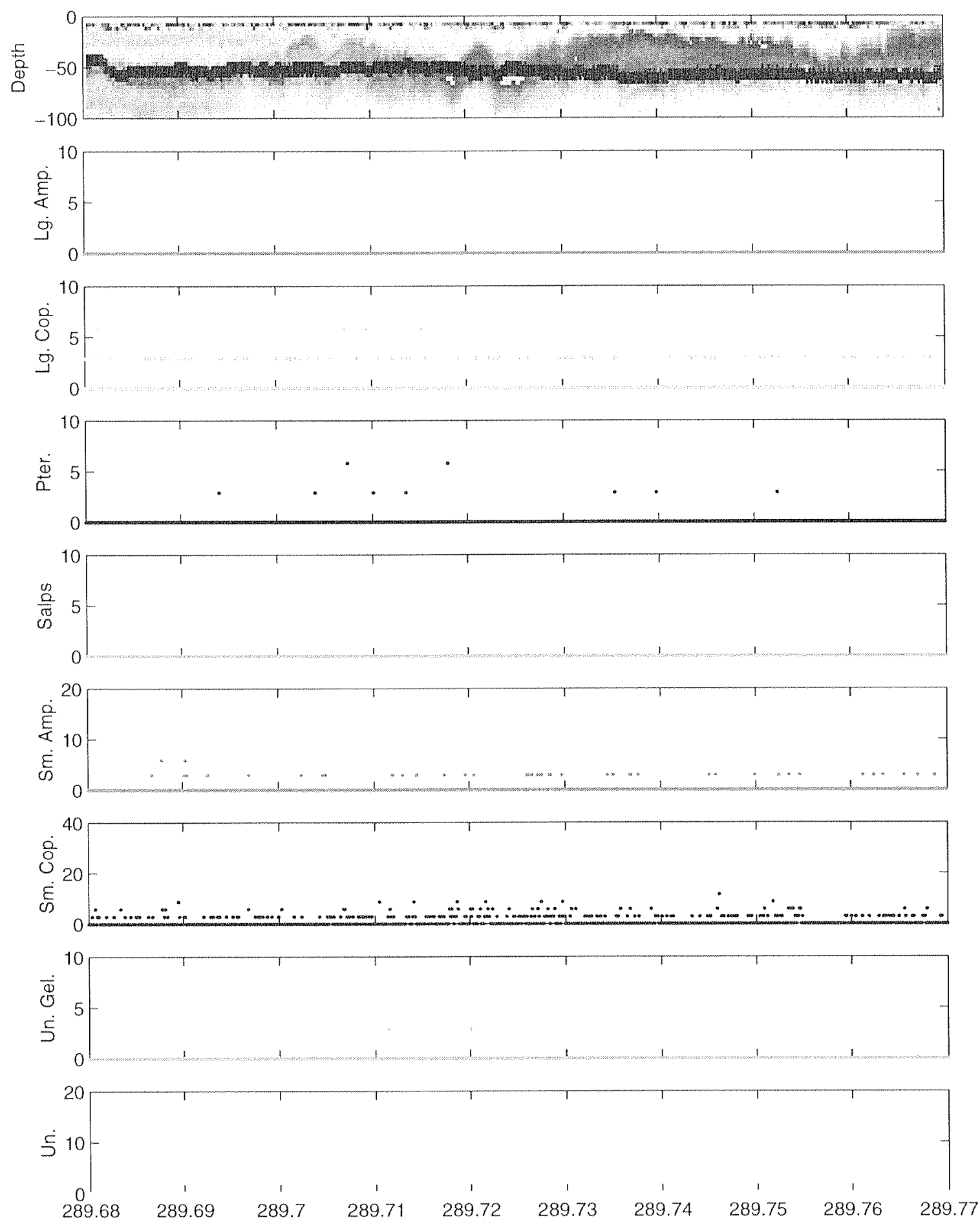

Figure B-4: $120 \mathrm{kHz}$ acoustic volume scattering for transect \#2 through the internal wave field along with VPR estimates of (top to bottom) large amphipod, large copepod, pteropod, salp, small amphipods, small copepods, unidentified gelatinous animals and unidentified animals. 

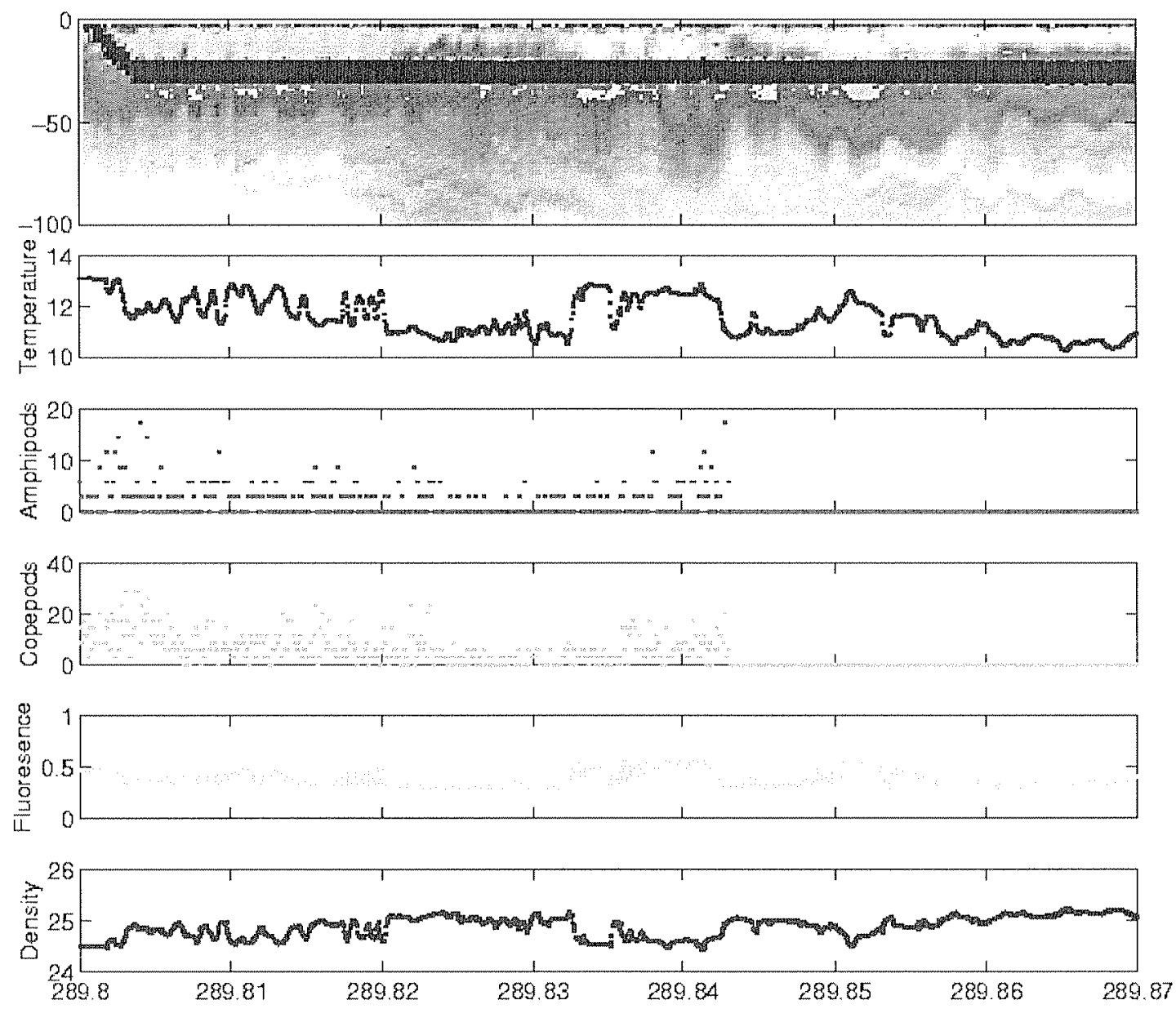

Figure B-5: $120 \mathrm{kHz}$ acoustic volume scattering for transect \#3 through the internal wave field along with VPR estimates of copepod and amphipod density and temperature, salinity, fluorescence, and density from the BIOMAPER-II ESS system. Animal abundance data were not collected after yearday 289.84 due to a missed tape change. 

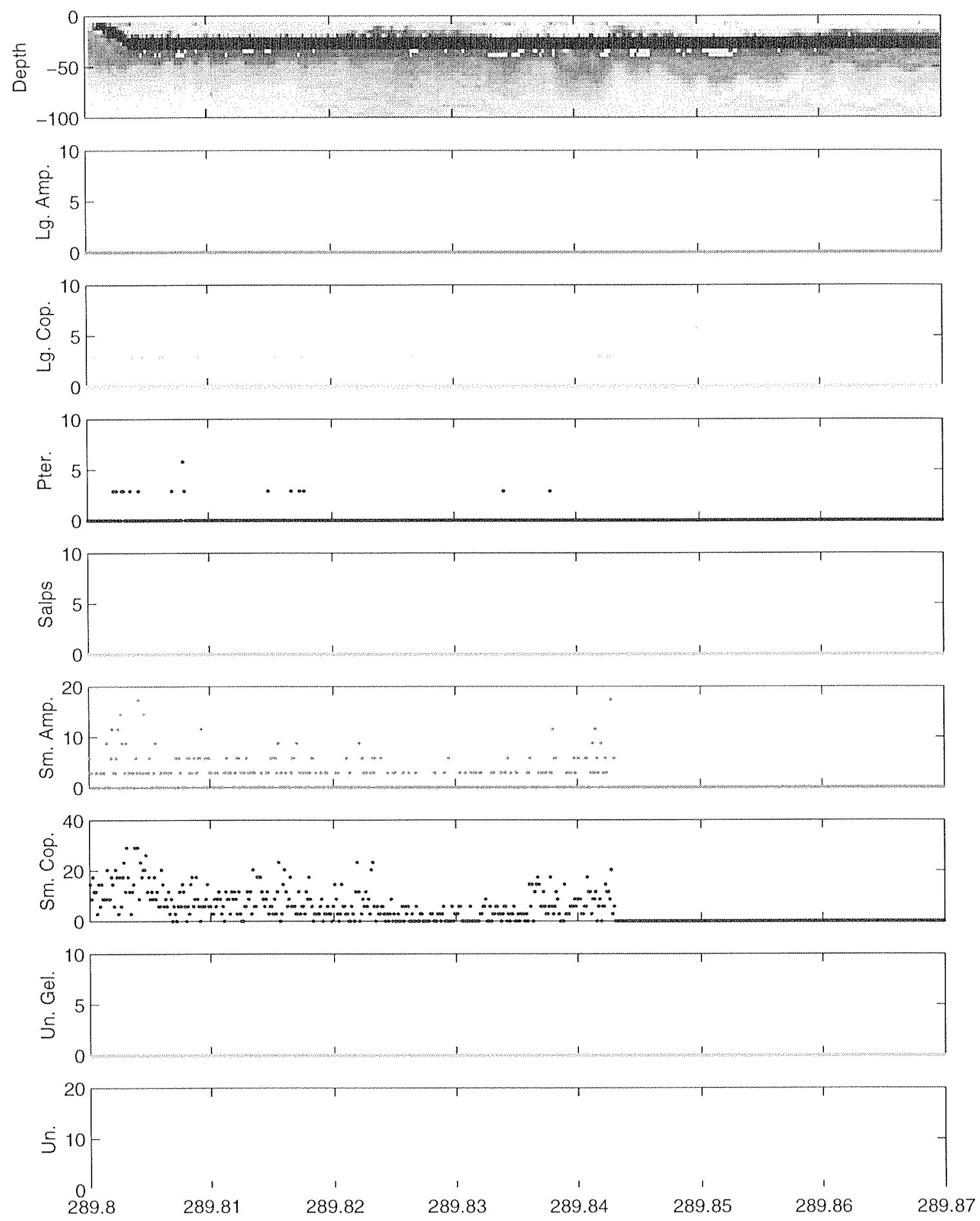

Figure B-6: $120 \mathrm{kHz}$ acoustic volume scattering for transect \#3 through the internal wave field along with VPR estimates of (top to bottom) large amphipod, large copepod, pteropod, salp, small amphipods, small copepods, unidentified gelatinous animals and unidentified animals. Animal abundance data were not collected after yearday 289.84 due to a missed tape change. 


\section{Appendix $\mathrm{C}$}

\section{Zooplankton Scattering Models}

The models used in the initial Forward Problem Calculations are those used by Stanton et al. (1994a) and Wiebe et al. (1996). They are presented here for convenience, with no substantial changes having been made.

\section{C.1 Variables}

$\beta_{D}$, ratio of length to width of the animal $\left(\beta_{D}=\frac{L}{D}\right)$

$R$, Reflection coefficient $\left(R=\frac{g h-1}{g h+1}\right)$

$g$, ratio of animal density to that of surrounding medium $\left(g=\frac{\rho_{\text {amimal }}}{\rho_{\text {water }}}\right)$

$h$, ratio of speed of sound in the animal to sound speed in the surrounding medium

$\left(h=\frac{c_{\text {animal }}}{c_{\text {water }}}\right)$

$k$, acoustic wavenumber $\left(k=\frac{2 \pi f}{c}=\frac{2 \pi}{\lambda}\right)$

$c$, speed of sound (in sea water, $c \sim 1500 \mathrm{~m} / \mathrm{s}$ )

$\lambda$, acoustic wavelength $\left(\lambda=\frac{f}{c}\right)$

$f$, acoustic frequency (in Hertz)

$T S$, Target Strength (dB)

RTS, Reduced Target Strength (dB)

$s_{v}$, volume scattering coefficient $\left(s_{v}=n \sigma_{b s}\right)$

$S_{v}$, volume scattering strength $\left(S_{v}=10 \log \frac{s_{v}}{s_{v, r e f}}\right)$ 
$n$, number of animals in the scattering region $\left(\# / \mathrm{m}^{3}\right)$

$\sigma_{b s}$, back-scattering cross-section

$s$, relative standard deviation of length (typically $s=0$ in our calculations)

$D$, width or cylindrical diameter

$a$, radius of animal or equivalent spherical radius

$L$, total length of animal (for fluid-like animals, this is normally measured from the eye to the telson)

$d_{\text {ratio }}$, body diameter ratio used to convert lengths of animals to $D$

\section{C.2 Fluid-like Animals}

Scattering from fluid-like animals is based on the bent cylinder model of (Stanton et al., 1993b, 1994a). It is an average over a uniform distribution of animal orientations. This model is used for all elongated fluid-like animals which includes: euphausiids, decapod shrimp, amphipods, chaetognaths, larval crustaceans, polychaetes, ostracods and cyphaunautes.

$$
\left\langle\sigma_{b s}\right\rangle=\frac{0.08 R^{2} L^{2}}{\beta_{D}}\left[1-e^{\left(\frac{-8 \pi^{2} f^{2} D^{2} s^{2}}{c^{2}}\right)} \cos \left(\frac{\pi f D}{c}\left(4-\frac{p i}{2\left(\frac{p i f D}{c}+0.4\right)}\right)\right)\right]
$$

where $\beta_{D}=2.5497, R=0.058$, and $d_{\text {ratio }}=0.3922$ for most animals. Exceptions include: $\beta_{D}=5.3576$ and $d_{\text {ratio }}=0.18665$ for euphausiids; $\beta_{D}=3.0021$ and $d_{\text {ratio }}=0.33315$ for amphipods; $\beta_{D}=17.151$ and $d_{\text {ratio }}=0.0583$ for chaetognaths and polychaetes.

\section{C.3 Copepods}

The scattering from copepods is found by using a "look-up" table (Figure C-1) which contains the Reduced Target Strength (RTS) for various values of ka. The look-up 
table is based on a fluid-like scattering function with the following parameters: $\beta_{D}=$ $2.5497, R=0.058$.

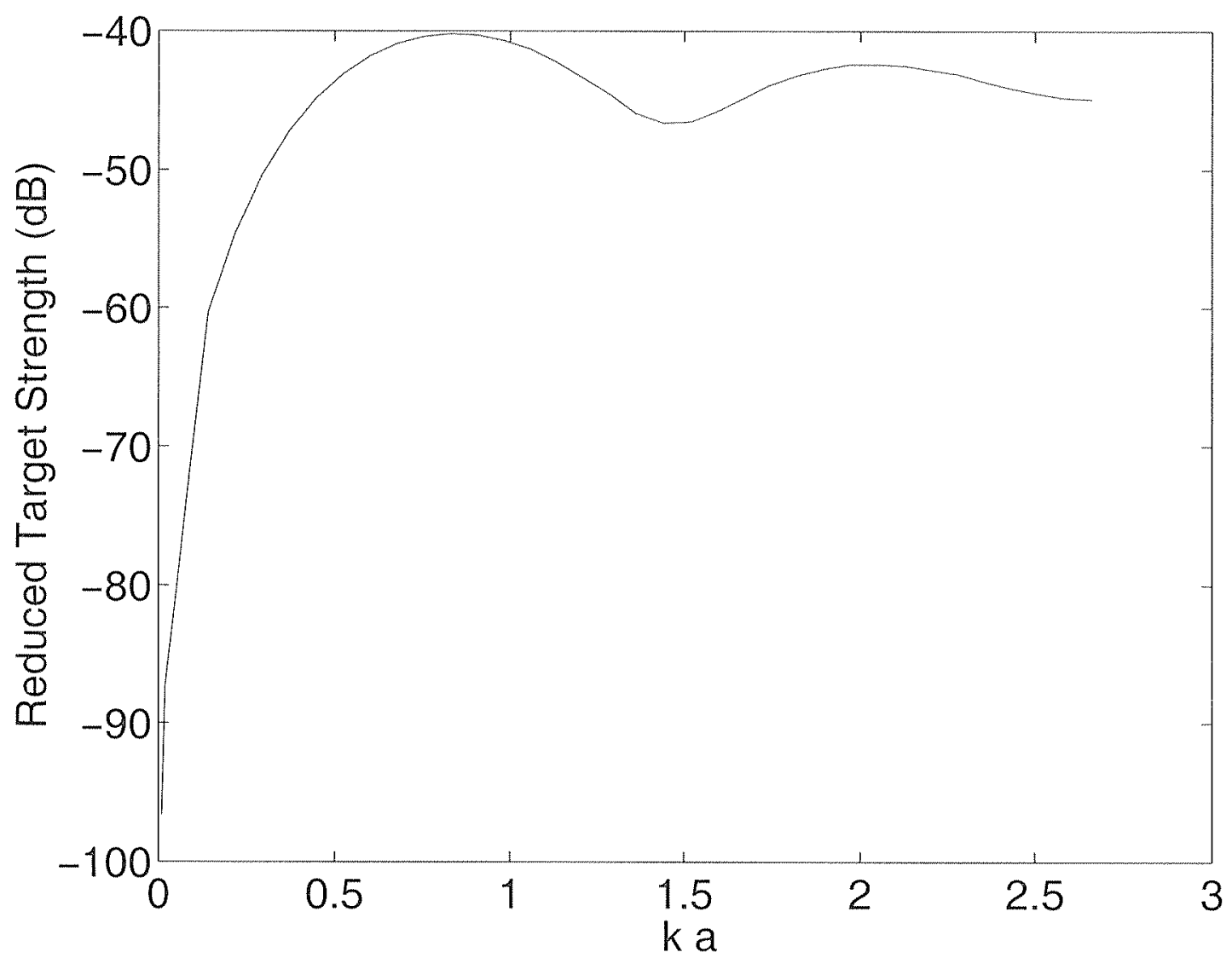

Figure C-1: Scattering spectra for a fluid-like animal (copepod) used in the Forward Problem calculations

The RTS is then converted to a Target Strength by the formula

$$
T S=R T S+10 \log \left(L^{2}\right)
$$

The TS values can then be converted into the volume backscattering coefficient by

$$
s_{v}=10^{\frac{T S}{10}}
$$

The $s_{v}$ values are used when summing up the contributions of the different animals and taxanomic types to the total volume scattering coefficient of a particular region. 


\section{C.4 Elastic-shelled Animals}

Scattering from elastic-shelled animals (pteropods and eggs in our samples) uses a dense fluid-sphere "high-pass" model

$$
\left\langle\sigma_{b s}\right\rangle=\frac{25 \pi^{4} D^{6} f^{4} R^{2}}{144 c t\left(1+\frac{\frac{25}{9} \pi^{4} f^{4} D^{4}}{c^{4}}\right)}
$$

For Limacina pteropods $R=0.5$, and for eggs $R=0.058$.

\section{C.5 Gelatinous Animals}

Scattering from gelatinous animals (medusae, salps) and the gelatinous tissue of siphonophores (bractophores and nectophores) is modeled using a simple back-scattering cross-section formula with the appropriate reflection coefficient

$$
\left\langle\sigma_{b s}\right\rangle=\frac{r_{1}^{2}+r_{2}^{2}}{4} R^{2}
$$

where $r_{1}, r_{2}$ are the major and minor radii of the cross-sectional area of the scatterer (many gelatinous animals have an elliptical rather than spherical or cylindrical shape thus they are modeled as ellipses). $R=0.028$ for all gelatinous animals modeled, however the radii values are different for medusa $\left(r_{1}-0.75, r_{2}=2.1\right)$ and salps, siphonophore nectophores, and bractophores $\left(r_{1}=.25, r_{2}=.75\right)$.

\section{C.6 Siphonophore Pneumatophore}

The gas-bearing pneumatophore of the siphonophore is a very strong scatterer (due to the density and sound speed contrasts between the carbon monoxide gas and the surrounding sea water). It is modeled using a look-up table (Figure C-2) that is based upon the scattering model for a fluid sphere developed by Anderson (1950). Stanton et al. (1998a) showed that most of the scattered energy from a siphonophore was from the gas inclusion. 


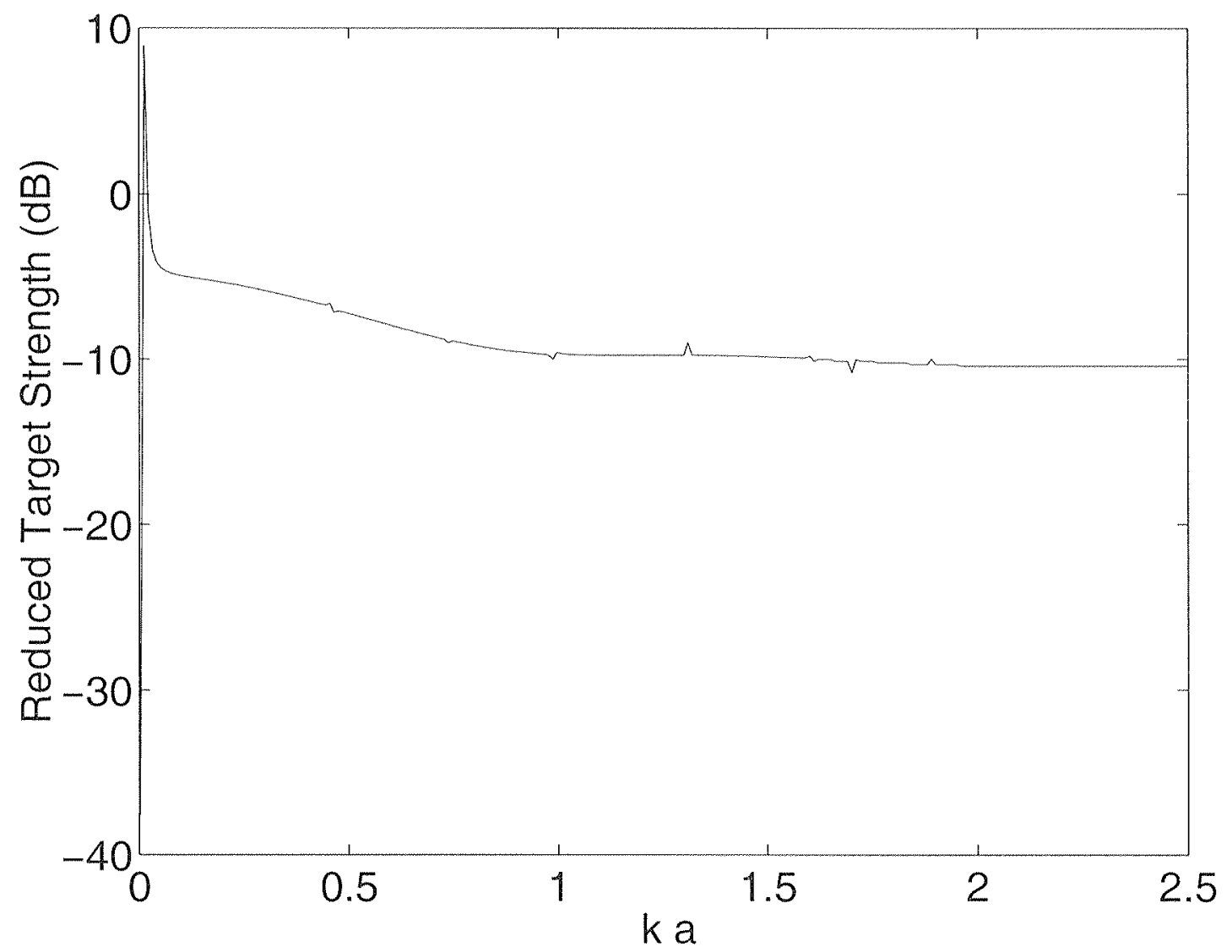

Figure C-2: Scattering spectra for the pneumatophore of a siphonophore modeled as a gas bubble. The RTS peak at a low value of ka is due to the resonance of the bubble. 


\section{Bibliography}

Allen, S. (1998). "A top N.E. fishing ground collapses, cod stocks drop in Gulf of Maine," Boston Globe, Dec 4.

Allen, S. (1999). "Fishermen bridle as restrictions get tougher, supply grows; New curbs eyed for Georges Bank," Boston Globe, Aug 30.

Anderson, V. C. (1950). "Sound scattering from a fluid sphere," Journal of the Acoustical Society of America 22, 426-431.

Backus, R. H. Editor (1987). Georges Bank, (MIT Press, Cambridge).

Baggeroer, A. B., Sperry, B., Lashkari, K., Chiu, C. S., Miller, J. H., Mikhalevsky, P. N., and von Der Heydt, K. (1994). "Vertical array receptions of the Heard Island transmissions," Journal of the Acoustical Society of America 96, 2395-2413.

Barham, E. G. (1963). "Siphonophores and the deep scattering layer," Science 140, $826-828$.

Barham, E. G. (1966). "Deep scattering layer migration and composition: Observations from a diving saucer." Science 151, 1399-1403.

Bary, B. M. (1966). "Back scattering at $12 \mathrm{kc} / \mathrm{s}$ in relation to biomass and number of zooplankton organisms in Saanich Inlet, British Columbia," Deep-Sea Research. I $13,655-666$.

Bary, B. M., and Pieper, R. E. (1971). "Sonic-scattering studies in Saanich Inlet, British Columbia: A Preliminary report." (Dept. of the Navy, Maury Center for Ocean Science, Report No. MC-005).

Batchelor, G. K. (1959). "Small-scale variation of convected quantities like temperature in turbulent fluid. Part I. General discussion and the case of small conductivity," Journal of Fluid Mechanics 5, 113-133.

Beamish, P. (1971). "Quantitative measurements of acoustic scattering from zooplanktonic organisms," Deep-Sea Research I 18, 811-822. 
Benfield, M. C., Davis, C. S., Wiebe, P. H., Gallager, S. M., Lough, R. G., and Copley, N. J. (1996). "Video Plankton Recorder estimates of copepod, pteropod and larvacean distributions from a stratified region of Georges Bank with comparative measurements from a MOCNESS sampler," Deep-Sea Research II 43, 1925-1945.

Benfield, M. C., Davis, C. S., and Gallager, S. M. (2000). "Estimating the in-situ orientation of Calanus finmarchicus on Georges Bank using the Video Plankton Recorder," Plankton Biology and Ecology 47, 69-72.

Bigelow, H. B. (1926). Plankton of the offshore waters of the Gulf of Maine (Washington: United States Government Printing Office).

Bigelow, H. B. (1927). Physical oceanography of the Gulf of Maine (Washington: United States Government Printing Office).

Bigelow, H. B., and Schroeder, W. C. (1953). Fishes of the Gulf of Maine (Washington: United States Government Printing Office).

Biggs, D. C. (1977). "Field studies of fishing, feeding and digestion in Siphonophores," Marine Behaviour and Physiology 4, 261-274.

Brierley, A. S., Brandon, M. A., and Watkins, J. L. (1998). "An assessment of the utility of an acoustic Doppler current profiler for biomass estimation," Deep-Sea Research I 45, 1555-1573.

Butman, B., and Beardsley, R. C. (1987). Physical Oceanography in Georges Bank, edited by R. H. Backus, (MIT Press), pp. 88-98.

Castile, B. D. (1975). "Reverberation from plankton at $330 \mathrm{kHz}$ in the western $\mathrm{Pa}$ cific," Journal of the Acoustical Society of America 58, 972-976.

Caswell, H., Fujiwara, M., and Brault, S. (1999). "Declining survival probability threatens the North Atlantic right whale," Proceedings of the National Academy of Sciences of the United States of America 96, 3308 3313.

Chamberlain, T. (1985). "Will success ruin N.E. fish industry? Some species are becoming casualties of a sophisticated technical revolution," Boston Globe , May 27.

Chu, D., Stanton, T. K., and Wiebe, P. H. (1992). "Frequency dependence of sound backscattering from live individual zooplankton," ICES Journal of Marine Science 49, 97-106.

Chu, D., Foote, K. G., and Stanton, T. K. (1993). "Further analysis of target strength measurements of Antarctic krill at 38 and $120 \mathrm{kHz}$ : Comparison with deformed cylinder model and inference of orientation distribution," Journal of the Acoustical Society of America 93, 2985-2988. 
Chu, D., and Stanton, T. K. (1998). "Application of pulse compression techniques to broadband acoustic scattering by live individual zooplankton," Journal of the Acoustical Society of America 104, 39-55.

Chu, D., Wiebe, P. H., and Copley, N. (2000a). "Inference of material properties of zooplankton from acoustic and resistivity measurements," ICES Journal of Marine Science 57, 1128-1142.

Chu, D., Wiebe, P. H., Stanton, T. K., Hammar, T. R., Doherty, K. W., Copley, N. J., Zhang, J., Reeder, D. B., and Benfield, M.C. (2000b). "Measurements of the material properties of live marine organisms," Proc of the OCEANS 2000 MTS/IEEE International Symposium. Providence, RI. September 11-14, Vol. 3, pp $1963-1967$.

Clay, C. S. (1983). "Deconvolution of the fish scattering PDF from the echo PDF for a single transducer sonar." Journal of the Acoustical Society of America 73, $1989-1994$.

Croll, D. A., and Tershy, B. R. (1998). "Penguins, fur seals, and fishing: prey requirements and potential competition in the South Shetland Islands, Antarctica," Polar Biology 19, 365-374.

David, P. M., Guerin-Ancey, O., and Cuyck, J. P. V. (1999). "Acoustic discrimination of two zooplankton species (mysid) at 38 and $120 \mathrm{kHz}$," Deep-Sea Research I 46, 319-333.

Davis, C. S. (1987). Zooplankton Life Cycles in Georges Bank edited by R. H. Backus, (MIT Press), pp. 256-267.

Davis, C. S., and Wiebe, P. H. (1985). "Macrozooplankton biomass in a warm-core Gulf Stream ring: Time series changes in size, structure, and taxonomic composition and vertical distribution," Journal of Geophysical Research 90, 8871-8884.

Davis, C. S., Gallager, S. M., Berman, M. S., Haury, L. R., and Strickler, J. R. (1992). "The Video Plankton Recorder (VPR): Design and initial results," Archiv für Hydrobiologie 36, 67-81.

Dietz, R. S. (1948). "Deep scattering layer in the Pacific and Antarctic oceans," Journal of Marine Research 7, 430-442.

Dillon, T. M. (1982). "Vertical overturns: A comparison of Thorpe and Ozmidov length scales," Journal of Geophysical Research 87, 9601-9613.

Dumanoski, D. (1988). "Codfish population on Georges Bank in peril, study says," Boston Globe, Nov 5.

Editorial (1995). "Tough measures on fishing backed," Boston Globe, Oct 26. 
Foote, K. G. (1990). "Speed of sound in Euphausia superba," Journal of the Acoustical Society of America 87, 1405-1408.

Foote, K. G., and Stanton, T. K. (2000). Acoustical Methods (Ch. 6) in ICES Zooplankton Methodology Manual edited by R. Harris, P. H. Wiebe, J. Lenz, H. R. Skjoldal, and M. Huntley, (Academic Press), pp. 223-258.

Gilmer, R. W., and Harbison, G. R. (1986). "Morphology and field behavior of pteropod molluscs: feeding methods in the families Cavoliniidae, Limacinidae and Peraclididae (Gastropoda: Thecosomata)," Marine Biology 91, 47-57.

Goodman, L. (1990). "Acoustic scattering from ocean microstructure," Journal of Geophysical Research 95, 11557-11573.

Gould, S. J. (1984). "A Most Ingenious Paradox," Natural History 12, 20-30.

Greene, C. H., Wiebe, P. H., Pershing, A. J., Gal, G., Popp, J. M., Copley, N. J., Austin, T. C., Bradley, A. M., Goldsborough, R. G., Dawson, J., Hendershott, R., and Kaartvedt, S. (1998). "Assessing the distribution and abundance of zooplankton: a comparison of acoustic and net-sampling methods with D-BAD MOCNESS," Deep-Sea Research II 45, 1219-1237.

Greenlaw, C. F. (1977). "Backscattering spectra of preserved zooplankton," Journal of the Acoustical Society of America 62, 44-52.

Greenlaw, C. F. (1979). "Acoustical estimation of zooplankton populations," Limnology and Oceanography 24, 226-242.

Gregg, M. (1987). "Diapycnal mixing in the thermocline: A review," Journal of Geophysical Research 92, 5249-5286.

Hansen, W. J., and Dunbar, M. J. (1971). "Biological causes of scattering layers in the Arctic Ocean," (Dept. of the Navy, Maury Center for Ocean Science, Report No. MC-005).

Haury, L., Wiebe, P., Orr, W., and Briscoe, M. (1983). "Tidally generated highfrequency internal wave packets and their effects on plankton in Massachusetts Bay," Journal of Marine Research 41, 65-112.

Hebert, D., Moum, J. N., Paulson, C. A., and Caldwell, D. R. (1992). "Turbulence and internal waves at the Equator. Part II: Details of a single event," Journal of Physical Oceanography 22, 1346-1356.

Hersey, J. B., and Backus, R. H. (1954). "New evidence that migrating gas bubbles, probably the swimbladders of fish, are largely responsible for scattering layers on the continental rise south of New England," Deep-Sea Research I 1, 190-191. 
Holliday, D. V. (1977). Extracting bio-physical information from the acoustic signatures of marine organisms in Oceanic Sound Scattering Prediction edited by N. R. Andersen and B. J. Zahuranec, (Plenum Press), pp. 619-624.

Holliday, D. V., and Pieper, R. E. (1995). "Bioacoustical oceanography at high frequencies," ICES Journal of Marine Science 52, 279-296.

Holliday, D. V., Pieper, R. E., and Kleppel, G. S. (1989). "Determination of zooplankton size and distribution with multifrequency acoustic technology," ICES Journal of Marine Science 46, 52-61.

Howe, P. J. (1999). "Widespread ban on ground fishing offered to save cod," Boston Globe, Jan 30.

Johnson, M. W. (1948). "Sound as a tool in marine ecology, from data on biological noises and the deep scattering layer," Journal of Marine Research 7, 443-458.

Junger, M. C., and Feit, D. (1993). Sound, Structures, and Their Interaction (Acoustical Society of America).

Kargl, S. G., and Marston, P. L. (1989). "Observations and modeling of the backscattering of short tone bursts from a spherical shell: Lamb wave echoes, glory, and axial reverberations," Journal of the Acoustical Society of America 85, 1014-1028.

Kils, U. (1981). "The swimming behavior, swimming performance and energy balance of Antarctic krill, Euphausia superba," BIOMASS Science Series Vol. 3 .

Laidler, J. (1999). "State commission receives funding, tackles problems of fishing industry," Boston Globe, Jan 24.

Lennert-Cody, C. E., and Franks, P. J. S. (1999). "Plankton patchiness in highfrequency internal waves," Marine Ecology Progress Series 186, 59-66.

Mackie, G. O., Pugh, P. R., and Purcell, J. E. (1987). "Siphonophore biology," Advances in Marine Biology 24, 97-262.

Madin, L. P. (1988). "Feeding behavior of tentaculate predators: in situ observations and a conceptual model," Bulletin of Marine Science 43, 413-429.

Marshall, N. B. (1951). "Bathypelagic fishes as sound scatterers in the ocean," Journal of Marine Research 10, 1-17.

Martin, L. V., Stanton, T. K., Wiebe, P. H., and Lynch, J. F. (1996). "Acoustic classification of zooplankton," ICES Journal of Marine Science 53, 217-224.

Martin-Traykovski, L. V. (1998). Acoustic Classification of Zooplankton, Ph.D. thesis, Massachusetts Institute of Technology and Woods Hole Oceanographic Institution. 
McGehee, D. E., O'Driscoll, R. L., and Martin-Traykovski, L. V. (1998). "Effects of orientation on acoustic scattering from Antarctic krill at $120 \mathrm{kHz}$," Deep-Sea Research II 45, 1273-1294.

Medwin, H., and Clay, C. S. (1998). Fundamentals of Acoustical Oceanography (Boston: Academic Press).

Miyashita, K., and Aoki, I. (1999). "Acoustic measurements of zooplankton using a dual frequency echo sounder," Marine Ecology Progress Series 180, 105-109.

Miyashita, K., Aoki, I., and Inagaki, T. (1996). "Swimming behaviour and target strength of isada krill (Euphausia pacifica)," ICES Journal of Marine Science 53, 303-308.

Morton, J. E. (1954). "The biology of Limacina retroversa," Journal of the Marine Biological Association of the United Kingdom 33, $297-312$.

Napp, J. M., Ortner, P. B., Pieper, R. E., and Holliday, D. V. (1993). "Biovolume-size spectra of epipelagic zooplankton using a multi-frequency acoustic profiling system (MAPS)," Deep-Sea Research I 40, 445-459.

Pickwell, G. V., Barham, E. G., and Wilton, J. W. (1964). "Carbon monoxide production by a bathypelagic siphonophore," Science 144, 860-862.

Pieper, R. E., Holliday, D. V., and Kleppel, G. S. (1990). "Quantitative zooplankton distributions from multifrequency acoustics," Journal of Plankton Research 12 , $433-441$.

Pugh, P. R. (1975). "The distribution of siphonophores in a transect across the North Atlantic ocean at $32^{\circ} \mathrm{N}$," Journal of Experimental Marine Biology and Ecology 20, $77-97$.

Reeve, M. R. (1981). "Large cod-end reservoirs as an aid to the live collection of delicate zooplankton," Limnology and Oceanography 26, 577-579.

Rehmann, C. R., and Duda, T. F. (2000). "Diapycnal diffusivity inferred from scalar microstructure measurements near the New England shelf/slope front," Journal of Physical Oceanography 30, 1354-1371.

Robison, B. H., Reisenbichler, K. R., Sherlock, R. E., Silguero, J. M. B., and Chavez, F. P. (1998). "Seasonal abundance of the siphonophore, Nanomia bijuga, in Monterey Bay," Deep-Sea Research II 45, 1741-1751.

Rogers, C. A., Biggs, D. C., and Cooper, R. A. (1978). "Aggregation of the siphonophore Nanomia cara in the Gulf of Maine: Observations from a submersible," Fishery Bulletin 76, 281-284. 
Sameoto, D. D. (1980). "Quantitative measurements of Euphausiids using a 120-kHz sounder and their in situ orientation," Canadian Journal of Fisheries and Aquatic Science 37, 693-702.

Sandstrom, H., Elliott, J. A., and Cochrane, N. A. (1989). "Observing groups of solitary internal waves and turbulence with BATFISH and echo-sounder," Journal of Physical Oceanography 19, 987-997.

Seim, H. E. (1999). "Acoustic backscatter from salinity microstructure," Journal of Atmospheric and Oceanic Technology 16, 1491-1498.

Seim, H. E., and Gregg, M. C. (1994). "Detailed observations of a naturally occurring shear instability," Journal of Geophysical Research 99, 10,049-10,073.

Seim, H. E., Gregg, M. C., and Miyamoto, R. T. (1995). "Acoustic backscatter from turbulent microstructure," Journal of Atmospheric and Oceanic Technology 12, $367-380$.

Smith, P. F. (1954). "Further measurements of the sound scattering properties of several marine organisms," Deep-Sea Research I 2, 71-79.

Stanton, T. K. (1990). "Sound scattering by spherical and elongated shelled bodies," Journal of the Acoustical Society of America 88, 1619-1633.

Stanton, T. K., and Clay, C. S. (1986). "Sonar echo statistics as a remote-sensing tool: Volume and seafloor," IEEE Joumal of Oceanic Engineering 11(1), 79-96.

Stanton, T. K., Clay, C. S., and Chu, D. (1993a). "Ray representation of sound scattering by weakly scattering deformed fluid cylinders: Simple physics and application to zooplankton," Journal of the Acoustical Society of America 94, 3454-3462.

Stanton, T. K., Chu, D., Wiebe, P. H., and Clay, C. S. (1993b). "Average echoes from randomly oriented random-length finite cylinders: Zooplankton models," Journal of the Acoustical Society of America 94, 3463-3472.

Stanton, T. K., Wiebe, P. H., Chu, D., Benfield, M. C., Scanlon, L., Martin, L., and Eastwood, R. L. (1994a). "On acoustic estimates of zooplankton biomass," ICES Journal of Marine Science 51, 505-512.

Stanton, T. K., Wiebe, P. H., Chu, D., and Goodman, L. (1994b). "Acoustic characterization and discrimination of marine zooplankton and turbulence," ICES Journal of Marine Science 51, 469-479.

Stanton, T. K., Chu, D., and Wiebe, P. H. (1996). "Acoustic scattering characteristics of several zooplankton groups," ICES Journal of Marine Science 53, 289-295. 
Stanton, T. K., Chu, D., Wiebe, P. H., Martin, L. V., and Eastwood, R. L. (1998a). "Sound scattering by several zooplankton groups. I. Experimental determination of dominant scattering mechanisms," Journal of the Acoustical Society of America $103,225-235$.

Stanton, T. K., Chu, D., and Wiebe, P. H. (1998b). "Sound scattering by several zooplankton groups. II. Scattering models." Journal of the Acoustical Society of America 103, 236-253.

Stanton, T. K., Wiebe, P. H., and Chu, D. (1998c). "Differences between sound scattering by weakly scattering spheres and finite-length cylinders with applications to sound scattering by zooplankton," Journal of the Acoustical Society of America $103,254-264$.

Stanton, T. K., Chu, D., Wiebe, P. H., Eastwood, R. L., and Warren, J. D. (2000a). "Acoustic scattering by benthic and pelagic shelled animals," Journal of the Acoustical Society of America 108, 535-550.

Stanton, T. K., and Chu, D. (2000b). "Review and recommendations for the modelling of acoustic scattering by fluid-like elongated zooplankton: euphausiids and copepods," ICES Journal of Marine Science 57, 793-807.

Stepnowski, A., and Moszynski, M. (2000). "Inverse problem solution techniques as applied to indirect in situ estimation of fish target strength," Journal of the Acoustical Society of America 107, 2554-2562.

Terry, S. M. (1994). "Georges Bank proposal worries fisherman, federal officials consider closure to replenish supply of ground fish," Boston Globe, Aug 21.

Thorpe, S. A. (1987). "Transitional phenomena and the development of turbulence in stratified fluids: A review," Journal of Geophysical Research 92, 5231-5248.

Totton, A. K. (1965). A Synopsis of the Siphonophora (British Museum (Natural History)).

Trevorrow, M. V. (1998). "Observations of internal solitary waves near the Oregon coast with an inverted echo sounder," Journal of Geophysical Research 103, 76717680 .

Trevorrow, M. V., and Teichrob, R. C. (1994). "Self-contained acoustics platforms for probing ocean surface processes," IEEE Journal of Oceanic Engineering 19, $483-492$.

Turin, G. L. (1960). "An introduction to matched filters," IRE Transactions on Information Theory IT-6, 311-329.

Urick, R. J. (1983). Principles of Underwater Sound (Peninsula Publishing). 
Wiebe, P. H. (1988). "Functional regression equations for zooplankton displacement volume, wet weight, dry weight, and carbon: A correction," Fishery Bulletin 86, $833-835$.

Wiebe, P. H., Boyd, S. H., and Cox, J. (1975). "Relationships between zooplankton displacement volume, wet weight, dry weight, and carbon," Fishery Bulletin 73, $777-786$.

Wiebe, P. H., Morton, A. W., Bradley, A. M., Backus, R. H., Craddock, J. E., Barber, V., Cowles, T. J., and Flierl, G. R. (1985). "New developments in the MOCNESS, an apparatus for sampling zooplankton and micronekton," Marine Biology 87, 313 323.

Wiebe, P. H., Mountain, D. G., Stanton, T. K., Greene, C. H., Lough, G., Kaartvedt, S., Dawson, J., and Copley, N. (1996). "Acoustical study of the spatial distribution of plankton on Georges Bank and the relationship between volume backscattering strength and the taxonomic composition of the plankton," Deep-Sea Research II 43, $1971-2001$.

Wiebe, P. H., Stanton, T. K., Benfield, M. C., Mountain, D. G., and Greene, C. H. (1997). "High-frequency acoustic volume backscattering in the Georges Bank coastal region and its interpretation using scattering models," IEEE Journal of Oceanic Engineering 22, 445-464.

Wiebe, P. H., Stanton, T. K., Greene, C. H., Benfield, M. C., Sosik, H. M., Austin, T., Warren, J. D., and Hammer, T. (submitted). "BIOMAPER II: An integrated instrument platform for coupled biological and physical measurements in coastal and oceanic regimes," IEEE Journal of Oceanic Engineering .

Woods, J. D. (1977). Turbulence as a factor in sound scattering in the upper ocean in Oceanic Sound Scattering Prediction edited by N. R. Andersen and B. J. Zahuranec (Plenum Press), pp. 129-145.

Ye, Z. (1997). "A novel approach to sound scattering by cylinders of finite length," Journal of the Acoustical Society of America 102, 877-884.

Zhang, L. G., Sun, N. H., and Marston, P. L. (1992). "Midfrequency enhancement of the backscattering of tone bursts by thin spherical shells," Journal of the Acoustical Society of America 91, 1862-1874.

Zitner, A. (1999). "Clinton to urge $\$ 48 \mathrm{M}$ plan to aid fishermen in region," Boston Globe, Jan 30. 